\title{
User's Guide for the NREL Teetering Rotor Analysis Program (STRAP)
}

Alan D. Wright

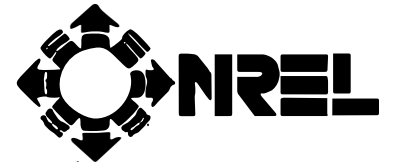

National Renewable Energy Laboratory

(formerly the Solar Energy Research Institute) 1617 Cole Boulevard

Golden, Colorado 80401-3393

A Division of Midwest Research Institute Operated for the U.S. Department of Energy under Contract No. DE-AC02-83CH10093 


\section{NOTICE}

This report was prepared as an account of work sponsored by an agency of the United States government. Neither the United States government nor any agency thereof, nor any of their employees, makes any warranty, express or implied, or assumes any legal liability or responsibility for the accuracy, completeness, or usefulness of any information, apparatus, product, or process disclosed, or represents that its use would not infringe privately owned rights. Reference herein to any specific commercial product, process, or service by trade name, trademark, manufacturer, or otherwise does not necessarily constitute or imply its endorsement, recommendation, or favoring by the United States government or any agency thereof. The views and opinions of authors expressed herein do not necessarily state or reflect those of the United States government or any agency thereof.

Printed in the United States of America

Available from:

National Technical Information Service

U.S. Department of Commerce

5285 Port Royal Road

Springfield, VA 22161

Price: Microfiche A01

Printed Copy A10

Codes are used for pricing all publications. The code is determined by the number of pages in the publication. Information pertaining to the pricing codes can be found in the current issue of the following publications which are generally available in most libraries: Energy Research Abstracts (ERA); Government Reports Announcements and Index (GRA and I); Scientific and Technical Abstract Reports (STAR); and publication NTIS-PR-360 available from NTIS at the above address. 


\section{Summary}

The following report gives the reader an overview of instructions on the proper use of the National Renewable Energy Laboratory (formerly the Solar Energy Research Institute, or $\underline{S} E R I)$ Teetering Rotor Analysis Program (STRAP version 2.20). STRAP is a derivative of the Force and Loads Analysis Program (FLAP). It is intended as a tool for prediction of rotor and blade loads and response for only two-bladed teetering hub wind turbines. The effects of delta-3, undersling, hub mass, and wind turbulence are accounted for.

The objectives of the report are to give an overview of the code and also show the methods of data input and correct code execution steps in order to model an example two-bladed teetering hub turbine. A large portion of the discussion (Sections 6.0, 7.0, and 8.0) is devoted to the subject of inputting and running the code for wind turbulence effects. The ability to include turbulent wind effects is perhaps the biggest change in the code since the release of FLAP version 2.01 in 1988.

This report is intended to be a user's guide. It does not contain a theoretical discussion on equations of motion, assumptions, underlying theory, etc. It is intended to be used in conjunction with Wright, Buhl, and Thresher (1988).

Hopefully, this report will assist new users in the understanding of code input preparation and correct code execution. 


\section{Table of Contents}

$\underline{\text { Page }}$

Summary $\ldots \ldots \ldots \ldots \ldots \ldots \ldots \ldots \ldots \ldots \ldots \ldots \ldots \ldots \ldots \ldots$

Nomenclature $\ldots \ldots \ldots \ldots \ldots \ldots \ldots \ldots \ldots \ldots \ldots \ldots \ldots \ldots \ldots$ vii

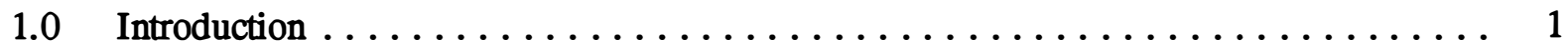

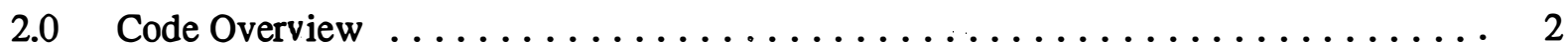

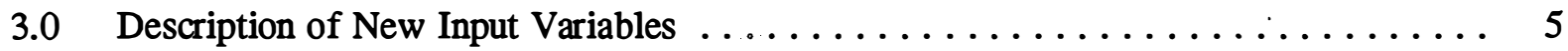

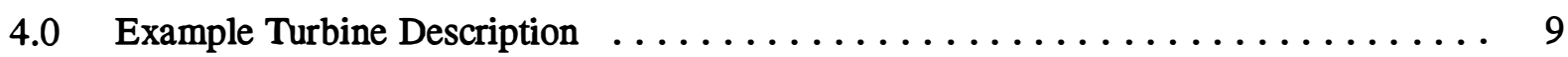

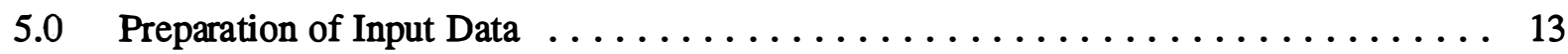

6.0 Preparation of Turbulent Wind Input Data $\ldots \ldots \ldots \ldots \ldots \ldots \ldots \ldots$

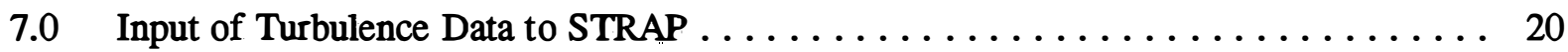

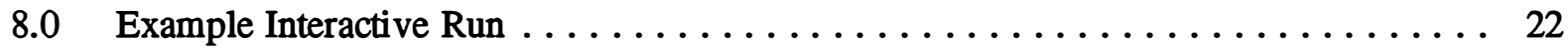

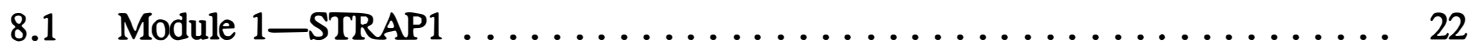

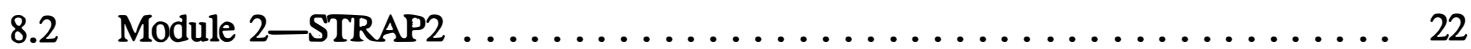

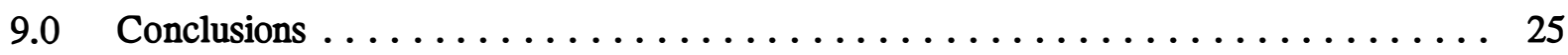

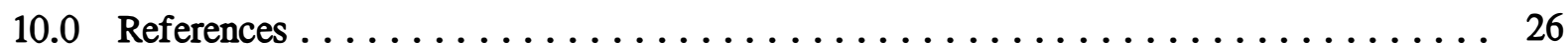

11.0 Acknowledgments $\ldots \ldots \ldots \ldots \ldots \ldots \ldots \ldots \ldots \ldots \ldots \ldots \ldots \ldots \ldots \ldots$

Appendix A: Module 1 Input File, Interactive Run Listing, and Output Files . . . . . . . A-1 Appendix B: Module 2 Interactive Run Listing and Output Files $\ldots \ldots \ldots \ldots \ldots \ldots$. . . . Appendix C: Module 1 Listing $\ldots \ldots \ldots \ldots \ldots \ldots \ldots \ldots \ldots \ldots \ldots \ldots \ldots \ldots \ldots \ldots \ldots \ldots$

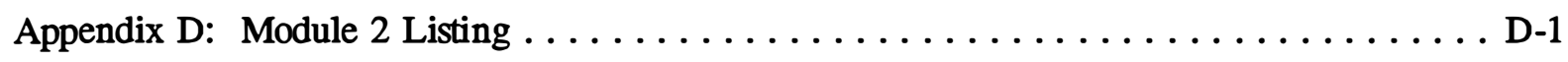

Appendix E: Modules 1 and 2 Flowcharts $\ldots \ldots \ldots \ldots \ldots \ldots \ldots \ldots \ldots \ldots \ldots \ldots$ 


\section{List of Figures}

$\underline{\text { Page }}$

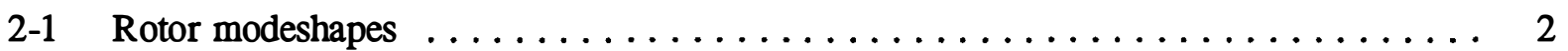

2-2 Illustration of the teetering rotor with $\delta-3$, undersling,

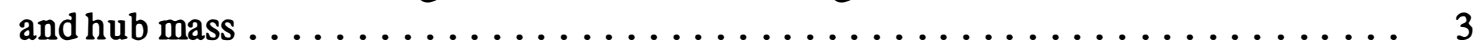

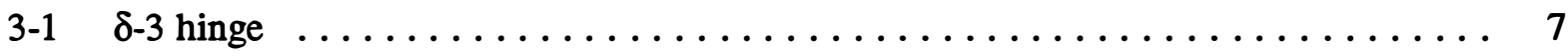

3-2 Illustration of the Teeter Springs and Damper Assembly $\ldots \ldots \ldots \ldots \ldots \ldots$

4-1 Illustration of example turbine $\ldots \ldots \ldots \ldots \ldots \ldots \ldots \ldots \ldots \ldots \ldots$

4-2 Turbine blade planform, thickness, and twist $\ldots \ldots \ldots \ldots \ldots \ldots \ldots \ldots \ldots \ldots \ldots \ldots$

4-3 Turbine blade stiffness distribution $\ldots \ldots \ldots \ldots \ldots \ldots \ldots \ldots \ldots \ldots \ldots \ldots \ldots \ldots \ldots \ldots$

4-4 Turbine blade weight distribution $\ldots \ldots \ldots \ldots \ldots \ldots \ldots \ldots \ldots \ldots \ldots \ldots \ldots$

4-5 Lift profile for the $L S(1)$ airfoil $\ldots \ldots \ldots \ldots \ldots \ldots \ldots \ldots \ldots \ldots \ldots \ldots \ldots$

5-1 Illustration of rotor geometry $\ldots \ldots \ldots \ldots \ldots \ldots \ldots \ldots \ldots \ldots \ldots \ldots$ 


\section{List of Tables}

$\underline{\text { Page }}$

3-1 New Input Variable Description $\ldots \ldots \ldots \ldots \ldots \ldots \ldots \ldots \ldots \ldots \ldots \ldots \ldots \ldots \ldots$

4-1 Example Turbine Specifications $\ldots \ldots \ldots \ldots \ldots \ldots \ldots \ldots \ldots \ldots \ldots \ldots \ldots \ldots$ 


\section{Nomenclature}

A

C

$\mathrm{C}_{\mathrm{D}} \quad$ Drag coefficient

$\mathrm{C}_{\mathrm{L}} \quad$ Lift coefficient

deg Degrees

$\mathrm{ft} \quad$ Feet

m Meters

$\mathbf{M}_{\text {hub }}$

d

$\mathbf{u}$

$\mathrm{K}_{0} \quad$ Teeter hinge stiffness ( $\left.\mathrm{ft}-\mathrm{lb} / \mathrm{rad}\right)$

$\mathrm{K}_{1} \quad$ Stiffness of first teeter stop (ft-lb/rad) (see Figure 3-2)

$\mathrm{K}_{2} \quad$ Stiffness of second teeter hard-stop (ft-lb/rad) (see Figure 3-2)

rad Radians

$B_{1} \quad$ Angle of teeter at which blade hits first teeter stop (deg) (see Figure 3-2)

$B_{2} \quad$ Angle of teeter at which blade hits second hard stop (deg) (see Figure 3-2)

6-3 delta-3 hinge angle (see Figure 3-1) (deg)

$\Omega \quad$ Rotor rotation rate (RPM) 


\subsection{Introduction}

The following report is intended to be a guide for the correct use of the National Renewable Energy Laboratory (NREL) (formerly $\underline{S E R I)}$ Teetering Rotor Analysis Program (STRAP). STRAP is a derivative of the Force and Loads Analysis Program (FLAP). The original equations of motion that formed the basis for FLAP (version 2.01) have been reformulated to correctly predict loads and response for two-bladed teetering hub rotors.

The code accounts for such effects as delta-3, undersling, hub mass, and wind turbulence. Degrees of freedom include rotor teeter and three-blade elastic flap modes. A prescribed time-dependent sinusoidal yaw function can be input to the code.

The report gives a general overview of the code. A description of new input variables (compared to FLAP version 2.01 of 1988) is given. Then, an example of a two-bladed teetering hub wind turbine is described. Preparation of the basic data input file for this two-bladed turbine is described. Then, the methods of turbulent wind input preparation and input of turbulence to STRAP are explained. Finally, an interactive

run session for both modules is illustrated. Code listings and output results are contained in the appendices. 


\subsection{Code Overview}

STRAP analyzes two-bladed teetering hub rotors. It predicts blade and low-speed shaft loads that result from such effects as gravity, windshear, tower shadow, and turbulent wind fluctuations.

Degrees of freedom included in this model are rotor teeter and three elastic flap modes, as seen in Figure 2-1. Blade torsion and edgewise degrees of freedom are not included. The tower top is assumed to be fixed in space. The model assumes constant rotor speed. Although machine yaw is not considered to be a degree of freedom, a prescribed time-dependent sinusoidal yaw motion can be input to the code.

STRAP is basically a derivative of FLAP (Wright, Buhl, and Thresher, 1988). Equations of motion have been reformulated to model the type of rotor shown in Figure 2-2. This rotor consists of two blades coupled together by a teetering hinge (at point A). The hinge may be skewed at an angle, $\delta-3$. The teetering hinge may also be located a distance $u(f t)$ downwind of the blades' apex point (0).

The total rotor mass may consists of two parts: (1) that part modeled as distributed mass of each blade and (2) that part due to the hub, not included as blade distributed mass. A description of blade mass input will be given in Section 3.0 on code input. In order to model the second part, the user can input a concentrated hub mass $\left(\mathrm{M}_{\mathrm{hub}}-\mathrm{lb}-\mathrm{s}^{2} / \mathrm{ft}\right)$ located a distance $\mathrm{d}(\mathrm{ft})$ downwind of point 0 . This point locates the hub center of gravity location (just the hub, with the two blades removed at the root). The blade tip masses shown in Figure 2-2 are modeled in STRAP by adding extra distributed blade mass to the last $2 \mathrm{ft}$ of blade span. Provisions for blade concentrated point masses are not included in STRAP.

The model also includes the effects of teeter springs and dampers. Teeter stop impacts can be modeled by appropriate choice of the spring stiffnesses, as will be described in Section 3.0.

This version of STRAP is similar to FLAP in that a quasisteady linear aerodynamic model is used to compute blade aerodynamic forces. The lift is modeled as a linear function of angle of attack up to stall. Past stall, lift is set equal to a constant. The drag is modeled as a quadratic function involving lift coefficient. For more details, see Section 5.0 or Wright, Buhl, and Thresher (1988).

2nd Asymmetric Mode

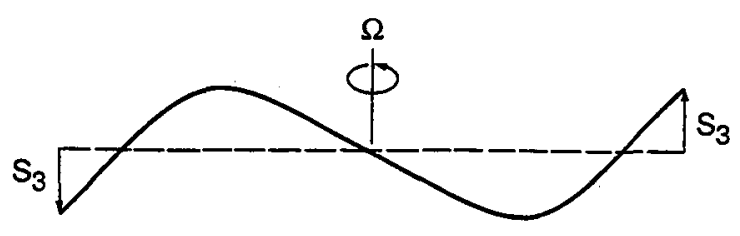

2nd Symmetric Mode

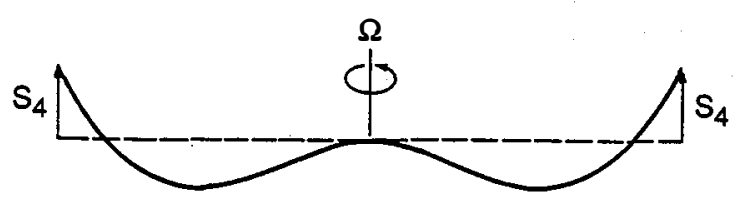

1st Asymmetric Mode (teeter)

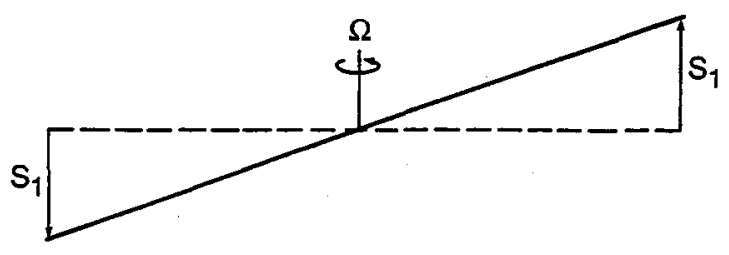

1st Symmetric Bending Mode

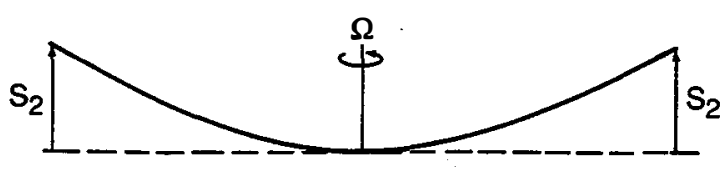

BACO245102

Figure 2-1. Rotor modeshapes 


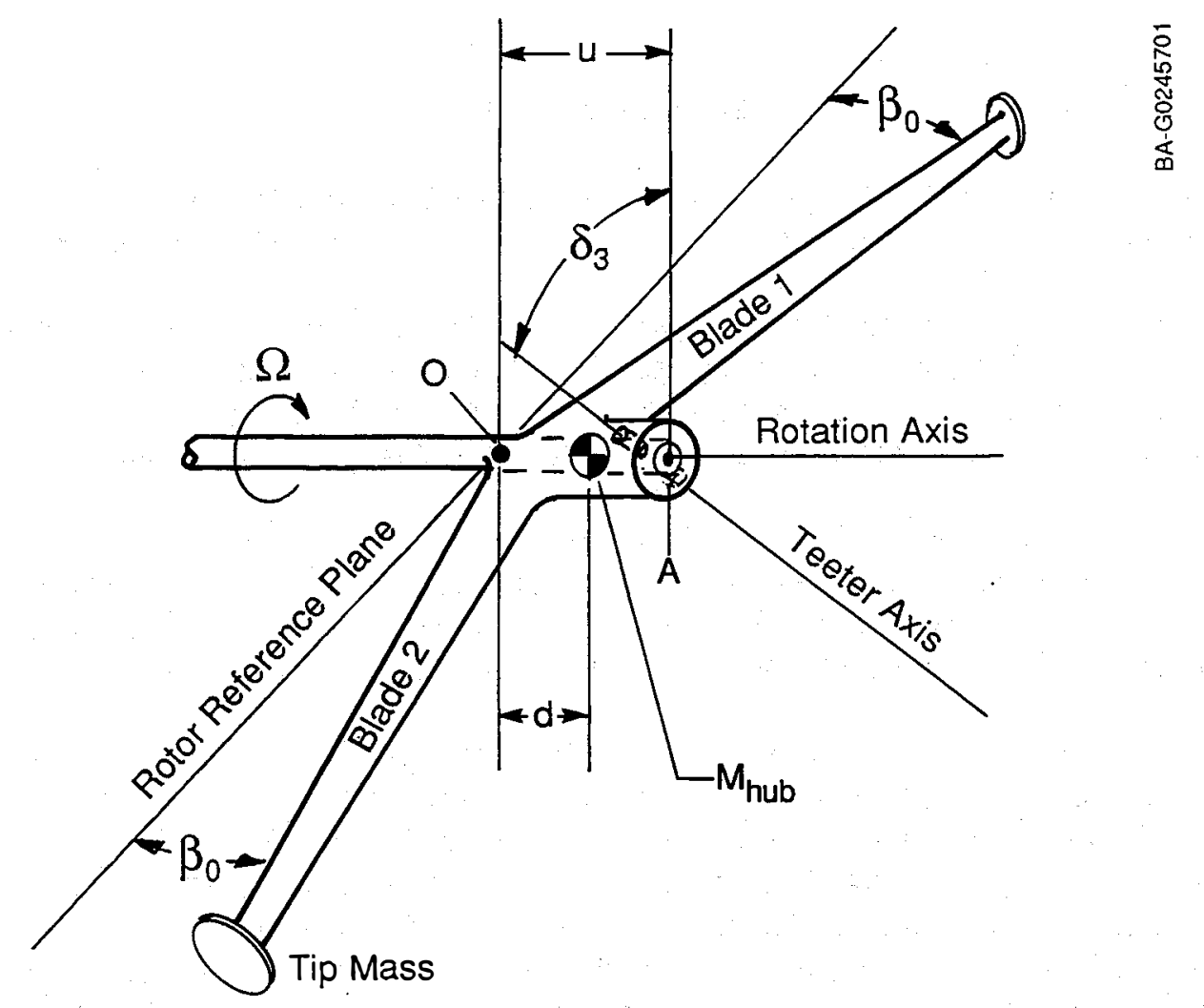

Figure 2-2. Illustration of the teetering rotor with $\delta-3$, undersling, and hub mass

STRAP is composed of two modules (STRAP1 and STRAP2). The first module is a preprocessor that reads blade and machine property data. It computes such items as blade flapwise frequencies and modeshapes as well as stiffness, mass, coriolis, and other matrices. These quantities are variables that do not generally change from one run to the next and, thus, are computed once.

Distributed blade properties such as the blade's stiffness, weight, twist, and chord may be read into Module 1 at unevenly spaced points. The input data is interpolated to form sets of evenly spaced data. Another capability of this module is calculation of the blade's flapwise frequencies and modeshapes. This information is written to a file for examination by the user. From this information, the user can check the effects of mass and stiffness changes on blade frequencies and modeshapes.

We assume in this version of STRAP that both blades have identical mass, stiffness, twist, and chord distributions. In Module 1, mass, stiffness, and other matrices are calculated for only one blade. The effects of mass, pitch, and twist imbalances are not accounted for in this model.

The second module (STRAP2) calculates the rotor equations of motion and computes blade and low-speed shaft loads. The code calculates the rotor response to such input as gravity, windshear, tower shadow, yaw misalignment, and yaw rate. The response to these input is generally a steady-state one. The code is "started up" with initial values for the blade deflection and velocity. Once the blade reaches a steadystate trim solution, the blade loads are calculated and written to a file. The shaft loads are also calculated and written to a separate file.

The rotor response and load calculations resulting from time-dependent yaw motion or turbulent wind input is handled differently than the steady-state trim case. In those cases, a steady-state trim solution is calculated first, before the code enters the transient portion of the solution process. The results of this trim solution are then used as initial conditions for the transient analysis. After this trim solution is completed, the time clock is started, and the model is run, with the yaw function evaluated at each azimuth position. 
The yaw-function values for the initial yaw-angle displacement, yaw-angle amplitude, and maximum yaw velocity are set by the user during the model-run setup. Because the yaw function is given independently of the rotor model, the model solution from one rotor revolution to the next will not be steady state.

The number of revolutions to be run in the yaw solution is set by the user during run setup. After each rotor-revolution solution in the yaw routine, loads are computed and printed out.

The procedure for calculating rotor response and loads resulting from turbulent wind fluctuations is similar to the yaw solution procedure described above. The code first calculates rotor response for a trim (steadystate) solution. The computed values for blade deflection and velocity at zero azimuth (blade straight up) are then used as initial conditions for the transient analysis.

In order to run a turbulence analysis with STRAP, a file of turbulent, rotationally sampled wind data is needed. Such a file of data can be generated by such codes as the VEERS Three-Dimensional Wind Simulation (Veers, 1988). Additional details of this file generation and code execution with turbulence will be given in Sections 6.0 and 7.0. Example inputs to Module 1 are described next, including preparation of blade property data for an example turbine. First, some new code input parameters are described. 


\subsection{Description of New Input Variables}

For a review of old input variables to Module 1, the user is encouraged to review Table 3-15 in Wright, Buhl, and Thresher (1988). That table gives a description of all the input variables and lists the equations in which these variables were used. We will only describe herein those variables that are new to Module 1 of STRAP. Table 3-1 gives a description of these new variables.

The first new variables are HUBMAS, UNDSLG, and HUBDIS. These variables correspond to $\mathbf{M}_{\text {hub }}, \mathrm{u}$, and $\mathrm{d}$, as shown in Figure 2-2. Input of a nonzero value for UNDSLG ( $\mathrm{ft}$ ) moves the teeter axis away from the blade apex (point 0 ) to point A. If a positive value of UNDSLG ( $\mathrm{ft}$ ) is input, the teeter axis is moved by the distance $u$ downwind of point 0 (the blade apex).

One can input a concentrated hub mass $\left(\mathrm{M}_{\mathrm{hub}}\right)$ HUBMAS $\left(\mathrm{lb}-\mathrm{s}^{2} / \mathrm{ft}\right)$ a distance HUBDIS (d-ft) downwind of point 0 (the blade apex). The hub mass may consist of the mass of the hub that is not included in the calculation of the blade's distributed mass (by the input WEIGHT). The inclusion of a concentrated hub mass may move the rotor center of gravity relative to the teeter axis, which is important in the calculation of the rotor's response and loads as a result of gravity. HUBMAS is the mass of the hub only, with the blades removed. HUBDIS locates the center of gravity of the hub relative to the blade apex point (0).

The next input is the variable DELT3, which models the effects of delta-3 in teetered rotors. The effects of delta-3 are to couple flap motion with pitch motion of the blades. Figure 3-1 shows a teetered rotor with delta-3 $(\delta-3)$.

In the convention used here, we are located upwind of the blade looking downwind. As the top blade (\#1) teeters downwind (away from us), it will also pitch so that the leading edge pitches toward the wind. The blade is rotating clockwise.

It can be shown that the change in angle of attack (or pitch: $\Delta \Theta$ ) is related to the change in teeter $\Delta \beta$ by

$$
\Delta \Theta=\Delta \beta \sin \delta 3
$$

For most wind turbine rotors, DELT3 should be input as a positive value (in degrees). Input of a negative value will result in a destabilizing effect, resulting in poor convergence to a trim solution in Module 2.

The next six variables-BETA1, BETA2, $\mathrm{K}_{0}, \mathrm{~K}_{1}, \mathrm{~K}_{2}$, and CDAMP-allow modeling of teeter springs and damping. We will now describe these parameters.

In this analysis, there are three springs with stiffnesses $\mathrm{K}_{0}, \mathrm{~K}_{1}$, and $\mathrm{K}_{2}(\mathrm{ft}-\mathrm{lb} / \mathrm{rad}$ ) and one damper with damping constant CDAMP (ft-lb-s/rad). The angles BETA1 and BETA2 (deg) define those teeter angles at which one spring stops acting on the rotor and another one begins. All these springs are considered to be torsional springs with stiffnesses having units of $\mathrm{ft}-\mathrm{lb} / \mathrm{rad}$. The definitions are

$$
\begin{aligned}
K= & K_{0} \text { for } B<B_{1} \\
& K_{0}+K_{1} \text { for } B_{1}<B<B_{2} \\
& K_{0}+K_{2} \text { for } B>B_{2} \\
C= & \text { CDAMP for } B>B_{1}
\end{aligned}
$$

There may be a teeter bearing spring with stiffness $\mathrm{K}_{0}$ that acts on the rotor for all teeter angles. Once the blade reaches a certain angle, $B_{1}$ there may be a second spring with stiffness $K_{1}$. At some angle, $B_{2}$, 
Table 3-1. New Input Variable Description.

\begin{tabular}{|c|c|c|}
\hline Variable Name & Symbol & Description \\
\hline HUBMAS & $\mathbf{M}_{\text {hub }}$ & $\begin{array}{l}\text { Concentrated mass of only the } \\
\text { hub }\left(1 \mathrm{~b}-\mathrm{s}^{2} / \mathrm{ft} \text { ) (see Figure 2-2). }\right.\end{array}$ \\
\hline UNDSLG & $\mathbf{u}$ & $\begin{array}{l}\text { Distance of the teeter pin } \\
\text { downwind of the point of blades' } \\
\text { intersection (see Figure 2-2). }\end{array}$ \\
\hline HUBDIS & d & $\begin{array}{l}\text { Location of the hub center of } \\
\text { gravity downwind of the blades' } \\
\text { intersection point (ft) (see } \\
\text { Figure 2-2). }\end{array}$ \\
\hline DELT3 & $\delta-3$ & $\begin{array}{l}\text { Orientation of the teetering hinge } \\
\text { (see Figure } 3-1) \text { (deg). }\end{array}$ \\
\hline BETA1 & $\beta_{1}$ & $\begin{array}{l}\text { Angle of teeter (deg) at which the } \\
\text { rotor impacts first teeter stop (see } \\
\text { Figure 3-2). }\end{array}$ \\
\hline BETA2 & $\beta_{2}$ & $\begin{array}{l}\text { Angle of teeter (deg) at which } \\
\text { rotor impacts second teeter (hard) } \\
\text { stop (see Figure 3-2). }\end{array}$ \\
\hline K0 & $\mathrm{K}_{0}$ & $\begin{array}{l}\text { Stiffness of a teeter hinge spring } \\
\text { that acts on rotor for all teeter } \\
\text { angles (ft-lb/rad) (see Figure 3-2). }\end{array}$ \\
\hline K1 & $\mathrm{K}_{1}$ & $\begin{array}{l}\text { Stiffness of a spring (ft-lb/rad) } \\
\text { used to simulate stiffness of the } \\
\text { first teeter stop (see Figure 3-2). }\end{array}$ \\
\hline $\mathrm{K} 2$ & $\mathrm{~K}_{2}$ & $\begin{array}{l}\text { Stiffness of a spring (ft-lb/rad) } \\
\text { used to simulate siffness of the } \\
\text { second hard teeter stop (see } \\
\text { Figure 3-2). }\end{array}$ \\
\hline CDAMP & C & $\begin{array}{l}\text { Teeter damping, assumed to } \\
\text { occur for teeter angles greater } \\
\text { than BETA1 }\left(\beta_{1}\right) \text { (ft-lb-sec/rad). }\end{array}$ \\
\hline
\end{tabular}




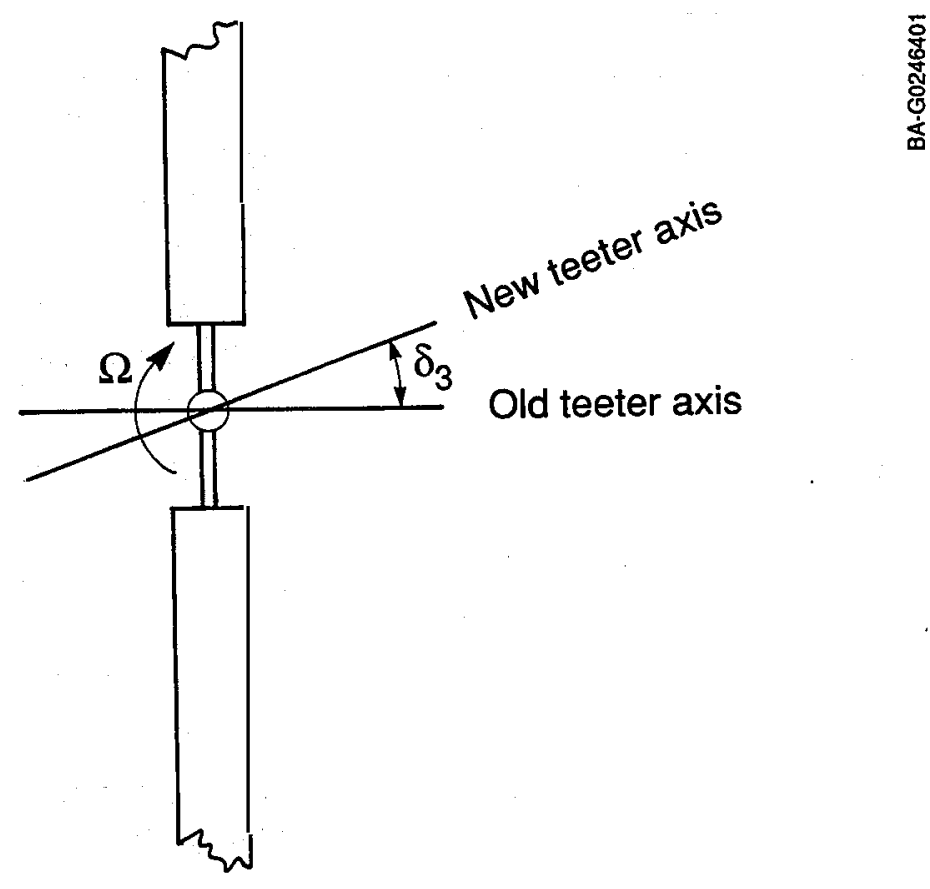

Figure 3-1. $\delta-3$ hinge (as observed looking downwind, rotor rotating clockwise)

the blade may hit a hard stop with stiffness $\mathrm{K}_{2}$. The damping (CDAMP) is considered to act for any teeter angles greater than $\beta_{1}$.

One note of caution is that use of a very high stiffness for $\mathrm{K}_{2}$ may cause longer run times for Module 2 . When running Module 2 with high values for $\mathrm{K}_{1}$ or $\mathrm{K}_{2}$, the user should set the run parameters STEPMX, EUERR, and TRMERR in Module 2 to one. This will cause smaller step sizes in the numeric integration process and improve chances for convergence to a trim solution.

Other new code input include NUMSCN, TIMINC, MSTAT, and STA. All these variables are related to the input of turbulent windspeed fluctuations from a separate file. This file of windspeed data must be produced using a separate code, such as the VEERS model (Veers, 1988).

NUMSCN is the number of lines of windspeed data in the turbulent wind input file. For example, a windspeed file may have 8,192 lines of data. TIMINC is the time increment between each line of wind data. An example file might be sampled in the VEERS model at $24 \mathrm{~Hz}$ so that TIMINC $=1 / 24 \mathrm{sec}$ $(.041667 \mathrm{sec})$. MSTAT is the number of blade stations at which turbulent wind data are input. At the present time, only a value of MSTAT $=2$ is input. STA represents the actual points on the rotor at which wind data are input, in this case, at 20 and $40 \mathrm{ft}$. These are the rotor 50\% and $100 \%$ radial locations. It is important to note that STA represents the distance from the rotor center of rotation and not the distance from the blade root. More details will be given in Sections 6.0 and 7.0 on turbulence input. 


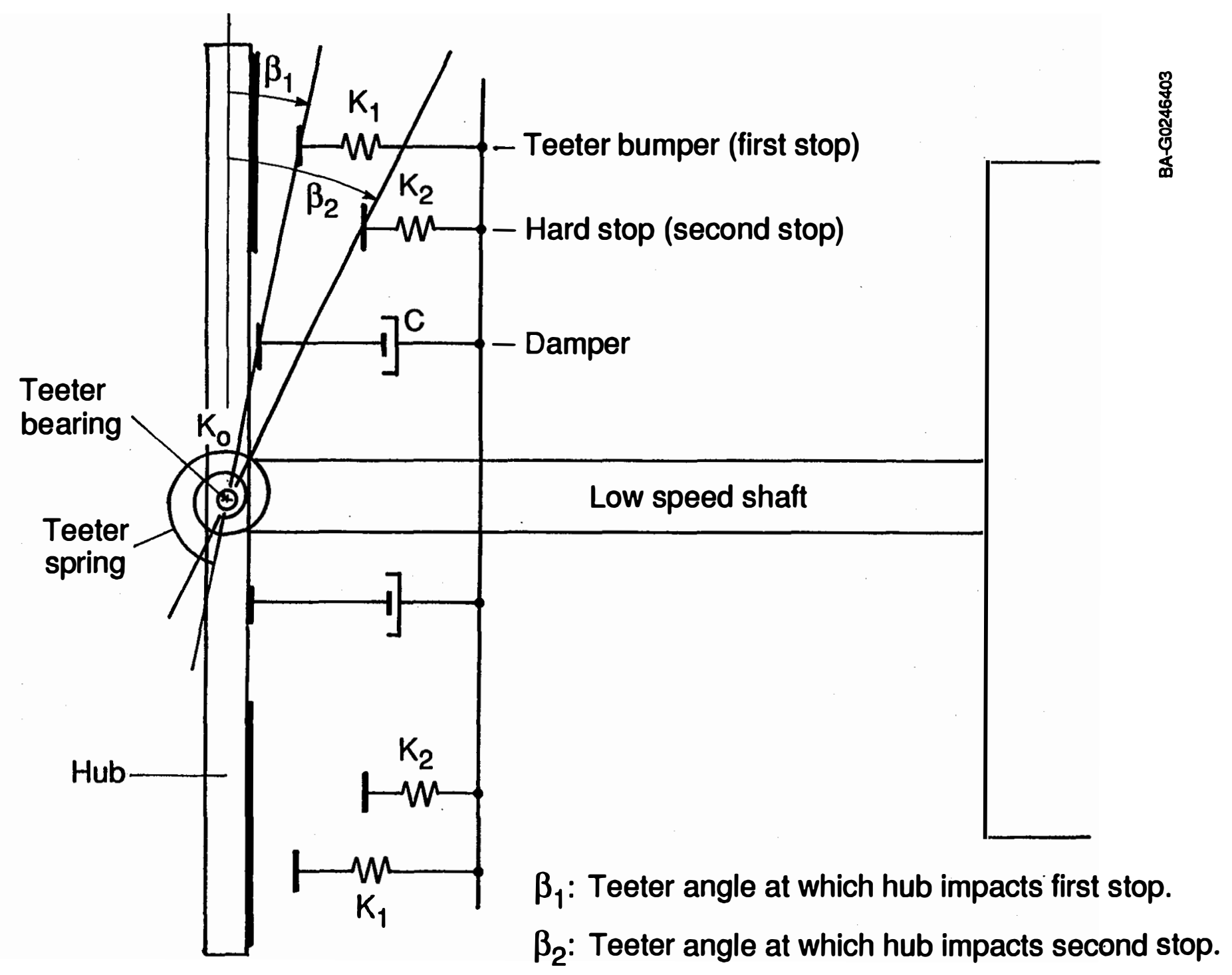

Figure 3-2. Illustration of the Teeter Springs and Damper Assembly 


\subsection{Example Turbine Description}

We will show the data input file preparation for an example two-bladed teetering hub test turbine. Before showing code input, the turbine will be described.

The ESI-80 wind turbine, shown in Figure 4-1, is a two-bladed, fixed-pitch, free-yaw, downwind, stallcontrolled turbine. It has a rotor diameter of $24 \mathrm{~m}(80 \mathrm{ft})$ and features wood epoxy composite rotor blades. These blades use LS(1)-04XX airfoils with thickness distribution and planform shown in Figure 4-2. This blade has a chord taper ratio of 2.2 beginning at the $30 \%$ blade radial station. Figure 4-2 also describes the linear trailing-edge-drop twist distribution of $4.0 \mathrm{deg}$. Blade stiffness and mass distributions are shown in Figures 4-3 and 4-4. The lift and drag profiles for the LS(1) are shown in Figure 4-5. The blade pitch is set to zero degrees measured at the $75 \%$ blade span. The rotor has a solidity of 0.035 and a coning angle of $7.0 \mathrm{deg}$ angled away from the tower. The teetered rotor has a delta-3 angle of zero degrees and rotates at a constant rotational speed of 60 RPM. Aerodynamically shaped tip vanes mounted at the blade tip perpendicular to the spanwise axis provide overspeed protection and assist in high-wind stops. Table 4-1 summarizes the major turbine specifications for the test turbine.

The distance from the yaw axis to the center of the hub is $6.79 \mathrm{ft}$. The teeter pin is $0.75-\mathrm{ft}$ downwind $(\mathrm{u}=0.75 \mathrm{ft})$ of the hub center. The mass of the hub, not modeled as blade mass, is $55.9 \mathrm{lb}-\mathrm{s}^{2} / \mathrm{ft}$ $\left(M_{\text {hub }}=55.9\right)$, located at the point where the blades intersect $(d=0)$. These inputs are important for locating the rotor's center of gravity with respect to the teeter pin.

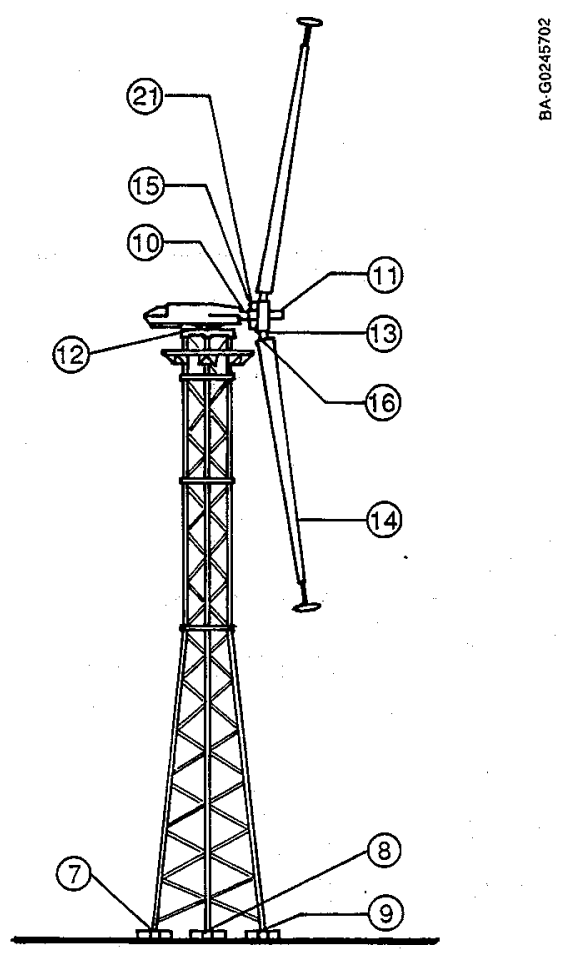

Figure 4-1. Illustration of example turbine 

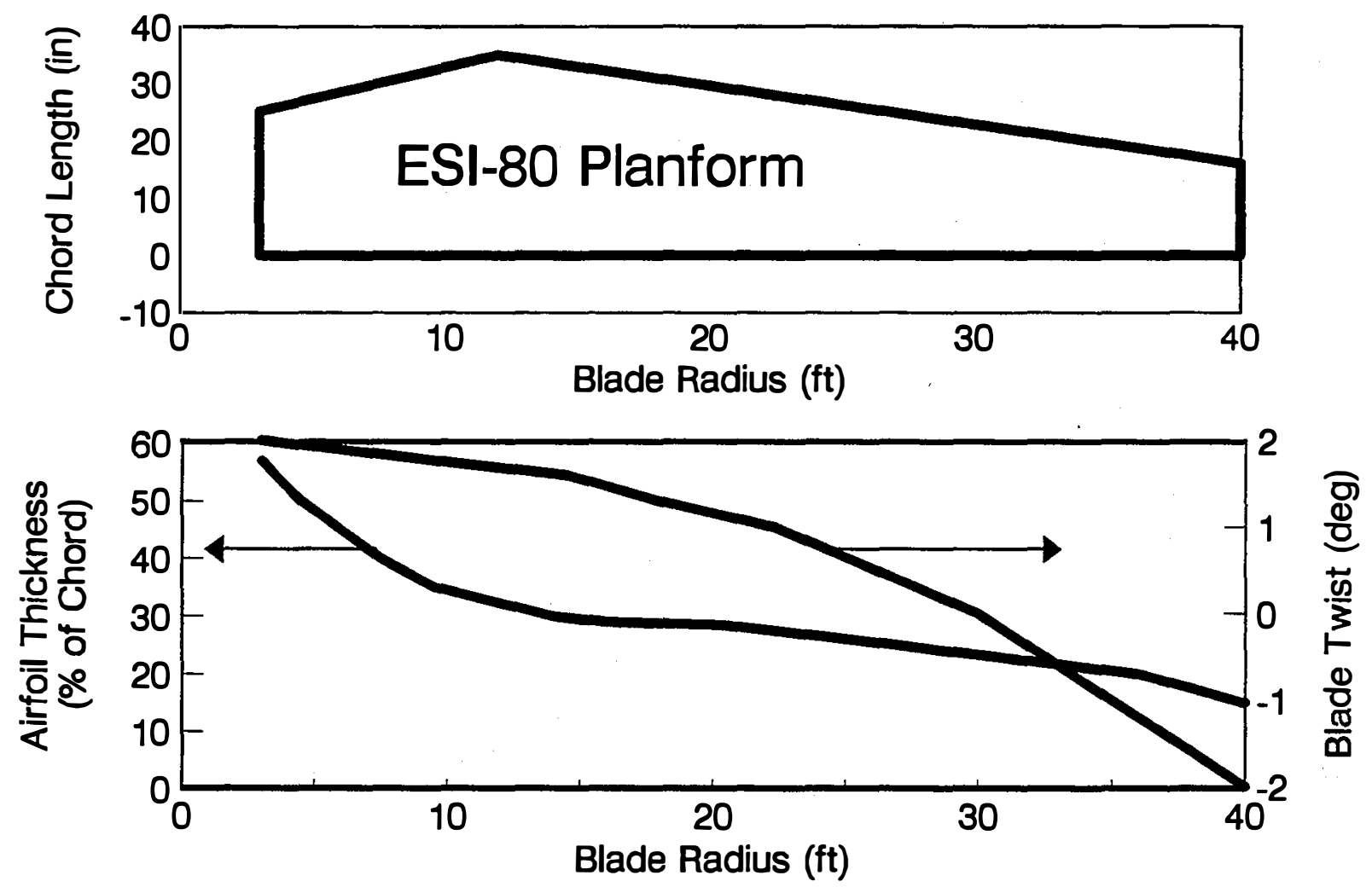

Figure 4-2. Turbine blade planform, thickness, and twist

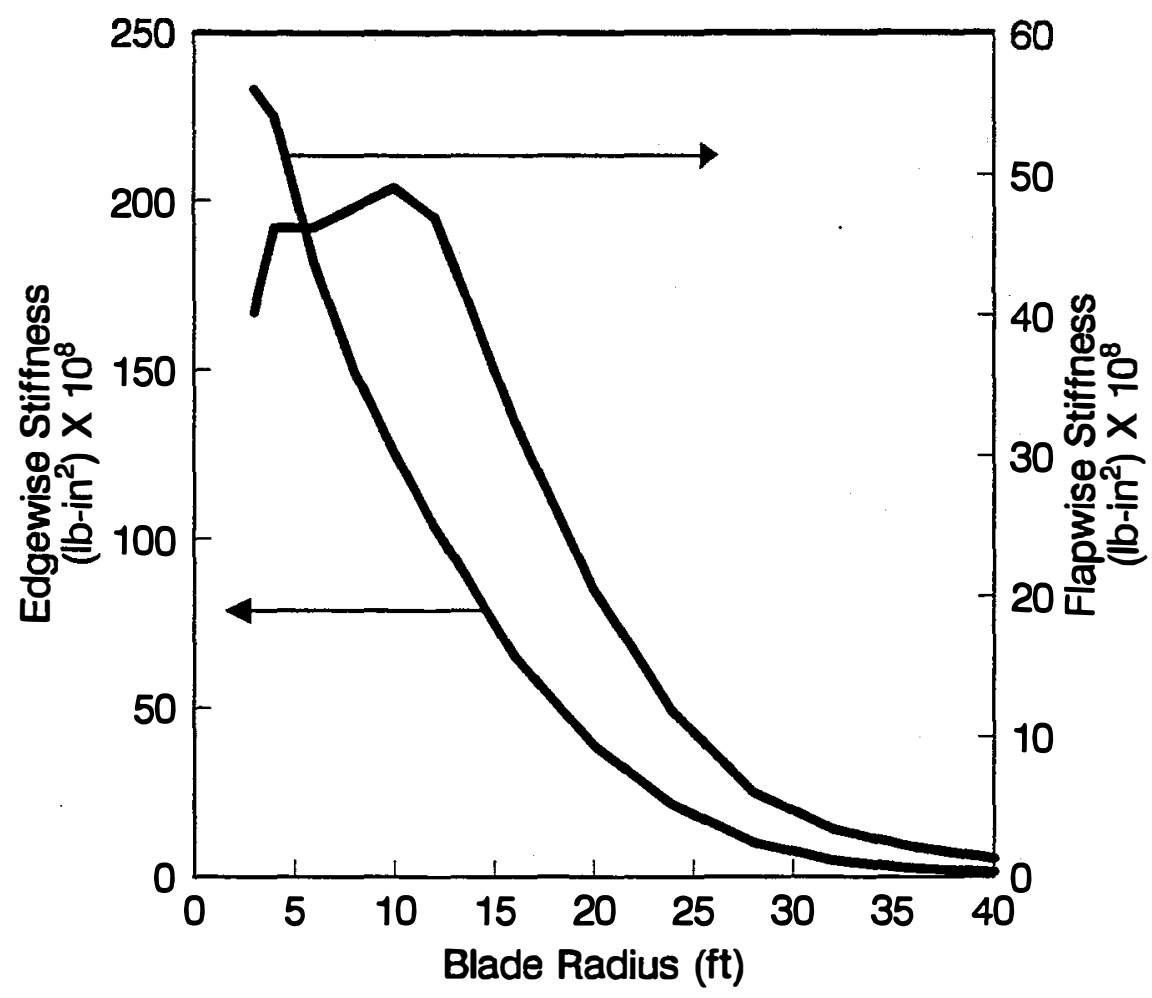

Figure 4-3. Turbine blade stiffness distribution 


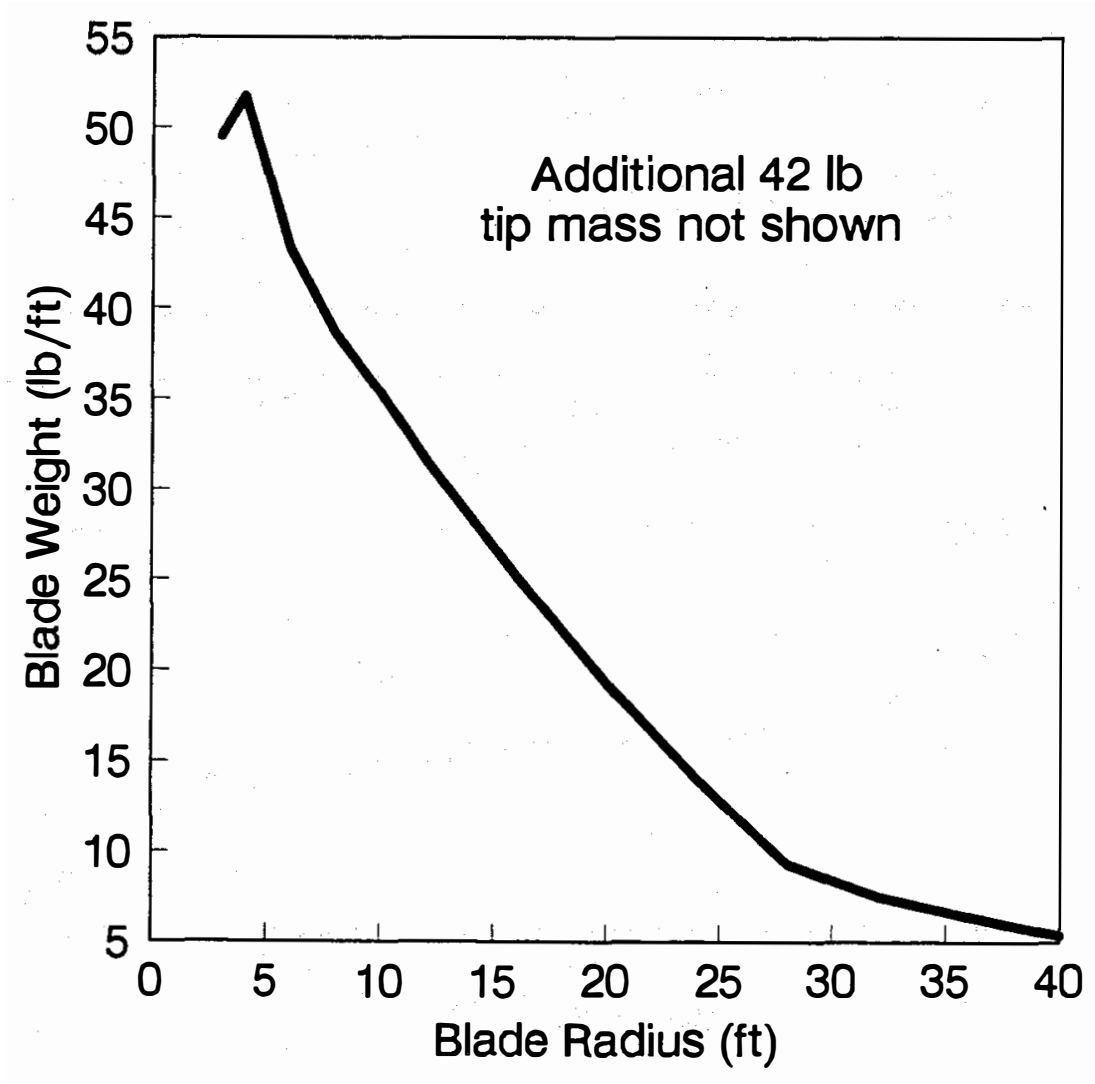

Figure 4-4. Turbine blade weight distribution

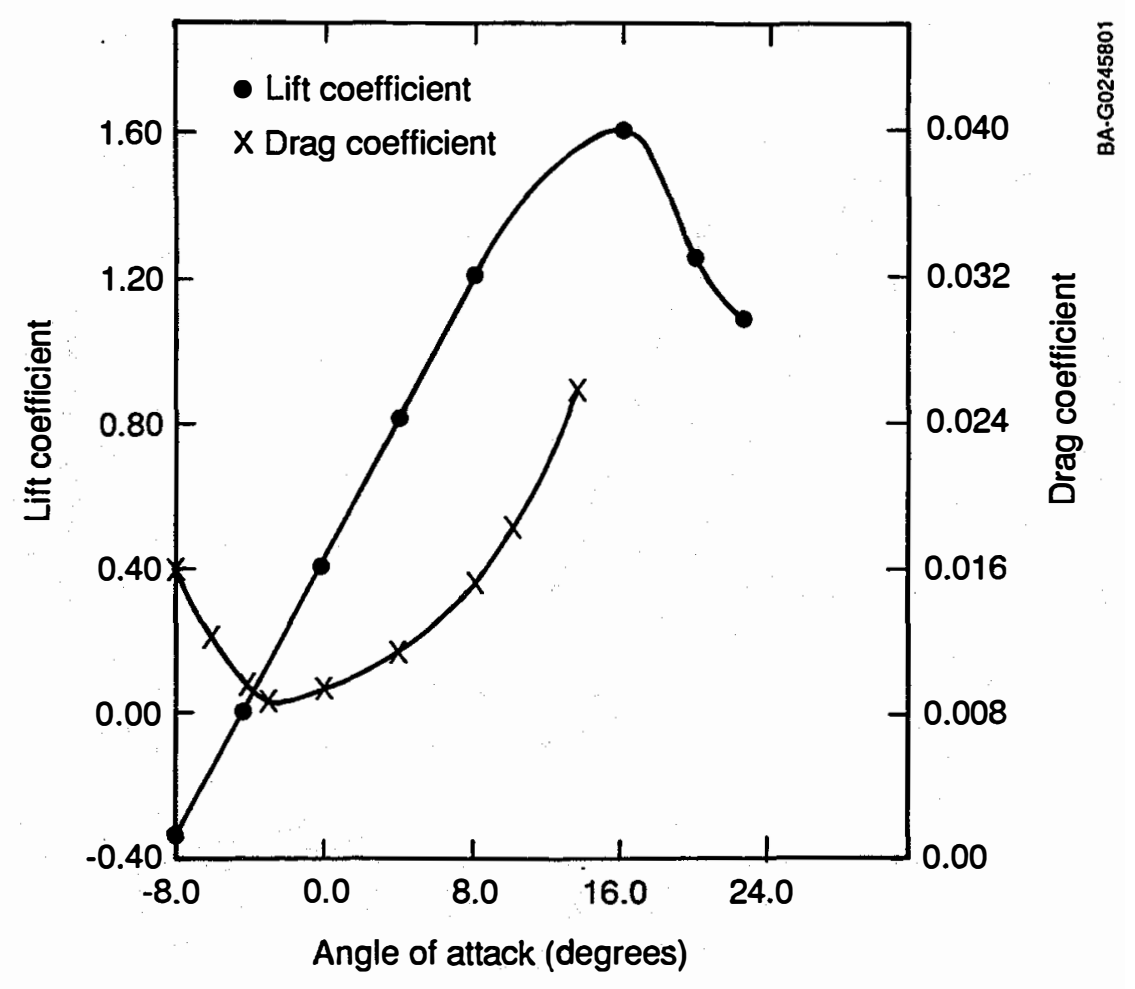

Figure 4-5. Lift profile for the LS(1) airfoil 
Table 4-1. Example Turbine Specifications

\begin{tabular}{|c|c|}
\hline Rated Power & $250 \mathrm{~kW}$ \\
\hline Rated Windspeed & $20.3 \mathrm{~m} / \mathrm{s}$ (45 mph) Rotor \\
\hline Diameter & $24.5 \mathrm{~m}(80 \mathrm{ft})$ \\
\hline Rotor Type & Teetered - Stall Control \\
\hline Rotor Orientation & Downwind \\
\hline Blade Construction & Wood Epoxy Composite \\
\hline Rotor Airfoil & NASA LS(1) 0417 \\
\hline Tip Speed & $77.9 \mathrm{~m} / \mathrm{s}(173 \mathrm{mph})$ \\
\hline Cut-in Windspeed & $5.9 \mathrm{~m} / \mathrm{s}(13 \mathrm{mph})$ \\
\hline Rotor RPM & $60 \mathrm{RPM}$ \\
\hline Generator RPM & $1,800 \mathrm{RPM}$ \\
\hline Generator Type & 300-kW Induction, 3 Phase \\
\hline Gearbox & Planetary \\
\hline Hub Height & $24.9 \mathrm{~m}(81.5 \mathrm{ft})$ \\
\hline Tower & Open - Truss \\
\hline Pitch Control & None \\
\hline Yaw & Passive \\
\hline Overspeed Control & Tip Vanes \\
\hline Total System Weight & $9,750 \mathrm{Kg}(21,500 \mathrm{lbs})$ \\
\hline Coning & $7 \mathrm{deg}$ \\
\hline \multicolumn{2}{|c|}{ Rotating Natural Frequencies } \\
\hline Teeter & $1 \mathrm{~Hz}$ \\
\hline First Symmetrical Flapwise & $2 \mathrm{~Hz}$ \\
\hline Second Symmetrical Flapwise & $7.8 \mathrm{~Hz}$ \\
\hline First Edgewise & $5.9 \mathrm{~Hz}$ \\
\hline Second Antisymmetrical Flapwise & $12 \mathrm{~Hz}$ \\
\hline
\end{tabular}




\subsection{Preparation of Input Data}

Appendix A, page 2 shows the basic input file to Module 1 for the turbine. We will now describe the basis for choosing these inputs for each variable.

\section{$\underline{\text { ALENTH }}(\mathrm{ft})$}

The distance from the tower centerline or yaw axis to the center of the hub (point where the two blades intersect) is $6.79 \mathrm{ft}$. Note that this is not necessarily the distance from the yaw axis to the teeter hinge. That distance is ALENTH + UNDLSG.

\section{$\underline{\text { ALPHAd }}(\mathrm{deg})$}

This variable represents the angle from the chord line to the zero lift line. This angle can be found from examination of the lift curve for the LS(1) airfoil shown in Figure 4-5 and represents the angle of attack at which $\mathrm{C}_{\mathrm{L}}$ is equal to zero. It is equal to $-4 \mathrm{deg}$ for this airfoil. We assume a constant ALPH $\phi$ for the entire blade span. Provisions for changing ALPH $\phi$ with blade span are not provided in this version of STRAP.

\section{$\underline{\mathrm{CHI}}(\mathrm{deg})$}

The angle $\mathrm{CHI}$ is the rotor shaft tilt, set equal to zero for this turbine. See Wright, Buhl, and Thresher (1988) for more details.

\section{CSUBMA}

This variable represents the airfoil pitching moment coefficient, set equal to 0.015 for this airfoil. For more details, see Wright, Buhl, and Thresher (1988), page 13. This input is not very important for calculation of flap-bending moments.

\section{DRGFRM}

This input variable is used in the calculation of airfoil drag coefficient, as given in Wright, Buhl, and Thresher (1988), equation 4-7, page 85 . It can be found by fitting a second-order equation of the form given in Wright, Buhl, and Thresher (1988) to the airfoil $C_{D}$ versus $C_{L}$ curve. For this airfoil, the value for DRGFRM was calculated as 0.0032 .

\section{$\underline{\text { HUBHT }}(\mathrm{ft})$}

The hub height of this turbine is $80 \mathrm{ft}$.

$\underline{\text { BETA申 }}(\mathrm{deg})$

The precone angle for this rotor is $7 \mathrm{deg}$.

\section{BLSHNK (ft)}

BLSHNK is defined as the length of blade shank measured from the blade root to the point where the airfoil section begins. A nonzero value for BLSHNK must always be input to this code. If the particular rotor being analyzed has a zero blade shank, set BLSNK to some small number (.01). Figure 5-1 clarifies the definition of this variable. The main purpose of this variable is to identify the portion of the blade 


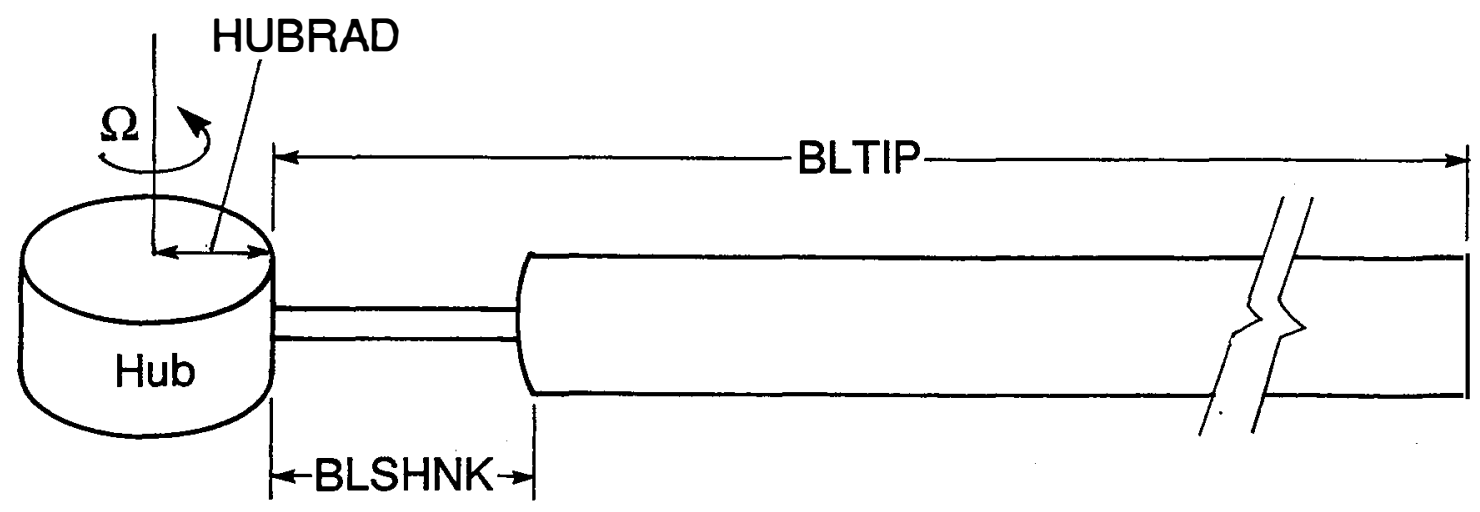

Figure 5-1. Illustration of rotor geometry

not producing aerodynamic lift. For this case, BLSHNK $=0.8 \mathrm{ft}$. Input of a zero for BLSHNK will cause an error in code execution.

$\underline{\operatorname{BLTIP}}(\mathrm{ft})$

This variable is the blade length, as measured from the blade root to the tip. This value will not, in general, be equal to the rotor radius (see Figure 5-1). For this turbine, BLTIP is set equal to $38 \mathrm{ft}$ because we have a hub radius of $2 \mathrm{ft}$. The hub is not considered as part of the blade.

$\underline{\text { HUBRAD }}(\mathrm{ft})$

HUBRAD is the distance from the axis of rotation to the blade root. For this rotor, HUBRAD is set equal to $2 \mathrm{ft}$. Figure 5-1 clarifies this input variable. In this version of the code the parameter HUBRAD does not need to be set to zero as in the previous version of FLAP (see Wright, Buhl, and Thresher, 1988, p 63).

\section{KSHADW}

This variable is used in the tower shadow model (see Wright, Buhl, and Thresher, 1988, pages 7, 88). It represents the number of oscillations in the tower shadow region. For this turbine, it is set to 3 because we have a three-legged truss tower.

\section{$\underline{\text { NBLADS }}$}

The number of blades is set to 2 .

\section{NPANEL}

This variable represents the number of points on the blade, beginning at the root and ending at the tip, in which distributed blade properties are input. We input properties at 11 points along the blade. The maximum value for NPANEL is 11 . 


\section{OMEGA (RPM)}

The rotor speed is $60 \mathrm{RPM}$.

PHIAMP (deg)

This variable is set to zero because we are not performing a yaw motion solution. See Ref. 4 for more details. This variable defines the yaw-motion amplitude in the time-dependent yaw solution.

$\underline{\text { PHIOMG }}(\mathrm{deg} / \mathrm{sec})$

This variable is also set to zero. This variable defines the steady yaw rate in the trim solution or the maximum yaw rate in a yaw-motion, time-dependent solution. (See Wright, Buhl, and Thresher, 1988).

$\underline{\text { PHI } \phi}(\mathrm{deg})$

This variable is set to +13 deg because the steady yaw error for this data case was 13 deg (see Wright, Buhl, and Thresher, 1988). See Wright and Butterfield (1992) for yaw error definition.

\section{PSIZER}

This variable represents the tower shadow half-width. The tower shadow is modeled as a pie-shaped sector centered about the tower centerline. The value of PSIZER was arbitrarily selected as 20 deg for this machine. For more details, see Wright, Buhl, and Thresher (1988), page 7.

\section{SHERXP}

The power law windshear relation given in Wright, Buhl, and Thresher (1988), page 7, had an exponent equal to 0.164 for this case. This value was determined from analysis of anemometer data at three heights, as described in Wright and Butterfield (1992).

\section{THETAP}

This variable represents the angle between the principal flapwise bending plane and the cone of rotor rotation. If the blade elastic flapping motion is considered to occur perpendicular to the rotor plane, then THETAP should be set to zero. If flapping motion is considered to occur about the section chord line at the blade root, then THETAP should be set to the angle between this chord line and the rotor cone of rotation.

For a single code run, the orientation of flapping is constant; it does not change from blade station to station because of changes in pretwist. To change the flapping direction for other stations, the code must be rerun with appropriate values of THETAP. All load and deflection results are referenced to this one axis system (the $\mathrm{X}_{\mathrm{p}}, \mathrm{Y}_{\mathrm{p}}, \mathrm{Z}_{\mathrm{p}}$ axes [Wright, Buhl, and Thresher, 1988]). For this case, we set THETAP to $4 \mathrm{deg}$ to reflect flapping about the root chord line. For more details, see Wright, Buhl, and Thresher (1988), pages 13 and 86.

\section{THETAT}

This variable represents the difference in twist between the reference station and the tip. Because the reference station was selected at the root, this twist difference is $4 \mathrm{deg}$. 


\section{WEIGHT}

We wanted to include the 42-lb tip weight in the blade's distributed weight input (provisions for inputing a concentrated tip weight are not included). We placed points at 36.0, 36.2, and $38.0 \mathrm{ft}$.

Distributed property data are interpolated in Module 1 to form a set of evenly spaced data. Between each value of XIEFT, the property data are being linearly interpolated. The point 36.2 was chosen to allow the weight to change rapidly.

The other values chosen for WEIGHT were based on the blade's weight distribution, shown in Figure 4-4.

$\underline{\text { AEIARE }}\left(\times 10^{6} \mathrm{lb}-\mathrm{ft}^{2}\right)$

Figure 4-3 shows the stiffness distributions for this airfoil. Input of values from this plot result in overprediction of the blade's flapwise bending frequencies. We deliberately reduced these values uniformly along the blade so that the predicted flapwise frequencies will agree with test data. It is known that the first symmetric flapwise frequency is approximately $2 \mathrm{~Hz}$. Values taken directly from Figure 4-3 result in overprediction of the frequencies. The user can now try different mass and stiffness distributions, run Module 1, and see what effect these changes have on predicted frequencies. We deliberately reduced the blade's stiffness distribution.

We did not adjust the blade's weight distribution because this parameter is so important for correct calculation of blade centrifugal and gravitational loads.

\section{AIEMASS, AIFMASS, AOFFST, AESBAC}

These input variables are set to zero. We did not perform a thorough investigation of the effects of these parameters on blade loads and response. The variables AOFFST and AESBAC may have some effect on the torsional moments predicted by STRAP. We do not think these input parameters have a big effect on flapwise bending moments, however.

\section{$\underline{\text { ACHORD }}$}

This variable is input directly from the information given in Figure 4-2.

\section{$\underline{\text { ATWIST }}$}

This variable represents the blade's built-in twist distribution. It is input from the information given in Figure 4-2. It is important to note that these values must be adjusted in order to obtain zero twist at the tip; i.e., ATWIST $(11)=0$. The input variable ATWIST has the blade tip as its zero reference point instead of the 75\% span, as seen in Figure 4-2.

\section{ACLALF, ACDZER}

ACLALF is the lift curve slope. It is allowed to vary along the span. These values were input from information on the LS(1) airfoil.

ACDZER is the drag coefficient at zero lift. It is used in the calculation of drag coefficient (Wright, Buhl, and Thresher, 1988). It is also allowed to vary along the span. 


\section{NUMSCN, TIMINC, MSTAT, STA}

These four variables provide information necessary for input of turbulent wind fluctuations to STRAP. NUMSCN was input as 8,192 to reflect 8,192 lines of data in the turbulent windspeed file. TIMINC is set to .041667 because the wind data are sampled at $24 \mathrm{~Hz}$. The number of blade stations at which windspeed data are input is two. Note: The hub center windspeed data will be read in also. MSTAT represents the number of blade stations outboard of the hub center at which turbulent wind input are to be read. STA gives the positions (as measured from the center of rotor rotation) of these points, here set to 20 and $40 \mathrm{ft}$, which is $50 \%$ and $100 \%$ of the rotor radius. These inputs are seen in the input file in Appendix A. 


\subsection{Preparation of Turbulent Wind Input Data}

If the user is not interested in running a case with turbulent windspeed input, then this section can be skipped. The STRAP code can be run for deterministic cases only by simply choosing the (Q) option after the trim solution.

In order to run a turbulent windspeed case, a separate file of turbulent windspeed data is needed for input to STRAP. It is assumed that the user must run a separate turbulence wind simulation model such as Veers (1988). Some specific details on the VEERS model will now be given as well as the structure of the turbulence input file to STRAP.

We recommend the use of Veers (1988) for generation of turbulent windspeed input for this rotor. We will henceforth refer to Veers (1988) as the VEERS Three-Dimensional Wind Simulation or, simply, the VEERS model. The reason we recommend this code is that it can be used to generate turbulent windspeed data needed for both blades of a two-bladed teetering hub rotor. With this model, a full threecomponent field of turbulence is not calculated, only the longitudinal, "along wind," component.

This simulation method is used to obtain rotationally sampled windspeeds (Veers, 1988). We simulate the windspeed at five points of the rotating two-bladed rotor: the $50 \%$ rotor radius location on blade 1 , the $100 \%$ rotor radius for blade 1 , the $50 \%$ rotor radius for blade 2 , the $100 \%$ rotor radius for blade 2 , and the windspeed for the center of rotor rotation.

In the VEERS code, various spectral models for the fixed-point power spectral density calculations can be chosen (Veers, 1988). We usually choose the Solari model. The coherence model is the exponential model with some modifications (Veers, 1988). Input to the model include mean windspeed, rotor speed, number of blades, turbulence intensity, terrain surface roughness, and coherence decrement. For more information, see Veers (1988).

Another input to this model is the number of equally spaced azimuth points in which windspeed data are to be simulated. This parameter will dictate the sampling rate of the generated, rotationally sampled windspeed data. From information that we have obtained from Winkelaar (1991), we usually set this parameter to a high value, in our case, 24 points around the azimuth. This will result in a turbulent windspeed file consisting of six columns of data generated at $24 \mathrm{~Hz}$. The time increment between digitized data is, thus, approximately $0.0417 \mathrm{sec}$.

In the output file from the VEERS model, the first column is rotor azimuth angle (deg), the second is windspeed at $50 \%$ rotor radius on blade 1 , the third is windspeed at $100 \%$ rotor radius on blade 1 , the fourth is windspeed at $50 \%$ rotor radius on blade 2 , the fifth is windspeed at $100 \%$ rotor radius on blade 2 , and the sixth is the center of hub windspeed. The file contains 8,192 lines of data sampled at $24 \mathrm{~Hz}$. This file represents $341 \mathrm{sec}$ of real-time wind data. The number of lines of data in the file can be set in the VEERS model input file. We usually want this number to be some power of two ( $2^{13}$ in this case) so that we can later perform power spectral density calculations on the computed loads and response output. The STRAP code will generate corresponding loads and response output corresponding to each line of windspeed data. An example output file from VEERS is shown in Appendix F. 


\subsection{Input of Turbulence Data to STRAP}

At this time, we will describe how these turbulent windspeed data are read into STRAP and how they are used.

In order to run a turbulent windspeed case, the STRAP code must first be run in order to determine a trim solution. The code then uses the computed values of blade deflection and velocity at zero azimuth (blade at 12 o'clock) as initial conditions for this process. An example interactive execution will be shown in the next section.

One major change included in STRAP and not included in FLAP, version 2.01, is a subroutine named TRBCLC. After the code finishes with its trim solution calculations, it enters this subroutine, if so chosen by the user. It is in this subroutine that the turbulent windspeed data generated by Veers is read into STRAP.

We must now describe the two types of interpolation of this windspeed data that are occurring in STRAP. One type is with respect to rotor azimuth, and the other is along the blade radial station locations.

To describe the first type, we must remember that the STRAP code does not increment the rotor azimuth angle in equal azimuth steps. They can vary from point to point because of the use of the Euler Predictor Corrector numeric integration procedure (Wright, Buhl, and Thresher, 1988). The windspeed data generated from VEERS (Veers, 1988) is generated at equal time steps. The main purpose of subroutine TRBCLC is to interpolate the data in order to provide approximate wind input at intermediate rotor azimuth locations. Linear interpolation is used in this subroutine.

Appendix D gives a listing of STRAP2. Subroutine TRBCLC is on page D-92. Upon entering this subroutine, the user will be prompted for the name of the windspeed input file and asked whether the windspeed data are in English (ft/sec) or metric $(\mathrm{m} / \mathrm{sec})$ units. He or she is then asked for a file name that contains load and response predictions. These predictions are not written in the RESULTS.DAT file formed previously.

The code then reads one line of wind input data at a time. For the first pass through this subroutine, before the first line of windspeed data is read, the initial azimuth angle is set to zero degrees, and the initial values for the two windspeeds on each blade and the hub center windspeed are set equal to VHUB. The first line of data is then read. The azimuth angle is then incremented, and wind input are then determined by linear interpolation for this intermediate azimuth angle. The values calculated at this intermediate azimuth are stored in the variables VYNOW1(1), VYNOW1(2), VYNOW2(1), VYNOW2(2), and VYNOWH. These variables represent the interpolated values of the two velocities on each blade and the hub center wind velocity at the present azimuth angle. The two end-point values, used in the linear interpolation process, represent the last and most recent values of windspeed read from the input file. These values are represented by the variables VYSAV1(I,J), VYSAV2(I,J), and VYSAVH(I). The integer I can be either 0 or 1 , corresponding to old or new. The integer $\mathrm{J}$ is equal to 1 or 2 , corresponding to windspeed at the $50 \%$ or $100 \%$ radial location. The variable VYSAVH(I) is the hub center windspeed.

The five interpolated windspeeds are ultimately passed to a subroutine name WINDVL. In this subroutine, values of turbulent windspeed are calculated for intermediate blade radial stations.

The STRAP code calculates blade aerodynamic forces at 21 equally spaced blade stations, beginning at the root and ending at the tip. In this subroutine, we take the five values of windspeed passed from subroutine TRBCLC and calculate interpolated values for intermediate blade radial stations. We then have 
an array containing 21 equally spaced windspeed inputs for a blade. These values are saved in the array DELTVY(I); see Appendix D, page D-89 for Subroutine WINDVL.

This process of azimuth interpolation in subroutine TRBCLC and blade radial station interpolation in WINDVL is continued for each iteration in the numeric process. The code will calculate teeter and elastic flap deflections, velocity, and accelerations at every single iteration point. The code calculates and saves loads only at those azimuth positions corresponding to the azimuth values read in from the turbulent windspeed file.

Results written to the user-designated output file include azimuth angle, teeter angle, blade 1 loads and deflection information, blade 2 loads and deflection information, and low-speed rotor shaft loads (in the rotating coordinate system) at the hub center. The user can modify the write statement in subroutine TRBCLC to write out all or part of these results. This file will contain the same number of lines of output results as the number of lines of data in the windspeed input file.

Some remarks should be made on proper execution of the trim solution portion of this process. The file produced by the VEERS model contains windspeed data derived from three parts: (1) the mean hub height windspeed, (2) the variation of windspeed as a result of windshear, and (3) the variation as a result of stochastic effects. The second part may be zero if the VEERS model is run with a zero windshear exponent.

Care must be taken to run the trim solution in STRAP using the correct values of VHUB and SHERXP. The variable VHUB, in Module 2 of STRAP, should be set to the value of hub height windspeed as calculated in the VEERS model. The mean windspeed input to the VEERS model is the mean windspeed at a 10-m height (Veers, 1988). This will not necessarily be equal to the hub height windspeed. Hub height may be at a different level. The equivalent hub height windspeed from the VEERS model can be calculated from a knowledge of the windspeed input at $10 \mathrm{~m}$ and the power law shear exponent. Once this value is known, STRAP SHOULD BE RUN WITH VHUB SET TO THIS VALUE IN FEET/SECOND.

If the VEERS model is run with a nonzero shear exponent, then the STRAP code should be run with SHERXP set to zero. The wind data generated by the VEERS model already contain the effect of windshear. Input of a nonzero SHERXP in STRAP will result in windshear overprediction. To be safe, we set SHERXP, at the beginning of subroutine TRBCLC, to zero.

An example interactive run of Modules 1 and 2 will now be given. 


\subsection{Example Interactive Run}

\subsection{Module 1-STRAP1}

Please see Appendix A for a printout of this interactive run. Upon code execution, the user is first prompted for the input file name, here referred to as TURBN.DAT. Upon typing in the input file name, the user is prompted for the name of the run-data file name. This file is written by STRAP1 and contains the data to be read by STRAP2.

All the input data are echoed out to allow the user to check all inputs. At this point, the user is not able to change input during execution of Module 1 . The changes must be made to the input file and then rerun.

After all the input data have been echoed out, the user is prompted for the name of a file for writing the blade's modeshape and natural frequency data. This file is different than the run-data file. The purpose of this modeshape file is to allow the user to examine resulting flapwise natural frequencies and modeshapes. It is not read by STRAP2, and its only purpose is to examine frequency results.

Finally, the user is asked if he or she wants to process another data file; in which case, he or she answers yes or no. The code then responds by repeating the previous steps or stating "STRAP Terminated Normally." This signals the end of STRAP1 execution. A listing of this interactive session is shown in Appendix A. A listing of the run-data file and the modeshape file is also shown.

\subsection{Module 2-STRAP2}

Upon executing STRAP2, the user is presented with a menu of options shown in the interactive run listing in Appendix B. The options include (R)ead in a data file, (S)et up and run the model, (D)iagnostic run, (T)urbulence run, and (Q)uit.

The user first chooses the (R) option and reads in the run-data file produced by Module 1 (STRAP1). After this step, the user selects option (S). Upon selecting (S), a list of free variables will be given. The user is free to change any of these variables. At this stage, the user selects the number of degrees of freedom to be included in the analysis. The variable NSHAPS sets the number of modes (from 1 to 4) to be used in the analysis. We suggest that NSHAPS be set to at least 2 so that the rigid-body teeter mode and the first symmetric bending mode are used in the analysis. For the example turbine described earlier, final code runs should use a value of 4 for NSHAPS because the second symmetric bending mode at a frequency of $8 \mathrm{~Hz}$ (8 times the rotor rotation speed) gets highly excited by effects for this rotor such as windshear, tower shadow, and turbulence (Wright and Butterfield, 1992).

After changing any of the free variables, the user is given the option of changing the run parameters. We suggest changing the variables STEPMX and EUERR under certain conditions. See Veers (1988) for the definition of these two variables. The default parameters (STEPMX $=10$, EUERR $=10$ ) can be used for initial runs, when only one or two modes are used, at low windspeeds in nonstalled conditions. When more modes are used or in cases involving high angles of attack (stalled flow), severe tower shadow input teeter stop impacts, etc., these parameters should be set to 1. Although the code will take longer to run, decreasing these input parameters will help the code to converge to a solution. The small step size is necessary when the blade is encountering highly nonlinear operating conditions, such as stalled flow, and abrupt changes because of tower shadow or teeter stop impacts. 
For high-windspeed cases, the code may take 20 to 30 trim iterations before a solution is reached. This occurs because of the decrease in blade aerodynamic damping that result from high angle-of-attack conditions.

We also suggest setting the Trace Flag in the set of run parameters to TRUE. This allows printout of blade deflection, velocity, acceleration, and other items in the RESULTS.DAT file. From this information, the user can monitor problems that may be occurning during code execution.

Another variable in this list of parameters is NYAW. If the user wants to run a time-dependent yaw solution, NYAW should be set to some integer number greater than zero. The variable PHIAMP in the free variable list must also be greater than zero (see Wright, Buhl, and Thresher, 1988).

NYAW represents the number of rotor revolutions to be performed during the yaw solution. The code will first compute a trim solution and then perform a transient analysis using the time-dependent yaw solution. The solution is completed at the end of NYAW revolutions.

After reviewing and/or changing the set of run parameters, the user is prompted for the name of the RESULTS.DAT file. This file will contain the rotor teeter, blade deflection, slope, and velocity results as well as all the blade's force and moment values. These results are given as a function of blade azimuth angle as well as blade radial station.

An option we have added to STRAP is the ability to input initial values for the blade's teeter and deflection data. The number of required input will depend on the value for NSHAPS, set in the free variable list. These initial values are assumed to be for a blade azimuth angle of 270 deg.

We have found this capability useful for speeding convergence for cases involving stall or other conditions requiring a long run time. The user can first run the code with the TRACEF flag (in the run parameters) set to TRUE with the code default initial conditions. After 10 trim iterations, the user can stop execution of the code. The user can then examine the RESULTS.DAT file and find the last values for the blade deflections at the azimuth angle of $270 \mathrm{deg}$. The code can then be rerun with these values used as initial values for the rotor teeter and blade deflection values. These steps will help speed convergence for cases involving high windspeeds or other nonlinear phenomena. Once the code obtains a satisfied trim condition, the code prompts the user for a Fourier analysis of result data.

The nine result items are then listed, and the user chooses which item (one at a time) to be Fourier analyzed. The code then determines Fourier cosine and sine coefficients and writes them in table form to the RESULTS.DAT file. A RESULTS.DAT file corresponding to the interactive run is shown in Appendix B.

After this, the name of a shaft load output file is asked for. The shaft loads for the trim runs are written to a separate file. A printout of this file for this interactive run is also shown in Appendix B.

After this, the user is asked if he or she wants to make another run with these data. If the answer is yes, the previous steps are repeated. If the answer is no, the user is returned to the operations menu.

One option that has not been described is (D). This option can only be performed after a trim solution has been performed. Upon execution of this option, items such as angle of attack, lift, and drag will be written to a file named DIAGNOS.DAT. This information is presented for various blade stations as well as rotor azimuth positions. 
The other option to be described is (T). This option must also be chosen only after a trim solution has been performed. In this case, we can see that a turbulence analysis was performed. The turbulent windspeed input file was named WIND-2.DAT. These windspeed data were in metric units (meters/ seconds). The turbulence load output file was named LOADS-2.DAT. If the user wants to bypass the turbulence analysis, then the (Q) option should be chosen before the $(T)$ option. This will halt code execution. 


\subsection{Conclusions}

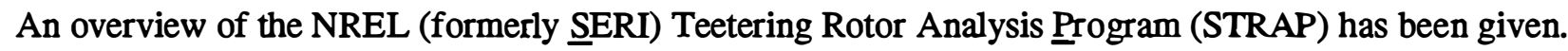
New input to the code have been described. An example two-bladed teetering hub wind turbine was described. Preparation of code input and an example code interactive run for this turbine were shown. Code and interactive run listings are given in the appendices. 


\subsection{References}

Veers, P. S., 1988, Three-Dimensional Wind Simulation, SAND 88-0152, Sandia National Laboratories, Albuquerque, New Mexico.

Winkelaar, D., 1991, First Three-Dimensional Wind Simulation and the Predictions of Stochastic Blade Loads, presented at the Tenth ASME Wind Energy Symposium, Houston, Texas.

Wright, A. D., and Butterfield, C. P., 1992, The NREL Teetering Hub Rotor Code: Final Results and Conclusions, presented at the Eleventh ASME Wind Symposium, Houston, Texas.

Wright, A. D., Buhl, M. L., and Thresher, R. W., 1988, FLAP Code Development and Validation, SERITR-217-3125, National Renewable Energy Laboratory, Golden, Colorado. 


\subsection{Acknowledgments}

The author would like to thank C. P. Butterfield of the National Renewable Energy Laboratory for assisting in analysis and interpretation of test results from the example turbine discussed in this report. Thanks also goes to Dr. Paul Veers of Sandia National Laboratories for many helpful suggestions on the correct use of the turbulence model. Thanks go to Lynn Starr and Rick Clyne for preparation and editing of this manuscript, respectively. 
Appendix A

Module 1 Input File, Interactive

Run Listing, and Output Files

A-1 


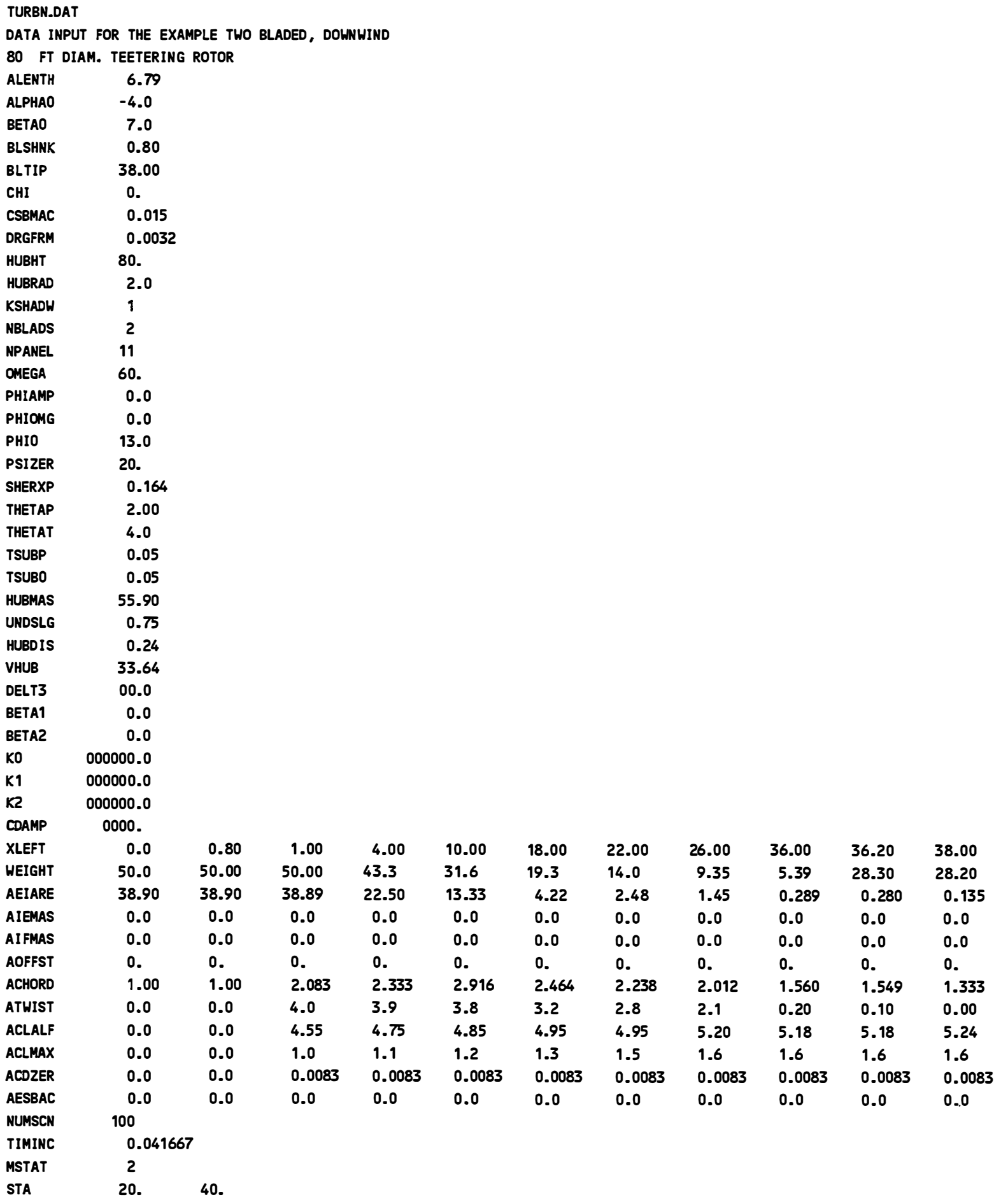


Program For Analysis of a 2-Bladed Teetering Hub Rotor Dynamic Response and Loads Analys is

MODULE 1- STRAP1

Subroutine INPUT: Input and process new blade and turbine data.

Enter name of input data file >

turbn.dat

Enter name of output data file >

runb.dat

TURBN.DAT

DATA INPUT FOR THE EXAMPLE TWO BLADED, DOWNWIND 80 FT DIAM. TEETERING ROTOR

Parameter values:

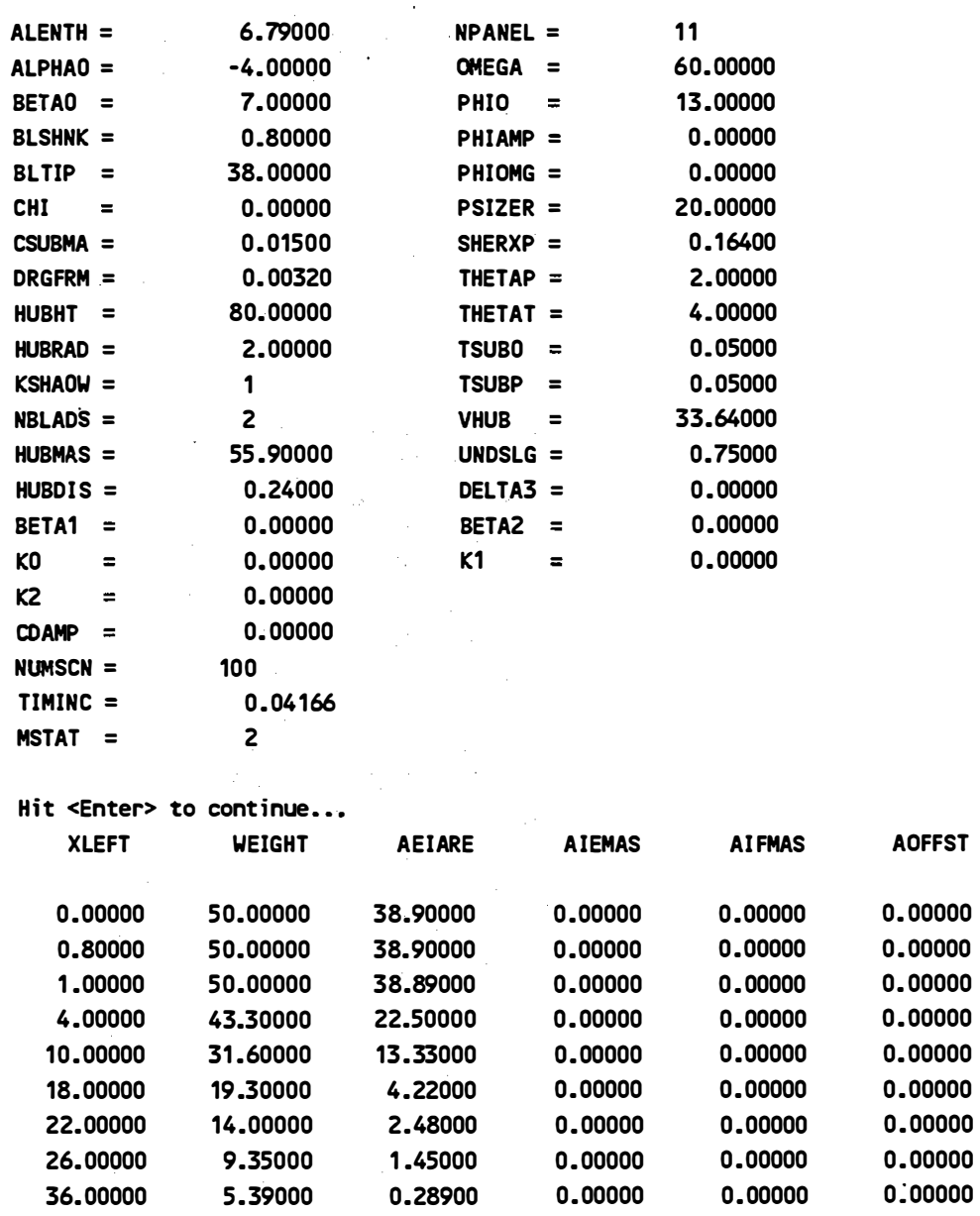




$\begin{array}{llllll}36.20000 & 28.30000 & 0.28000 & 0.00000 & 0.00000 & 0.00000 \\ 38.00000 & 28.20000 & 0.13500 & 0.00000 & 0.00000 & 0.00000\end{array}$

Hit <Enter> to continue...

$\begin{array}{llllll}\text { ACHORD } & \text { ATHIST } & \text { ALCALF } & \text { ACLMAX } & \text { ACOZER } & \text { AESBAC } \\ & & & & & \\ 1.00000 & 0.00000 & 0.00000 & 0.00000 & 0.00000 & 0.00000 \\ 1.00000 & 0.00000 & 0.00000 & 0.00000 & 0.00000 & 0.00000 \\ 2.08300 & 4.00000 & 4.55000 & 1.00000 & 0.00830 & 0.00000 \\ 2.33300 & 3.90000 & 4.75000 & 1.10000 & 0.00830 & 0.00000 \\ 2.91600 & 3.80000 & 4.85000 & 1.20000 & 0.00830 & 0.00000 \\ 2.46400 & 3.20000 & 4.95000 & 1.30000 & 0.00830 & 0.00000 \\ 2.23800 & 2.80000 & 4.95000 & 1.50000 & 0.00830 & 0.00000 \\ 2.01200 & 2.10000 & 5.20000 & 1.60000 & 0.00830 & 0.00000 \\ 1.56000 & 0.20000 & 5.18000 & 1.60000 & 0.00830 & 0.00000 \\ 1.54900 & 0.10000 & 5.18000 & 1.60000 & 0.00830 & 0.00000 \\ 1.33300 & 0.00000 & 5.24000 & 1.60000 & 0.00830 & 0.00000\end{array}$

Hit <Enter> to continue...

STA-

20.000

40.000

Hit <Enter> to continue...

Enter name of modeshape and frequency file >

MODESb.DAT

Data for MOOULE 2 have been written to RUNB.DAT.

Do you want to process another data file? $\left(Y_{,}=N\right)>$

STRAP1 terminated normally. 
THIS IS THE MODESHAPE AND FREQUENCY FILE PRODUCEd BY MODULE 1:

\begin{tabular}{|c|c|c|c|c|}
\hline \multicolumn{5}{|c|}{ FREQUENCIES $=($ RADIANS/SEC $)$} \\
\hline 6.28319 & 15.4516 & 30.0608 & 50.6140 & \\
\hline \multicolumn{5}{|l|}{ Modeshapes: } \\
\hline 1 & $0.499915 E-01$ & 0.000000 & $-0.128996 \mathrm{E}-01$ & 0.000000 \\
\hline 11 & $0.974451 E-01$ & $0.479990 E-03$ & $-0.247922 E-01$ & $-0.470760 \mathrm{E}-03$ \\
\hline 21 & 0.144827 & $0.226373 E-02$ & $-0.364379 E-01$ & $-0.172893 E-02$ \\
\hline 31 & 0.192138 & $0.560398 E-02$ & $-0.480739 E-01$ & $-0.376290 E-02$ \\
\hline 41 & 0.239369 & $0.105679 E-01$ & $-0.594390 E-01$ & $-0.668386 E-02$ \\
\hline 51 & 0.286509 & $0.171897 E-01$ & $-0.699322 E-01$ & $-0.105695 E-01$ \\
\hline 61 & 0.333544 & $0.255666 E-01$ & $-0.787526 E-01$ & $-0.153675 E-01$ \\
\hline 71 & 0.380458 & $0.359055 E-01$ & $-0.850203 E-01$ & $-0.208500 E-01$ \\
\hline 81 & 0.427239 & $0.485312 E-01$ & $-0.878786 E-01$ & $-0.266104 E-01$ \\
\hline 91 & 0.473877 & $0.638611 E-01$ & $-0.865778 E-01$ & $-0.320938 E-01$ \\
\hline 101 & 0.520367 & $0.823587 E-01$ & $-0.805395 E-01$ & $-0.366515 E-01$ \\
\hline 111 & 0.566707 & 0.104471 & $-0.694031 E-01$ & $-0.396127 E-01$ \\
\hline 121 & 0.612903 & 0.130561 & $-0.530526 E-01$ & $-0.403623 E-01$ \\
\hline 131 & 0.658964 & 0.160841 & $-0.316252 E-01$ & $-0.384173 E-01$ \\
\hline 141 & 0.704903 & 0.195319 & $-0.550104 E-02$ & $-0.334936 E-01$ \\
\hline 151 & 0.750740 & 0.233762 & $0.247261 E-01$ & $-0.255528 E-01$ \\
\hline 161 & 0.796495 & 0.275687 & $0.582962 E-01$ & $-0.148218 E-01$ \\
\hline 171 & 0.842191 & 0.320391 & $0.943502 E-01$ & $-0.177524 E-02$ \\
\hline 181 & 0.887848 & 0.367023 & 0.132015 & $0.129271 E-01$ \\
\hline 191 & 0.933486 & 0.414711 & 0.170507 & $0.285568 \mathrm{E}-01$ \\
\hline 201 & 0.979118 & 0.462746 & 0.209258 & $0.444972 E-01$ \\
\hline
\end{tabular}

First Derivative of Modeshape

$\begin{array}{rllll}1 & 0.249957 E-01 & 0.000000 & -0.644979 E-02 & 0.000000 \\ 11 & 0.249564 E-01 & 0.560284 E-03 & -0.614713 E-02 & -0.466659 E-03 \\ 21 & 0.249195 E-01 & 0.133761 E-02 & -0.613075 E-02 & -0.858673 E-03 \\ 31 & 0.248804 E-01 & 0.218301 E-02 & -0.609334 E-02 & -0.129346 E-02 \\ 41 & 0.248359 E-01 & 0.304435 E-02 & -0.581596 E-02 & -0.178889 E-02 \\ 51 & 0.247841 E-01 & 0.393412 E-02 & -0.515822 E-02 & -0.229707 E-02 \\ 61 & 0.247245 E-01 & 0.490171 E-02 & -0.404835 E-02 & -0.273349 E-02 \\ 71 & 0.246576 E-01 & 0.601012 E-02 & -0.247334 E-02 & -0.300163 E-02 \\ 81 & 0.245847 E-01 & 0.731711 E-02 & -0.469084 E-03 & -0.301281 E-02 \\ 91 & 0.245076 E-01 & 0.886087 E-02 & 0.188952 E-02 & -0.270159 E-02 \\ 101 & 0.244289 E-01 & 0.106501 E-01 & 0.449843 E-02 & -0.203648 E-02 \\ 111 & 0.243511 E-01 & 0.126585 E-01 & 0.723435 E-02 & -0.102597 E-02 \\ 121 & 0.242770 E-01 & 0.148238 E-01 & 0.996460 E-02 & 0.279918 E-03 \\ 131 & 0.242092 E-01 & 0.170511 E-01 & 0.125570 E-01 & 0.179279 E-02 \\ 141 & 0.241500 E-01 & 0.192212 E-01 & 0.148897 E-01 & 0.339361 E-02 \\ 151 & 0.241012 E-01 & 0.212022 E-01 & 0.168611 E-01 & 0.494507 E-02 \\ 161 & 0.240640 E-01 & 0.228670 E-01 & 0.183998 E-01 & 0.630857 E-02 \\ 171 & 0.240384 E-01 & 0.241143 E-01 & 0.194743 E-01 & 0.736578 E-02 \\ 181 & 0.240236 E-01 & 0.248943 E-01 & 0.201031 E-01 & 0.804484 E-02 \\ 191 & 0.240176 E-01 & 0.252389 E-01 & 0.203642 E-01 & 0.835122 E-02 \\ 201 & 0.240166 E-01 & 0.252963 E-01 & 0.204055 E-01 & 0.840306 E-02\end{array}$

Second Derivative of Modeshape

$\begin{array}{rrrrr}1 & 0.000000 & 0.195118 E-03 & 0.301220 E-03 & -0.302912 E-03 \\ 11 & 0.000000 & 0.370241 E-03 & 0.528635 E-04 & -0.210331 E-03 \\ 21 & 0.000000 & 0.435249 E-03 & -0.875183 E-05 & -0.212279 E-03 \\ 31 & 0.000000 & 0.450356 E-03 & 0.672519 E-04 & -0.246663 E-03 \\ 41 & 0.000000 & 0.457658 E-03 & 0.236950 E-03 & -0.270386 E-03 \\ 51 & 0.000000 & 0.483482 E-03 & 0.461618 E-03 & -0.256911 E-03 \\ 61 & 0.000000 & 0.540726 E-03 & 0.707726 E-03 & -0.193842 E-03 \\ 71 & 0.000000 & 0.631208 E-03 & 0.946945 E-03 & -0.804900 E-04 \\ 81 & 0.000000 & 0.748010 E-03 & 0.115614 E-02 & 0.745562 E-04\end{array}$




$\begin{array}{rrrrr}91 & 0.000000 & 0.877819 E-03 & 0.131739 E-02 & 0.255854 E-03 \\ 101 & 0.000000 & 0.100328 E-02 & 0.141794 E-02 & 0.443537 E-03 \\ 111 & 0.000000 & 0.110532 E-02 & 0.145026 E-02 & 0.615745 E-03 \\ 121 & 0.000000 & 0.116554 E-02 & 0.141201 E-02 & 0.751052 E-03 \\ 131 & 0.000000 & 0.116849 E-02 & 0.130605 E-02 & 0.830892 E-03 \\ 141 & 0.000000 & 0.110407 E-02 & 0.114043 E-02 & 0.841988 E-03 \\ 151 & 0.000000 & 0.969868 E-03 & 0.928408 E-03 & 0.778785 E-03 \\ 161 & 0.000000 & 0.773475 E-03 & 0.688436 E-03 & 0.645870 E-03 \\ 171 & 0.000000 & 0.534854 E-03 & 0.444163 E-03 & 0.460409 E-03 \\ 181 & 0.000000 & 0.288682 E-03 & 0.224436 E-03 & 0.254567 E-03 \\ 191 & 0.000000 & 0.866866 E-04 & 0.633004 E-04 & 0.779450 E-04 \\ 201 & 0.000000 & 0.247582 E-13 & 0.170329 E-13 & 0.226193 E-13\end{array}$


TURBN.DAT

DATA INPUT FOR THE EXAMPLE TWO BLADED, DOWNWIND

80 FT DIAM. TEETERING ROTOR

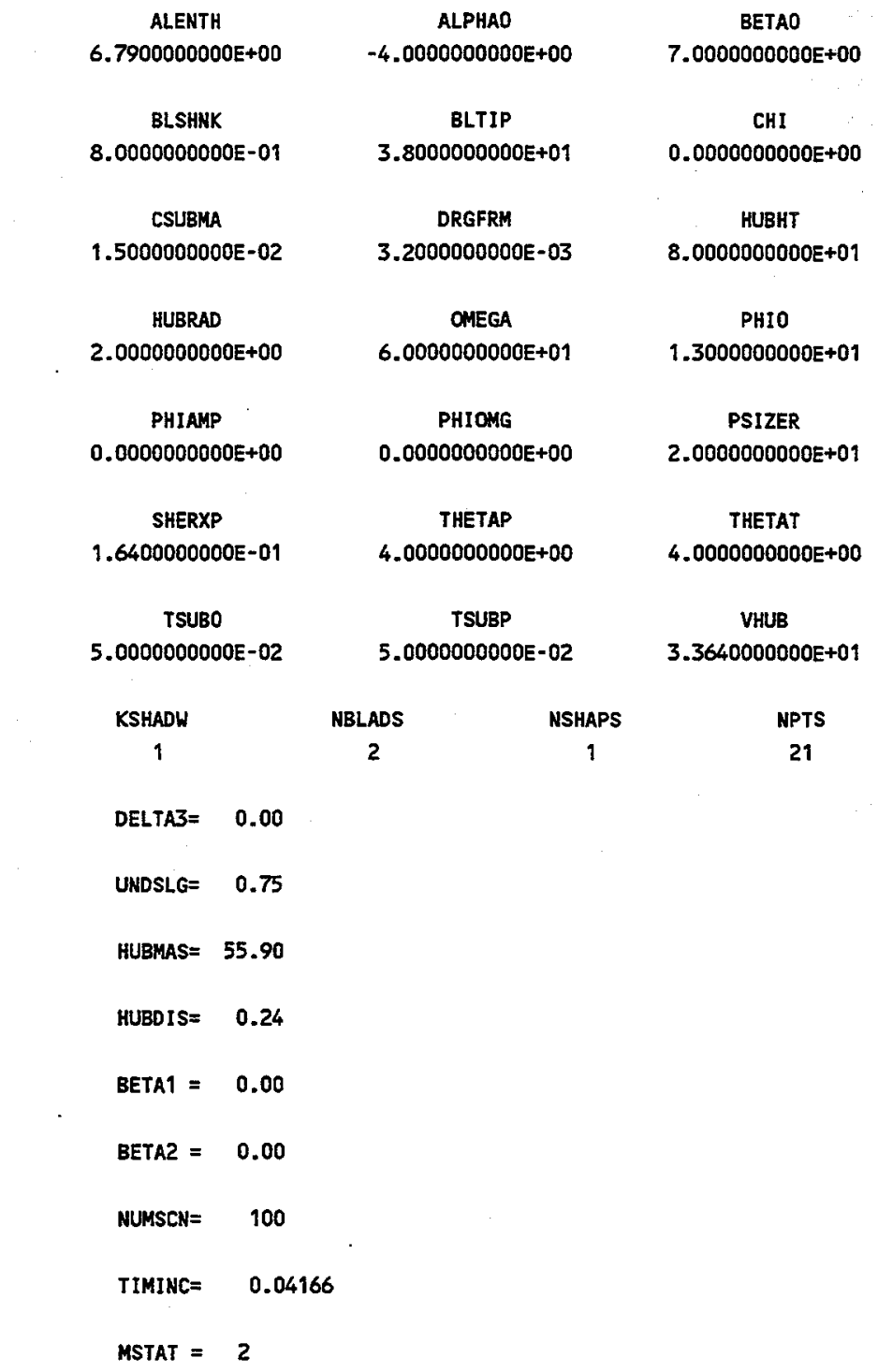

STACI)-

2.000E+01 4.000E+01

CLALFA-

\begin{tabular}{|c|c|c|c|}
\hline $0.0000000 E+00$ & $4.6100000 E+00$ & $4.7366670 E+00$ & 4. $7783330 E+00$ \\
\hline $4.8100000 E+00$ & $4.8416670 E+00$ & $4.8675000 E+00$ & $4.8912500 E+00$ \\
\hline $4.9150000 E+00$ & $4.9387500 E+00$ & $4.9500000 E+00$ & $4.9500000 E+00$ \\
\hline $5.0000000 E+00$ & $5.1187500 E+00$ & $5.1988000 E+00$ & $5.1950000 E+00$ \\
\hline $5.1912000 E+00$ & $5.1874000 E+00$ & $5.1836000 E+00$ & $5.1800000 E+00$ \\
\hline
\end{tabular}

CLMaX - 


$\begin{array}{llll}0.0000000 E+00 & 1.0300000 E+00 & 1.0933330 E+00 & 1.1283330 E+00 \\ 1.1600000 E+00 & 1.1916670 E+00 & 1.2175000 E+00 & 1.2412500 E+00 \\ 1.2650000 E+00 & 1.2887500 E+00 & 1.3500000 E+00 & 1.4450000 E+00 \\ 1.5200000 E+00 & 1.5675000 E+00 & 1.6000000 E+00 & 1.6000000 E+00 \\ 1.6000000 E+00 & 1.6000000 E+00 & 1.6000000 E+00 & 1.6000000 E+00\end{array}$

CDZERO-

$\begin{array}{llll}0.0000000 E+00 & 8.3000000 E-03 & 8.3000000 E-03 & 8.3000000 E-03 \\ 8.3000000 E-03 & 8.3000000 E-03 & 8.3000000 E-03 & 8.3000000 E-03 \\ 8.3000000 E-03 & 8.3000000 E-03 & 8.3000000 E-03 & 8.3000000-03 \\ 8.3000000 E-03 & 8.3000000 E-03 & 8.3000000 E-03 & 8.3000000 E-03 \\ 8.3000000 E-03 & 8.3000000 E-03 & 8.3000000 E-03 & 8.3000000-03\end{array}$

CHORD-

$1.0000000 \mathrm{E}+00$

2. $1580000 E+00$

$2.3163330 E+00$

$2.4981830 E+00$

2. $6828000 E+00$

2.8674160E+00

$2.8369000 E+00$

$2.7295500 E+00$

2.6222010E+00

$2.5148510 E+00$

$2.4075010 E+00$

2.3001500E +00

2. $1928000 \mathrm{E}+00$

2.0854490E+00

$1.9848790 E+00$

$1.8989990 E+00$

$1.8131190 E+00$

$1.7272390 E+00$

$1.6413590 E+00$

$1.5545000 \mathrm{E}+00$

ECNTFN-

$0.0000000 E+00$

$0.0000000 E+00$

$0.0000000 E+00$

$0.0000000 E+00$

$0.0000000 E+00$

$0.0000000 E+00$

$0.0000000 E+00$

$0.0000000 E+00$

$0.0000000 E+00$

$0.0000000 E+00$

$0.0000000 E+00$

$0.0000000 E+00$

$0.0000000 E+00$

$0.0000000 E+00$

$0.0000000 E+00$

$0.0000000 E+00$

$0.0000000 E+00$

$0.0000000 E+00$

$0.0000000 E+00$

$0.0000000 E+00$

ESUBAC-

$0.0000000 E+00$

$0.0000000 E+00$

$0.0000000 E+00$

$0.0000000 E+00$

$0.0000000 E+00$

$0.0000000 E+00$

$0.0000000 E+00$

$0.0000000 E+00$

$0.0000000 E+00$

$0.0000000 E+00$

$0.0000000 E+00$

$0.0000000 E+00$

$0.0000000 E+00$

$0.0000000 E+00$

$0.0000000 E+00$

$0.0000000 E+00$

$0.0000000 E+00$

$0.0000000 E+00$

$0.0000000 E+00$

THETAO-

1.3962630E-01

7.0336770E-02

7.1442140E-02

7.2053010E-02

7.2605690E-02

7.3158380 E-02

7.5136420E-02

7.7623500E-02

8.0110590E-02

8.2597690E-02

8.5521120E-02

8.8837260E-02

9.3200590E-02

1.0496420E-01

1.1126480E-01

1.1756550E-01

$1.2386610=-01$

$1.3016670 \mathrm{E}-01$

1.3700830 E-01

$1.3962630 \mathrm{E}-01$

CKBEND-

$0.0000000 E+00$

$0.0000000 E+00$

$0.0000000 E+00$

$0.0000000 E+00$

$0.0000000 E+00$

$1.0083390 E+02$

$0.0000000 E+00$

$-4.0322250 E+00$

$0.0000000 E+00$

$0.0000000 E+00$

$1.2041450 E+02$

$0.0000000 E+00$

$0.0000000 E+00 \quad-4.0322250 E+00$

$0.0000000 E+00$

$2.8433650 E+01$

CKTOMG-

5.1083540E+00 $0.0000000 E+00$

$0.0000000 E+00 \quad 9.4809340 E-01$

$1.1354120 E-01$

$0.0000000 E+00$

$1.1354120 \mathrm{E}-01$

$0.0000000 E+00$

$0.0000000 E+00$

$1.0213810 \mathrm{E}-01$

$0.0000000 E+00$

$1.0213810 \mathrm{E}-01$

$.2326250 \mathrm{E}-01$

$0.0000000 \mathrm{E}+00$

$0.0000000 E+00 \quad 7.1425090 E-02$

CKTGRV- 


\author{
$0.0000000 E+00$ \\ 5. 1426520E-02 \\ $0.0000000 E+00$ \\ $-7.1287890 \mathrm{E}-03$
}

CKQLOD-

$0.0000000 E+00$

$0.0000000 E+00$

$0.0000000 \mathrm{E}+00$

$0.0000000 E+00$

CMMASS-

$5.6169610 E+00$

$0.0000000 E+00$

$-3.9048480 E-07$

$0.0000000 E+00$

5. 1426520E-02

$0.0000000 E+00$

1.4469950E-02

$0.0000000 E+00$

$0.0000000 E+00$

$0.0000000 E+00$

$0.0000000 \mathrm{E}+00$

$0.0000000 E+00$

$0.0000000 E+00$

5.7911190E-01

$0.0000000 E+00$

4. $1807690 \mathrm{E}-07$

$\operatorname{CKTCRL}(1, K, L)-$

$0.0000000 E+00$

$0.0000000 E+00$

$0.0000000 E+00$

$0.0000000 E+00$

$0.0000000 E+00$

$0.0000000 E+00$

$0.0000000 E+00$

$0.0000000 E+00$

CKTCRL $(2, K, L)-$

3.6609590E-02

$0.0000000 E+00$

$3.9628800 E-03$

$0.0000000 E+00$

$0.0000000 E+00$

$9.3012170 E-03$

$0.0000000 E+00$

$1.3157830 E-03$

CKTCRL $(3, K, L)-$

$0.0000000 E+00$

3.4063490E-03

$0.0000000 E+00$

3.4063490E-03

$0.0000000 E+00$

2.4253440E-03

8.4122110E-04

$0.0000000 E+00$

CKTCRL $(4, K, L)-$

$-2.2678710 E-03$

$0.0000000 E+00$

$7.6378460 \mathrm{E}-04$

$0.0000000 E+00$

$0.0000000 E+00$

2.8308130E-04

$0.0000000 E+00$

$1.4827730 \mathrm{E}-04$

CMRIED-

$0.0000000 E+00$

$6.4966190 E+01$

$0.0000000 E+00$

$-4.2173530 E+00$

CMRGD1-

2. $2779000 E+02$

$0.0000000 E+00$

2.3999150E-01

$0.0000000 E+00$

CHBLNC-

$0.0000000 E+00$

$0.0000000 E+00$

$0.0000000 E+00$

$0.0000000 E+00$

CHGRAV-

$0.0000000 E+00$

$2.1128080 \mathrm{E}+00$

$0.0000000 E+00$

$-2.8395860 E-01$

CMGRV1 -

1.0081580E+01

$0.0000000 E+00$

$-8.0574100 E-01$

$0.0000000 E+00$

CNHUB 1-

$\begin{array}{lll}9.0235100 E-01 \quad 0.0000000 E+00 & 0.0000000 E+00 & 0.0000000 E+00\end{array}$

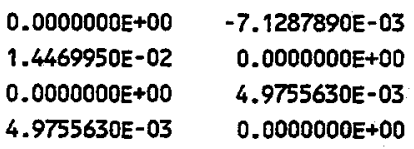

$0.0000000 E+00$

$0.0000000 E+00$

$0.0000000 E+00$

4.1807690E-07

$1.2199980 E-02$

$0.0000000 E+00$

$0.0000000 E+00$

$0.0000000 E+00$

$0.0000000 E+00$

$6.7067570 E-04$

8.4122110E-04

$0.0000000 E+00$

5.1626760E-04

$0.0000000 E+00$

1.4827730E-04

$0000000 E+00$

$1.5749480 E-05$

.

$0.0000000 E+00$

KOSTIF- 

$0.0000000 E+00$
$0.0000000 E+00$
$0.0000000 E+00$
$0.0000000 E+00$

K1STIF-
$0.0000000 E+00$
$0.0000000 E+00$
$0.0000000 E+00$
$0.0000000 E+00$

K2STIF -

\begin{tabular}{llll}
$0.0000000 E+00$ & $0.0000000 E+00$ & $0.0000000 E+00$ & $0.0000000 E+00$ \\
\hline $0.0000000 E+00$ & $0.0000000 E+00$ & $0.0000000 E+00$ & $0.0000000 E+00$ \\
& & & \\
& & & \\
$1.0081500 E+01$ & $9.8663750 E+00$ & $9.5392470 E+00$ & $9.1238200 E+00$ \\
$8.6388350 E+00$ & $8.1051190 E+00$ & $7.5394350 E+00$ & $6.9500780 E+00$ \\
$6.3529620 E+00$ & $5.7644290 E+00$ & $5.1990590 E+00$ & $4.6630750 E+00$ \\
$4.1681130 E+00$ & $3.7192550 E+00$ & $3.3237990 E+00$ & $2.9484800 E+00$ \\
$2.5839090 E+00$ & $2.2341860 E+00$ & $1.9033940 E+00$ & $1.5639160 E+00$ \\
$0.0000000 E+00$ & & &
\end{tabular}

$\operatorname{TCORLS}(2,1)-$

2.1128270E+00

2.1123900E+00

2. 1090430E+00

2. $0997130 \mathrm{E}+00$

2. $0540900 E+00$

2. $0154400 E+00$

$1.7474600 \mathrm{E}+00$

$1.3524730 \mathrm{E}+00$

$1.9650790 \mathrm{E}+00$

$1.6558360 \mathrm{E}+00$

$1.8301280 E+00$
$1.4553350 E+00$

8.6397670E-01

$1.2420960 E+00$

$1.5577070 E+00$

9.9525940E-01

7.1791740E-01

$0.0000000 E+00$

TCORLS(3,1)-

-8.0570250E-01

$-4.4274570 E-01$

8.8817760E-02

4.2563970E-01

4.4329790E-01

$0.0000000 E+00$

-7.5057150E-01

$-6.6788760 \mathrm{E}-01$

$-1.7522270 E-01$

-5.6368380E-01

$-3.1122080 E-01$

2.9907120E-01

$-3.9570120 E-02$

2.0339000E-01

4.6731410E-01

3.7364430E-01

4.5608210E-01

3.6778800E-01

4.6262870E-01

4.1091280E-01

3.1100700E-01

$\operatorname{TCORLS}(4,1)-$

$-2.8395640 \mathrm{E}-01$

$-2.6258720 \mathrm{E}-01$

$-1.5697290 E-01$

-7.8960160 E-03

6.6237290E-02

$-2.8348530 E-01$

$-2.8069860 \mathrm{E}-01$

$-2.2179010 E-01$

$-2.4528320 \mathrm{E}-01$

$-7.9414780 \mathrm{E}-02$

$-2.7412010 \mathrm{E}-01$

-1.1860560 E-01

2.0126780E-02

4.1191160E-02

$-1.9201140 E-01$

$-4.1653340 \mathrm{E}-02$

6.9901270E-02

6.7864180 E- 02

5.6578030 E-02

$0.0000000 E+00$

TGRAV-

$2.6713120 E+01$
$1.6357350 E+01$

$2.3788780 E+01$

2. 1080080E+01

5.9955730E-02

$9.3644120 E+00$

5. 0908870E+00

$1.4324270 E+01$

$1.2497370 E+01$

$1.8609220 E+01$

$8.0567000 E+00$

$6.9181700 E+00$

1.0844640E+01

$4.3842720 \mathrm{E}+00$

3.8037450E +00

5.9310100E+00

$3.2878390 E+00$

$2.3893230 \mathrm{E}+00$

2. $0067140 E+00$

$1.6345970 E+00$

$0.0000000 E+00$

TOMGA-

4.0749440E+02

$3.9888640 \mathrm{E}+02$

$3.8578960 \mathrm{E}+02$

$3.6914690 \mathrm{E}+02$

$3.4970350 E+02$

$2.5785960 E+02$

$1.6969250 \mathrm{E}+02$

$1.0542580 \mathrm{E}+02$

$3.2828990 \mathrm{E}+02$

$2.3415240 \mathrm{E}+02$

$1.5151820 E+02$

$3.0557420 E+02$

$2.8188530 E+02$

$2.1135070 E+02 \quad 1.8970650 E+02$

$1.3548440 E+02 \quad 1.2024650 E+02$

9.1191170E+01

$7.7711350 E+01$

$6.3862130 E+01$

$\operatorname{CIFMOM}(1,1)-$ 


$\begin{array}{llll}0.0000000 E+00 & 0.0000000 E+00 & 0.0000000 E+00 & 0.0000000 E+00 \\ 0.0000000 E+00 & 0.000000 E+00 & 0.0000000 E+00 & 0.000000 E+00 \\ 0.0000000 E+00 & 0.0000000 E+00 & 0.0000000 E+00 & 0.000000 E+00 \\ 0.0000000 E+00 & 0.000000 E+00 & 0.0000000 E+00 & 0.000000 E+00 \\ 0.0000000 E+00 & 0.0000000 E+00 & 0.0000000 E+00 & 0.000000 E+00\end{array}$

CIFMOM(2, I)-

$0.0000000 E+00$

$0.0000000 E+00$

$0.0000000 E+00 \quad 0.0000000 E+00$

$0.0000000 E+00$

$0.0000000 E+00$

$0.0000000 E+00$

$0.0000000 E+00$

$0.0000000 E+00$

$0.0000000 E+00$

$0.0000000 E+00$

$0.0000000 E+00$

$0.0000000 E+00$

$0.0000000 E+00$

$0.0000000 E+00$

$0.0000000 E+00$

$0.0000000 E+00$

$0.0000000 E+00$

$0.0000000 E+00$

$0.0000000 E+00$

CIFMOM( 3,1$)$ -

$0.0000000 E+00$

$0.0000000 E+00$

$0.0000000 E+00$

$0.0000000 E+00$

$0.0000000 E+00$

$0.0000000 E+00$

$0.0000000 E+00$

$0.0000000 E+00$

$0.0000000 E+00$

$0.0000000 E+00$

$0.0000000 E+00$

$0.0000000 E+00$

$0.0000000 E+00$

$0.0000000 E+00$

$0.0000000 E+00$

$0.0000000 E+00$

$0.0000000 E+00$

$0.0000000 E+00$

$0.0000000 E+00$

$0.0000000 E+00$

$0.0000000 E+00$

CI FMOM( 4,1$)$ -

$0.0000000 E+00$

$0.0000000 E+00$

$0.0000000 E+00$

$0.0000000 E+00$

$0.0000000 E+00$

$0.0000000 E+00$

$0.0000000 E+00$

$0.0000000 E+00$

$0.0000000 E+00$

$0.0000000 E+00$

$0.0000000 E+00$

$0.0000000 E+00$

$0.0000000 E+00$

$0.0000000 E+00$

$0.0000000 E+00$

$0.0000000 E+00$

$0.0000000 E+00$

$0.0000000 E+00$

DELTIM-

$\begin{array}{llll}0.000000 E+00 & 0.0000000 E+00 & 0.0000000 E+00 & 0.0000000 E+00 \\ 0.000000 E+00 & 0.0000000 E+00 & 0.0000000 E+00 & 0.0000000 E+00 \\ 0.0000000 E+00 & 0.0000000 E+00 & 0.0000000 E+00 & 0.0000000 E+00 \\ 0.000000 E+00 & 0.0000000 E+00 & 0.0000000 E+00 & 0.0000000 E+00 \\ 0.000000 E+00 & 0.0000000 E+00 & 0.0000000 E+00 & 0.0000000 E+00\end{array}$

OFFMAS-

$\begin{array}{llll}0.0000000 E+00 & 0.0000000 E+00 & 0.000000 E+00 & 0.0000000 E+00 \\ 0.0000000 E+00 & 0.0000000 E+00 & 0.000000 E+00 & 0.0000000 E+00 \\ 0.0000000 E+00 & 0.0000000 E+00 & 0.000000 E+00 & 0.0000000 E+00 \\ 0.0000000 E+00 & 0.000000 E+00 & 0.000000 E+00 & 0.0000000 E+00 \\ 0.0000000 E+00 & 0.0000000 E+00 & 0.000000 E+00 & 0.000000 E+00\end{array}$

$0.0000000 E+00$
$0.499915 E-01$
$\begin{array}{ll}-0.128996 E-01 & 0.000000\end{array}$
$0.974451 E-01$
$0.479990 E-03 \quad-0.247922 E-01 \quad-0.470760 E-03$
0.144827
$0.226373 E-02 \quad-0.364379 E-01 \quad-0.172893 E-02$
0.192138
$0.560398 E-02 \quad-0.480739 E-01 \quad-0.376290 E-02$
0.239369
0.105679E-01 -0.594390E-01 -0.668386E-02
0.286509
0.171897E-01 $-0.699322 E-01 \quad-0.105695 E-01$
0.333544
$0.255666 \mathrm{E}-01 \quad-0.787526 \mathrm{E}-01 \quad-0.153675 \mathrm{E}-01$
0.380458
0.359055E-01 $-0.850203 E-01 \quad-0.208500 E-01$
0.427239
$0.485312 E-01 \quad-0.878786 E-01 \quad-0.266104 E-01$
0.473877
$0.638611 E-01 \quad-0.865778 E-01 \quad-0.320938 E-01$
0.520367
0.823587E-01 -0.805395E-01 -0.366515E-01
0.566707
0.104471
$-0.694031 E-01 \quad-0.396127 E-01$
0.612903
0.130561
$-0.530526 E-01 \quad-0.403623 E-01$
0.658964
0.160841
$-0.316252 E-01 \quad-0.384173 E-01$ 


\begin{tabular}{|c|c|c|c|}
\hline 0.704903 & 0.195319 & $-0.550104 E-02$ & $-0.334936 E-01$ \\
\hline 0.750740 & 0.233762 & $0.247261 E-01$ & $-0.255528 E-01$ \\
\hline 0.796495 & 0.275687 & $0.582962 E-01$ & $-0.148218 E-01$ \\
\hline 0.842191 & 0.320391 & $0.943502 E-01$ & $-0.177524 E-02$ \\
\hline 0.887848 & 0.367023 & 0.132015 & 0.129271E-01 \\
\hline 0.933486 & 0.414711 & 0.170507 & $0.2855688-01$ \\
\hline 0.979118 & 0.462746 & 0.209258 & $0.444972 E-01$ \\
\hline $0.249957 E-01$ & 0.000000 & $-0.644979 E-02$ & 0.000000 \\
\hline $0.249564 E-01$ & $0.560284 E-03$ & $-0.614713 E-02$ & $-0.466659 \mathrm{E}-03$ \\
\hline $0.249195 E-01$ & $0.133761 E-02$ & $-0.613075 E-02$ & $-0.858673 E-03$ \\
\hline $0.248804 E-01$ & $0.218301 E-02$ & $-0.609334 E-02$ & $-0.129346 E-02$ \\
\hline $0.248359 E-01$ & $0.304435 E-02$ & $-0.581596 E-02$ & $-0.178889 E-02$ \\
\hline $0.247841 E-01$ & $0.393412 E-02$ & $-0.515822 E-02$ & $-0.229707 E-02$ \\
\hline $0.247245 E-01$ & $0.490171 E-02$ & $-0.404835 E-02$ & $-0.273349 E-02$ \\
\hline $0.246576 E-01$ & $0.601012 E-02$ & $-0.247334 E-02$ & $-0.300163 E-02$ \\
\hline $0.245847 E-01$ & $0.731711 E-02$ & $-0.469084 E-03$ & $-0.301281 E-02$ \\
\hline $0.245076 E-01$ & $0.886087 E-02$ & $0.188952 E-02$ & $-0.270159 E-02$ \\
\hline $0.2442899-01$ & $0.106501 E-01$ & $0.449843 E-02$ & $-0.203648 E-02$ \\
\hline $0.243511 E-01$ & $0.126585 E-01$ & $0.723435 \mathrm{E}-02$ & $-0.102597 E-02$ \\
\hline $0.242770 E-01$ & $0.148238 \mathrm{E}-01$ & $0.996460 \mathrm{E}-02$ & $0.279918 E-03$ \\
\hline $0.242092 E-01$ & $0.170511 E-01$ & $0.125570 E-01$ & 0.179279E-02 \\
\hline $0.241500 E-01$ & $0.192212 E-01$ & $0.148897 E-01$ & $0.339361 E-02$ \\
\hline $0.241012 E-01$ & $0.212022 E-01$ & $0.168611 E-01$ & $0.494507 E-02$ \\
\hline $0.240640 E-01$ & $0.228670 E-01$ & $0.183998 E-01$ & $0.630857 E-02$ \\
\hline $0.240384 E-01$ & $0.241143 E-01$ & $0.194743 E-01$ & $0.736578 E-02$ \\
\hline $0.240236 E-01$ & $0.248943 E-01$ & $0.201031 E-01$ & $0.804484 E-02$ \\
\hline $0.240176 \mathrm{E}-01$ & $0.252389 E-01$ & $0.203642 E-01$ & $0.835122 E-02$ \\
\hline $0.240166 E-01$ & $0.252963 E-01$ & $0.204055 E-01$ & $0.840306 E-02$ \\
\hline 0.000000 & $0.195118 E-03$ & $0.301220 E-03$ & $-0.302912 E-03$ \\
\hline 0.000000 & $0.370241 E-03$ & $0.528635 E-04$ & $-0.210331 E-03$ \\
\hline 0.000000 & $0.435249 \mathrm{E}-03$ & $-0.875183 E-05$ & $-0.212279 E-03$ \\
\hline 0.000000 & $0.450356 \mathrm{E}-03$ & $0.672519 E-04$ & $-0.246663 \mathrm{E}-03$ \\
\hline 0.000000 & $0.457658 E-03$ & $0.236950 \mathrm{E}-03$ & $-0.270386 E-03$ \\
\hline 0.000000 & $0.483482 E-03$ & $0.461618 E-03$ & $-0.256911 E-03$ \\
\hline 0.000000 & $0.540726 E-03$ & $0.707726 E-03$ & $-0.193842 E-03$ \\
\hline 0.000000 & $0.631208 E-03$ & $0.946945 E-03$ & $-0.804900 E-04$ \\
\hline 0.000000 & $0.748010 \mathrm{E}-03$ & $0.115614 E-02$ & $0.745562 E-04$ \\
\hline 0.000000 & $0.877819 E-03$ & $0.131739 E-02$ & $0.255854 E-03$ \\
\hline 0.000000 & $0.100328 E-02$ & $0.141794 E-02$ & $0.443537 E-03$ \\
\hline 0.000000 & $0.110532 E-02$ & $0.145026 \mathrm{E}-02$ & $0.615745 E-03$ \\
\hline 0.000000 & $0.116554 E-02$ & $0.141201 E-02$ & $0.751052 E-03$ \\
\hline 0.000000 & $0.116849 \mathrm{E}-02$ & $0.130605 \mathrm{E}-02$ & $0.830892 E-03$ \\
\hline 0.000000 & $0.110407 E-02$ & $0.114043 E-02$ & $0.841988 E-03$ \\
\hline 0.000000 & $0.969868 E-03$ & $0.928408 E-03$ & $0.778785 E-03$ \\
\hline 0.000000 & $0.773475 E-03$ & $0.688436 E-03$ & $0.645870 E-03$ \\
\hline 0.000000 & $0.534854 E-03$ & $0.444163 E-03$ & $0.460409 E-03$ \\
\hline 0.000000 & $0.288682 E-03$ & $0.224436 E-03$ & $0.254567 E-03$ \\
\hline 0.000000 & $0.866866 E-04$ & $0.633004 E-04$ & $0.779450 \mathrm{E}-04$ \\
\hline 0.000000 & $0.247582 E-13$ & $0.170329 E-13$ & $0.226193 E-13$ \\
\hline
\end{tabular}


Appendix B

Module 2 Interactive Run Listing and Output Files 
Program For Analysis of Horizontal Axis Wind Turbine

Response To Dynamic Loads

MODULE 2

Operations Menu

(R)ead in a data file

(S)et up and run the model

(D)iagnostic run

(T)urbulence run

(Q)uit

Enter Option $(R, S, D, T, Q)>$

$\mathbf{R}$

Enter the name of the file that contains run-data > RUNB.DAT

Reading in new data... done.

Analyzing a teetering hub.

Operations Menu

-

(R)ead in a data file

(S)et up and run the model

(D)iagnostic run

(T)urbulence run

(Q)uit

Enter Option $(R, S, D, T, Q)>$

S

Current values for the 'free' variables:

$\begin{array}{rlrlrl}1 & \text { ALENTH }= & 6.7900 \text { feet } & 12 & \text { PHIAMP }= & 0.0000 \text { degrees } \\ 2 & \text { ALPHAO }= & -4.0000 \text { degrees } & 13 \text { PHIOMG }= & 0.0000 \text { degrees/sec } \\ 3 \text { BETAO }= & 7.0000 \text { degrees } & 14 \text { PSISHD }= & 180.0000 \text { degrees } \\ 4 \text { CHI }= & 0.0000 \text { degrees } & 15 \text { PSIZER }= & 20.0000 \text { degrees } \\ 5 & \text { GRAV }= & 32.1740 \text { feet/sec^2 } & 16 \text { RHOAIR }= & 0.0020 \text { slugs/feet^3 } \\ 6 \text { HUBHT }= & 80.0000 \text { feet } & 17 \text { SHERXP }= & 0.1640 \\ 7 \text { KSHADW }= & 1 & 18 \text { THETAP }= & 2.0000 \text { degrees } \\ 8 \text { NBLADS }= & 2 & 19 \text { THETAT }= & 4.0000 \text { degrees } \\ 9 \text { NSHAPS }= & 1 & 20 \text { TSUBO } & 0.0500 \\ 10 \text { OMEGA }= & 60.0000 \text { RPM } & 21 \text { TSUBP }= & 0.0500 \\ 11 \text { PHIO }= & 13.0000 \text { degrees } & 22 \text { VHUB } & 33.6400 \text { feet/second } \\ 23 \text { BETA1 }= & 0.0000 \text { degrees } & 24 \text { BETA2 }= & 0.0000 \text { degrees } \\ 25 & \text { TIMINC }= & 0.041660 \text { sec } & 26 \text { NUMSCN }= & 100\end{array}$

Would you like to change any values? $(Y,=N)>$

$y$

Enter the number of the variable you would like

to change $(1-26)>$

9

Enter nEw INTEGER value for NSHAPS >

2 
Current values for the 'free' variables:

\begin{tabular}{|c|c|c|c|c|c|}
\hline ALENTH & 6.7900 & feet & 12 & PHIAMP = & 0.0000 degrees \\
\hline ALPHAO & -4.0000 & degrees & 13 & PHIOMG = & 0.0000 degrees $/ \mathrm{sec}$ \\
\hline BETAO & 7.0000 & degrees & 14 & PSISHD $=$ & 180.0000 degrees \\
\hline CHI & 0.0000 & degrees & 15 & PSIZER = & 20.0000 degrees \\
\hline GRAV & 32.1740 & feet $/ \sec ^{\wedge} 2$ & 16 & RHOAIR = & 0.0020 slugs/feet^ \\
\hline HUBHT & 80.0000 & feet & 17 & SHERXP $=$ & 0.1640 \\
\hline KSHADW & $=$ & & 18 & THETAP $=$ & 2.0000 degrees \\
\hline NBLADS & $=$ & & 19 & THETAT $=$ & 4.0000 degrees \\
\hline NSHAPS & $=$ & & 20 & TSUBO $=$ & 0.0500 \\
\hline OMEGA & 60.0000 & RPM & 21 & TSUBP & 0.0500 \\
\hline PHIO & 13.0000 & degrees & 22 & VHUB & 33.6400 feet/second \\
\hline BETA1 & 0.0000 & degrees & 24 & BETA2 & 0.0000 degrees \\
\hline TIMIN & 0.041660 & sec & 26 & NUMSCN $=$ & 100 \\
\hline
\end{tabular}

Would you like to change any values? $\left(Y_{,}=N\right)>$

N

The current values of the run parameters are:

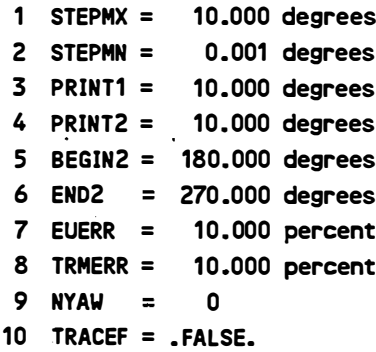

Maximum Step Size Minimum Step size Printout Interval in Region 1 Printout Interval in Region 2 Beginning of Print Region 2 End of Print Region 2 Max value of Euler error function Convergence Criterion for Trim Solution No. of Disk Revolutions for Yawing Soln. Trace Flag

Do you want to change any of these values? $\left(Y_{,}=N\right)>$

Y

Enter number of variable you wish to change >

1

Enter new REAL value for STEPMX >

$\mathbf{5 . 0 0 0 0 0}$

The current values of the run parameters are:

$\begin{array}{rlr}1 & \text { STEPMX }= & 5.000 \text { degrees } \\ 2 & \text { STEPMN }= & 0.001 \text { degrees } \\ 3 & \text { PRINT1 }= & 10.000 \text { degrees } \\ 4 & \text { PRINT2 }= & 10.000 \text { degrees } \\ 5 & \text { BEGIN2 }= & 180.000 \text { degrees } \\ 6 & \text { END2 }= & 270.000 \text { degrees } \\ 7 & \text { EUERR }= & 10.000 \text { percent } \\ 8 & \text { TRMERR }= & 10.000 \text { percent } \\ 9 & \text { NYAW }= & 0 \\ 10 & \text { TRACEF }=. \text { FALSE. }\end{array}$
Maximu Step size

Minimun Step size

Printout Interval in Region 1

Printout Interval in Region 2

Beginning of Print Region 2

End of Print Region 2

Max value of Euler error function

Convergence Criterion for Trim Solution

No. of Disk Revolutions for Yawing Soln.

Trace Flag

Do you want to change any of these values? $(Y,=N)>$

$\mathbf{Y}$

Enter number of variable you wish to change > 
Enter new REAL value for EUERR >

5.00000

The current values of the run parameters are:
$i$ STEPMX $=5.000$ degrees
Maximum Step Size
2 STEPMN $=0.001$ degrees
Minimu Step Size
3 PRINT1 $=10.000$ degrees
Printout Interval in Region 1
4 PRINT2 $=10.000$ degrees
5 BEGIN2 $=180.000$ degrees
Printout Interval in Region 2
6 END2 $=270.000$ degrees
Beginning of Print Region 2
7 EUERR $=5.000$ percent
End of Print Region 2
8 TRMERR $=10.000$ percent
Max value of Euler error function
9 NYAW $=0$
Convergence Criterion for Trim Solution
TRACEF $=$.FALSE.
No. of Disk Revolutions for Yawing Soln.
Trace Flag

Do you want to change any of these values? $(Y,=N)>$

$Y$

Enter number of variable you wish to change >

Enter new REAL value for TRMERR >

5.00000

The current values of the run parameters are:
1 STEPMX $=5.000$ degrees
Maximu Step Size
2 STEPMN $=0.001$ degrees Minimm Step Size
3 PRINTI $=10.000$ degrees Printout Interval in Region 1
4 PRINT2 $=10.000$ degrees Printout Interval in Region 2
5 BEGIN2 $=180.000$ degrees Beginning of Print Region 2
6 END2 $=270.000$ degrees End of Print Region 2
7 EUERR $=\quad 5.000$ percent Max value of Euler error function
8 TRMERR $=5.000$ percent Convergence Criterion for Trim Solution
9 NYAW $=0 \quad$ No. of Disk Revolutions for Yawing Soln.
10 TRACEF = .FALSE. Trace Flag

Do you want to change any of these values? $(Y,=N)>$

N

Product of CMMASS premultiplied by its inverse:
$1.0000000 \quad 0.0000000$
$0.0000000 \quad 1.0000000$

Are you ready to run the model? $(=Y, N)>$

Y

Enter name of results file [=RESULTS.DAT] >

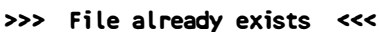

Do you want to overwrite the old data? $(Y, N)>$

$Y$

Do you want to input your own values for the initial modal deflections, or do you want

SUBROUTINE STRTUP to calculate values for you? 
If you want to input your own then input a "Y" or.a "y", If you want SUB STRTUP to do it

input an "N" or an "n" >

$Y$

Read in SO(1) and SO(2)<

(all must be nonzero)

$-0.100000$

1.00000

Trim test \#01 completed. The trim condition was not satisfied.

Trim test \#02 completed. The trim condition was not satisfied.

Trim test \#03 completed. The trim condition was satisfied.

Do you want to perform fourier analysis of

any of the results data? <

$Y$

Read in the result data item \#

that you want analyzed <

$1=$ flapwise displacement

$2=$ flapwise slope

$3=$ flapwise segment velocity

4 = blade tension

5 = blade edgewise shear

6 = btade flapwise shear

7 = blade flapwise moment

8 = blade edgewise moment

$9=$ blade torsional moment

7

Read in the highest order harmonic desired <

The highest order possible is 10P <

10

Do you want to perform fourier analysis of

any of the results data? <

N

Read in name of shaft loads output file <

SHAFTB.DAT

Do you want to do another run with this data? $(Y,=N)>$

N

Operations Menu

-.................

(R)ead in a data file

(S)et. $u$ and run the model

(D)iagnostic run

(T)urbulence run

(Q)uit

Enter Option $(R, S, D, T, Q)>$

T

Turbulence Analysis Run Set-up

Read in the nare of the wind residual time series file WINO-2B.DAT 
Is the wind data in metric units (meters/sec)?

$\mathbf{Y}$

Read in the name of the file for loads output turblodb.dat

Operations Menu

-

(R)ead in a data file

(S)et up and run the model

(D)iagnostic run

(T)urbulence run

(Q)uit

Enter Option $(R, S, D, T, Q)>$

N

Invalid response. Please try again.

Enter Option $(R, S, D, T, Q)>$

Q

STRAP terminated normally. 
Analysis of Wind Turbine Blade Loads National Renewable Energy Laboratory
TURBN.DAT

DATA INPUT FOR THE EXAMPLE TWO BLADED, DOWNWIND

80 FT DIAM. TEETERING ROTOR

Teetering Rotor

Run Parameters, blade data and machine data used in this analysis:

HUBRAD $=2.000$ feet
OMEGA $=60.000$ RPM
PHIAMP $=0.000$ degrees
PHIOMG $=0.000$ degrees $/ \mathrm{sec}$
PHIO $=13.000$ degrees
PSIZER $=20.000$ degrees
RHOAIR $=0.002$ slugs $/ \mathrm{ft} \wedge 3$
SHERXP $=0.164$ degrees
STEPMX $=5.000$ degrees
STEPMN $=\quad 0.001$ degrees

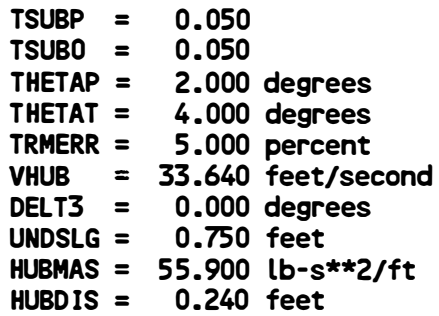

KSHADW $=1$ NBLADS $=2$ NSHAPS $=2$ NYAW $=0$ 


$\begin{array}{cc}\text { PSI }=\text { ( DEG. }) \text { Teeter Damper Loads ( } f t-\text { lb) } \\ 0 & 0.000000 \\ 10 & 0.000000 \\ 20 & 0.000000 \\ 30 & 0.000000 \\ 40 & 0.000000 \\ 50 & 0.000000 \\ 60 & 0.000000 \\ 70 & 0.000000 \\ 80 & 0.000000 \\ 90 & 0.000000 \\ 100 & 0.000000 \\ 110 & 0.000000 \\ 120 & 0.000000 \\ 130 & 0.000000 \\ 140 & 0.000000 \\ 150 & 0.000000 \\ 160 & 0.000000 \\ 170 & 0.000000 \\ 180 & 0.000000 \\ 190 & 0.000000 \\ 200 & 0.000000 \\ 210 & 0.000000 \\ 220 & 0.000000 \\ 230 & 0.000000 \\ 240 & 0.000000 \\ 250 & 0.000000 \\ 260 & 0.000000 \\ 270 & 0.000000 \\ 280 & 0.000000 \\ 290 & 0.000000 \\ 300 & 0.000000 \\ 310 & 0.000000 \\ 320 & 0.000000 \\ 330 & 0.000000 \\ 340 & 0.000000 \\ 350 & 0.000000 \\ 360 & 0.000000 \\ & \end{array}$

B-8 


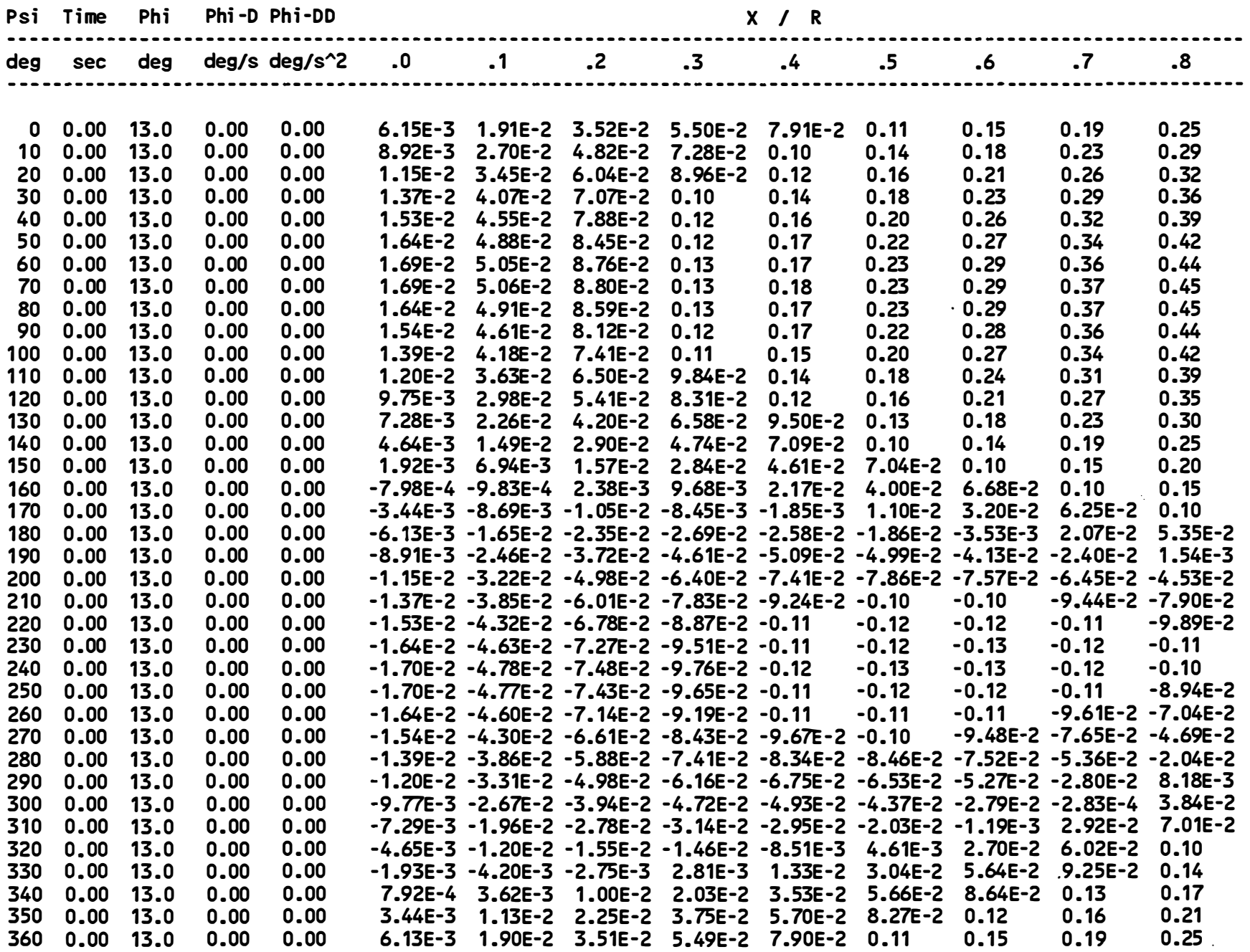




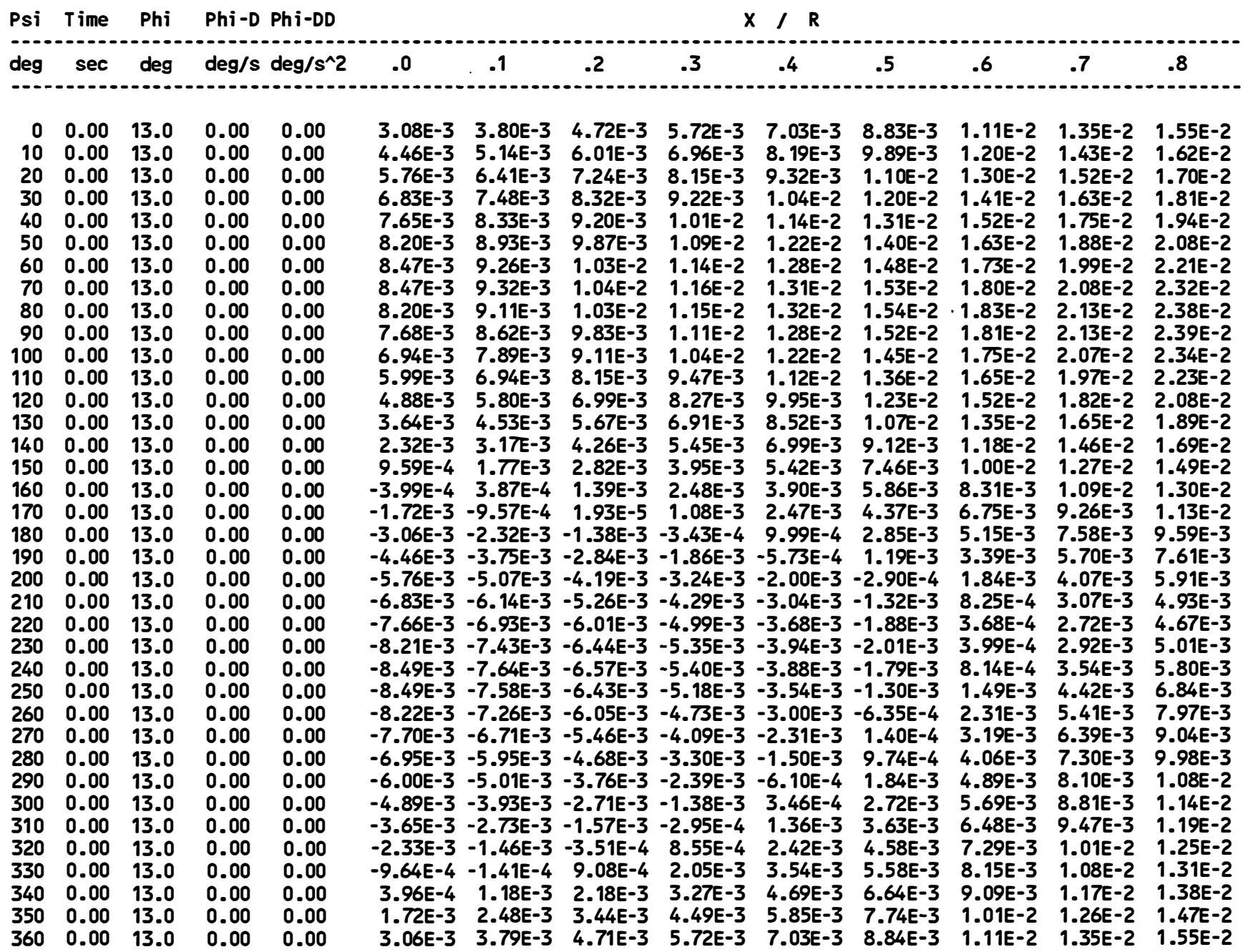




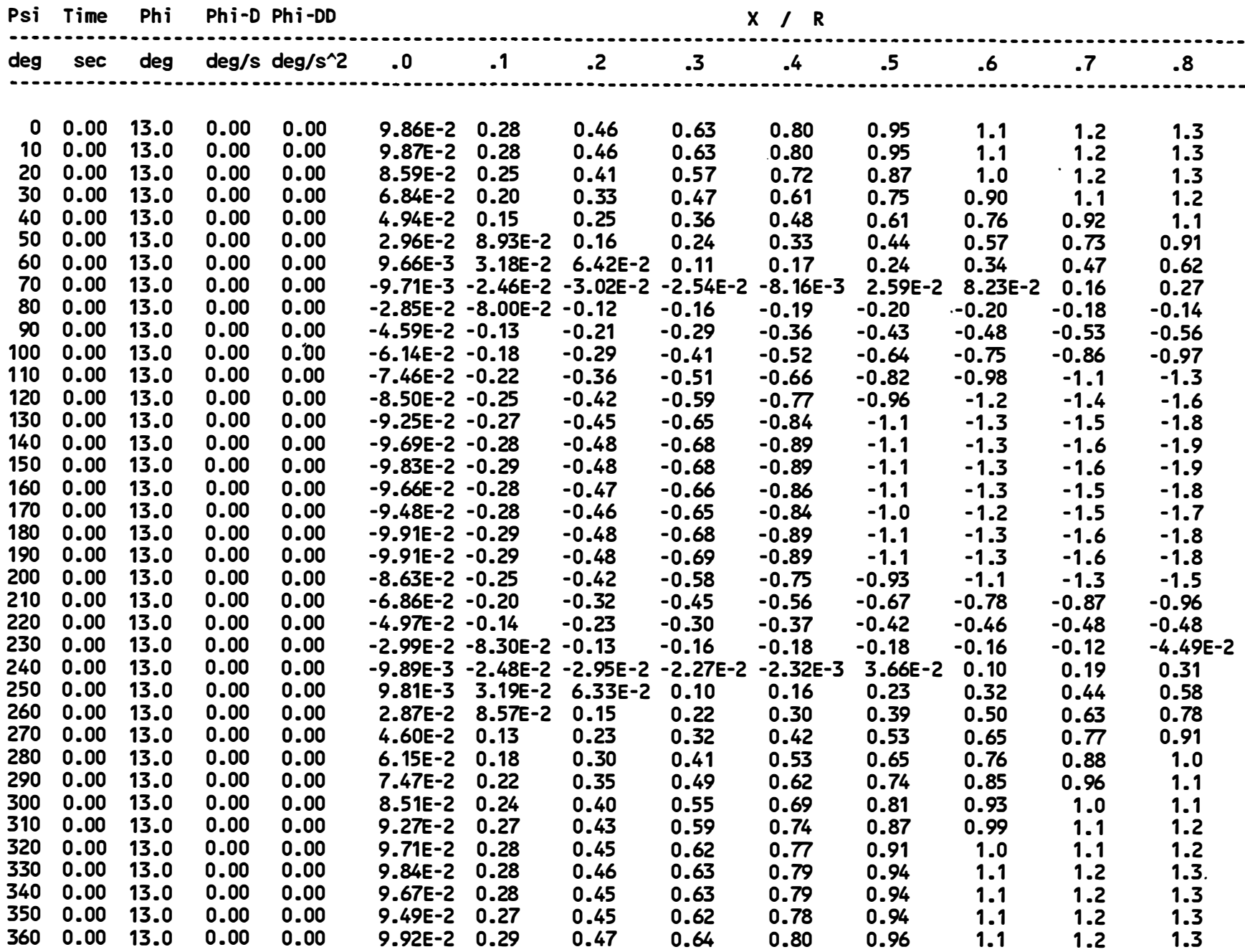




\begin{tabular}{|c|c|c|c|c|c|c|c|c|c|c|c|c|c|}
\hline eg & sec & deg & deg/ & $g / s^{\wedge} 2$ & .0 & .1 & .2 & .3 & .4 & .5 & .6 & .7 & .8 \\
\hline $\begin{array}{r}0 \\
10 \\
20 \\
30 \\
40 \\
50 \\
60 \\
70 \\
80 \\
90 \\
100 \\
110 \\
120 \\
130 \\
140 \\
150 \\
160 \\
170 \\
180 \\
190 \\
200 \\
210 \\
220 \\
230 \\
240 \\
250 \\
260 \\
270 \\
280 \\
290 \\
300 \\
310 \\
320 \\
330 \\
340 \\
350\end{array}$ & $\begin{array}{l}0.00 \\
0.00 \\
0.00 \\
0.00 \\
0.00 \\
0.00 \\
0.00 \\
0.00 \\
0.00 \\
0.00 \\
0.00 \\
0.00 \\
0.00 \\
0.00 \\
0.00 \\
0.00 \\
0.00 \\
0.00 \\
0.00 \\
0.00 \\
0.00 \\
0.00 \\
0.00 \\
0.00 \\
0.00 \\
0.00 \\
0.00 \\
0.00 \\
0.00 \\
0.00 \\
0.00 \\
0.00 \\
0.00 \\
0.00 \\
0.00 \\
0.00 \\
0.00\end{array}$ & $\begin{array}{l}13.0 \\
13.0 \\
13.0 \\
13.0 \\
13.0 \\
13.0 \\
13.0 \\
13.0 \\
13.0 \\
13.0 \\
13.0 \\
13.0 \\
13.0 \\
13.0 \\
13.0 \\
13.0 \\
13.0 \\
13.0 \\
13.0 \\
13.0 \\
13.0 \\
13.0 \\
13.0 \\
13.0 \\
13.0 \\
13.0 \\
13.0 \\
13.0 \\
13.0 \\
13.0 \\
13.0 \\
13.0 \\
13.0 \\
13.0 \\
13.0 \\
13.0 \\
13.0\end{array}$ & $\begin{array}{l}0.00 \\
0.00 \\
0.00 \\
0.00 \\
0.00 \\
0.00 \\
0.00 \\
0.00 \\
0.00 \\
0.00 \\
0.00 \\
0.00 \\
0.00 \\
0.00 \\
0.00 \\
0.00 \\
0.00 \\
0.00 \\
0.00 \\
0.00 \\
0.00 \\
0.00 \\
0.00 \\
0.00 \\
0.00 \\
0.00 \\
0.00 \\
0.00 \\
0.00 \\
0.00 \\
0.00 \\
0.00 \\
0.00 \\
0.00 \\
0.00 \\
0.00 \\
0.00\end{array}$ & $\begin{array}{l}0.00 \\
0.00 \\
0.00 \\
0.00 \\
0.00 \\
0.00 \\
0.00 \\
0.00 \\
0.00 \\
0.00 \\
0.00 \\
0.00 \\
0.00 \\
0.00 \\
0.00 \\
0.00 \\
0.00 \\
0.00 \\
0.00 \\
0.00 \\
0.00 \\
0.00 \\
0.00 \\
0.00 \\
0.00 \\
0.00 \\
0.00 \\
0.00 \\
0.00 \\
0.00 \\
0.00 \\
0.00 \\
0.00 \\
0.00 \\
0.00 \\
0.00 \\
0.00\end{array}$ & $\begin{array}{l}1.52 E+4 \\
1.52 E+4 \\
1.53 E+4 \\
1.53 E+4 \\
1.54 E+4 \\
1.55 E+4 \\
1.57 E+4 \\
1.58 E+4 \\
1.59 E+4 \\
1.61 E+4 \\
1.62 E+4 \\
1.64 E+4 \\
1.65 E+4 \\
1.66 E+4 \\
1.67 E+4 \\
1.68 E+4 \\
1.69 E+4 \\
1.69 E+4 \\
1.69 E+4 \\
1.69 E+4 \\
1.69 E+4 \\
1.68 E+4 \\
1.67 E+4 \\
1.66 E+4 \\
1.65 E+4 \\
1.64 E+4 \\
1.62 E+4 \\
1.61 E+4 \\
1.59 E+4 \\
1.58 E+4 \\
1.57 E+4 \\
1.55 E+4 \\
1.54 E+4 \\
1.54 E+4 \\
1.53 E+4 \\
1.52 E+4\end{array}$ & $\begin{array}{l}1.46 \mathrm{E}+4 \\
1.46 \mathrm{E}+4 \\
1.46 \mathrm{E}+4 \\
1.46 \mathrm{E}+4 \\
1.47 \mathrm{E}+4 \\
1.48 \mathrm{E}+4 \\
1.49 \mathrm{E}+4 \\
1.50 \mathrm{E}+4 \\
1.51 \mathrm{E}+4 \\
1.52 \mathrm{E}+4 \\
1.53 \mathrm{E}+4 \\
1.55 \mathrm{E}+4 \\
1.56 \mathrm{E}+4 \\
1.57 \mathrm{E}+4 \\
1.57 \mathrm{E}+4 \\
1.58 \mathrm{E}+4 \\
1.59 \mathrm{E}+4 \\
1.59 \mathrm{E}+4 \\
1.59 \mathrm{E}+4 \\
1.59 \mathrm{E}+4 \\
1.59 \mathrm{E}+4 \\
1.58 \mathrm{E}+4 \\
1.57 \mathrm{E}+4 \\
1.57 \mathrm{E}+4 \\
1.56 \mathrm{E}+4 \\
1.55 \mathrm{E}+4 \\
1.54 \mathrm{E}+4 \\
1.52 \mathrm{E}+4 \\
1.51 \mathrm{E}+4 \\
1.50 \mathrm{E}+4 \\
1.49 \mathrm{E}+4 \\
1.48 \mathrm{E}+4 \\
1.47 \mathrm{E}+4 \\
1.47 \mathrm{E}+4 \\
1.46 \mathrm{E}+4 \\
1.46 \mathrm{E}+4 \\
1.46 \mathrm{E}+4\end{array}$ & $\begin{array}{l}1.33 E+4 \\
1.33 E+4 \\
1.33 E+4 \\
1.34 E+4 \\
1.34 E+4 \\
1.35 E+4 \\
1.35 E+4 \\
1.36 E+4 \\
1.37 E+4 \\
1.38 E+4 \\
1.39 E+4 \\
1.40 E+4 \\
1.41 E+4 \\
1.41 E+4 \\
1.42 E+4 \\
1.43 E+4 \\
1.43 E+4 \\
1.43 E+4 \\
1.43 E+4 \\
1.43 E+4 \\
1.43 E+4 \\
1.43 E+4 \\
1.42 E+4 \\
1.41 E+4 \\
1.41 E+4 \\
1.40 E+4 \\
1.39 E+4 \\
1.38 E+4 \\
1.37 E+4 \\
1.36 E+4 \\
1.35 E+4 \\
1.35 E+4 \\
1.34 E+4 \\
1.34 E+4 \\
1.33 E+4 \\
1.33 E+4 \\
1.33 E+4\end{array}$ & $\begin{array}{l}1.17 E+4 \\
1.17 E+4 \\
1.17 E+4 \\
1.17 E+4 \\
1.18 E+4 \\
1.18 E+4 \\
1.19 E+4 \\
1.19 E+4 \\
1.20 E+4 \\
1.21 E+4 \\
1.21 E+4 \\
1.22 E+4 \\
1.23 E+4 \\
1.23 E+4 \\
1.24 E+4 \\
1.24 E+4 \\
1.24 E+4 \\
1.25 E+4 \\
1.25 E+4 \\
1.25 E+4 \\
1.24 E+4 \\
1.24 E+4 \\
1.24 E+4 \\
1.23 E+4 \\
1.23 E+4 \\
1.22 E+4 \\
1.21 E+4 \\
1.21 E+4 \\
1.20 E+4 \\
1.19 E+4 \\
1.19 E+4 \\
1.18 E+4 \\
1.18 E+4 \\
1.17 E+4 \\
1.17 E+4 \\
1.17 E+4 \\
1.17 E+4\end{array}$ & $\begin{array}{l}9.88 E+3 \\
9.89 E+3 \\
9.90 E+3 \\
9.92 E+3 \\
9.95 E+3 \\
9.99 E+3 \\
1.00 E+4 \\
1.01 E+4 \\
1.01 E+4 \\
1.02 E+4 \\
1.02 E+4 \\
1.03 E+4 \\
1.03 E+4 \\
1.04 E+4 \\
1.04 E+4 \\
1.04 E+4 \\
1.05 E+4 \\
1.05 E+4 \\
1.05 E+4 \\
1.05 E+4 \\
1.05 E+4 \\
1.04 E+4 \\
1.04 E+4 \\
1.04 E+4 \\
1.03 E+4 \\
1.03 E+4 \\
1.02 E+4 \\
1.02 E+4 \\
1.01 E+4 \\
1.01 E+4 \\
1.00 E+4 \\
9.99 E+3 \\
9.95 E+3 \\
9.92 E+3 \\
9.90 E+3 \\
9.89 E+3 \\
9.88 E+3\end{array}$ & $\begin{array}{l}8.13 E+3 \\
8.13 E+3 \\
8.14 E+3 \\
8.15 E+3 \\
8.18 E+3 \\
8.20 E+3 \\
8.23 E+3 \\
8.27 E+3 \\
8.30 E+3 \\
8.34 E+3 \\
8.38 E+3 \\
8.42 E+3 \\
8.45 E+3 \\
8.48 E+3 \\
8.51 E+3 \\
8.53 E+3 \\
8.55 E+3 \\
8.56 E+3 \\
8.56 E+3 \\
8.56 E+3 \\
8.55 E+3 \\
8.53 E+3 \\
8.51 E+3 \\
8.49 E+3 \\
8.46 E+3 \\
8.42 E+3 \\
8.38 E+3 \\
8.35 E+3 \\
8.31 E+3 \\
8.27 E+3 \\
8.24 E+3 \\
8.20 E+3 \\
8.18 E+3 \\
8.15 E+3 \\
8.14 E+3 \\
8.13 E+3 \\
8.13 E+3\end{array}$ & $\begin{array}{l}6.54 E+3 \\
6.54 E+3 \\
6.55 E+3 \\
6.56 E+3 \\
6.58 E+3 \\
6.60 E+3 \\
6.62 E+3 \\
6.64 E+3 \\
6.67 E+3 \\
6.70 E+3 \\
6.73 E+3 \\
6.75 E+3 \\
6.78 E+3 \\
6.80 E+3 \\
6.82 E+3 \\
6.84 E+3 \\
6.85 E+3 \\
6.86 E+3 \\
6.86 E+3 \\
6.86 E+3 \\
6.85 E+3 \\
6.84 E+3 \\
6.82 E+3 \\
6.80 E+3 \\
6.78 E+3 \\
6.76 E+3 \\
6.73 E+3 \\
6.70 E+3 \\
6.67 E+3 \\
6.65 E+3 \\
6.62 E+3 \\
6.60 E+3 \\
6.58 E+3 \\
6.56 E+3 \\
6.55 E+3 \\
6.54 E+3 \\
6.54 E+3\end{array}$ & $\begin{array}{l}5.23 E+3 \\
5.23 E+3 \\
5.24 E+3 \\
5.25 E+3 \\
5.26 E+3 \\
5.27 E+3 \\
5.29 E+3 \\
5.31 E+3 \\
5.33 E+3 \\
5.35 E+3 \\
5.37 E+3 \\
5.39 E+3 \\
5.41 E+3 \\
5.42 E+3 \\
5.44 E+3 \\
5.45 E+3 \\
5.46 E+3 \\
5.47 E+3 \\
5.47 E+3 \\
5.47 E+3 \\
5.46 E+3 \\
5.45 E+3 \\
5.44 E+3 \\
5.43 E+3 \\
5.41 E+3 \\
5.39 E+3 \\
5.37 E+3 \\
5.35 E+3 \\
5.33 E+3 \\
5.31 E+3 \\
5.29 E+3 \\
5.27 E+3 \\
5.26 E+3 \\
5.21 E+3\end{array}$ & $\begin{array}{l}4.07 \mathrm{E}+3 \\
4.07 \mathrm{E}+3 \\
4.08 \mathrm{E}+3 \\
4.09 \mathrm{E}+3 \\
4.09 \mathrm{E}+3 \\
4.11 \mathrm{E}+3 \\
4.12 \mathrm{E}+3 \\
4.13 \mathrm{E}+3 \\
4.15 \mathrm{E}+3 \\
4.16 \mathrm{E}+3 \\
4.18 \mathrm{E}+3 \\
4.19 \mathrm{E}+3 \\
4.20 \mathrm{E}+3 \\
4.22 \mathrm{E}+3 \\
4.23 \mathrm{E}+3 \\
4.24 \mathrm{E}+3 \\
4.24 \mathrm{E}+3 \\
4.25 \mathrm{E}+3 \\
4.25 \mathrm{E}+3 \\
4.25 \mathrm{E}+3 \\
4.25 \mathrm{E}+3 \\
4.24 \mathrm{E}+3 \\
4.23 \mathrm{E}+3 \\
4.22 \mathrm{E}+3 \\
4.21 \mathrm{E}+3 \\
4.19 \mathrm{E}+3 \\
4.18 \mathrm{E}+3 \\
4.16 \mathrm{E}+3 \\
4.15 \mathrm{E}+3 \\
4.13 \mathrm{E}+3 \\
4.12 \mathrm{E}+3 \\
4.11 \mathrm{E}+3 \\
4.09 \mathrm{E}+3 \\
4.09 \mathrm{E}+3 \\
4.08 \mathrm{E}+3 \\
4.07 \mathrm{E}+3 \\
4.07 \mathrm{E}+3\end{array}$ \\
\hline
\end{tabular}




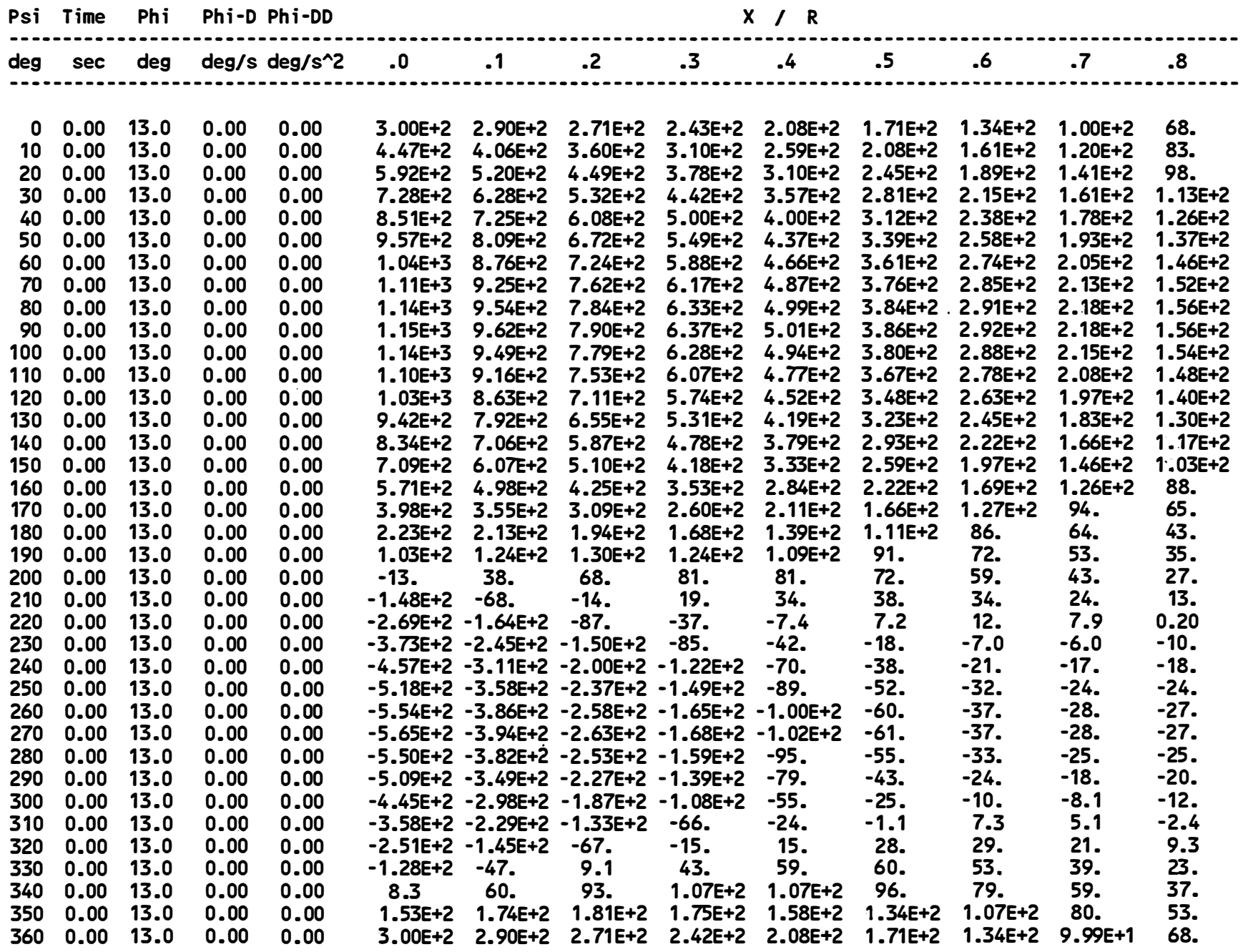




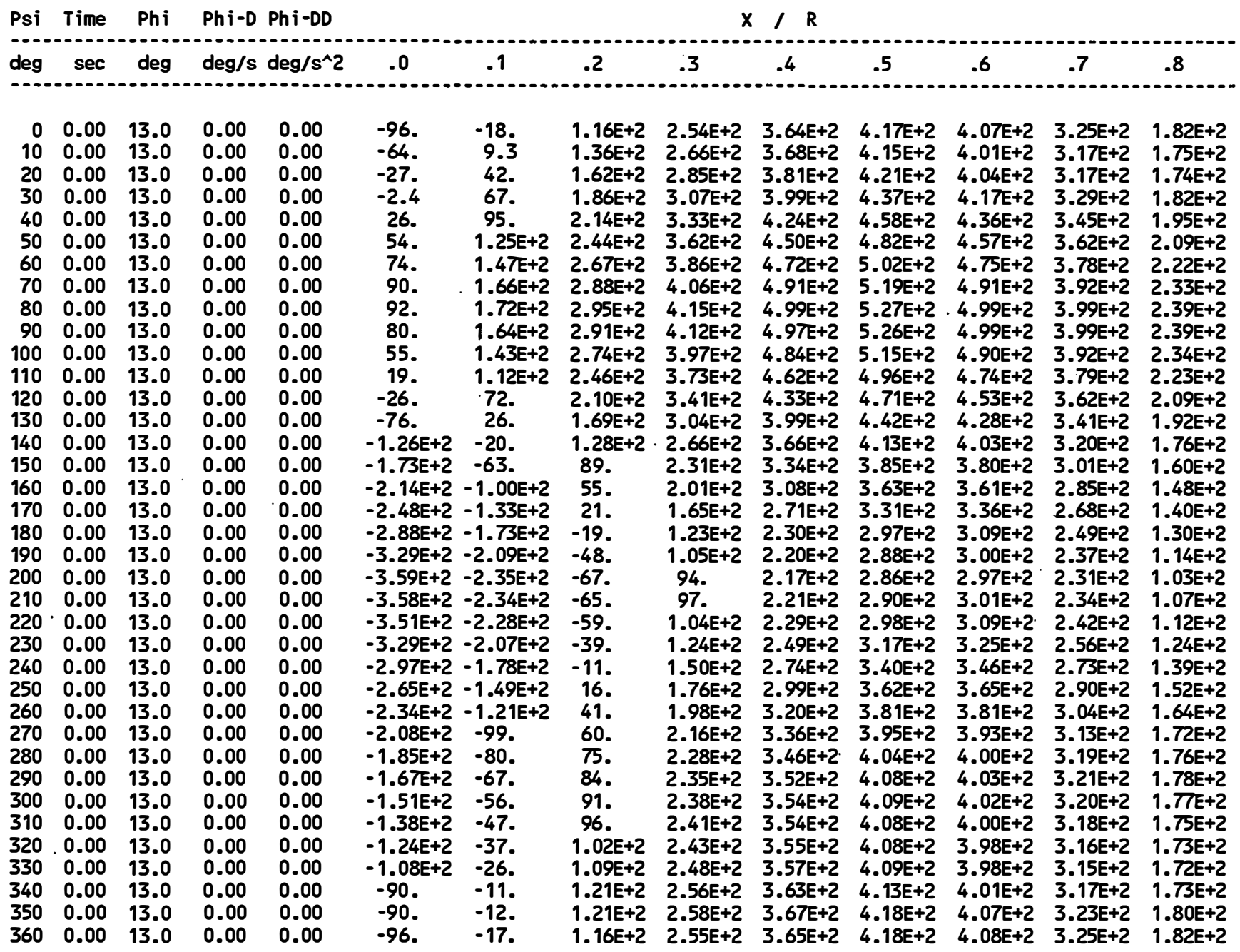




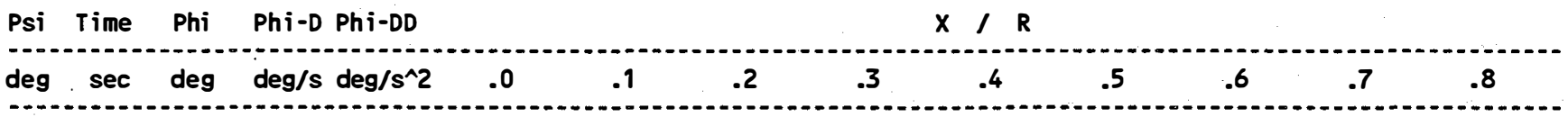

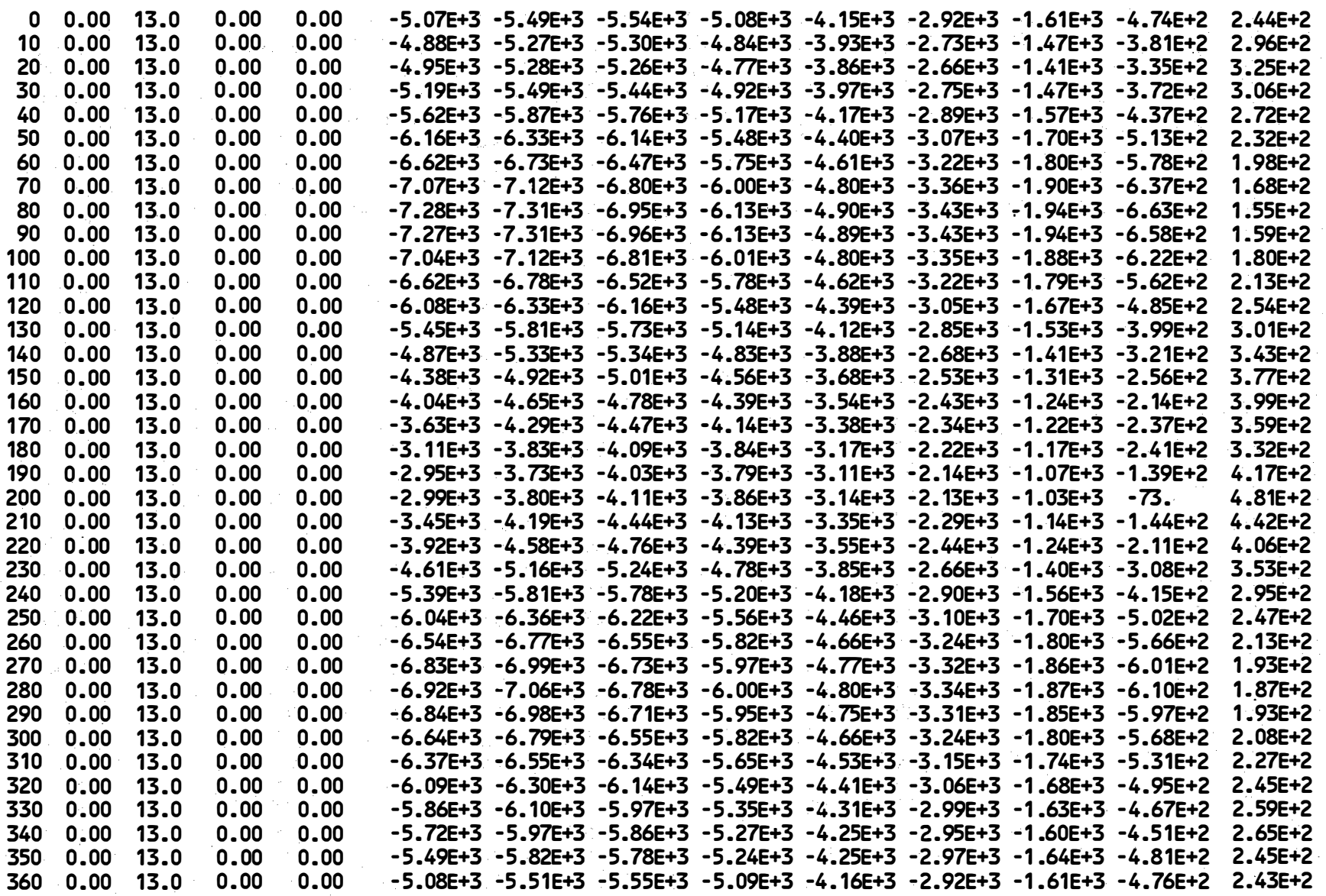

Average Flapwise Moment $=-5764.5$ (ft-Lbs) at the $20 \%$ station 


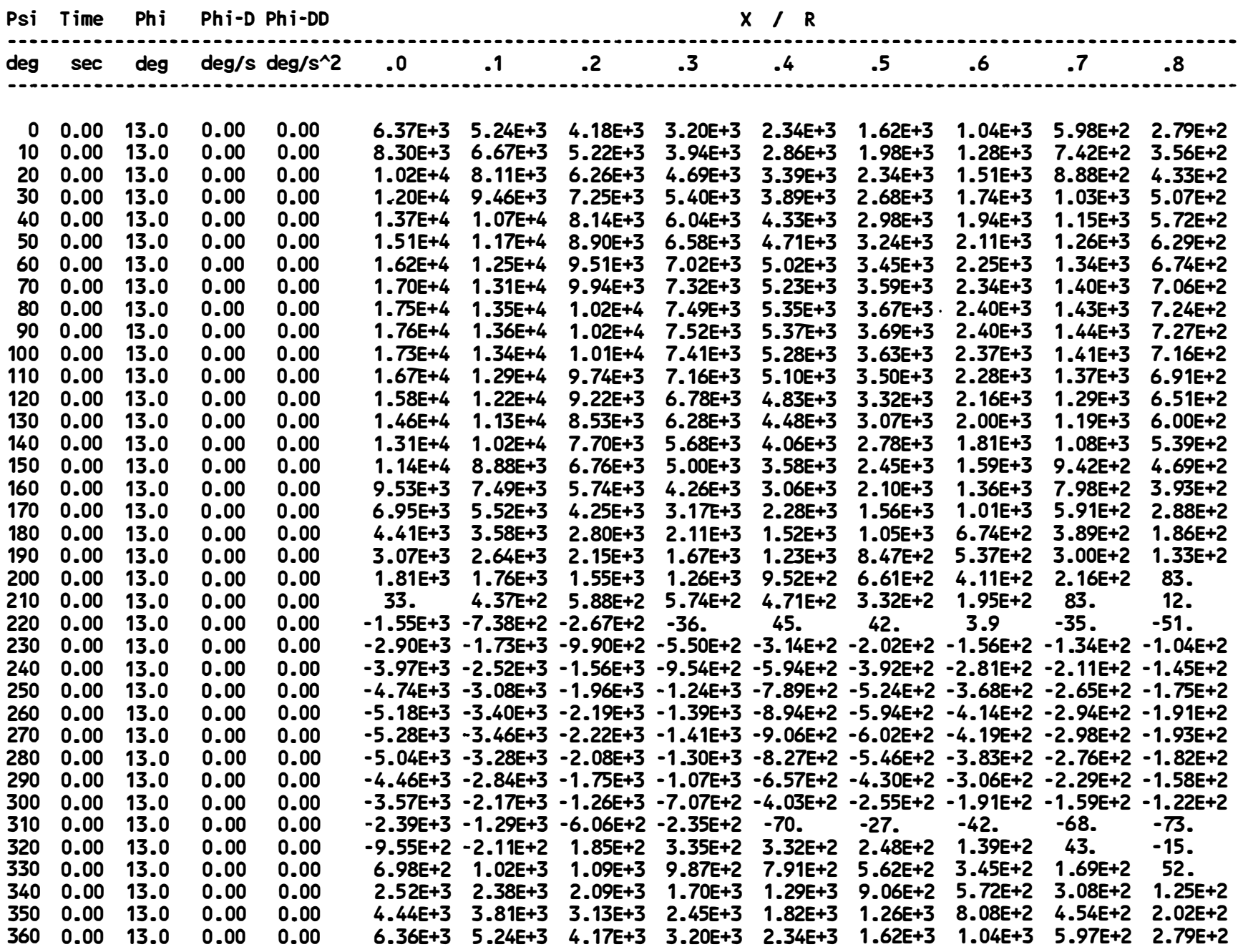




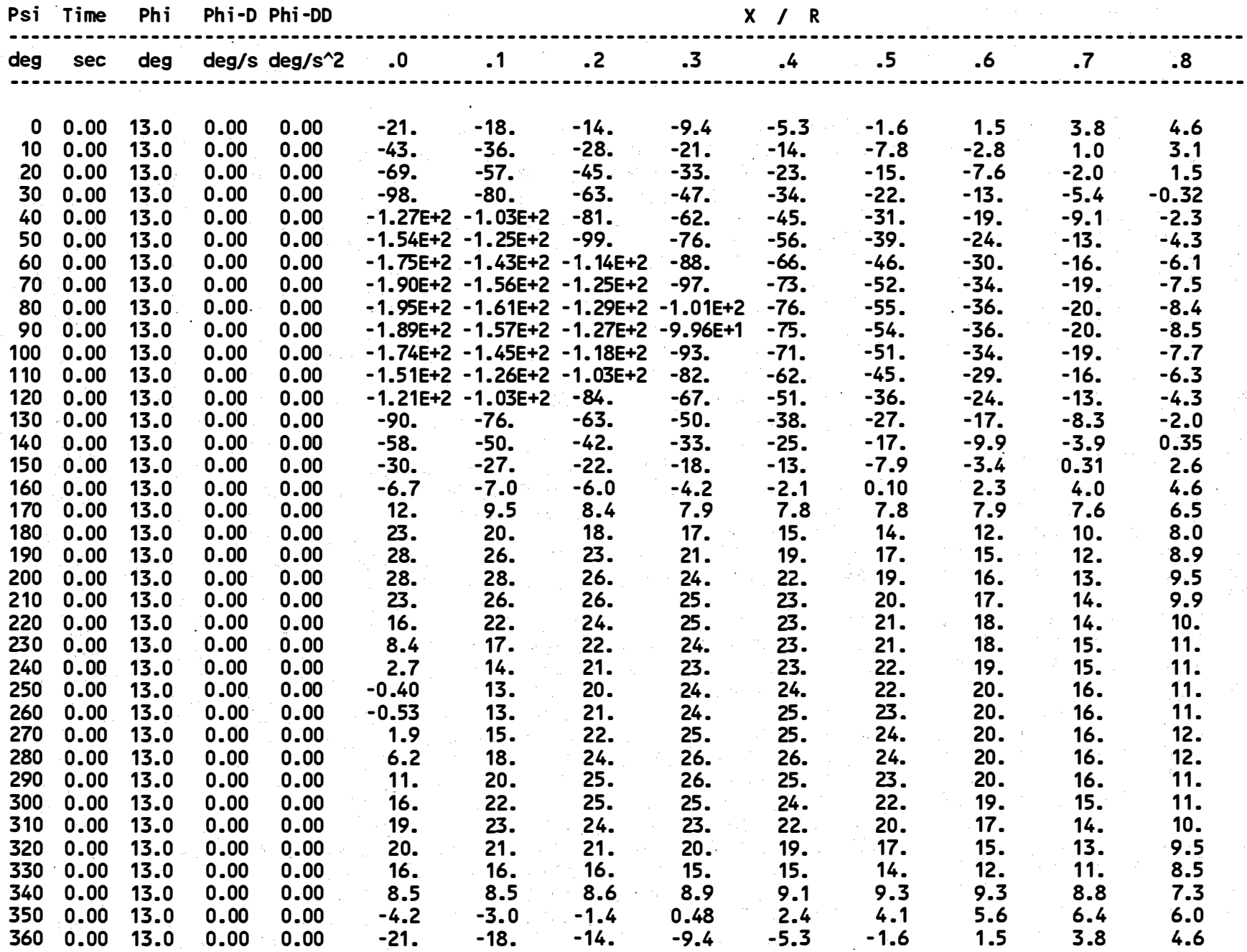


Blade flapwise moment expansion coefficients

$X / R$

\begin{tabular}{|c|c|c|c|c|c|c|c|c|c|c|}
\hline & .0 & 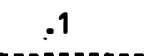 & .2 & .3 & .4 & .5 & .6 & .7 & .8 & .9 \\
\hline $\begin{array}{l}A 0= \\
B O= \\
-\end{array}$ & $\begin{array}{l}-5.50 E+3 \\
0.00\end{array}$ & $\begin{array}{l}-5.84 E+3 \\
0.00\end{array}$ & $\begin{array}{l}-5.76 E+3 \\
0.00\end{array}$ & $\begin{array}{l}-5.19 E+3 \\
0.00\end{array}$ & $\begin{array}{l}-4.17 E+3 \\
0.00\end{array}$ & $\begin{array}{l}-2.90 \mathrm{E}+3 \\
0.00\end{array}$ & $\begin{array}{l}-1.57 E+3 \\
0.00\end{array}$ & $\begin{array}{c}-4.32 E+2 \\
0.00\end{array}$ & $\begin{array}{l}2.77 E+2 \\
0.00\end{array}$ & $\begin{array}{l}3.83 E \\
0.00\end{array}$ \\
\hline $\begin{array}{l}A 1= \\
B 1=\end{array}$ & $\begin{array}{l}-9.53 E+2 \\
-2.04 E+2\end{array}$ & $\begin{array}{l}-7.53 E+2 \\
-1.38 E+2\end{array}$ & $\begin{array}{c}-6.11 E+2 \\
-93 .\end{array}$ & $\begin{array}{l}-5.00 E+2 \\
-64 .\end{array}$ & $\begin{array}{l}-3.95 E+2 \\
-47\end{array}$ & $\begin{array}{l}-2.89 E+2 \\
-39\end{array}$ & $\begin{array}{c}-1.99 E+2 \\
-32 .\end{array}$ & $\begin{array}{l}-1.2 \pi E+2 \\
-24 .\end{array}$ & $\begin{array}{l}-68 . \\
-15 .\end{array}$ & $\begin{array}{l}-24 . \\
-6.0\end{array}$ \\
\hline $\begin{array}{l}A 2= \\
B 2= \\
-\end{array}$ & $\begin{array}{r}1.45 E+3 \\
3.51 E+2 \\
\end{array}$ & $\begin{array}{l}1.23 E+3 \\
2.93 E+2 \\
\end{array}$ & $\begin{array}{l}1.01 E+3 \\
2.38 E+2\end{array}$ & $\begin{array}{l}8.03 E+2 \\
1.87 E+2 \\
\end{array}$ & $\begin{array}{r}6.07 E+2 \\
1.41 E+2 \\
-\end{array}$ & $\begin{array}{r}4.33 E+2 \\
1.01 E+2 \\
\end{array}$ & $\begin{array}{l}2.88 E+2 \\
67 .\end{array}$ & $\begin{array}{c}1.71 E+2 \\
42 .\end{array}$ & $\begin{array}{l}84 . \\
22 .\end{array}$ & $\begin{array}{c}25 . \\
8.1\end{array}$ \\
\hline $\begin{array}{l}A 3= \\
B 3=\end{array}$ & $\begin{array}{r}-17 . \\
18 .\end{array}$ & $\begin{array}{r}-29 . \\
20 .\end{array}$ & $\begin{array}{l}-36 . \\
21 .\end{array}$ & $\begin{array}{l}-36 . \\
21 .\end{array}$ & $\begin{array}{l}-29 . \\
18 .\end{array}$ & $\begin{array}{c}-18 . \\
14 .\end{array}$ & $\begin{array}{r}-7.4 \\
9.0\end{array}$ & $\begin{array}{l}1.4 \\
4.5\end{array}$ & $\begin{array}{l}5.7 \\
1.3\end{array}$ & $\begin{array}{r}4.6 \\
-0.21\end{array}$ \\
\hline $\begin{array}{l}A 4= \\
B 4=\end{array}$ & $\begin{array}{r}-1.08 E+2 \\
1.71 E+2\end{array}$ & $\begin{array}{l}-95 \\
1.47 E+2\end{array}$ & $1.24 E+2$ & $\begin{array}{l}-73 . \\
1.01 E+2\end{array}$ & $\begin{array}{l}-66 . \\
80 .\end{array}$ & $\begin{array}{r}-59 . \\
60 .\end{array}$ & $\begin{array}{r}-51 . \\
43 .\end{array}$ & $\begin{array}{r}-41 . \\
28 .\end{array}$ & $\begin{array}{c}-28 \\
16 .\end{array}$ & $\begin{array}{r}-13 . \\
5.7\end{array}$ \\
\hline $\begin{array}{l}A 5= \\
B 5=\end{array}$ & $\begin{array}{l}1.5 \\
1.7\end{array}$ & $\begin{array}{r}-13 . \\
3.9\end{array}$ & $\begin{array}{c}-22 . \\
5.3\end{array}$ & $\begin{array}{r}-25 . \\
5.7\end{array}$ & $\begin{array}{r}-20 . \\
5.1\end{array}$ & $\begin{array}{r}-12 . \\
3.7\end{array}$ & $\begin{array}{r}-3.5 \\
2.2\end{array}$ & $\begin{array}{l}3.1 \\
0.80\end{array}$ & $\begin{array}{r}6.1 \\
-0.18\end{array}$ & $\begin{array}{r}4.5 \\
-0.41\end{array}$ \\
\hline $\begin{array}{l}A 6= \\
B 6=\end{array}$ & $\begin{array}{l}0.77 \\
55 .\end{array}$ & $\begin{array}{r}-3.3 \\
48 .\end{array}$ & $\begin{array}{r}-7.6 \\
40 .\end{array}$ & $\begin{array}{r}-13 . \\
34 .\end{array}$ & $\begin{array}{r}-19 . \\
27 .\end{array}$ & $\begin{array}{r}-23 . \\
21 .\end{array}$ & $\begin{array}{r}-24 . \\
15 .\end{array}$ & $\begin{array}{r}-23 . \\
9.7 \\
-\end{array}$ & $\begin{array}{r}-17 . \\
5.4 \\
-\end{array}$ & $\begin{array}{r}-8.6 \\
2.0\end{array}$ \\
\hline $\begin{array}{l}A 7= \\
B 7=\end{array}$ & $\begin{array}{r}-6.8 \\
1.3\end{array}$ & $\begin{array}{r}-17 . \\
2.4\end{array}$ & $\begin{array}{l}-24 \\
3.1\end{array}$ & $\begin{array}{r}-24 . \\
3.4\end{array}$ & $\begin{array}{r}-20 \\
3.1\end{array}$ & $\begin{array}{r}-12 \\
2.4\end{array}$ & $\begin{array}{r}-4.6 \\
1.4\end{array}$ & $\begin{array}{l}1.5 \\
0.52\end{array}$ & $\begin{array}{c}4.5 \\
-6.82 E-2\end{array}$ & $\begin{array}{r}3.5 \\
-0.22\end{array}$ \\
\hline $\begin{array}{l}A 8= \\
B 8=\end{array}$ & $\begin{array}{l}20 . \\
36 .\end{array}$ & $\begin{array}{l}14 . \\
31 .\end{array}$ & $\begin{array}{l}7.4 \\
26 .\end{array}$ & $\begin{array}{c}0.88 \\
21 .\end{array}$ & $\begin{array}{c}-6.2 \\
17 .\end{array}$ & $\begin{array}{l}-12 . \\
13 .\end{array}$ & $\begin{array}{r}-15 . \\
9.2\end{array}$ & $\begin{array}{r}-15 . \\
6.0\end{array}$ & $\begin{array}{r}-12 . \\
3.4\end{array}$ & $\begin{array}{r}-6.2 \\
1.2\end{array}$ \\
\hline $\begin{array}{l}A 9= \\
B 9=\end{array}$ & $\begin{array}{l}-3.7 \\
-5.1\end{array}$ & $\begin{array}{l}-12 . \\
-3.5\end{array}$ & $\begin{array}{l}-17 \\
-2.2\end{array}$ & $\begin{array}{l}-17 \\
-1.3\end{array}$ & $\begin{array}{l}-14 \\
-0.81\end{array}$ & $\begin{array}{r}-8.5 \\
-0.60\end{array}$ & $\begin{array}{r}-3.0 \\
-0.54\end{array}$ & $\begin{array}{r}1.4 \\
-0.56\end{array}$ & $\begin{array}{r}3.5 \\
-0.51\end{array}$ & $\begin{array}{r}2.7 \\
-0.30\end{array}$ \\
\hline $\begin{array}{l}A^{*}= \\
B^{*}=\end{array}$ & $\begin{array}{l}12 . \\
15 .\end{array}$ & $\begin{array}{l}7.9 \\
13 .\end{array}$ & $\begin{array}{l}3.9 \\
11 .\end{array}$ & $\begin{array}{r}-0.44 \\
9.1\end{array}$ & $\begin{array}{r}-5.2 \\
7.4\end{array}$ & $\begin{array}{r}-8.9 \\
5.7\end{array}$ & $\begin{array}{r}-11 . \\
4.1\end{array}$ & $\begin{array}{r}-11 . \\
2.7\end{array}$ & $\begin{array}{r}-8.7 \\
1.5\end{array}$ & $\begin{array}{l}-4.4 \\
0.57\end{array}$ \\
\hline
\end{tabular}


Mean Rotor Torque and Power for Trim Solution

$\begin{array}{lc}\text { Mean Rotor Torque }= & 13545.72 \mathrm{ft} \text {-Lbs } \\ \text { Mean Rotor Power }= & 115.39 \mathrm{KH} \\ \text { No. of Data Points }= & 36\end{array}$

No. of Data Points $=$ 36 
THIS IS THE SHAFT LOADS OUTPUT FILE

Low-Speed Shaft Loads in Rotor coordinates

\begin{tabular}{|c|c|c|c|c|c|c|}
\hline Azimuth Angle & Fxr & Fyr & Fzr & Mxr & Myr & Mzr \\
\hline 0.000 & 83.224 & 3521.565 & -1711.795 & -562.079 & 11988.680 & -228.418 \\
\hline 10.000 & 353.294 & 3511.730 & -1693.655 & -568.739 & 12619.370 & -449.934 \\
\hline 20.000 & 615.885 & 3517.800 & -1625.991 & -675.134 & 13332.490 & -667.649 \\
\hline 30.000 & 887.776 & 3543.516 & -1505.106 & -473.615 & 13393.730 & -923.316 \\
\hline 40.000 & 1132.749 & 3578.086 & -1339.407 & -522.801 & 13472.770 & -1152.804 \\
\hline 50.000 & 1342.854 & 3627.853 & -1132.409 & -470.260 & 13569.430 & -1348.364 \\
\hline 60.000 & 1511.618 & 3679.315 & -890.146 & -278.797 & 13666.320 & -1503.510 \\
\hline 70.000 & 1634.316 & 3727.356 & -621.588 & -247.934 & 13748.860 & -1613.187 \\
\hline 80.000 & 1706.979 & 3759.993 & -333.723 & -151.865 & 13804.550 & -1672.482 \\
\hline 90.000 & 1727.573 & 3774.286 & -35.804 & -71.547 & 13829.030 & -1678.620 \\
\hline 100.000 & 1695.566 & 3771.861 & 263.363 & 28.534 & 13818.830 & -1631.156 \\
\hline 110.000 & 1611.942 & 3753.943 & 554.646 & 147.144 & 1377.180 & -1531.732 \\
\hline 120.000 & 1479.219 & 3724.870 & 829.066 & 259.385 & 13712.800 & -1384.203 \\
\hline 130.000 & 1301.426 & 3688.421 & 1078.431 & 399.355 & 13633.840 & -1194.168 \\
\hline 140.000 & 1084.101 & 3652.677 & 1294.824 & 498.634 & 13553.250 & -968.792 \\
\hline 150.000 & 833.849 & 3621.557 & 1471.772 & 580.412 & 13480.460 & -715.667 \\
\hline 160.000 & 558.280 & 3599.406 & 1603.791 & 623.337 & 13423.870 & $-442 . \pi 7$ \\
\hline 170.000 & 239.398 & 3566.504 & 1683.807 & 604.637 & 12687.240 & -102.764 \\
\hline 180.000 & -83.224 & 3521.565 & 1711.795 & 562.079 & 11988.680 & 228.418 \\
\hline 190.000 & -353.294 & 3511.730 & 1693.655 & 568.739 & 12619.370 & 449.934 \\
\hline 200.000 & -615.885 & 3517.800 & 1625.991 & 675.134 & 13332.490 & 667.649 \\
\hline 210.000 & -887.776 & 3543.516 & 1505.106 & 473.615 & 13393.730 & 923.316 \\
\hline 220.000 & -1132.749 & 3578.086 & 1339.407 & 522.801 & 13472.770 & 1152.804 \\
\hline 230.000 & -1342.854 & 3627.853 & 1132.409 & 470.260 & 13569.430 & 1348.364 \\
\hline 240.000 & -1511.618 & 3679.315 & 890.146 & 278.797 & 13666.320 & 1503.510 \\
\hline 250.000 & -1634.316 & 3727.356 & 621.588 & 247.934 & 13748.860 & 1613.187 \\
\hline 260.000 & -1706.979 & 3759.993 & 333.723 & 151.865 & 13804.550 & 1672.482 \\
\hline 270.000 & -1727.573 & 3774.286 & 35.804 & 71.547 & 13829.030 & 1678.620 \\
\hline 280.000 & -1695.566 & 3771.861 & -263.363 & -28.534 & 13818.830 & 1631.156 \\
\hline 290.000 & -1611.942 & 3753.943 & -554.646 & -147.144 & 1377.180 & 1531.732 \\
\hline 300.000 & -1479.219 & 3724.870 & -829.066 & -259.385 & 13712.800 & 1384.203 \\
\hline 310.000 & -1301.426 & 3688.421 & -1078.431 & -399.355 & 13633.840 & 1194.168 \\
\hline 320.000 & -1084.101 & 3652.677 & -1294.824 & -498.634 & 13553.250 & 968.792 \\
\hline 330.000 & -833.849 & 3621.557 & -1471.772 & -580.412 & 13480.460 & 715.667 \\
\hline 340.000 & -558.280 & 3599.406 & -1603.791 & -623.337 & 13423.870 & 442.777 \\
\hline 350.000 & -239.398 & 3566.504 & -1683.807 & -604.637 & 12687.240 & 102.764 \\
\hline
\end{tabular}


Hub loads in fixed frame hub coordinates

\begin{tabular}{|c|c|c|c|c|c|c|}
\hline Azimuth Angle & Fxh & Fyh & Fzh & - Mxh & Myh & Mzh \\
\hline 0.000 & 83.224 & 3521.565 & -1711.795 & -562.079 & 11988.680 & -228.418 \\
\hline 10.000 & 53.827 & 3511.730 & -1729.274 & -638.229 & 12619.370 & -344.338 \\
\hline 20.000 & 22.621 & 3517.800 & -1738.577 & -862.768 & 13332.490 & $-396.4 \pi 5$ \\
\hline 30.000 & 16.283 & 3543.516 & -1747.348 & -871.820 & 13393.730 & -562.808 \\
\hline 40.000 & 6.782 & 3578.086 & -1754.162 & -1141.497 & 13472.770 & -547.050 \\
\hline 50.000 & -4.306 & 3627.853 & -1756.584 & -1335.184 & 13569.430 & -506.472 \\
\hline 60.000 & -15.080 & 3679.315 & -1754.173 & -1441.476 & 13666.320 & -510.310 \\
\hline 70.000 & -25.133 & 3727.356 & -1748.350 & -1600.698 & 13748.860 & -318.760 \\
\hline 80.000 & -32.239 & 3759.993 & -1738.996 & -1673.445 & 13804.550 & -140.866 \\
\hline 90.000 & -35.804 & 3774.286 & -1727.573 & -1678.620 & 13829.030 & 71.547 \\
\hline 100.000 & -35.070 & 3771.861 & -1715.539 & -1611.330 & 13818.830 & 255.147 \\
\hline 110.000 & -30.120 & 3753.943 & -1704.430 & -1489.683 & 13777.180 & 385.613 \\
\hline 120.000 & -21.617 & 3724.870 & -1695.574 & -1328.447 & 13712.800 & 467.467 \\
\hline 130.000 & -10.415 & 3688.421 & -1690.152 & -1171.486 & 13633.840 & 461.672 \\
\hline 140.000 & 1.828 & 3652.677 & -1688.740 & -1004.703 & 13553.250 & 421.622 \\
\hline 150.000 & 13.752 & 3621.557 & -1691.517 & -860.485 & 13480.460 & 329.580 \\
\hline 160.000 & 23.918 & 3599.406 & -1698.013 & -737.183 & 13423.870 & 202.880 \\
\hline 170.000 & 56.629 & 3566.504 & -1699.797 & -613.296 & 12687.240 & -3.792 \\
\hline 180.000 & 83.224 & 3521.565 & -1711.795 & -562.079 & 11988.680 & -228.417 \\
\hline 190.000 & 53.827 & 3511.730 & -1729.274 & -638.229 & 12619.370 & -344.338 \\
\hline 200.000 & 22.621 & 3517.800 & -1738.577 & -862.768 & 13332.490 & -396.475 \\
\hline 210.000 & 16.283 & & -1747.348 & -871.820 & & -562.808 \\
\hline 220.000 & 6.782 & 3578.086 & -1754.162 & -1141.497 & 13472.770 & -547.050 \\
\hline 230.000 & -4.306 & 3627.853 & -1756.584 & -1335.184 & 13569.430 & -506.471 \\
\hline 240.000 & -15.080 & 3679.315 & -1754.173 & -1441.476 & 13666.320 & -510.310 \\
\hline 250.000 & -25.133 & 3727.356 & -1748.350 & -1600.698 & 13748.860 & -318.760 \\
\hline 260.000 & -32.239 & 3759.993 & -1738.996 & -1673.445 & 13804.550 & -140.866 \\
\hline 270.000 & -35.804 & 3774.286 & -1727.573 & -1678.620 & 13829.030 & 71.547 \\
\hline 280.000 & -35.070 & 3771.861 & -1715.539 & -1611.330 & 13818.830 & 255.147 \\
\hline 290.000 & -30.120 & 3753.943 & -1704.430 & -1489.683 & 1377.180 & 385.613 \\
\hline 300.000 & -21.617 & 3724.870 & -1695.574 & -1328.447 & 13712.800 & 467.467 \\
\hline 310.000 & -10.414 & 3688.421 & -1690.152 & -1171.487 & 13633.840 & 461.672 \\
\hline 320.000 & 1.828 & 3652.677 & -1688.740 & -1004.703 & 13553.250 & 421.622 \\
\hline 330.000 & 13.753 & 3621.557 & -1691.517 & -860.485 & 13480.460 & 329.579 \\
\hline 340.000 & 23.918 & 3599.406 & -1698.014 & -737.183 & 13423.870 & 202.880 \\
\hline
\end{tabular}




$\begin{array}{lllllll}350.000 & 56.630 & 3566.504 & -1699.797 & -613.296 & 12687.240 & -3.792 \\ 360.000 & 83.224 & 3521.565 & -1711.795 & -562.079 & 11988.680 & -228.417\end{array}$

Harmonics of Rotor Shaft Loads

\begin{tabular}{lrrrrrr} 
& \multicolumn{1}{l}{ Fxr } & \multicolumn{1}{c}{ Fyr } & \multicolumn{1}{l}{ Fzr } & \multicolumn{1}{l}{ Mxr } & \multicolumn{1}{c}{ Myr } & \multicolumn{1}{c}{ Mzr } \\
$\cos (0 *$ PSI) & 0.000 & 3645.596 & 0.000 & 0.000 & 13417.380 & 0.000 \\
$\sin (0 . * P S I)$ & 0.000 & 0.000 & 0.000 & 0.000 & 0.000 & 0.000 \\
$\cos (P S I)$ & 42.884 & 0.000 & -1717.292 & -625.288 & -0.001 & -144.024 \\
$\sin (P S I)$ & 1726.108 & 0.000 & -35.208 & -36.940 & 0.000 & -1666.094 \\
$\cos (2 . * P S I)$ & 0.000 & -121.367 & 0.000 & 0.000 & -543.361 & 0.000 \\
$\sin (2 . * P S I)$ & 0.000 & -36.943 & 0.000 & 0.000 & -40.345 & 0.000 \\
$\cos (3 . * P S I)$ & 10.331 & 0.000 & 1.834 & 0.763 & 0.000 & -22.828 \\
$\sin (3 . * P S I)$ & -2.286 & 0.000 & 0.792 & 28.029 & 0.001 & 14.056 \\
$\cos (4 . * P S I)$ & 0.000 & 7.607 & 0.000 & 0.000 & -255.006 & 0.000 \\
$\sin (4 . * P S I)$ & 0.000 & -12.744 & 0.000 & 0.000 & -15.211 & 0.000 \\
$\cos (5 . * P S I)$ & 9.647 & 0.000 & 1.363 & 31.431 & 0.000 & -19.841 \\
$\sin (5 . * P S I)$ & -1.224 & 0.000 & 0.376 & -1.303 & 0.000 & 2.575
\end{tabular}

Harmonics of Hub loads in Fixed Coordinates

\begin{tabular}{lrrrrrr} 
& \multicolumn{1}{l}{ Fxh } & \multicolumn{1}{l}{ Fyh } & \multicolumn{1}{l}{ Fzh } & \multicolumn{1}{l}{ Mxh } & \multicolumn{1}{l}{ Myh } & \multicolumn{1}{l}{ Mzh } \\
cos(0*PSI) & 3.838 & 3645.596 & -1721.700 & -1145.691 & 13417.380 & -53.542 \\
$\sin (0 . * P S I)$ & 0.000 & 0.000 & 0.000 & 0.000 & 0.000 & 0.000 \\
$\cos (P S I)$ & 0.000 & 0.000 & 0.000 & 0.000 & 0.000 & 0.000 \\
$\sin (P S I)$ & 0.000 & 0.000 & 0.000 & 0.000 & 0.000 & 0.000 \\
$\cos (2 . * P S I)$ & 44.607 & -121.367 & 6.469 & 527.812 & -543.363 & -115.911 \\
$\sin (2 . * P S I)$ & 2.348 & -36.943 & -33.484 & -65.054 & -40.345 & -512.993 \\
$\cos (3 . * P S I)$ & 0.000 & 0.000 & 0.000 & 0.000 & -0.001 & 0.000 \\
$\sin (3 . * P S I)$ & 0.000 & 0.000 & 0.000 & 0.000 & 0.001 & 0.000 \\
$\cos (4 . * P S I)$ & 9.781 & 7.606 & 1.067 & 10.356 & -255.007 & -6.669 \\
$\sin (4 . * P S I)$ & -1.519 & -12.744 & 0.242 & 11.869 & -15.211 & 23.649 \\
$\cos (5 . * P S I)$ & 0.000 & 0.000 & 0.000 & 0.000 & -0.001 & 0.000 \\
$\sin (5 . * P S I)$ & 0.000 & 0.000 & 0.000 & 0.000 & 0.000 & 0.000
\end{tabular}


THIS IS THE TURBULENT LOADS OUTPUT FILE

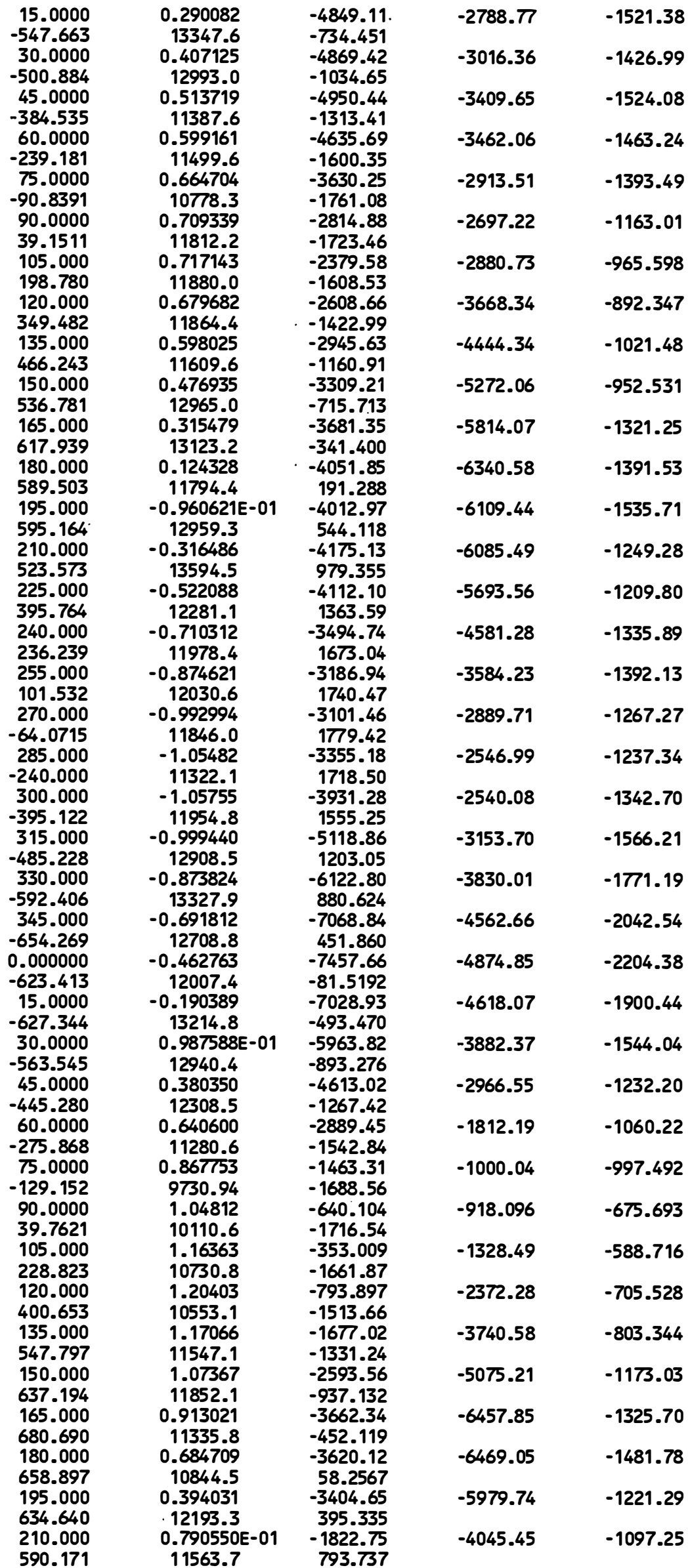




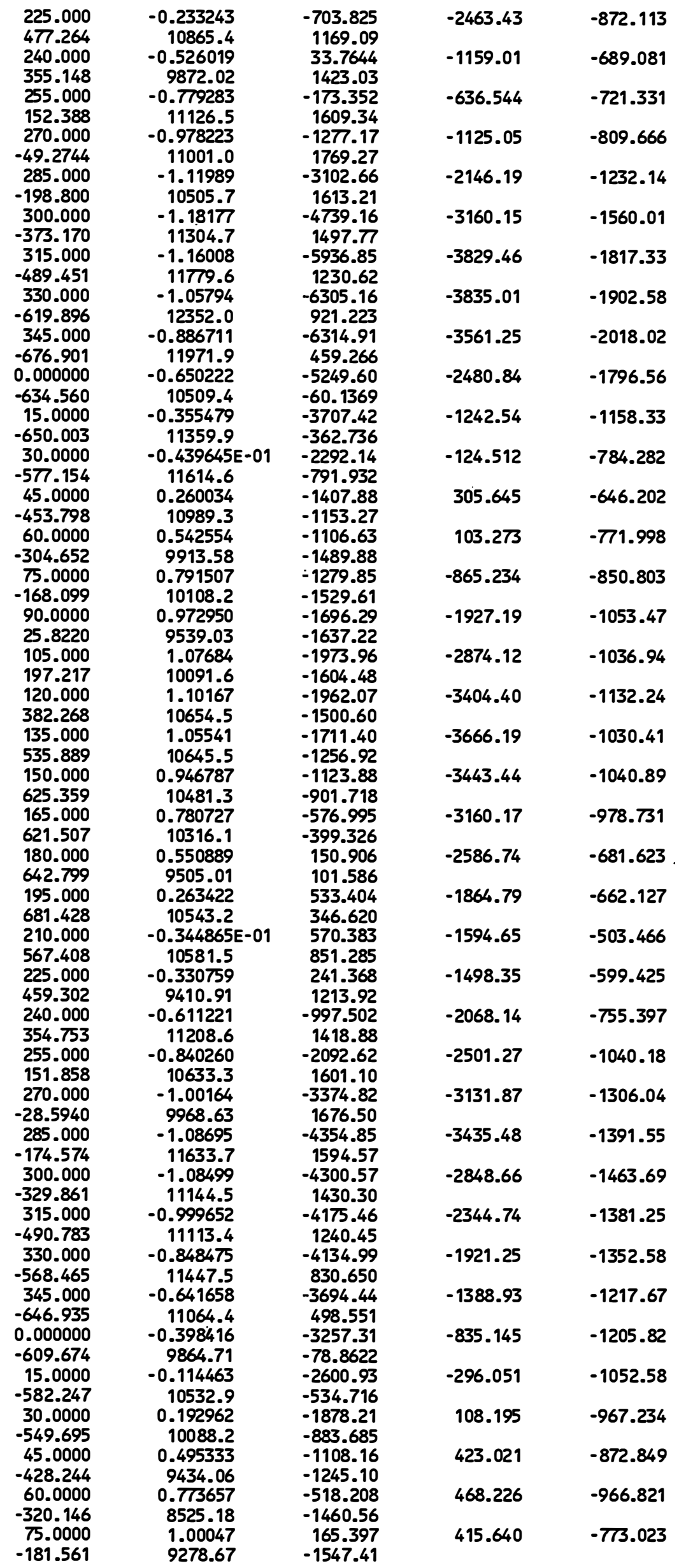




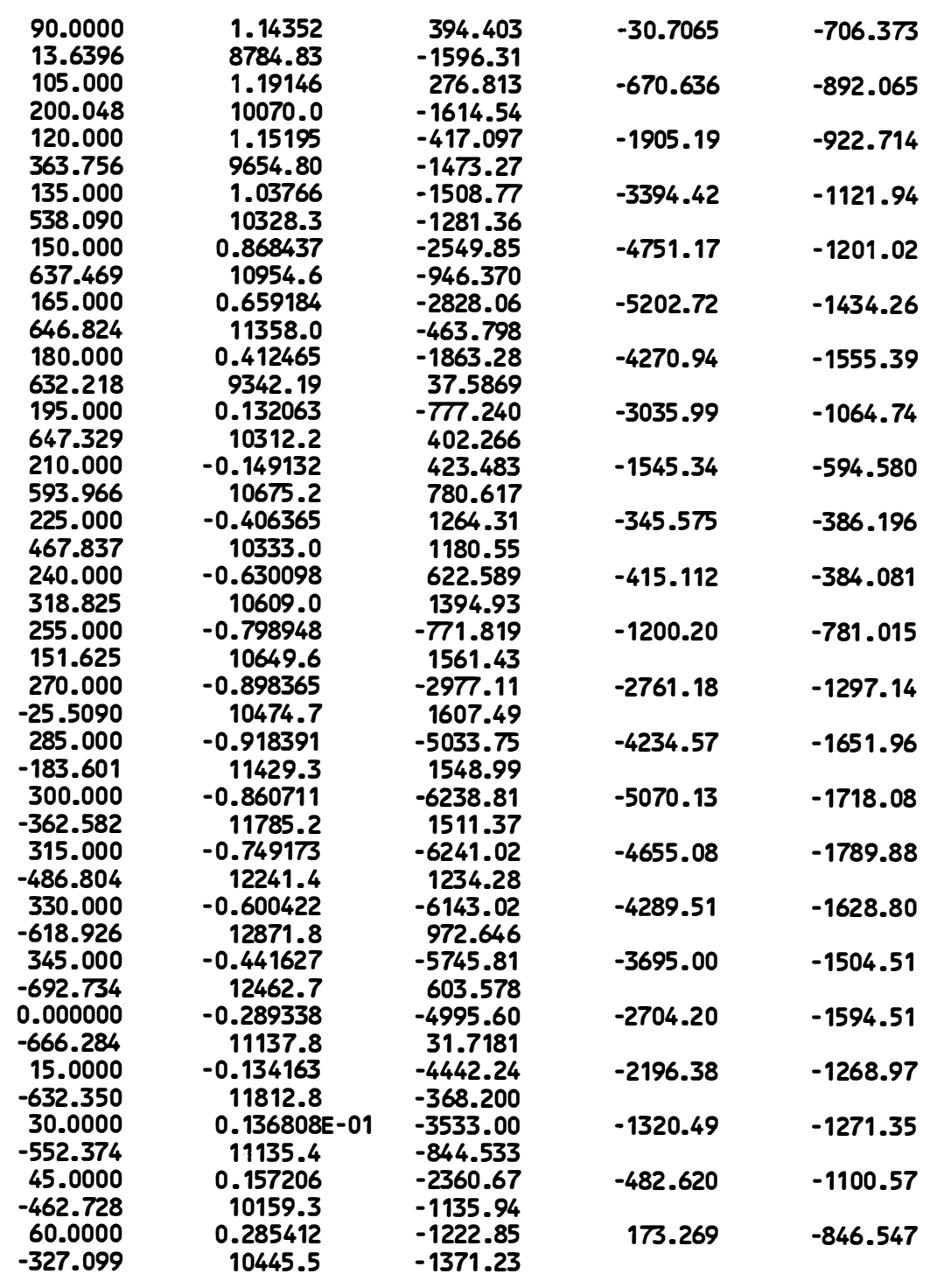




\section{Appendix C}

Module 1 Listing

C-1 
Module 1

This program is the first of two modules that constitute the STRAP code. This first module interpolates the * input data into a form usable by the second module * which does the actual modeling. This module also com- * putes the coefficient matrix used for solving the blade flaps equation of motion.

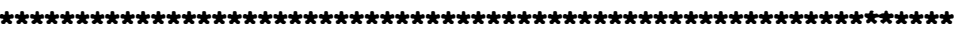

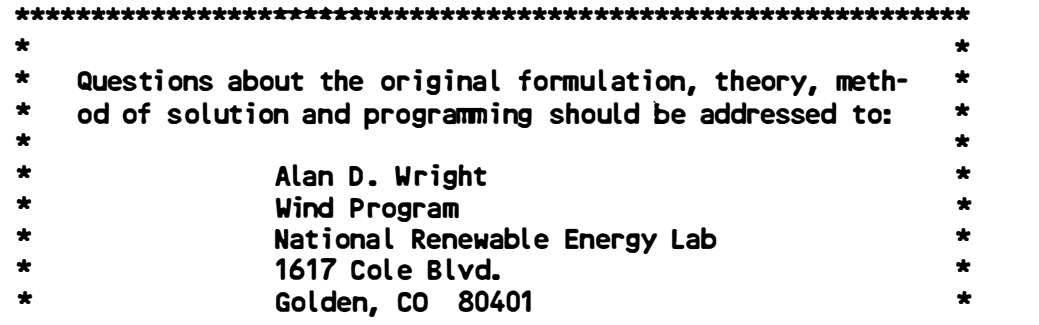




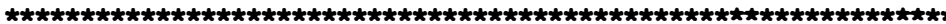

\section{I/O Conventions:}

Unit 1 - Input data file

Unit 2 - Output data file

Unit 5 - Keyboard

Unit * - Monitor or keyboard

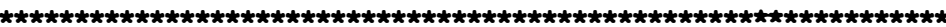

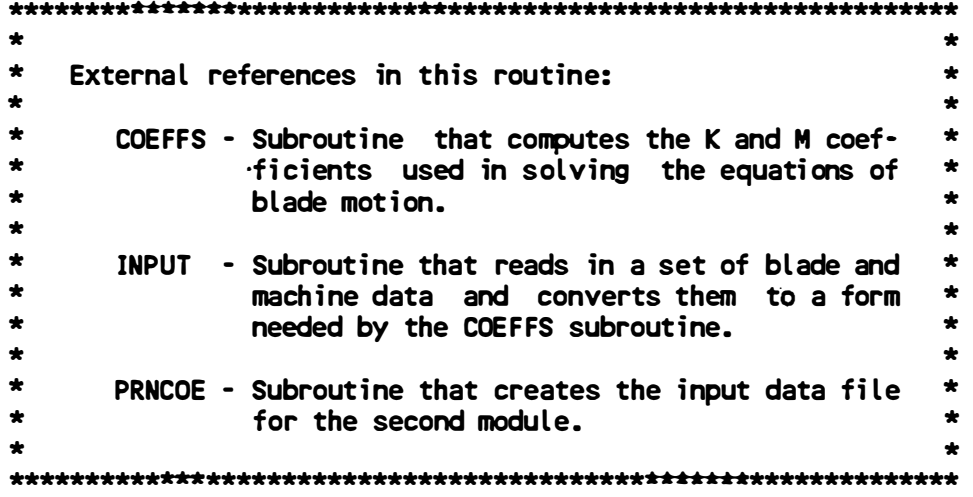

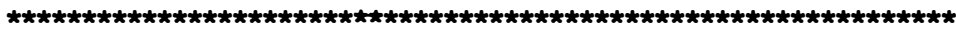

$\star$

Local and dumy variables used in this routine:

ANS - Used to store input responses from the keyboard.

FILOUT - String that contains the name of the output file. It is defined in INPUT.

NP - Number of blade property values used in performing composite Simpson's integration for the $K$ and $M$ coefficient arrays. This number is approximately 10 times the NPTS value.

NPTS - Number of points along the blade used to perform Simpson's integration for calculating the moments and forces at the blade root

NSIMP - Order of the composite simpson's integration used in the run. Parameter is set to 10 in order to make 21 blade property staions.

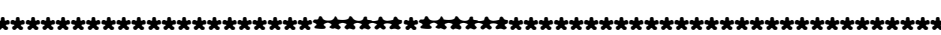

$\begin{array}{ll}\text { INTEGER } & \text { NP } \\ \text { INTEGER } & \text { NPTS } \\ \text { INTEGER } & \text { NSIMP }\end{array}$

CHARACTER*1 ANS

CHARACTER $* 50$ FILOUT

PARAMETER ( NSIMP $=10)$

100 FORMAT

\& If Program For Analysis of a 2-Bladed Teetering Hub Rotor' \& $/$ Dynamic Response and Loads Analysis'

\& $/ /$ ' MODULE 1- STRAP1')

110 FORMAT $(/)$

120 FORMAT ( A )

130 FORMAT ( / ' STRAP1 terminated normally.' / )

C Calculate the number of blade property stations and interpola-

C tion points. 
NPTS $=2 *$ NSIMP +1

$N P=20 * N S I M P+1$

Print title.

PRINT 100

Get input responses, generate coefficients, and write to output filë.

10 PRINT 110

CALL INPUT ( NP , NPTS , FILOUT )

CALL MODES (NP)

CALL COEFFS ( NP, NPTS )

CALL PRNCOE ( FILÓUT , NPTS )

Check to see if user wants to process another data file.

20 PRINT *, , ,

PRINT *, 'Do you want to process another data file? $(\mathrm{Y},=\mathrm{N})>$ '

READ 120, ANS

IF ( ( ANS .EQ. 'Y' ) .OR. ( ANS .EQ. ' $Y$ ') ) GO TO 10

\&

IF ( ( ANS .NE. 'N') .AND. ( ANS .NE. ' $n$ ')

.AND. ( ANS .NE. ' , ) ) THEN

PRINT *, 'Invalid response. Please try again...'

END IF GO TO 20

C

Processing complete.

PRINT 130

STOP

END

BLOCK DATA

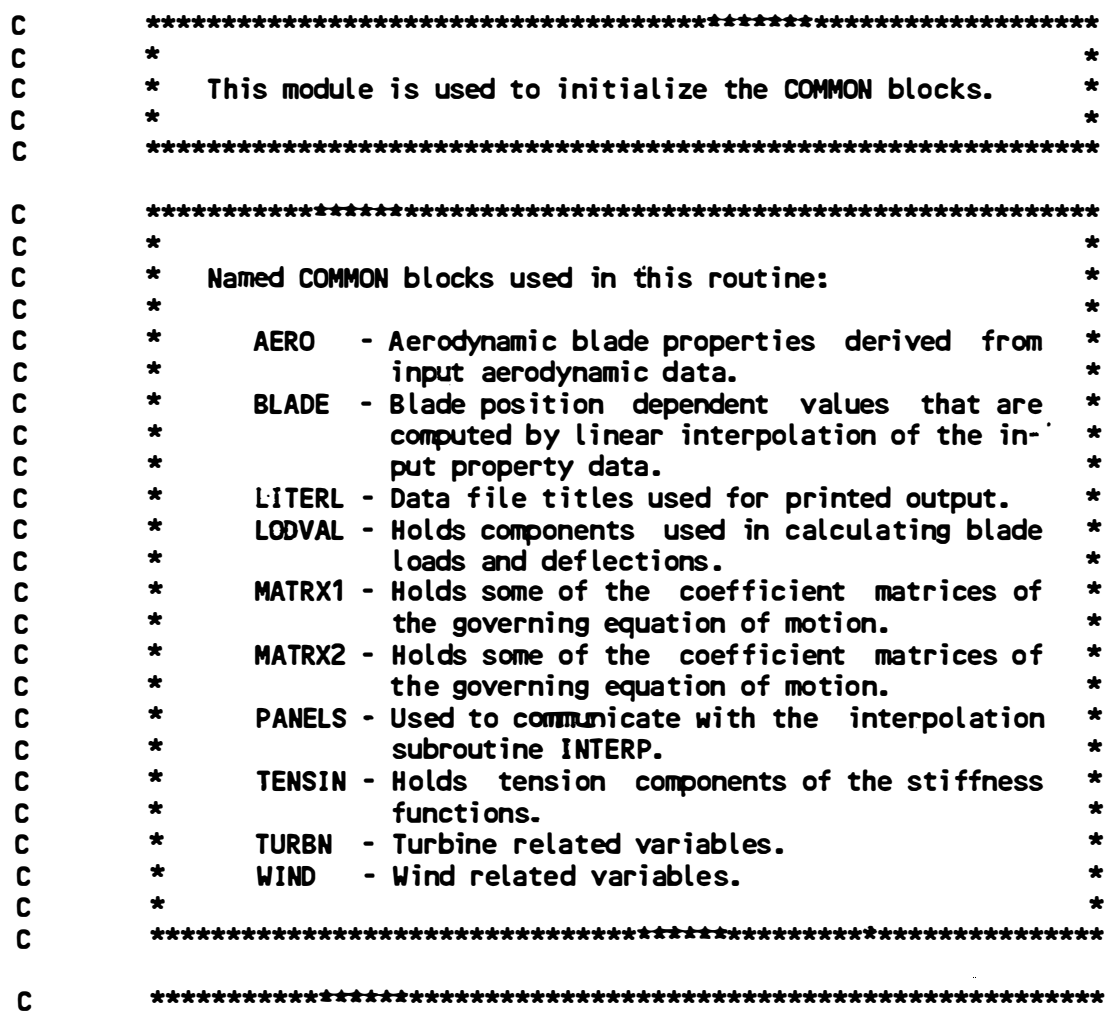




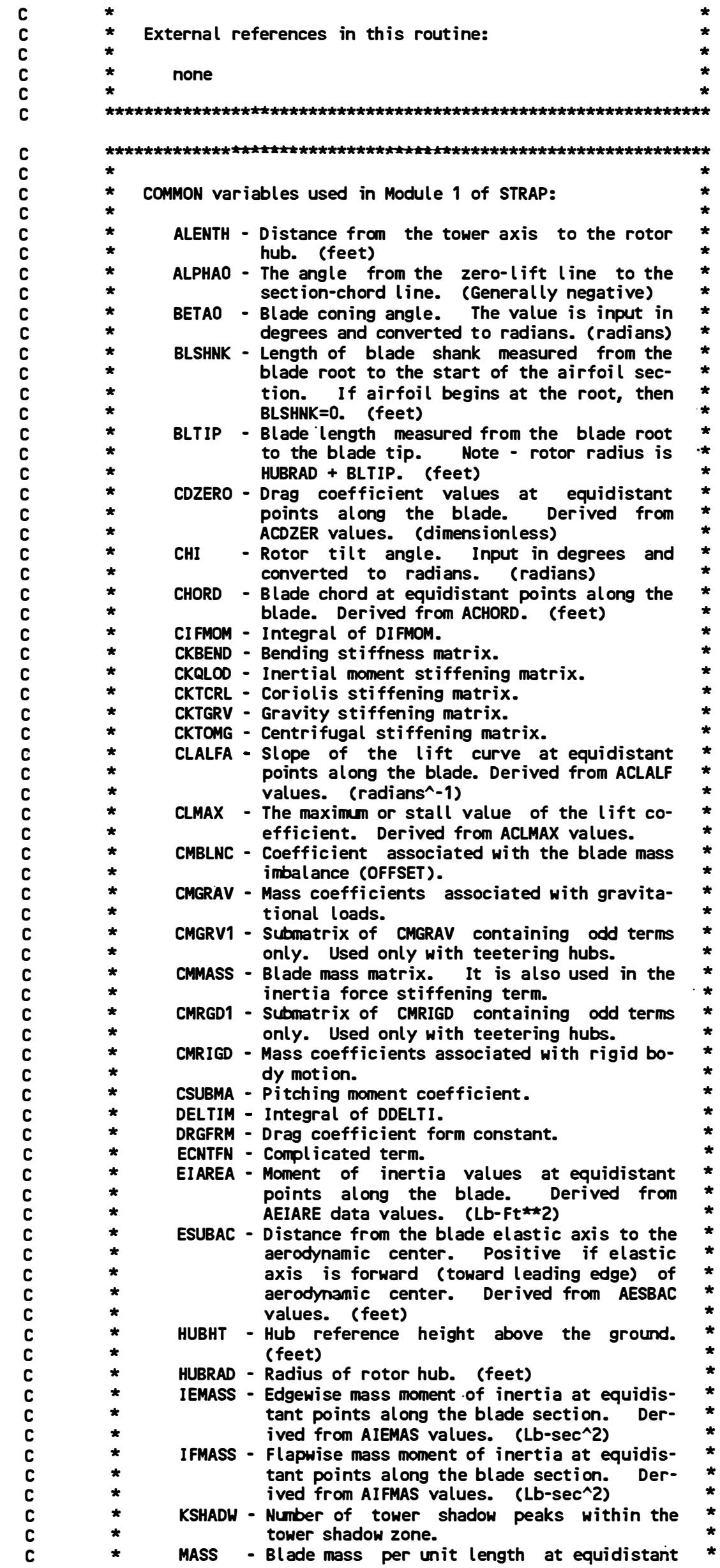


points along the blade. Input in Lbf/ft, via the input variable WEIGHT, and converted to slugs/ft. (slugs/ft)

NBLADS - Number of turbine blades.

NSHAPS - Number of blade shape functions, 4 maximum.

OFFMAS - Integral of DOFFMS.

OFFSET - Distance from the elastic axis to the mass axes of blade section. Positive towards the leading edge. Derived from AOFFST values. Called E-sub-eta in the formulation. (feet)

OMEGA - Rotor speed. Input in RPM and converted to radians/second. (rad/sec)

PHIO - Rotor mean yaw angle. (radians)

PHIAMP - Amplitude of periodic yaw motion about mean yaw angle. Input as degrees and converted to radians. (radians)

PHIOMg - Maximm yaw rate. Used internally to compute the yaw period. It can be used to input a turbine steady yaw rate. Input as degrees/sec and converted to radians/second. (radians/sec)

PSIZER - Half angle width of the tower shadow region. (degrees)

SHERXP - Wind shear power exponent.

STEP - Distance between the equidistant data points along the blade. (feet)

TCORLS - Blade tension coefficient due to coriolis effects.

TGRAV - Blade tension coefficient due to gravity effects.

THETAO - Orientation of the zero lift line with respect to the blade principal bending axis.

THETAP - The angle

from the bending axis, XP, to the cone of rotation, $X$-axis, for the reference station. THETAP establishes the orientation of the flapping displacements. Positive angles are toward feather. Generally, the reference station section-chord line is taken as the bending (flapping) axis. (radians)

THETAT - The built-in blade twist angle from the section-chord line at the reference station to the section-chord line at the tip of the rotor. Positive towards feather.

TITLE1 - First line of the data file title.

TITLE2 - Second line of the data file title.

TITLE3 - Third line of the data file title.

TOMGA - Blade tension coefficient associated with centrifugal force effects.

TSUBO - Tower shadow wind speed offset component.

TSUBP - Tower shadow sinusoidal component.

VHUB - Air speed at the height of the hub. ( ft/sec)

XLEFT - Radial positions of the blade property data points. These are monotonically increasing values from 0.0 at the root to $R$ at the tip. (feet) 


$\begin{array}{ll}\text { INCLUDE } & \text { 'C:INCLUDEITENSIN.INC' } \\ \text { INCLUDE } & \text { 'C:INCLUDEITURBN.INC' } \\ \text { INCLUDE } & \text { 'C:INCLUDEIWIND.INC' }\end{array}$

END

SUBROUTINE CAPS ( STRING )

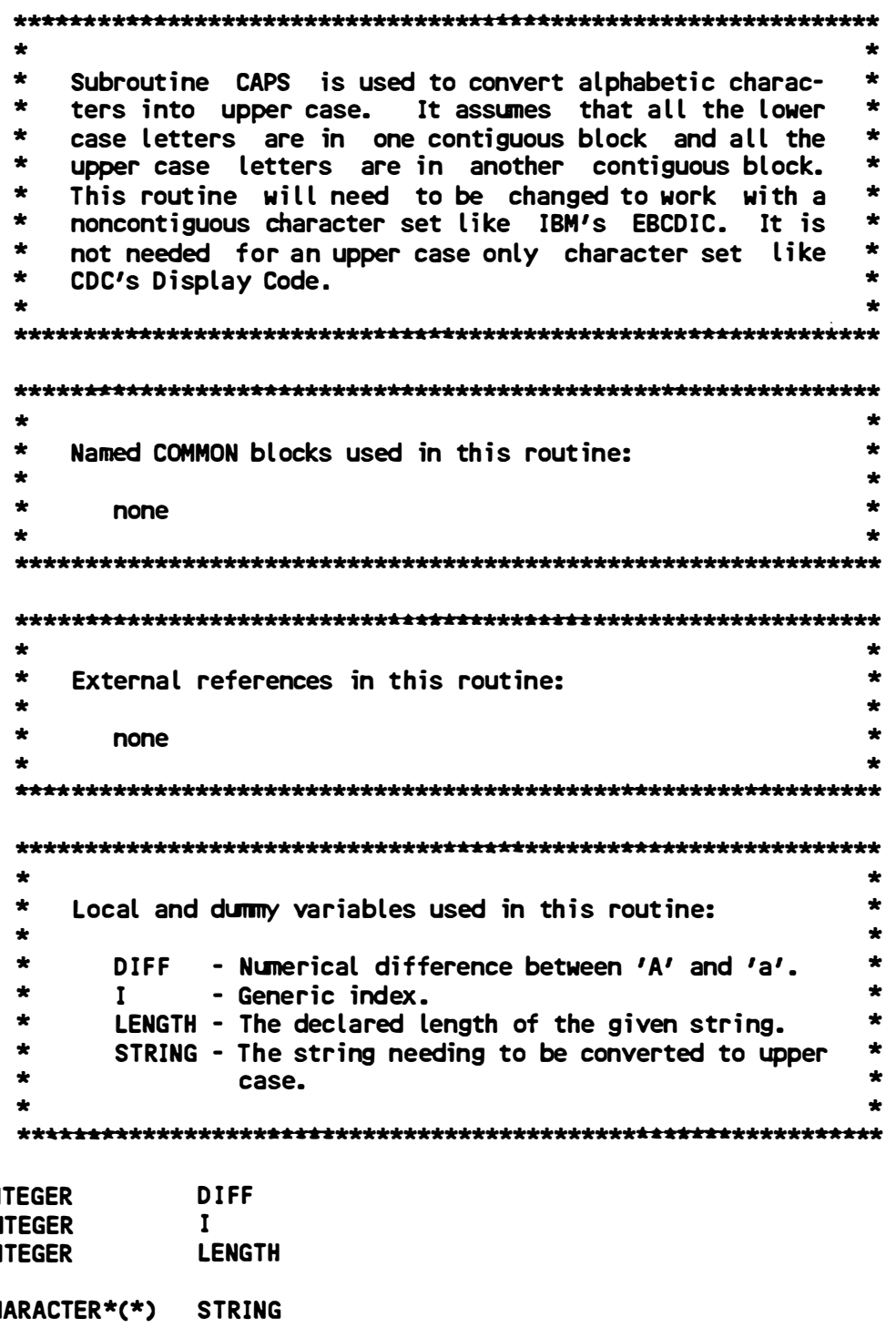

C Compute the numerical difference between 'A' and ' $a$ '.

DIFF = ICHAR( ' $a$ ' ) - ICHAR( 'A' )

C Get the length of the string.

LENGTH = LEN( STRING )

C Look for lower case letters. Convert them to upper case.

DO 100 I=1,LENGTH

\&

IF ( ( STRING(I:I) .GE. ' $a$ ' ) .AND.

( STRING(I:I) .LE. ' 2 ') ) THEN

STRING(I:I) = CHAR( ICHAR( $\operatorname{STRING(I:I))-DIFF~)~}$ 
SIMPSN - Function that performs the composite Simpson's integration on the input data arrays.

TRPZOD - Function that performs composite trapezoidal integration.

BLTBLT - Square of BLTIP. (feet**2)

DDELTI - IEMASS - IFMASS.

DIFMOM - Complicated term.

DKBEND - Used to compute the integrals associated with the coefficient matrices.

DKMASS - Used to compute the integrals associated with the coefficient matrices.

DKQLD - Used to compute the integrals associated with the coefficient matrices.

DKTCRL Used to compute the integrals associated with the coefficient matrices.

DKTGRA - Used to compute the integrals associated with the coefficient matrices.

DKTOMG - Used to compute the integrals associated with the coefficient matrices.

DMBALN - Used to compute the integrals associated with the coefficient matrices.

DMGRAV - Used to compute the integrals associated with the coefficient matrices. 


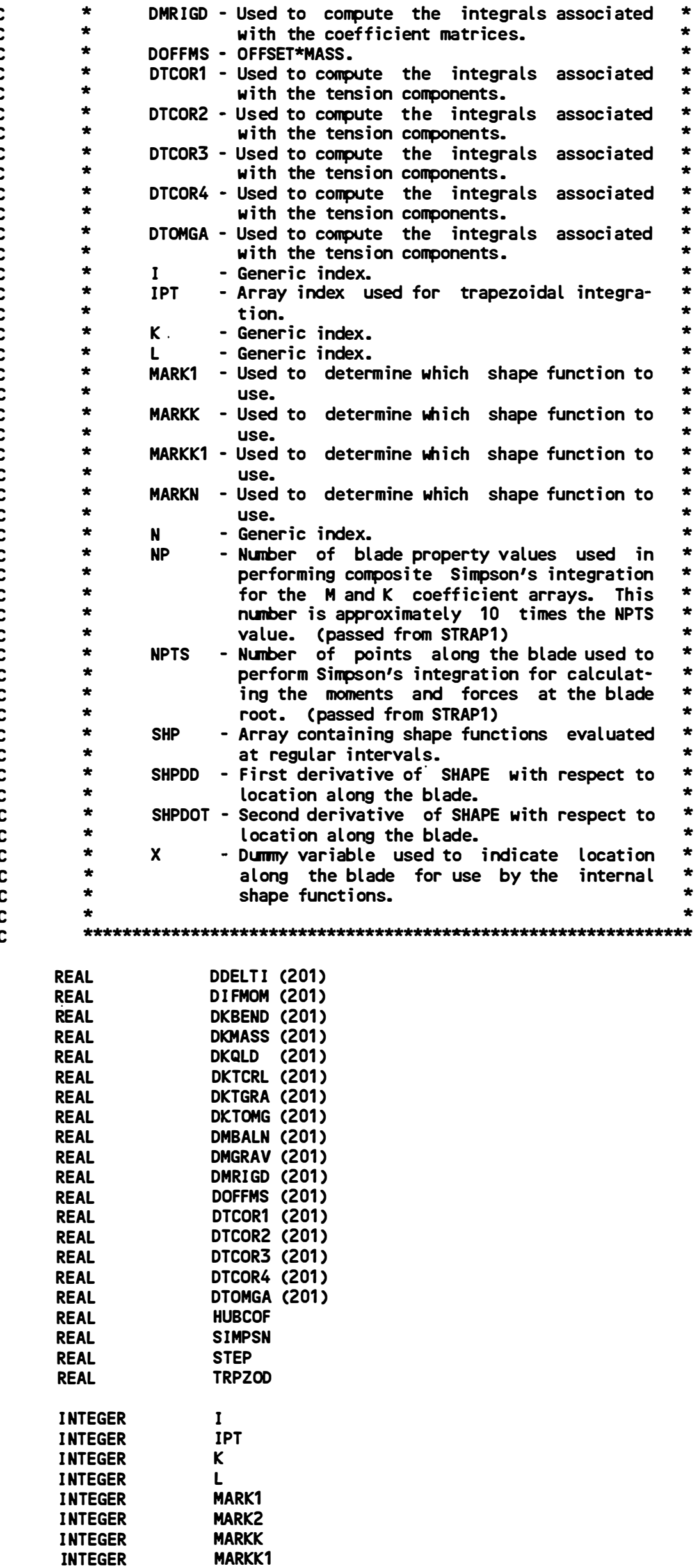




\begin{tabular}{|c|c|}
\hline $\begin{array}{l}\text { INTEGER } \\
\text { INTEGER } \\
\text { INTEGER } \\
\text { INTEGER } \\
\text { INTEGER }\end{array}$ & $\begin{array}{l}\text { MARKN } \\
\text { N } \\
\text { NFLAG } \\
\text { NP } \\
\text { NPTS }\end{array}$ \\
\hline INCLUDE & 'C:INCLUDE\AERO. INC' \\
\hline INCLUDE & 'C:INCLUDE \\
\hline INCLUDE & 'C:INCLUDE\CONST.INC' \\
\hline INCLUDE & 'C:INCLUDEILODVAL .INC' \\
\hline INCLUDE & 'C:INCLUDE MATRX1. INC' \\
\hline INCLUDE & 'C:INCLUDEXMATRX2.INC' \\
\hline INCLUDE & 'C: INCLUDE TTENSIN. INC' \\
\hline INCLUDE & 'C: INCLUDE YMODAL.INC' \\
\hline INCLUDE & 'C:INCLUDE \\
\hline INCLUDE & 'C:INCLUDE ITURBN.INC' \\
\hline INCLUDE & 'C:INCLUDEISPRING.INC' \\
\hline
\end{tabular}

1000 FORMAT ( / ' Generating coefficient matrices and property' \& 8000 FORMAT (' '\&done.')

C
C
C
C
C

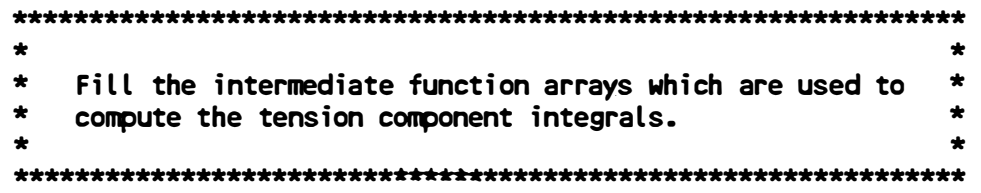

STEP $=$ BLTIP $/(N P-1.0)$

DO $200 I=1$, NP

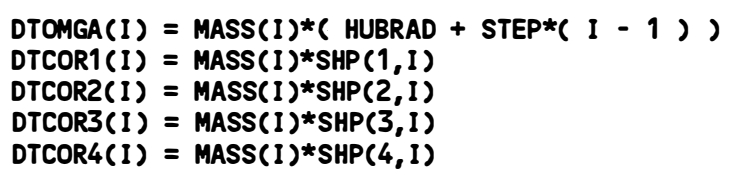

200 CONTINUE

C
C
C
C
C
C
C

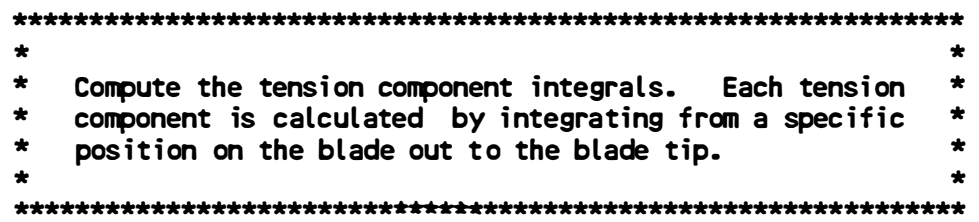

DO $300 \quad I=1, N P$

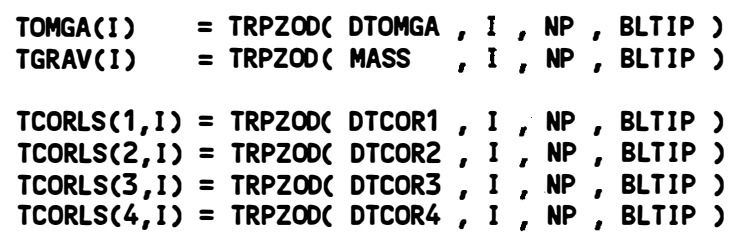

300 CONTINUE 
NFLAG $=1$

DO $470 K=1,4$

DO $440 \mathrm{~L}=K, 4$

MARK1 $=1+\operatorname{NFLAG*}(-1) * *(K+L)$

MARK2 $=1+\operatorname{NFLAG*}(-1) * *(K+L+1)$

IF ( MARK1 .NE. O ) THEN

Fill the intermediate function arrays which are used to compute the stiffness and loading coefficient matrices.

DO $400 I=1$, NP

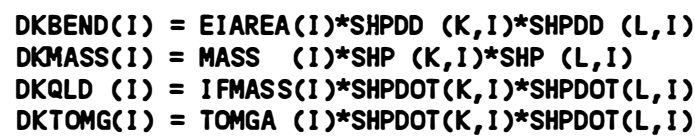

CONTINUE

C

Compute the K,Lth element of the coefficient matrices.

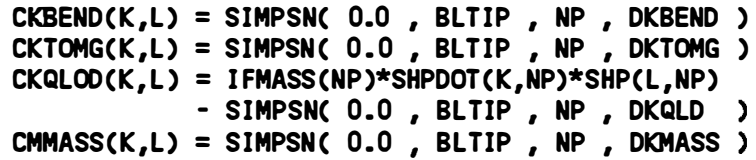

ELSE

$\operatorname{CKBEND}(K, L)=0.0$

$\operatorname{CKTOMG}(K, L)=0.0$

$\operatorname{CKQLOD}(K, L)=0.0$

$\operatorname{CMMASS}(K, L)=0.0$

END IF

IF ( MARK2 . NE. O ) THEN

$$
\begin{aligned}
& \text { DO } 410 I=1, N P \\
& \operatorname{DKTGRA}(I)=\operatorname{TGRAV}(I) * \operatorname{SHPDOT}(K, I) * \operatorname{SHPDOT}(L, I)
\end{aligned}
$$

$\operatorname{CKTGRV}(K, L)=\operatorname{SIMPSN}(0.0, \operatorname{BLTIP}, \mathrm{NP}, \mathrm{DKTGRA})$

ELSE

$\operatorname{CKTGRV}(K, L)=0.0$

END IF

C

Index into coefficient matrix for coriolis stiffening.

DO $430 \mathrm{~N}=1,4$

MARKN $=1+\operatorname{NFLAG*}(-1) * *(K+L+N)$

IF ( MARKN .NE. O ) THEN

$\operatorname{CKTCRL}(N, K, L)=\operatorname{SIMPSN}(0.0$, BLTIP , NP , DKTCRL )

ELSE 
$\operatorname{CKTCRL}(N, K, L)=0.0$

ENDIF

430

CONTINUE

$\operatorname{CKTCRL}(1, K, L)=0$.

440

CONTINUE

C Compute elements of coefficient matrices which use only a

C

C single index. Fill intermediate function arrays first, then compute the elements of the matrices.

MARKK $=1+\operatorname{NFLAG*}(-1) * * K$

MARKK1 $=1+\operatorname{NFLAG*}(-1) * *(K+1)$

IF ( MARKK .NE. 0 ) THEN

DO $450 I=1, N P$

$\operatorname{DMRIGD}(I)=\operatorname{MASS}(I) *($ HUBRAD $+\operatorname{STEP} *(I-1)) * \operatorname{SHP}(K, I)$

$\operatorname{DMBALN}(I)=\operatorname{MASS}(I) * \operatorname{SHP}(K, I) *$ OFFSET $(I)$

$\operatorname{DMGRAV}(I)=\operatorname{MASS}(I) * \operatorname{SHP}(K, I)$

450

CONTINUE

$\operatorname{CMRIGD}(K)=\operatorname{SIMPSN}(0.0$, BLTIP, NP, DMRIGD )

CMBLNC(K) $=$ SIMPSN( 0.0, BLTIP, NP , DMBALN )

CMGRAV(K) $=$ SIMPSNC 0.0, BLTIP, NP， DMGRAV;

ELSE

$\operatorname{CMRIGD}(K)=0$.

$\operatorname{CMBLNC}(K)=0$.

$\operatorname{CMGRAV}(K)=0$.

ENDIF

IF ( MARKK1 .NE. O ) THEN

DO $460 \cdot I=1$,NP

$\operatorname{DMRIGD}(I)=\operatorname{MASS}(I) *($ HUBRAD $+\operatorname{STEP} *(I-1)) * \operatorname{SHP}(K, I)$

$\operatorname{DMBALN}(I)=\operatorname{MASS}(I) * \operatorname{SHP}(K, I) * \operatorname{OFFSET}(I)$

$\operatorname{DMGRAV}(I)=\operatorname{MASS}(I) * S H P(K, I)$

460

CONTINUE

CMRGD1(K) $=$ SIMPSN( 0.0, BLTIP , NP , DMRIGD )

CMGRVI $(K)=$ SIMPSN( 0.0, BLTIP，NP，DMGRAV )

ELSE

$\operatorname{CMRGD1}(K)=0.0$

$\operatorname{CMGRV1}(K)=0.0$

.ENDIF

470 CONTINUE

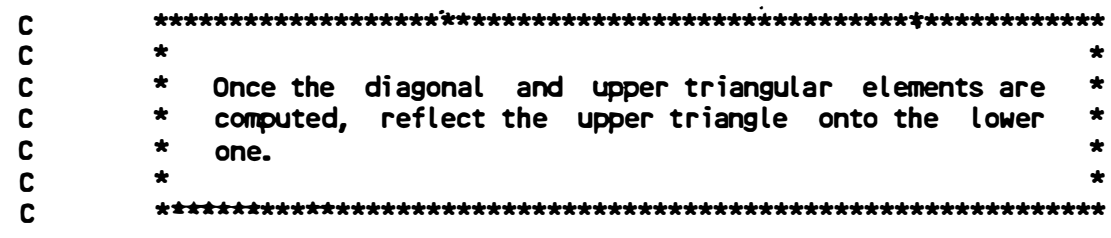

DO $520 K=1,3$

DO $510 L=K+1,4$

$\operatorname{CKBEND}(L, K)=\operatorname{CKBEND}(K, L)$ 

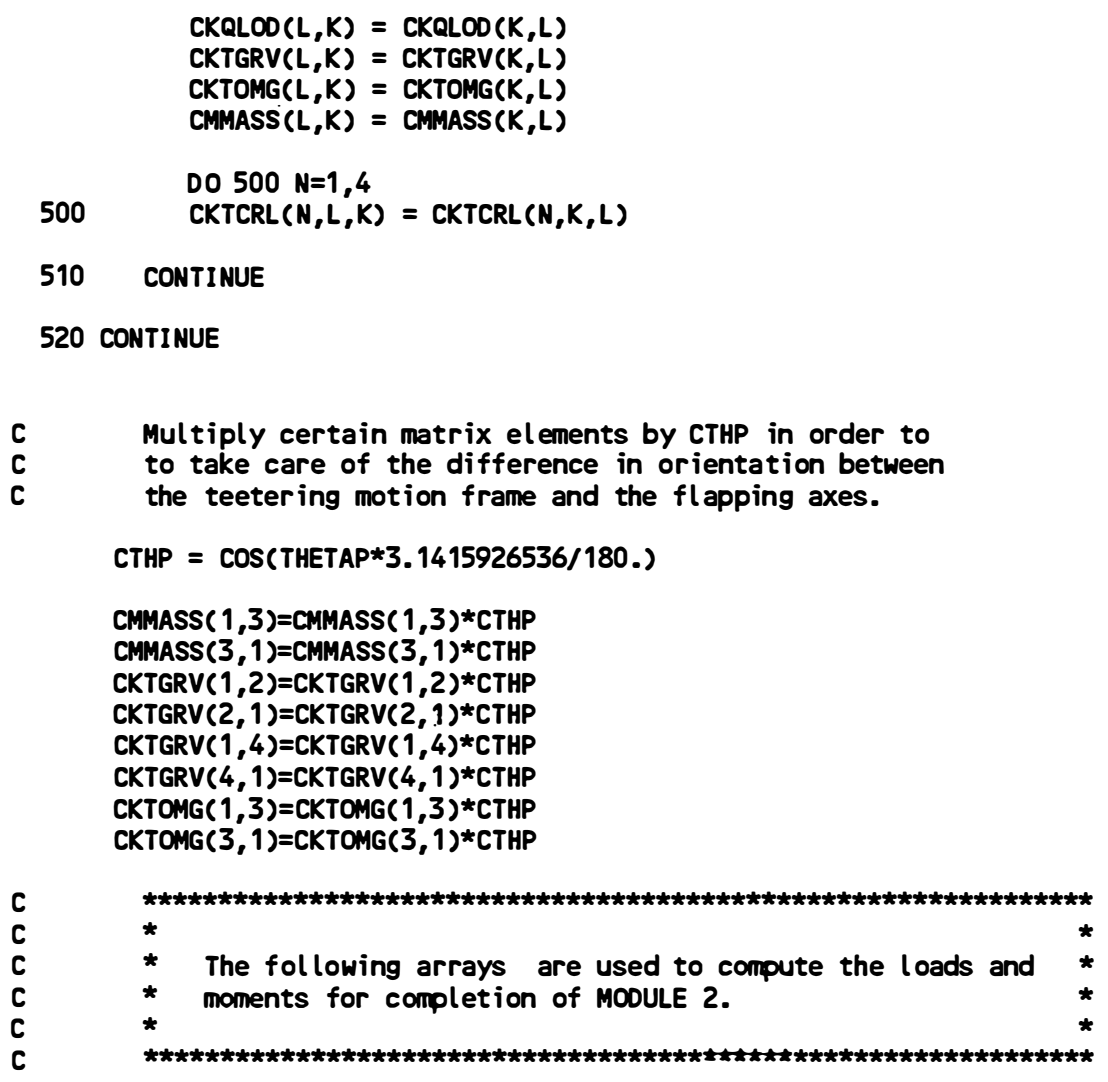

DO $600 I=1, N P$

$\operatorname{DDELTI}(I)=\operatorname{IEMASS}(I)-I$ MMASS $(I)$

$\operatorname{DOFFMS}(I)=\operatorname{OFFSET}(I) * \operatorname{MASS}(I)$

600 CONTINUE

DO 610 I=1,NPTS

IPT $=10 *(1-1)+1$

DELTIM(I) $=\operatorname{TRPZOD(~DDELTI,~IPT~,~NP~,~BLTIP~)~}$

OFFMAS(I) $=\operatorname{TRPZOD}($ DOFFMS , IPT; NP , BLTIP)

610 CONTINUE

DO $640 \mathrm{~N}=1,4$

DO $620 \mathrm{I}=1$, NP

620 DIFMOM(I) = IFMASS (I)*SHPDOT $(N, I)$

DO $630 I=1$,NPTS

IPT $=10 *(I-1)+1$

$\operatorname{CIFMOM}(N, I)=\operatorname{TRPZOO}($ DIFMOM , IPT , NP , BLTIP )

630 CONTINUE

640 CONTINUE

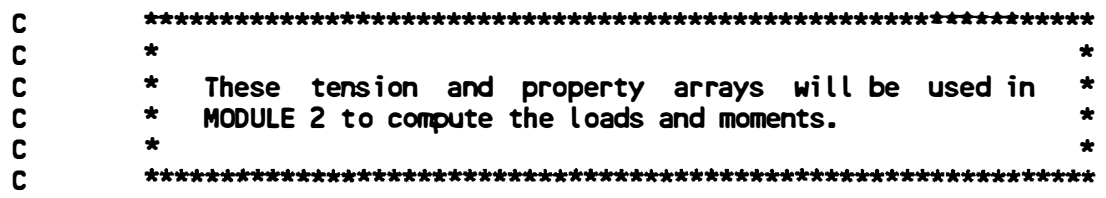

DO $700 \mathrm{I}=1$, NPTS

IPT $=10 *(I-1)+1$ 


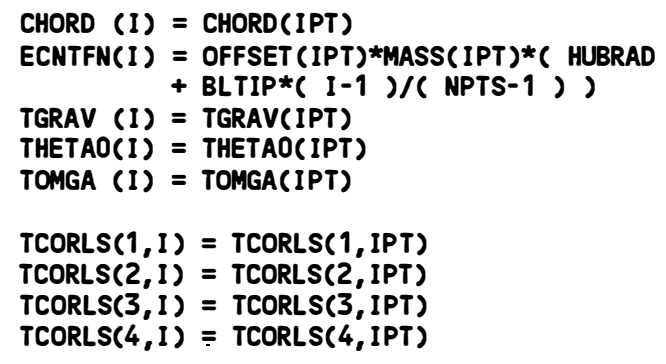

700 CONTINUE

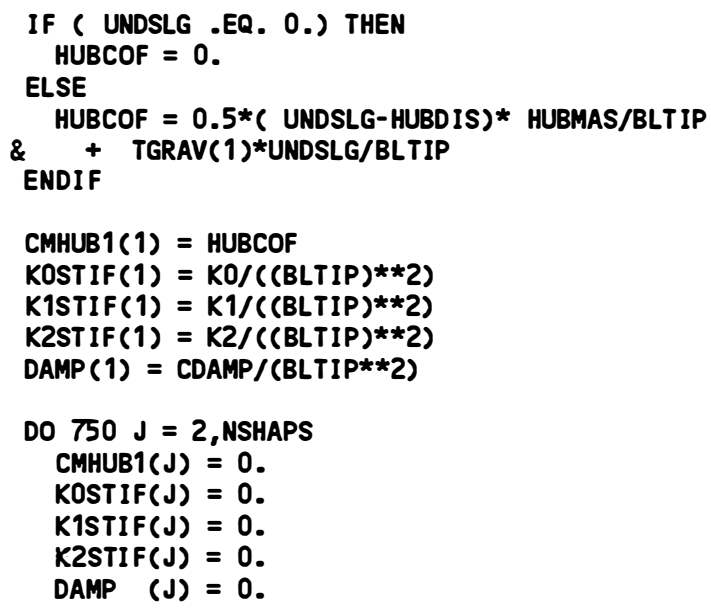

SUBROUTINE MODES(NP)

REAL CF1

REAL CF2

REAL CF2DOT

REAL CF2DD

REAL CF3

REAL CF3DOT

REAL CF3DD

REAL CF4

REAL CF4DOT

REAL CF4DD

REAL CF5

REAL CF5DOT

REAL CF5DD

REAL ASUM( 8,8 )

REAL BSUM $(8,8)$

REAL CSUM $(8,8)$

REAL DSUM $(8,8)$

REAL BLTBLT

REAL EKBEND(201)

REAL EKMASS (201)

REAL EKTOMG(201)

REAL ETOMGA(201)

REAL MAG(8)

REAL OMGA2

REAL SIMPSN

REAL STEP 
REAL POLY $(8,201)$

REAL POLYDT $(8,201)$

REAL POLYDD $(8,201)$

REAL TTOMGA(201)

REAL TRPZOD

REAL $X$

REAL QPRIME $(8,8)$

REAL QTRANS $(8,8)$

REAL PRDCT1 $(8,8)$

REAL PRDCT2 $(8,8)$

REAL VALU(8)

REAL VECTR $1(8,8)$

REAL VECTR2 $(8,8)$

REAL VECTR3 $(8,8)$

$\begin{array}{ll}\text { INTEGER I } & \\ \text { INTEGER K } & \\ \text { INTEGER L } & \\ \text { INTEGER NP } & \\ \text { INTEGER NDIM } & \\ \text { INTEGER NDP } & \\ & \\ \text { INCLUDE } & \text { 'C: INCLUDE IBLADE. INC' } \\ \text { INCLUDE } & \text { 'C:INCLUDEVMODAL. INC' } \\ \text { INCLUDE } & \text { 'C:INCLUDEITURBN.INC' } \\ \text { INCLUDE } & \text { 'C:INCLUDEICONST2.INC' } \\ \text { INCLUDE } & \text { 'C:INCLUDEISPRING.INC' } \\ \text { INCLUDE } & \text { 'C:INCLUDE MMATRXI.INC' }\end{array}$

CHARACTER $* 50$ MODOUT

1100 FORMAT (A)

C Coordinate shape functions and their derivatives. These functions are polynomials that satisfy the boundary conditions for a cantilever blade, namely zero displacement and zero slope at the root. These functions will be used in linear combinations using a Rayleigh Ritz type procedure to determine the natural frequencies and modeshapes of the blade.

Teetering shape

CF1 $(X)=$ HUBRAD/(BLTIP+HUBRAD $) *(1 .-X)+X$

C Shape function for first symmetric bending

$\operatorname{CF} 2(X)=x * 2 *(x *(x-4.0)+6) /$.

CF2DOT $(X)=4 . * X *(X *(X-3)+3) /$.3 .

$\operatorname{CF2DD}(X)=4 . *(X *(X-2)+1$.

C Shape function for asymmetric bending and derivatives:

$\operatorname{CF} 3(X)=X * * 3 *(X *(3 . * X-10)+10) /$.

CF3DOT $(X)=5 . * X * * 2 *(X *(3 . * X-8)+6) /$.3 .

$\operatorname{CF3DD}(X)=20 . * x *(X *(3 . * X-6)+3) /$.3 .

C Shape function for second symmetric bending and derivatives:

$\operatorname{CF} 4(X)=X * 4 *(X *(2 . * X-6)+5$.

$\operatorname{CF4DOT}(X)=2 . * X * * 3 *(X *(6 . * x-15)+10$.

$\operatorname{CF} 4 D D(X)=60 . * X * 2 *(X *(X-2)+1$.

Extra shape function and it's derivatives:

CF5 $(X)=x * * 5 *(x *(10 . * x-28)+21) /$.3 .

CF5DOT $(X)=7 . \star X * * 4 *(X *(10 . * x-24)+15) /$.3 .

$\operatorname{CF5DD}(X)=14 . * X * * 3 *(X *(10 . * X-20)+10$.

C In the following set of polynomial shape function, every odd numbered array ( i.e. POLY $(1, I)$ ) corresponds to an asymetrical mode. Each even numbered menber corresponds to a symmetrical mode. There are three functions for each subspace, corresponding to a total dimension of $6 \times 6$ for the matrices, to solve for the modeshapes. Only the lowest two modes in each subspace are retained, i.e. two 
asymmetrical modes, and two symmetrical modes. All coefficient matrices passed on to module two will thus be of order $4 \times 4$.

Note that the first shape function for the asymmetical modes is the

rigid body teeter mode, whereas the first mode for the symmetrical modes is the polynomial CF2(x).

STEP $=1 . /(N P-1$.
BLTBLT $=$ BLTIP**2

$X=0$.

DO $110 I=1, N P$

$\operatorname{POLY}(1, I)=\operatorname{CF} 1(X) * \operatorname{CDELT3}$

POLYDT $(1,1)=$ CDELT3/(BLTIP+HUBRAD)

$\operatorname{POLYDD}(1,1)=0$.

$\operatorname{POLY}(2, I)=\operatorname{CF2}(X)$

$\operatorname{POLYDT}(2, I)=$ CF2DOT $(X) / B L T I P$

$\operatorname{POLYDD}(2, I)=\operatorname{CF2DD}(X) / B L T B L T$

$\operatorname{POLY}(3,1)=\operatorname{CF2}(X)$

$\operatorname{POLYDT}(3,1)=$ CF2DOT $(X) / B L T I P$

$\operatorname{POLYDD}(3,1)=\operatorname{CF2DD}(X) / B L T B L T$

$\operatorname{POLY}(4, I)=\operatorname{CF} 3(X)$

$\operatorname{POLYDT}(4, I)=\operatorname{CF} 3 D O T(X) / B L T I P$

$\operatorname{POLYDD}(4, I)=\operatorname{CF} 3 D D(X) / B L T B L T$

$\operatorname{POLY}(5, I)=\operatorname{CF} 3(X)$

POLYDT $(5, I)=\operatorname{CF3DOT}(X) / B L T I P$

$\operatorname{POLYDD}(5,1)=\operatorname{CF} 3 D D(X) / B L T B L T$

$\operatorname{POLY}(6, I)=\operatorname{CF} 4(X)$

$\operatorname{POLYDT}(6,1)=\operatorname{CF} 4 D O T(X) / B L T I P$

$\operatorname{POLYDD}(6,1)=\operatorname{CF} 4 D D(X) / B L T B L T$

$\operatorname{POLY}(7, I)=\operatorname{CF} 4(X)$

$\operatorname{POLYDT}(7,1)=$ CF4DOT $(X) / B L T I P$

$\operatorname{POLYDD}(7,1)=\operatorname{CF} 4 D D(X) / B L T B L T$

$\operatorname{POLY}(8, I)=\operatorname{CF5}(X)$

$\operatorname{POLYDT}(8, I)=$ CF5DOT $(X) / B L T I P$

$\operatorname{POLYDD}(8, I)=\operatorname{CF5DD}(X) / B L T B L T$

$X=X+$ STEP

110 CONTINUE

C Fill the intermediate function arrays which are used to

C compute the tension component integral for the

C centrifugal stiffening matrix.

$x=0$.

STEP = BLTIP /(NP - 1.)

DO $200 I=1, N P$

200 CONTINUE

$\operatorname{ETOMGA}(I)=\operatorname{MASS}(I) *($ HUBRAD $+\operatorname{STEP} *(I-1))$

C

Compute the tension component integral.

DO $300 I=1, N P$

TTOMGA(I) $=$ TRPZOD ( ETOMGA, I, NP, BLTIP) 300 CONTINUE

\footnotetext{
C Compute the coefficient matrices: ASUM, BSUM, CSUM.

C ASUM is the bending stiffness matrix, BSUM is the

C centrifugal stiffening matrix, and CSUM is the

C mass matrix.

DO $470 K=1,8$

DO $440 L=K, 8$
} 


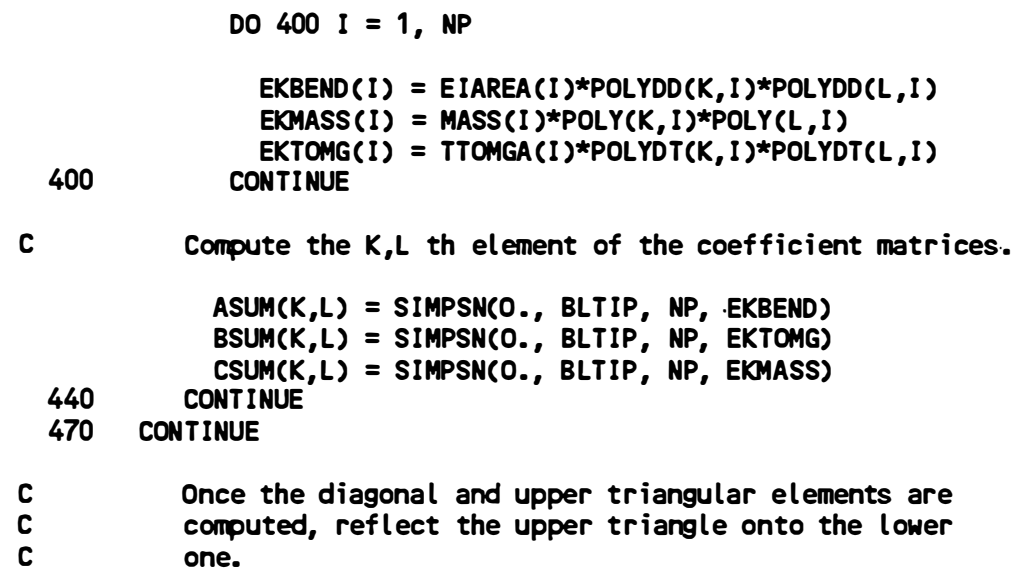

Once the diagonal and upper triangular elements are computed, reflect the upper triangle onto the lower one.

$\operatorname{ASUM}(1,2)=0$.

$\operatorname{ASUM}(1,4)=0$.

$\operatorname{ASUM}(1,6)=0$.

$\operatorname{ASUM}(1,8)=0$.

$\operatorname{ASUM}(2,3)=0$.

$\operatorname{ASUM}(2,5)=0$.

$\operatorname{ASUM}(2,7)=0$.

$\operatorname{ASUM}(3,4)=0$.

$\operatorname{ASUM}(3,6)=0$.

$\operatorname{ASUM}(3,8)=0$.

$\operatorname{ASUM}(4,5)=0$.

$\operatorname{ASUM}(4,7)=0$.

$\operatorname{ASUM}(5,6)=0$.

$\operatorname{ASUM}(5,8)=0$.

$\operatorname{ASUM}(6,7)=0$.

$\operatorname{ASUM}(7,8)=0$.

$\operatorname{BSUM}(1,2)=0$.

$\operatorname{BSUM}(1,4)=0$.

$\operatorname{BSUM}(1,6)=0$.

$\operatorname{BSUM}(1,8)=0$.

$\operatorname{BSUM}(2,3)=0$.

$\operatorname{BSUM}(2,5)=0$.

$\operatorname{BSUM}(2,7)=0$.

$\operatorname{BSUM}(3,4)=0$.

$\operatorname{BSUM}(3,6)=0$.

$\operatorname{BSUM}(3,8)=0$.

$\operatorname{BSUM}(4,5)=0$.

$\operatorname{BSUM}(4,7)=0$.

$\operatorname{BSUM}(5,6)=0$.

$\operatorname{BSUM}(5,8)=0$.

$\operatorname{BSUM}(6,7)=0$.

$\operatorname{BSUM}(7,8)=0$.

$\operatorname{CsUm}(1,2)=0$.

$\operatorname{CsUM}(1,4)=0$.

$\operatorname{csum}(1,6)=0$.

$\operatorname{CSUM}(1,8)=0$.

$\operatorname{CsUm}(2,3)=0$.

$\operatorname{CSUM}(2,5)=0$.

$\operatorname{CSUM}(2,7)=0$.

$\operatorname{CSUM}(3,4)=0$.

$\operatorname{CSUM}(3,6)=0$.

$\operatorname{CsUM}(3,8)=0$.

$\operatorname{CSUM}(4,5)=0$.

$\operatorname{CSUM}(4,7)=0$.

$\operatorname{CSUM}(5,6)=0$.

$\operatorname{CsUM}(5,8)=0$.

$\operatorname{CsUM}(6,7)=0$.

$\operatorname{csUm}(7,8)=0$.

DO $520 K=1,7$

DO $510 L=K+1,8$

$\operatorname{ASUM}(L, K)=\operatorname{ASUM}(K, L)$ 
$\operatorname{BSUM}(L, K)=\operatorname{BSUM}(K, L)$

$\operatorname{CSUM}(L, K)=\operatorname{CSUM}(K, L)$

510 CONTINUE

520 CONTINUE

NDIM $=8$

NDP $=8$

C Add the Centrifugal stiffening term to

C the bending stiffness terms.

C Form the new matrix DSUM

OMGA2 $=$ OMEGA $*$ OMEGA $* .010966227$

DO 1820 I $=1$, NDIM

DO $1830 \mathrm{~J}=1, \mathrm{NDIM}$

$\operatorname{DSUM}(I, J)=\operatorname{ASUM}(I, J)+\operatorname{OMGA2} * \operatorname{BSUM}(I, J)$

1830 CONTINUE

1820 CONTINUE

CALL JACOBI (CSUM,NDIM,VALU,VECTR1)

CALL EIGSRT (VALU,VECTR1,NDIM,NDP)

DO $880 I=1$, NDIM

DO $870 \mathrm{~J}=1$, NDIM

SVALU = SQRT(VALU $(J))$

870 CONTINUE

$\operatorname{QPRIME}(I, J)=\operatorname{VECTR} 1(I, J) / S V A L U$

880 CONTINUE

DO $900 I=1$,NDIM

DO $890 \mathrm{~J}=1$, NDIM

$\operatorname{QTRANS}(J, I)=\operatorname{QPRIME}(I, J)$

890 CONTINUE

900 CONTINUE

CALL MULT (QPRIME,DSUM,PRDCT1, NDIM,NDIM)

CALL MULT (PRDCT1,QTRANS,PRDCT2,NDIM,NDIM)

CALL JACOBI (PRDCT2,NDIM, VALU,VECTR2)

CALL EIGSRT(VALU,VECTR2,NDIM,NDP)

CALL MULT(VECTR2,QPRIME, VECTR3,NDIM,NDIM)

C The frequencies of interest are actually the square roots

C of the calculated eigenvalues.

DO $915 \mathrm{~J}=1$, NDIM

SUMSQ $=0$.

DO $910 I=1$, NDIM

SUMSQ $=$ SUMSQ $+\operatorname{VECTR3}(1, j) * \star 2$

910 CONTINUE

$\operatorname{MAG}(J)=$ SQRT(SUMSQ)

915 CONTINUE

DO $920 I=2$, NDIM

920 CONTINUE

FREQ(I) = SQRT( VALU(NDIM+1-I))

FREQSQ $=$ OMGA2+KO/(BLTIP**2*CSUM $(1,1))$

$\operatorname{FREQ}(1)=$ SQRT( FREQSQ)

DO $960 I=1$, NDIM

DO $940 \mathrm{~J}=1, \mathrm{NDIM}$

$\operatorname{LAMDA}(1, J)=\operatorname{VECTR} 3(1, \operatorname{NDIM}+1-\mathrm{J}) / \operatorname{MAG}(N D I M+1-J)$

940 CONTINUE

960 CONTINUE

PRINT*,'Enter name of modeshape and frequency file > ' READ 1100 , MODOUT

PRINT *, MODOUT 


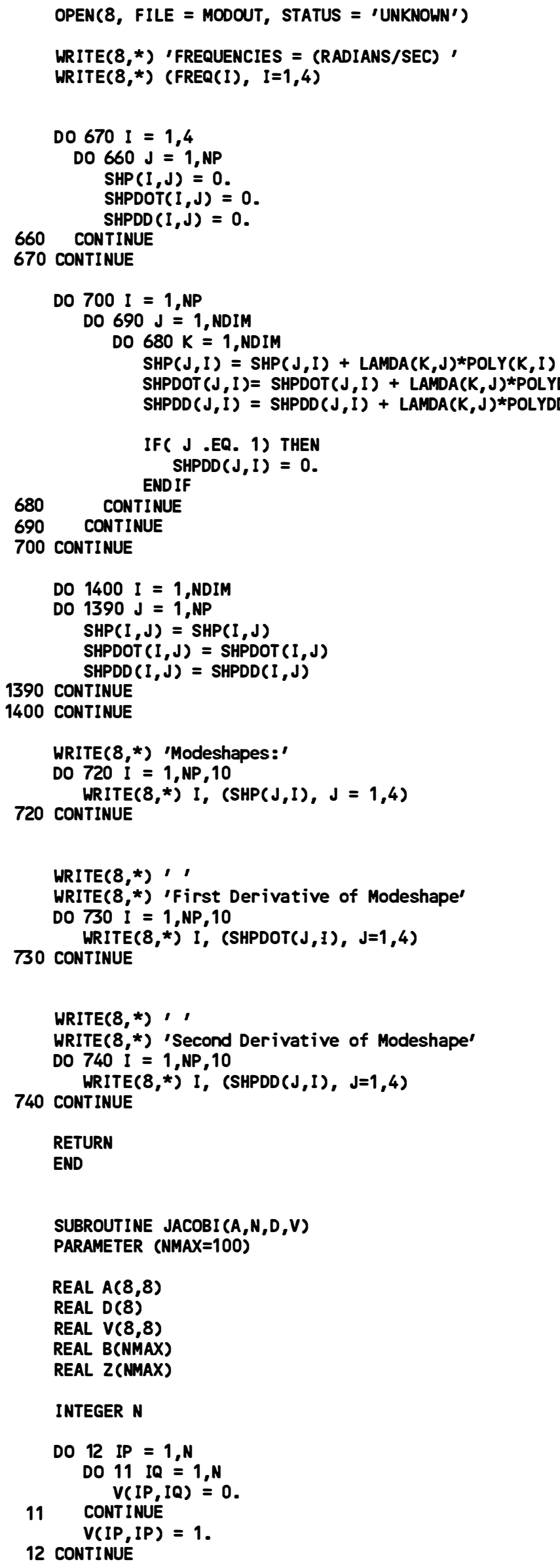

REAL $A(8,8)$

REAL D(8)

REAL V $(8,8)$

REAL B(NMAX)

REAL Z(NMAX)

INTEGER N

DO 12 IP $=1, \mathrm{~N}$

DO 11 IQ $=1, N$ $V(I P, I Q)=0$.

11 CONTINUE

$V(I P, I P)=1$.

12 CONTINUE 


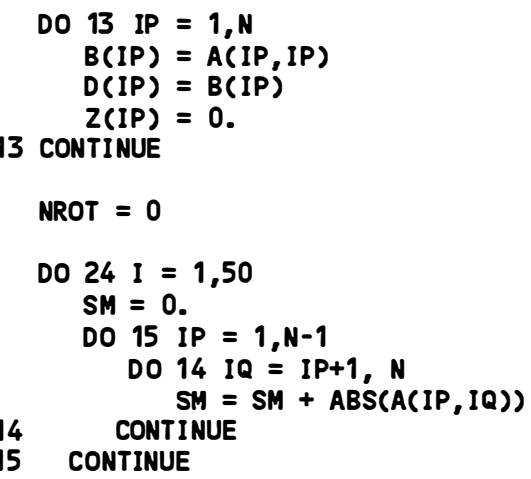

IF(SM .EQ. O.) RETURN

IF (I . LT . 4) THEN

ELSE

TRESH $=0.2 * S M / N * \star 2$

ENDIF

TRESH $=0$.

DO 22 IP $=1, \mathrm{~N}-1$

DO 21 IQ $=I P+1, N$

$G=100 . \star A B S(A(I P, I Q))$

IF( G .LT. 1.0E-08)THEN

$A(I P, I Q)=0$.

ELSEIF( ABS(A(IP, IQ)) .GT. TRESH)THEN

$H=D(I Q)-D(I P)$

$I F(A B S(H)+G$.EQ. ABS(H))THEN

$T=A(I P, I Q) / H$

ELSE

THETA $=0.5 * H / A(I P, I Q)$

$T=1 . /(A B S(T H E T A)+\operatorname{SQRT}(1 .+$ THETA**2))

IF(THETA .LT. 0.) $T=-1 . * T$

ENDIF

$C=1 . / \operatorname{SQRT}(1+T * * 2)$

$S=T * C$

$T A U=S /(1 .+C)$

$H=T * A(I P, I Q)$

$Z(I P)=Z(I P)-H$

$Z(I Q)=Z(I Q)+H$

$D(I P)=D(I P)-H$

$D(I Q)=D(I Q)+H$

$A(I P, I Q)=0$.

DO $16 \mathrm{~J}=1, \mathrm{IP}-1$

$G=A(J, I P)$

$H=A(J, I Q)$

$A(J, I P)=G-S *(H+G * T A U)$

16

$A(J, I Q)=H+S^{*}\left(G-H^{*} T A U\right)$

CONTINUE

DO $17 \mathrm{~J}=I P+1, I Q-1$

$G=A(I P, J)$

$H=A(J, I Q)$

$A(I P, J)=G-S *(H+G * T A U)$

$A(J, I Q)=H+S^{*}\left(G-H^{*} T A U\right)$

17 CONT INUE

DO $18 \mathrm{~J}=\mathrm{IQ+1}, \mathrm{N}$

$G=A(I P, J)$

$H=A(I Q, J)$

$A(I P, J)=G-S *(H+G * T A U)$

$A(I Q, J)=H+S^{*}(G-H * T A U)$

18 CONTINUE

DO $19 \mathrm{~J}=1, \mathrm{~N}$

$G=V(J, I P)$

$H=V(J, I Q)$

$V(J, I P)=G-S *(H+G * T A U)$

19

$V(J, I Q)=H+S *(G-H * T A U)$

CONTINUE

ENDIF

NROT $=$ NROT +1 

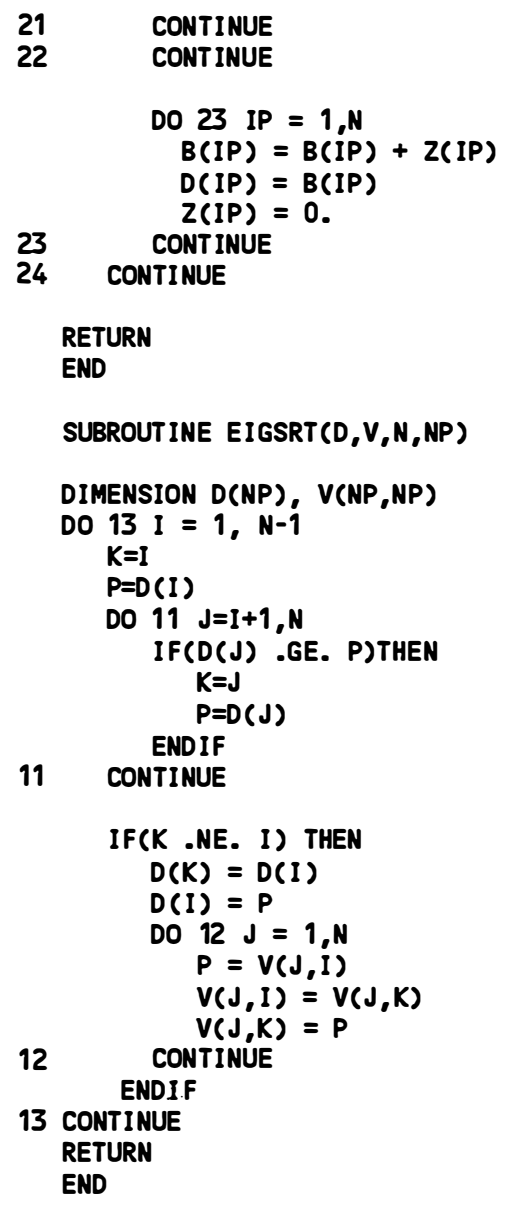

SUBROUTINE INPUT ( NP , NPTS , FILOUT )

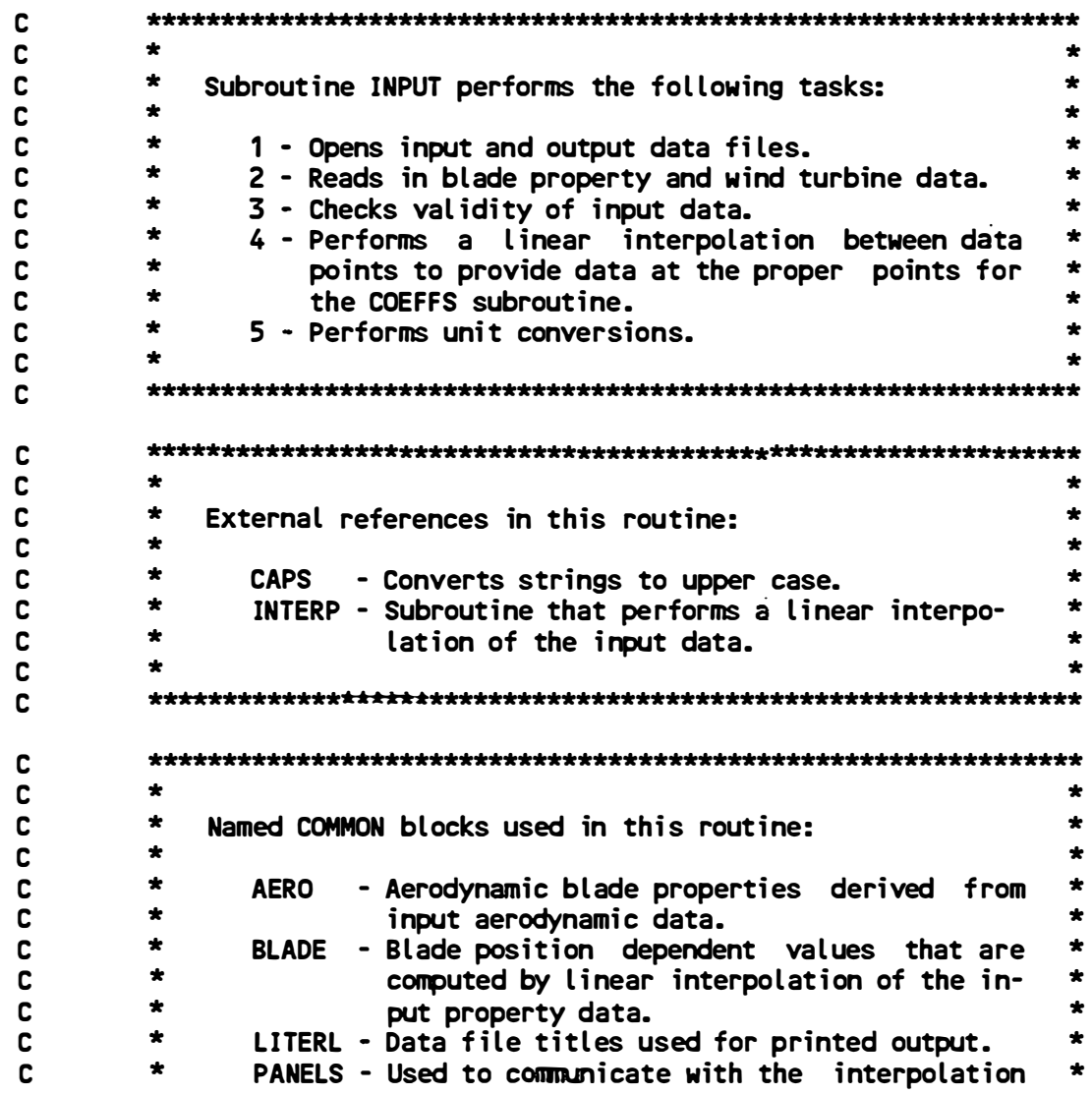




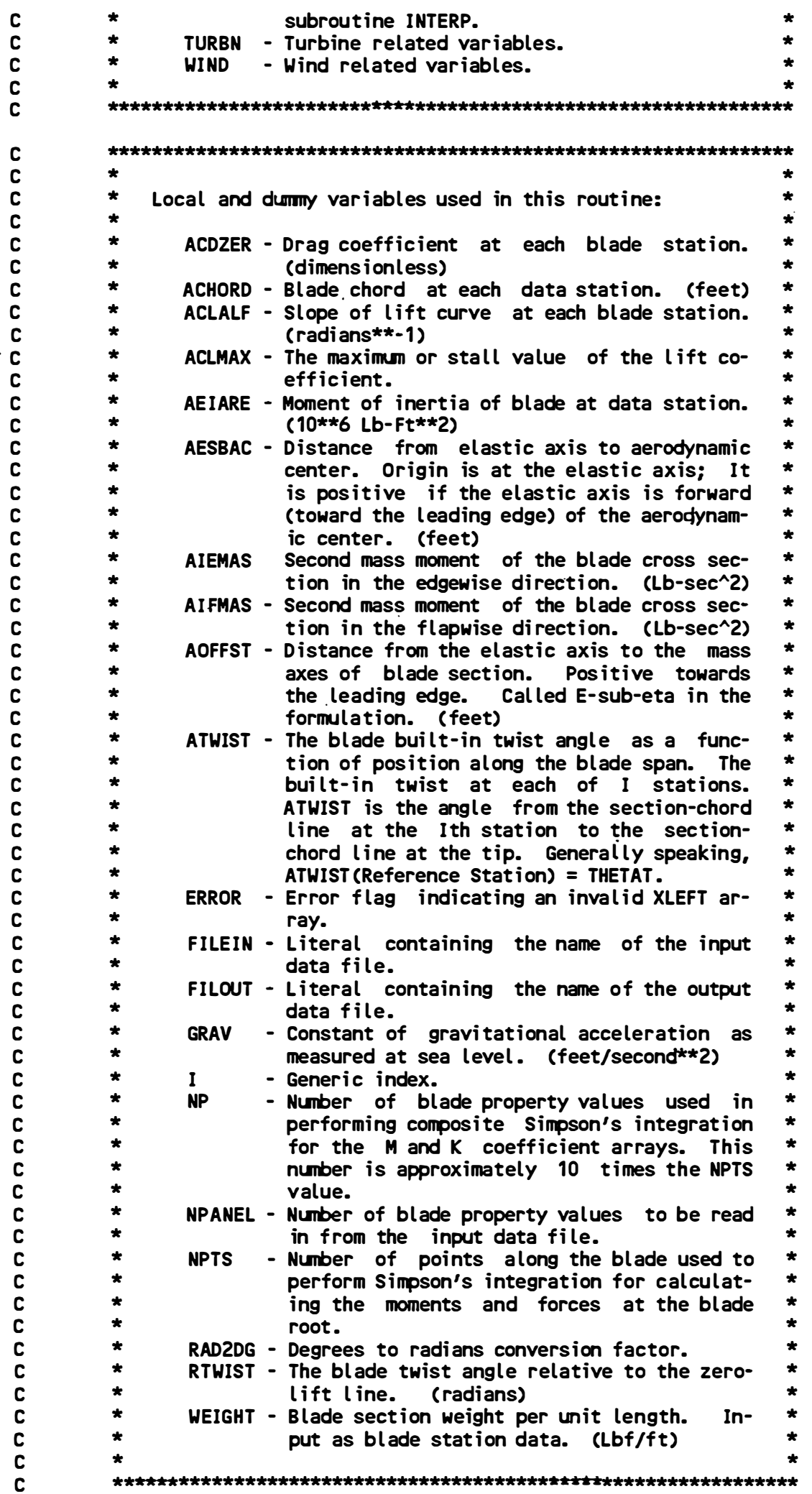

$\begin{array}{ll}\text { REAL } & \text { ACDZER (11) } \\ \text { REAL } & \text { ACHORD (11) } \\ \text { REAL } & \text { ACLALF (11) } \\ \text { REAL } & \text { ACLMAX (11) } \\ \text { REAL } & \text { AEIARE (11) } \\ \text { REAL } & \text { AESBAC (11) } \\ \text { REAL } & \text { AIEMAS (11) } \\ \text { REAL } & \text { AIFMAS (11) } \\ \text { REAL } & \text { AOFFST (11) } \\ \text { REAL } & \text { ATWIST (11) }\end{array}$




\begin{tabular}{|c|c|}
\hline $\begin{array}{l}\text { REAL } \\
\text { REAL } \\
\text { REAL } \\
\text { REAL }\end{array}$ & $\begin{array}{l}\text { GRAV } \\
\text { RAD2DG } \\
\text { RTWIST (11) } \\
\text { WEIGHT (11) }\end{array}$ \\
\hline $\begin{array}{l}\text { INTEGER } \\
\text { INTEGER } \\
\text { INTEGER } \\
\text { INTEGER }\end{array}$ & $\begin{array}{l}\text { I } \\
\text { NP } \\
\text { NPANEL } \\
\text { NPTS }\end{array}$ \\
\hline LOGICAL & ERROR \\
\hline $\begin{array}{l}\text { CHARACTER } * 50 \\
\text { CHARACTER } * 50\end{array}$ & $\begin{array}{l}\text { FILEIN } \\
\text { FILOUT }\end{array}$ \\
\hline INCLUDE & 'C:INCLUDE \\
\hline INCLUDE & 'C:INCLUDE \BLADE.INC' \\
\hline INCLUDE & 'C: INCLUDE ILITERL.INC' \\
\hline INCLUDE & 'C:INCLUDEXPANELS.INC' \\
\hline INCLUDE & 'C:INCLUDE \TURBN.INC' \\
\hline INCLUDE & 'C:INCLUDE ITRBINF.INC' \\
\hline INCLUDE & 'C:INCLUDEIWIND.INC' \\
\hline INCLUDE & 'C:INCLUDE IHUBPRP. INC' \\
\hline INCLUDE & 'C:INCLUDE SSPRING.INC' \\
\hline $\begin{array}{l}\text { DATA } \\
\text { DATA }\end{array}$ & $\begin{array}{ll}\text { GRAV / } 32.1740 / \\
\text { RAD2DG / } 57.29577951308233 /\end{array}$ \\
\hline
\end{tabular}

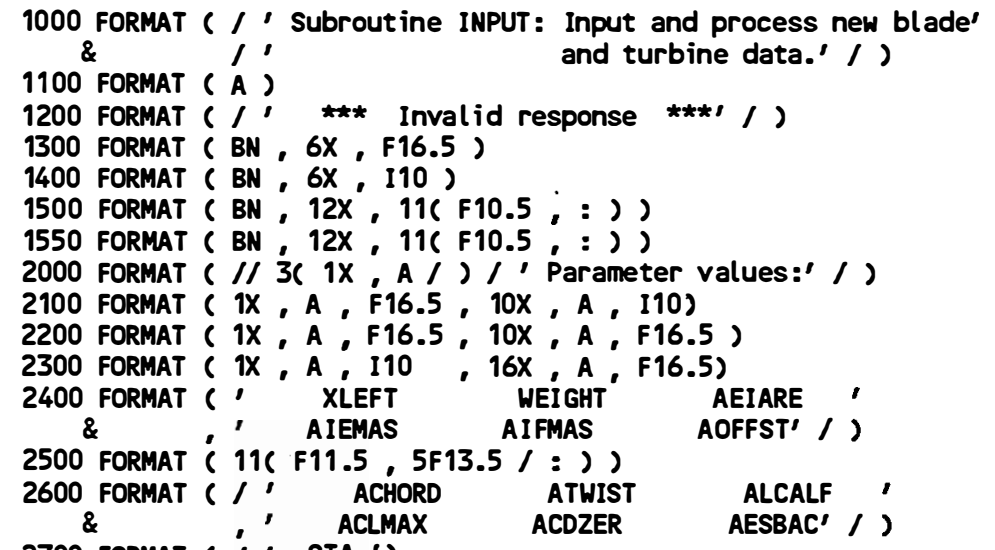


C Get input and output data file names. Open the input file.

C Determine rotor type. Read data from input file.

PRINT 1000

PRINT *, 'Enter name of input data file > '

READ 1100, FILEIN

PRINT *, FILEIN

OPEN ( 1 , FILE=FILEIN , STATUS=' OLD' $\left.^{\prime}\right)$

PRINT * $\quad 1$,

PRINT *', 'Enter name of output data file > '

READ 1100, FILOUT

PRINT *, FILOUT

CALL CAPS ( FILOUT )

110 READ $(1,1100)$ TITLE1

READ $(1,1100)$ TITLE2

READ $(1,1100)$ TITLE3

READ (1,1300) ALENTH

READ $(1,1300)$ ALPHAO

READ $(1,1300)$ BETAO

READ $(1,1300)$ BLSHNK

READ $(1,1300)$ BLTIP

READ $(1,1300)$ CHI

READ $(1,1300)$ CSUBMA

READ $(1,1300)$ DRGFRM

READ $(1,1300)$ HUBHT

READ $(1,1300)$ HUBRAD

READ (1,1400) KSHADW

READ $(1,1400)$ NBLADS

READ $(1,1400)$ NPANEL

READ $(1,1300)$ OMEGA

READ $(1,1300)$ PHIAMP

READ $(1,1300)$ PHIOMG

READ $(1,1300)$ PHIO

READ $(1,1300)$ PSIZER

READ $(1,1300)$ SHERXP

READ $(1,1300)$ THETAP

READ $(1,1300)$ THETAT

READ $(1,1300)$ TSUBP

READ $(1,1300)$ TSUBO

READ $(1,1300)$ HUBMAS

READ $(1,1300)$ UNDSLG

READ $(1,1300)$ HUBDIS

READ $(1,1300)$ VHUB

READ $(1,1300)$ DELT3

READ $(1,1300)$ BETA1

READ $(1,1300)$ BETA2

READ $(1,1300)$ KO

READ $(1,1300) \cdot K 1$

READ $(1,1300) \quad K 2$

READ $(1,1300)$ CDAMP

READ ( 1,1500$)$ '( XLEFT (I), I=1,NPANEL )

READ $(1,1500)$ ( WEIGHT(I)，I=1,NPANEL )

$\operatorname{READ}(1,1500)$ ( $\operatorname{AEIARE}(1), I=1$,NPANEL )

$\operatorname{READ}(1,1500)$ ( AIEMAS(I), I=1,NPANEL )

$\operatorname{READ}(1,1500)$ ( AIFMAS(I), I=1,NPANEL )

READ $(1,1500)$ ( AOFFST(I) I I 1 ,NPANEL )

READ $(1,1500)$ ( $A C H O R D(I), I=1$,NPANEL )

READ $(1,1500)$ ( ATWIST(I) , I=1,NPANEL )

$\operatorname{READ}(1,1500)$ ( $\operatorname{ACLALF}(I), I=1$,NPANEL )

$\operatorname{READ}(1,1500)$ ( $\operatorname{ACLMAX}(I), I=1, N P A N E L)$

$\operatorname{READ}(1,1500)$ ( $\operatorname{ACDZER}(I), I=1$,NPANEL )

$\operatorname{READ}(1,1500)$ ( $\operatorname{AESBAC}(I), I=1, N P A N E L)$

READ $(1,1400)$ NUMSCN

READ $(1,1300)$ TIMINC

READ $(1,1400)$ MSTAT

$\operatorname{READ}(1,1550)^{\circ}$ ( $\left.\operatorname{STA}(I), I=1, \operatorname{MSTAT}\right)$ 
CLOSE( 1 )

C

Echo input data.

PRINT 2000, TITLE1 , TITLE2 , TITLE3

PRINT 2100, 'ALENTH $=1$, ALENTH , 'NPANEL $=1$, NPANEL

PRINT 2200, 'ALPHAO $=$, , ALPHAO , 'OMEGA $=1$, OMEGA

PRINT 2200, 'BETAO $=1$, BETAO, 'PHIO $=1$, PHIO

PRINT 2200, 'BLSHNK $=$ ', BLSHNK,' 'PHIAMP $=$ ', PHIAMP

PRINT 2200, 'BLTIP $=1$, BLTIP, 'PHIOMG $=1$, PHIOMG

PRINT 2200, 'CHI = ', CHI ',PSIZER = ', PSIZER

PRINT 2200, 'CSUBMA $=1$, CSUBMA, 'SHERXP $=1$, SHERXP

PRINT 2200, 'DRGFRM $=$ ', DRGFRM , 'THETAP $=$, , THETAP

PRINT 2200, 'HUBHT $=$ ', HUBHT , 'THETAT $=1$, THETAT

PRINT 2200, 'HUBRAD $=$ ', HUBRAD, 'TSUBO $=$ ', TSUBO

PRINT 2300, 'KSHADW $=$, KSHADW, 'TSUBP $=$ ', TSUBP

PRINT 2300, 'NBLADS $=1$, NBLADS, 'VHUB $=1$, VHUB

PRINT 2200, 'HUBMAS $=1$, HUBMAS, 'UNDSLG $=1$, UNDSLG

PRINT 2200, 'HUBDIS $=1$, HUBDIS, 'DELTA3 $=\prime$, DELT3

PRINT 2200, 'BETA1 =', BETA1, 'BETA2 = ', BETA2

PRINT 2200, 'KO =', KO , 'K1 $K 1=$ ', K1

PRINT 2200, 'K2 $=1, K 2$

PRINT 2200, 'CDAMP $=1$, CDAMP

PRINT 2300, 'NUMSCN $=1$, NUMSCN

PRINT 2200, 'TIMINC $=$,', TIMINC

PRINT 2300, 'MSTAT $=1$, MSTAT

PRINT * , ,

PRINT *, 'Hit <Enter> to continue...'

200 PRINT 2400

PRINT 2500, ( XLEFT(I) , WEIGHT(I) , AEIARE(I) , AIEMAS(I)

\&

, AIFMAS(I) , AOFFST(I) , I=1,NPANEL )

PRINT *,, 1

PRINT *, 'Hit <Enter> to continue...'

210 PRINT 2600

\&

PRINT 2500, ( ACHORD(I), ATWIST(I) , ACLALF(I), ACLMAX (I)

, ACDZER(I) , AESBAC(I) , I=1,NPANEL )

PRINT *, , ,

PRINT *, 'Hit <Enter> to continue...'

220 PRINT 2700

PRINT 2750, ( STA(I), I=1,MSTAT)

PRINT * , 1

PRINT *', 'Hit <Enter> to continue...'

C Check validity of XLEFT array. XLEFT must start at blade root

C and end at blade tip.

300 ERROR $=$.FALSE.

IF ( XLEFT(1) .NE. 0.0 ) THEN

PRINT 3000, XLEFT(1)

ERROR = .TRUE.

END IF

IF ( XLEFT(NPANEL) . NE. BLTIP ) THEN PRINT 3100, NPANEL , XLEFT(NPANEL)， BLTIP ERROR $=$.TRUE.

END IF

IF ( ERROR ) THEN PRINT 3200 STOP

END IF

Perform unit conversions - Lbm to slugs, degrees to radians. 
DO $400 \mathrm{I}=1$, NPANEL

WEIGHT(I) = WEIGHT(I)/GRAV

$\operatorname{AEIARE}(I)=\operatorname{AEIARE}(I) * 1 . E 06$

$\operatorname{AIEMAS}(I)=\operatorname{AIEMAS}(I)$

$\operatorname{AIFMAS}(I)=\operatorname{AIFMAS}(I)$

400 CONTINUE

RTWIST(I) = ( THETAT - ATWIST(I) - ALPHAO )/RAD2DG

C

Calculate the cosine, sine, and tangent of the delta-3 angle

DELT3 $=(3.141593 / 180)$.$* DELT3$

CDELT3 $=$ COS(DELT3)

SDELT3 = SIN(DELT3)

TDELT3 = TAN(DELT3)

C Perform linear interpolation of blade properties to set up arrays of blade property data at equidistant points along the blade. The arrays are used by the COEFFs subroutine to produce the coefficient matricies.

STEP $=$ BLTIP/( NP - 1 )

CALL INTERP ( WEIGHT, MASS , NPANEL , NP )

CALL INTERP ( AIEMAS , IEMASS , NPANEL , NP )

CALL INTERP ( AIFMAS , IFMASS , NPANEL , NP )

CALL INTERP ( AEIARE , EIAREA, NPANEL, NP )

CALL INTERP ( AOFFST, OFFSET , NPANEL , NP )

CALL INTERP ( ACHORD, CHORD, NPANEL, NP )

CALL INTERP ( RTWIST; THETAO， NPANEL，NP )

C These property arrays will be used by the RUN subroutine for

C solving the governing equations and for calculation of the loads and moments.

STEP = BLTIP/( NPTS - 1 )

CALL INTERP ( ACLALF , CLALFA , NPANEL , NPTS )

CALL INTERP ( ACDZER , CDZERO , NPANEL , NPTS )

CALL INTERP ( AESBAC , ESUBAC , NPANEL , NPTS )

CALL INTERP ( ACLMAX; CLMAX; NPANEL ; NPTS )

RETURN

END

SUBROUTINE INTERP ( GIVEN , CMPUTD， NPANEL , NP ) 


$\begin{array}{ll}\text { INTEGER } & \text { NP } \\ \text { INTEGER } & \text { NPANEL } \\ \text { REAL } & \text { CMPUTD (NP) } \\ \text { REAL } & \text { GIVEN (NPANEL) } \\ \text { REAL } & \text { PTR } \\ \text { REAL } & \text { SLOPE } \\ \text { INTEGER } & \text { IPNL } \\ \text { INTEGER } & \text { JPT } \\ \text { LOGICAL } & \text { MOVE } \\ \text { INCLUDE } & \text { 'C:INCLUDEIPANELS.INC' }\end{array}$

C Initialize GIVEN index and pointer.

IPNL $=1$

PTR $=0.0$

C Compute slope between first two data points.

SLOPE $=($ GIVEN(2) - GIVEN(1) )/XLEFT(2)

C Compute property at each of the NP evenly spaced points.

DO 20 JPT=1,NP-1

CMPUTD(JPT) $=\operatorname{GIVEN}(I P N L)+\operatorname{SLOPE}($ PTR - XLEFT(IPNL) )

$P T R=P T R$ + STEP

C Make sure that the new data point is inside the current

C panel. Otherwise, move over one step.

IF ( JPT .LT. NP-1) THEN

MOVE $=$.FALSE.

10 IF ( PTR . GT. XLEFT(IPNL+1) ) THEN IPNL = IPNL +1 MOVE $=$.TRUE. GO TO 10

END IF

\&

IF ( MOVE ) SLOPE $=($ GIVEN(IPNL+1) - GIVEN(IPNL) )

$($ XLEFT(IPNL+1) - XLEFT(IPNL) )

END IF

20 CONTINUE

CMPUTD (NP) = GIVEN (NPANEL)

RETURN

END

FUNCTION LNTH ( STRING )

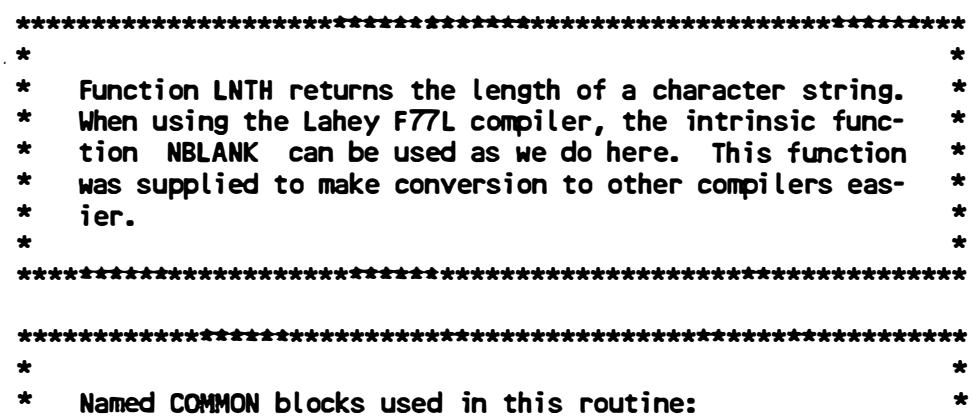




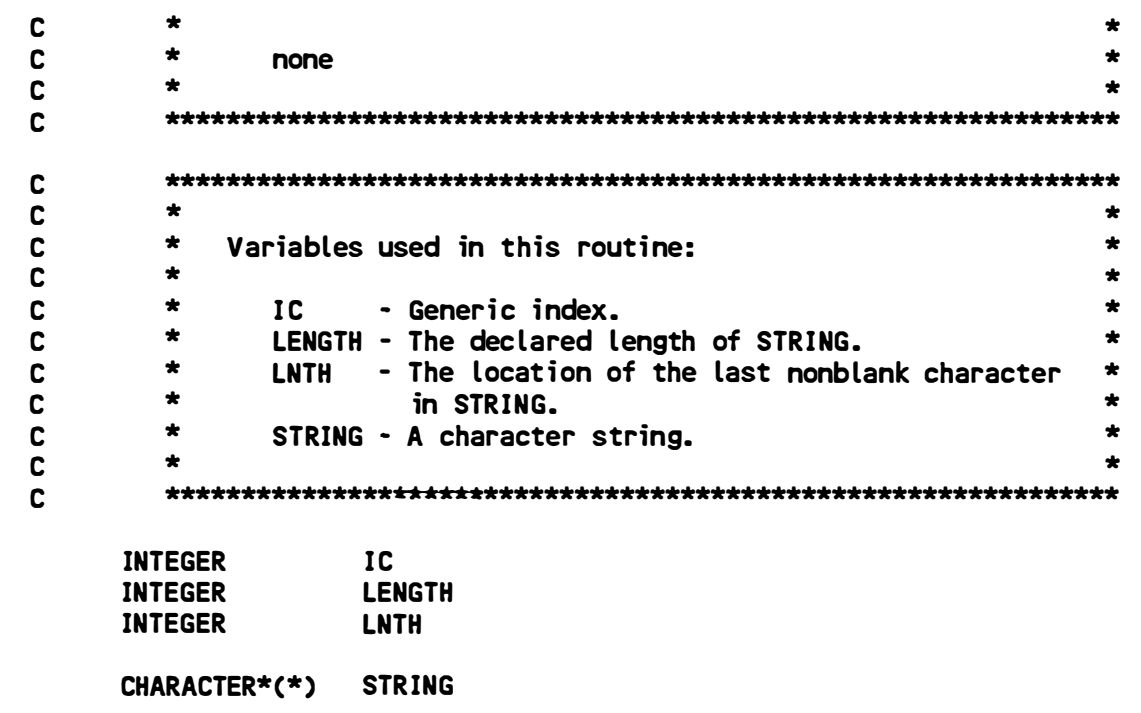

C Get the declared length of STRING using the FORTRAN 77 intrinsic C function LEN.

LENGTH = LEN( STRING )

C Find the location of the last nonblank character in STRING.

DO 100 IC=LENGTH, 1,-1

LNTH = IC

IF ( STRING(IC:IC) .NE. ' ' ) GO TO 200

100 CONTINUE

C STRING is all blanks.

LNTH $=0$

200 RETURN

END

SUBROUTINE PRNCOE ( FILOUT, NPTS )

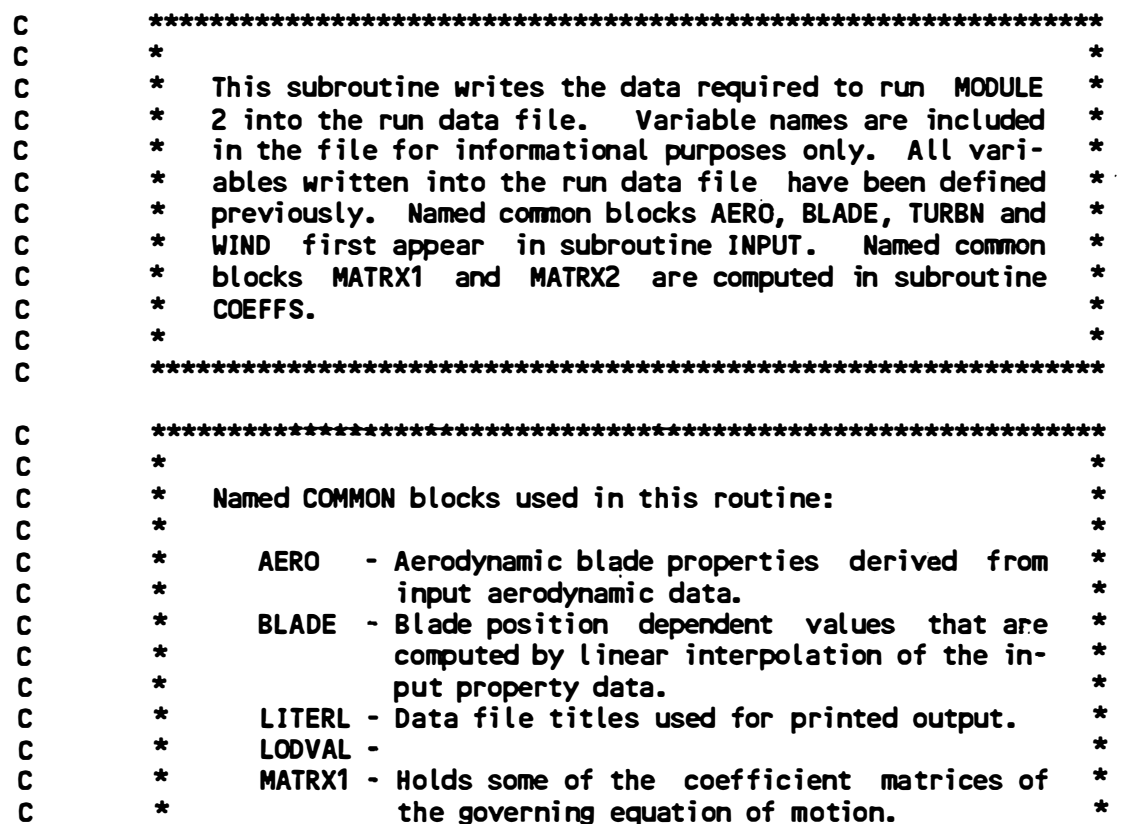




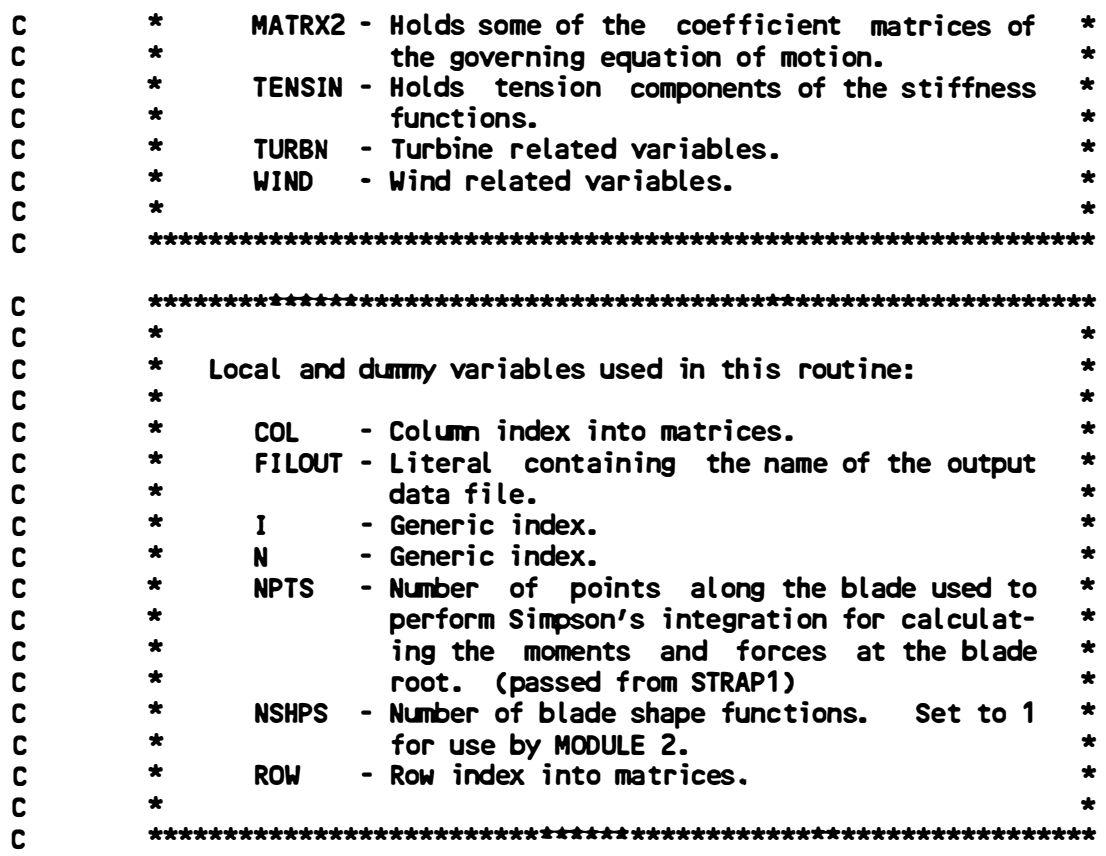

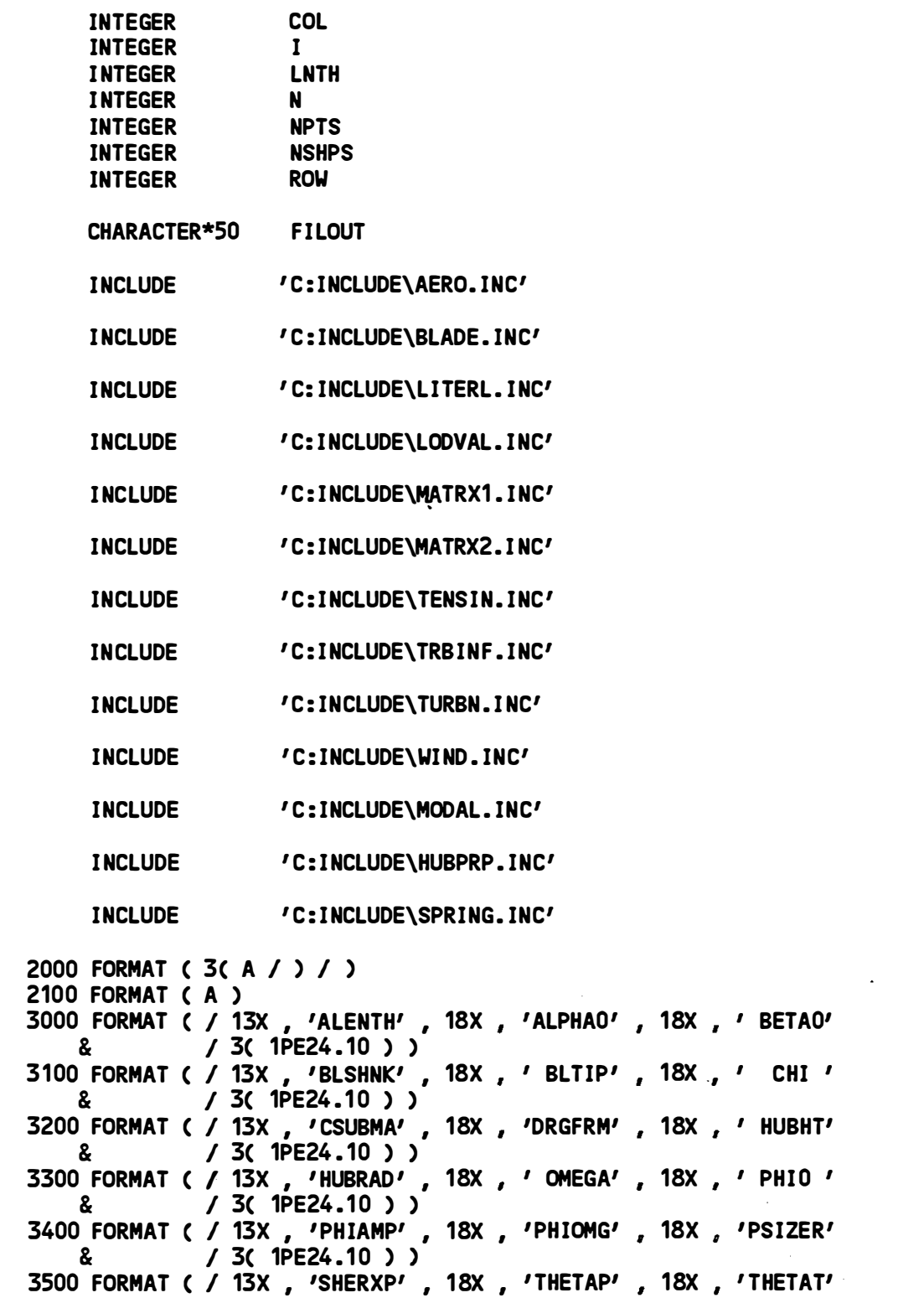




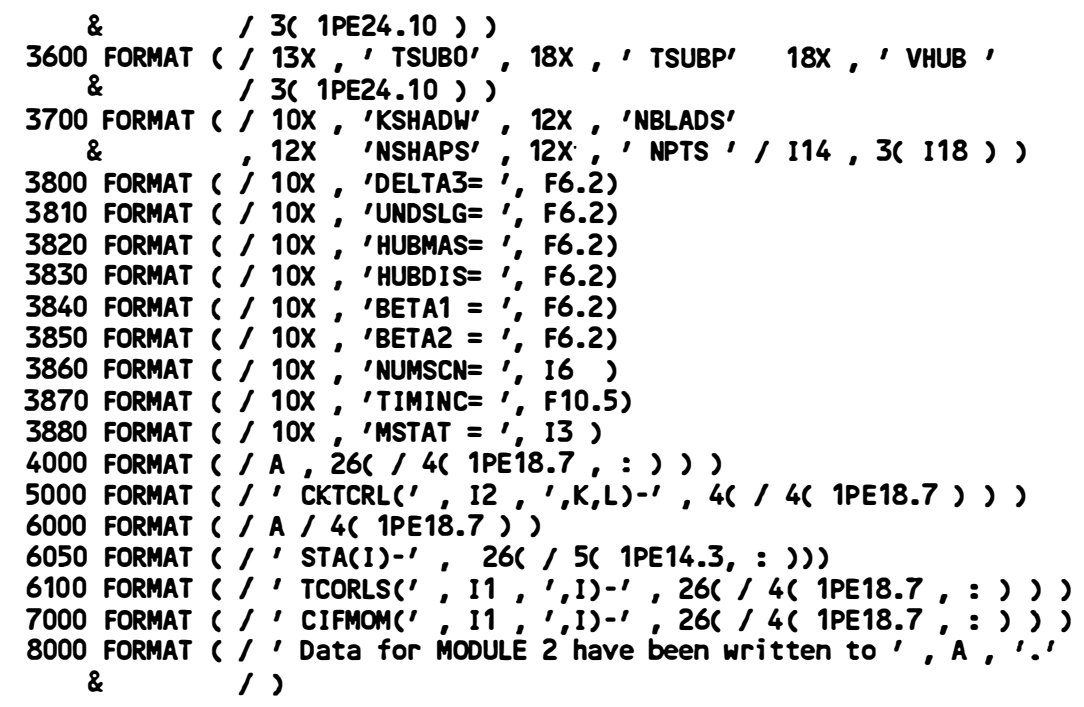

C Open output data file.

OPEN ( 2 , FILE=FILOUT , STATUS='UNKNOWN' )

C Put titles and hub type in run data file.

WRITE (2,2000) TITLE1， TITLE2， TITLE3

C

Put scalar values into run data file.

$\begin{array}{ll}\text { WRITE }(2,3000) & \text { ALENTH , ALPHAO , BETAO } \\ \text { WRITE }(2,3100) & \text { BLSHNK , BLTIP , CHI } \\ \text { WRITE }(2,3200) & \text { CSUBMA , DRGFRM , HUBHT } \\ \text { WRITE }(2,3300) & \text { HUBRAD , OMEGA PHIO } \\ \text { WRITE }(2,3400) & \text { PHIAMP , PHIOMG , PSIZER } \\ \text { WRITE }(2,3500) & \text { SHERXP , THETAP , THETAT } \\ \text { WRITE }(2,3600) & \text { TSUBO , TSUBP , VHUB }\end{array}$
MODULE 2. Use the variable name NSHPS to avoid overwriting NSHAPS.

NSHPS $=1$

WRITE (2,3700) KSHADW , NBLADS , NSHPS , NPTS

DELT3 $=$ DELT3*180./3.14159

WRITE $(2,3800)$ DELT3

WRITE $(2,3810)$ UNDSLG

WRITE $(2,3820)$ HUBMAS

WRITE $(2,3830)$ HUBDIS

WRITE $(2,3840)$ BETA1

WRITE $(2,3850)$ BETA2

Put turbulence calculation info into run data file.

WRITE $(2,3860)$ NUMSCN

WRITE $(2,3870)$ TIMINC

WRITE $(2,3880)$ MSTAT

WRITE $(2,6050) \quad(\operatorname{STA}(I), I=1$, MSTAT)

Put vector values into run data file.

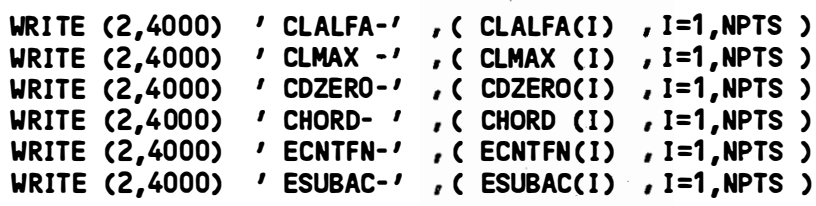


WRITE (2,4000) , THETAO-1, ( THETAO(I) , I=1,NPTS )

Put matrix coefficient values into run data file.

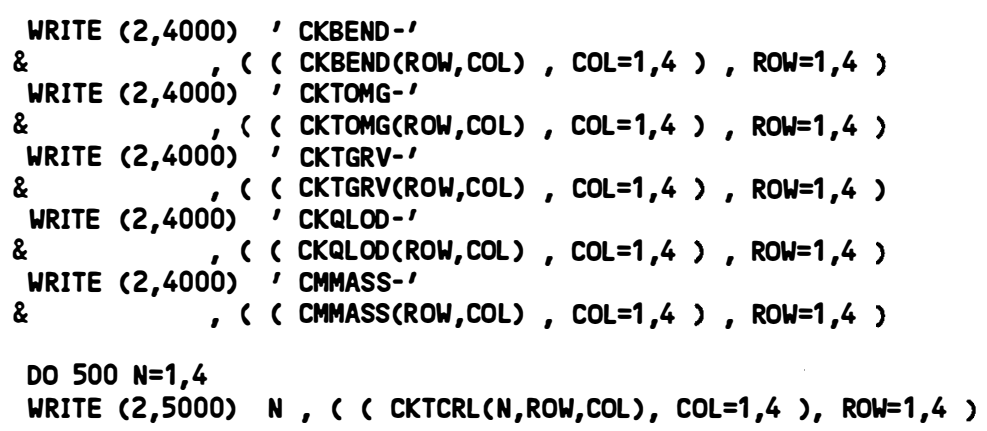

C Put vector coefficient values into run data file.

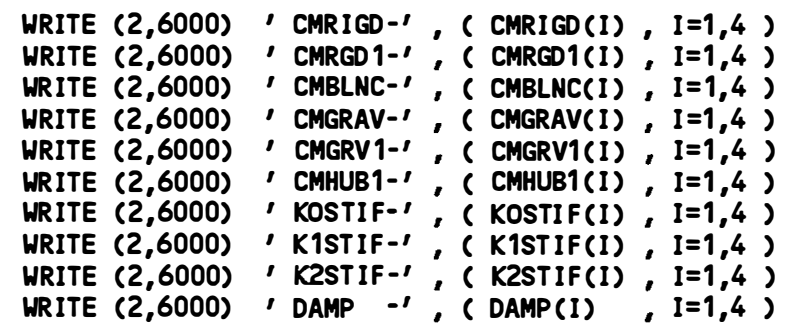

C Put matrix tension values into run data file.

DO $600 \mathrm{~N}=1,4$

600 WRITE $(2,6100) N,(\operatorname{TCORLS}(N, I), I=1, N P T S)$

C Put vector gravity and centrifugal stiffening values into run

C data file.

WRITE $(2,4000)$, TGRAV-1, ( TGRAV(I), I=1,NPTS )

WRITE $(2,4000)$, TOMGA-1; ( TOMGA(I); I=1,NPTS )

C Put property values into run data file.

DO $700 \mathrm{~N}=1,4$

700 WRITE $(2,7000) N,(\operatorname{CIFMOM}(N, 1), I=1$, NPTS $)$

WRITE $(2,4000)$, DELTIM-1 , ( DELTIM(I), I=1,NPTS )

WRITE $(2,4000)$, OFFMAS-1, ( OFFMAS(I): I=1,NPTS )

DO $710 I=1,201,10$

710 CONTINUE

$\operatorname{WRITE}(2, *)(\operatorname{SHP}(J, I), J=1,4)$

DO $720 I=1,201,10$

720 CONTINUE

$\operatorname{WRITE}(2, *)$ (SHPDOT $(J, I), J=1,4)$

DO $730 I=1,201,10$ 730 CONTINUE

$\operatorname{WRITE}(2, *)(\operatorname{SHPDD}(J, I), J=1,4)$

C Run file generation complete. Print message and close file.

WRITE $(*, 8000)$ FILOUT(1:LNTH(FILOUT))

CLOSE ( 2 )

RETURN

END

FUNCTION SIMPSN ( LOWLIM , UPLIM , NPTS , FOFX ) 


$\begin{array}{ll}\text { REAL } & \text { FOFX (201) } \\ \text { REAL } & \text { H } \\ \text { REAL } & \text { LOWLIM } \\ \text { REAL } & \text { SIMPSN } \\ \text { REAL } & \text { UPLIM } \\ & \\ \text { INTEGER } & \text { I } \\ \text { INTEGER } & \text { NPTS }\end{array}$

H $=($ UPLIM - LOWLIM )/( NPTS - 1 )

SIMPSN $=0.0$

Add in the intermediate points. In the formulation, all even numbered points have coefficient of 4. In this case, the index must be shifted to form the proper coefficient.

DO $10 I=2$, NPTS $-1,2$

10 SIMPSN $=$ SIMPSN $+4.0 *$ FOFX $(I)+2.0 *$ FOFX $(I+1)$

SIMPSN $=$ SIMPSN + FOFX(1) - FOFX(NPTS $)$

SIMPSN $=H *$ SIMPSN $/ 3.0$

RETURN

END

FUNCTION TRPZOD ( FOF , LOWLIM , NP , BLTIP ) 


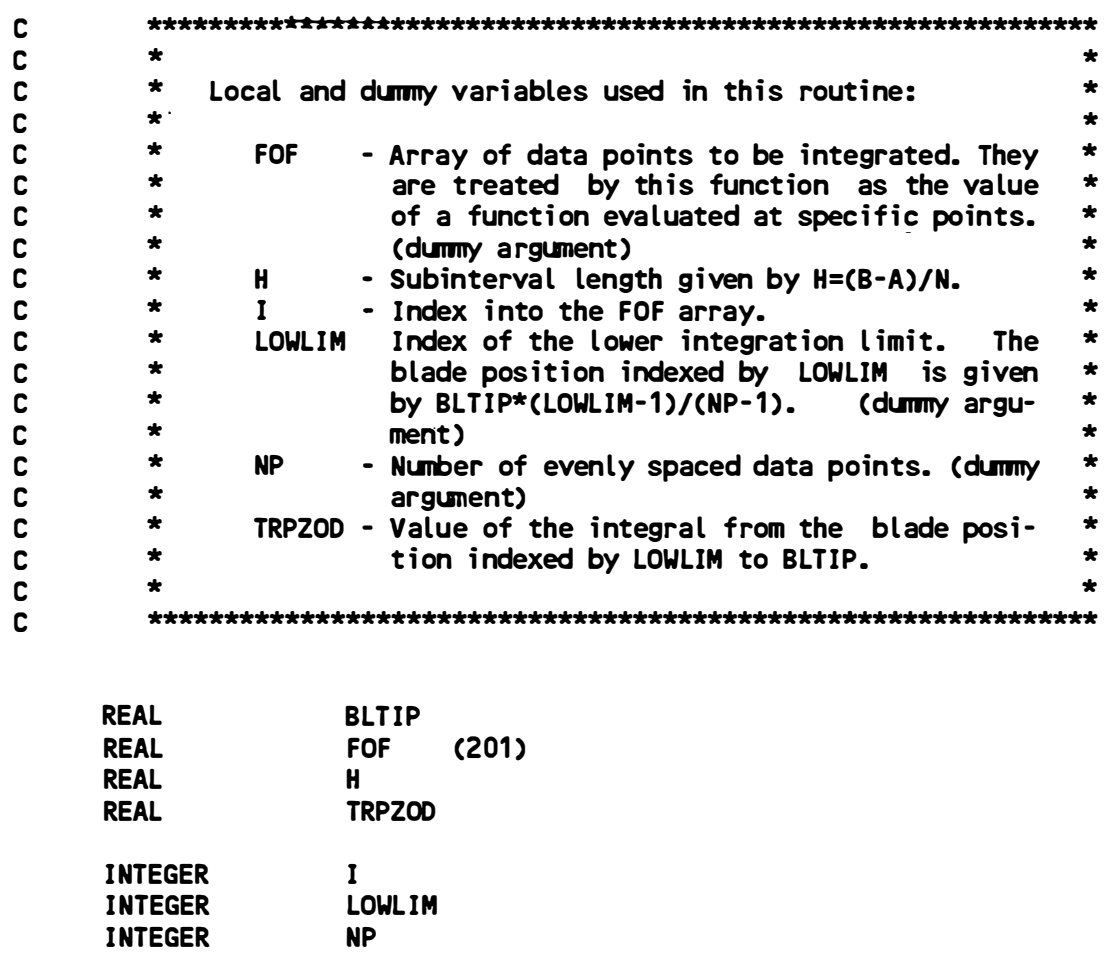

C Check to see if the lower and upper limits of integration are C. the same. If so, the integral is zero.

IF ( LOWLIM .EQ. NP ) THEN

TRPZOD $=0.0$

RETURN

END IF

C Compute the distance between data points.

$H=B L T I P /(N P-1)$

C

Initialize integral to the contribution of the end points.

$T R P Z O D=0.5 *(F O F(L O W L I M)+F O F(N P))$

C

If there are only two data points, then we are done.

IF ( LOWLIM+1 .EQ. NP ) GO TO 20

C

Add in the contribution of the intermediate points.

DO $10 I=$ LOWLIM+1, NP-1

10 TRPZOD $=$ TRPZOD + FOF $(I)$

C

Multiply by the interval width and return.

20 TRPZOD $=H * T R P Z O D$

RETURN

END

SUBROUTINE MULT ( AMATRX , BMATRX, RESULT, M , N ) 


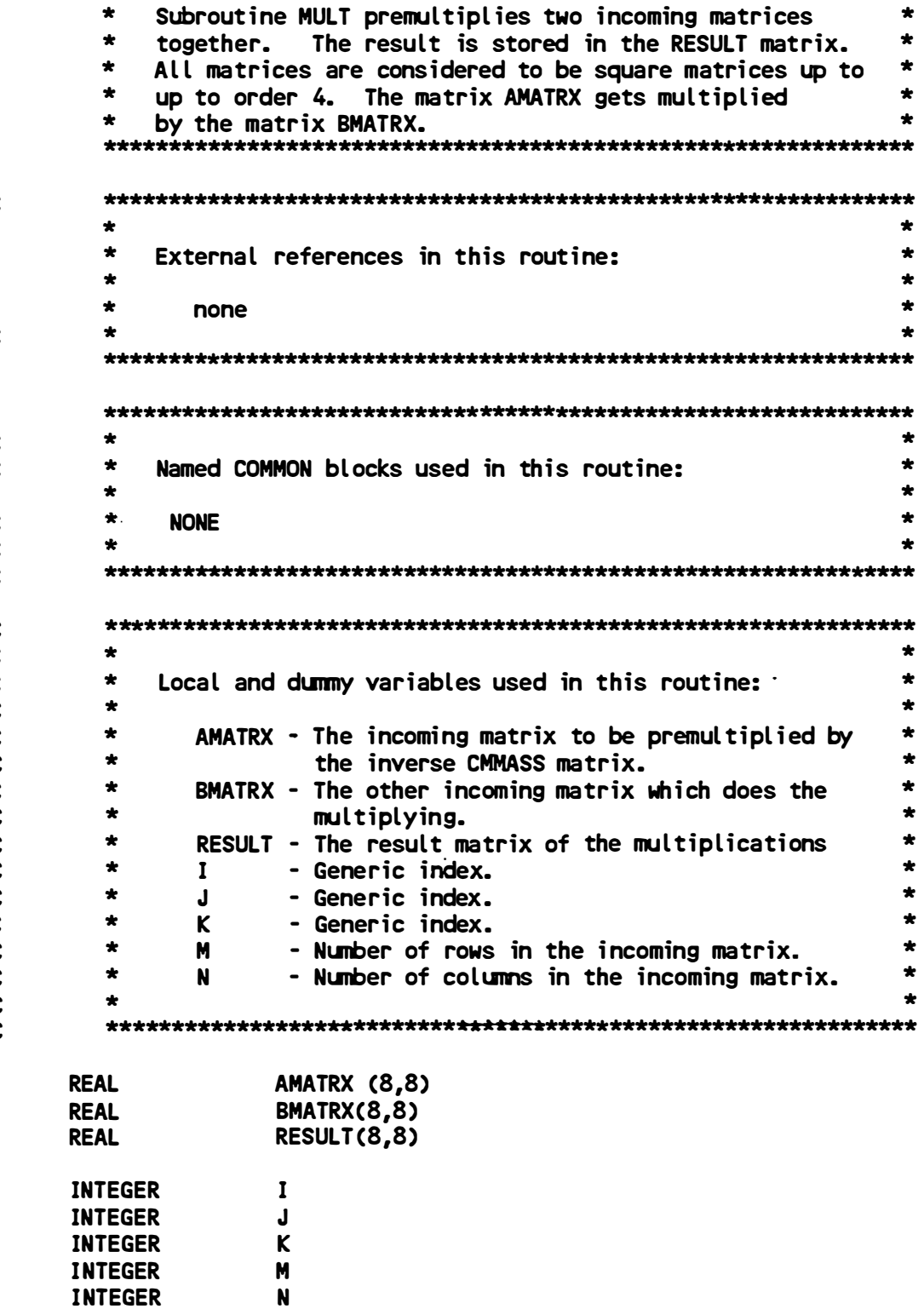

C

Multiply AMATRX by BMATRX putting the result into RESULT.

D.. $30 I=1, M$

DO $20 \mathrm{~J}=1, \mathrm{~N}$

$\operatorname{RESULT}(I, J)=0$.

DO $10 K=1, M$

$\operatorname{RESULT}(I, J)=\operatorname{RESULT}(I, J)+\operatorname{BMATRX}(I, K)$ * $\operatorname{AMATRX}(K, J)$

20

CONTINUE

30 CONTINUE

RETURN

END 


\section{Appendix D}

Module 2 Listing

D-1 


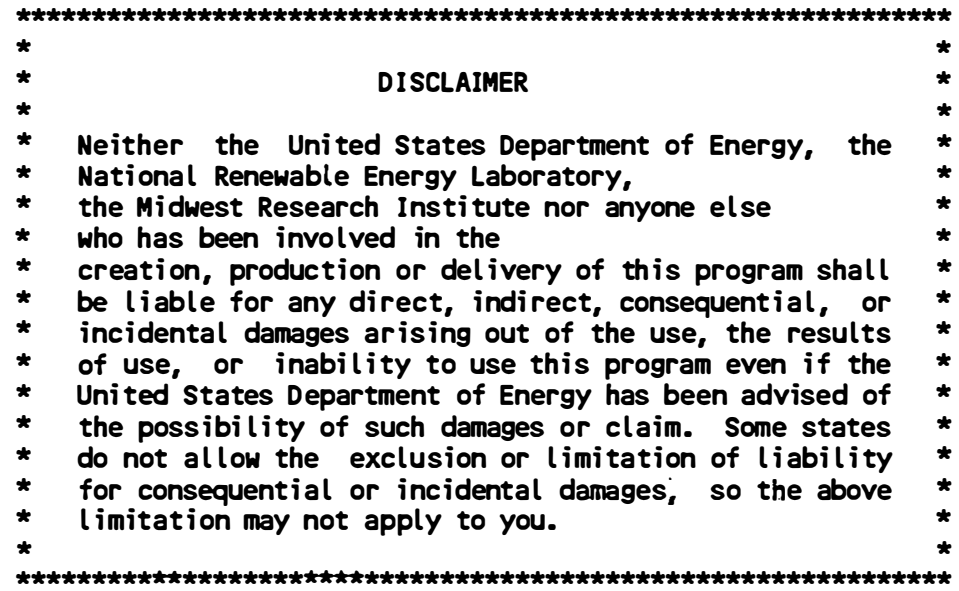

The STRAP Code
$\star$
$\star$
$\star$
$\star$




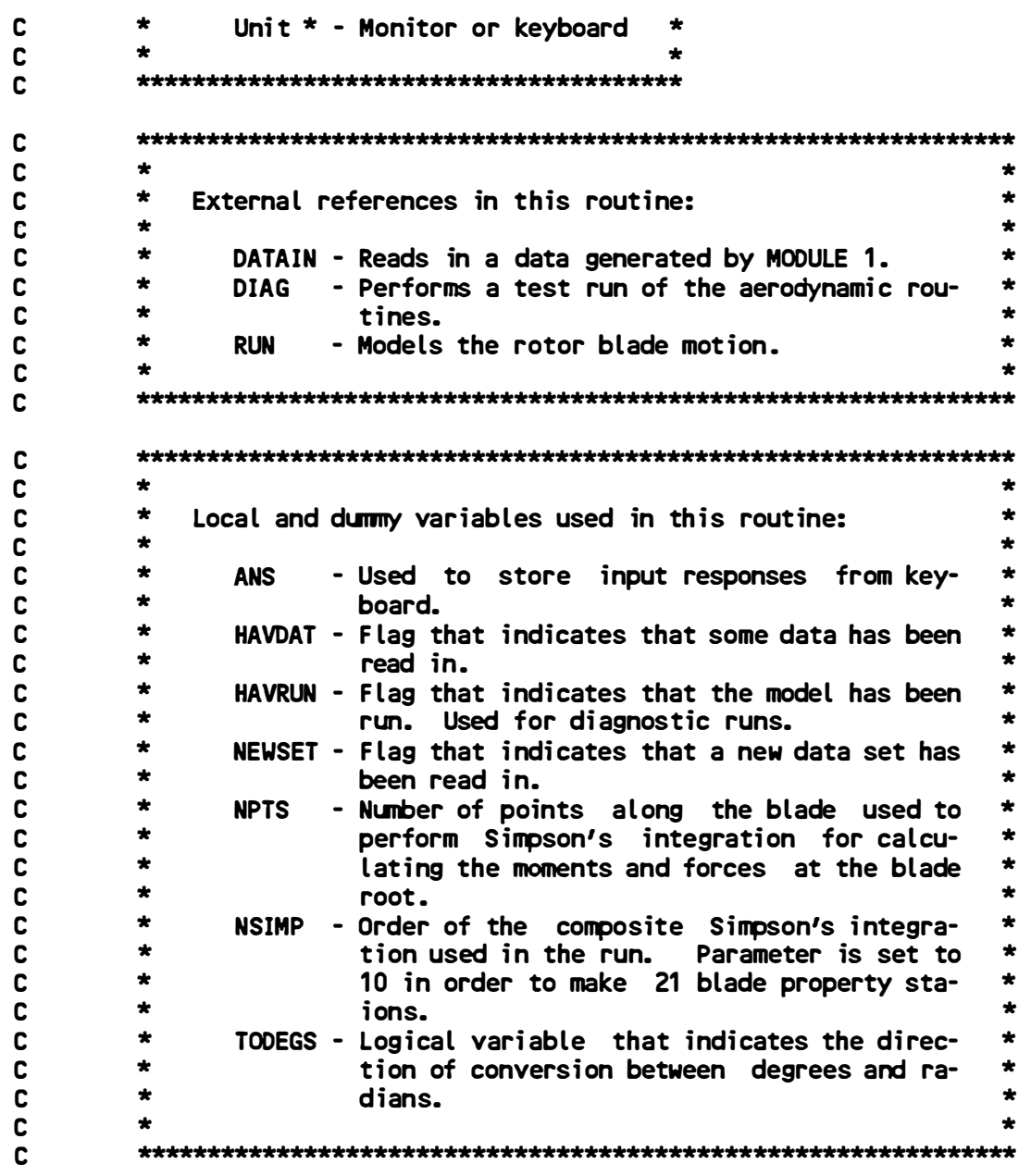

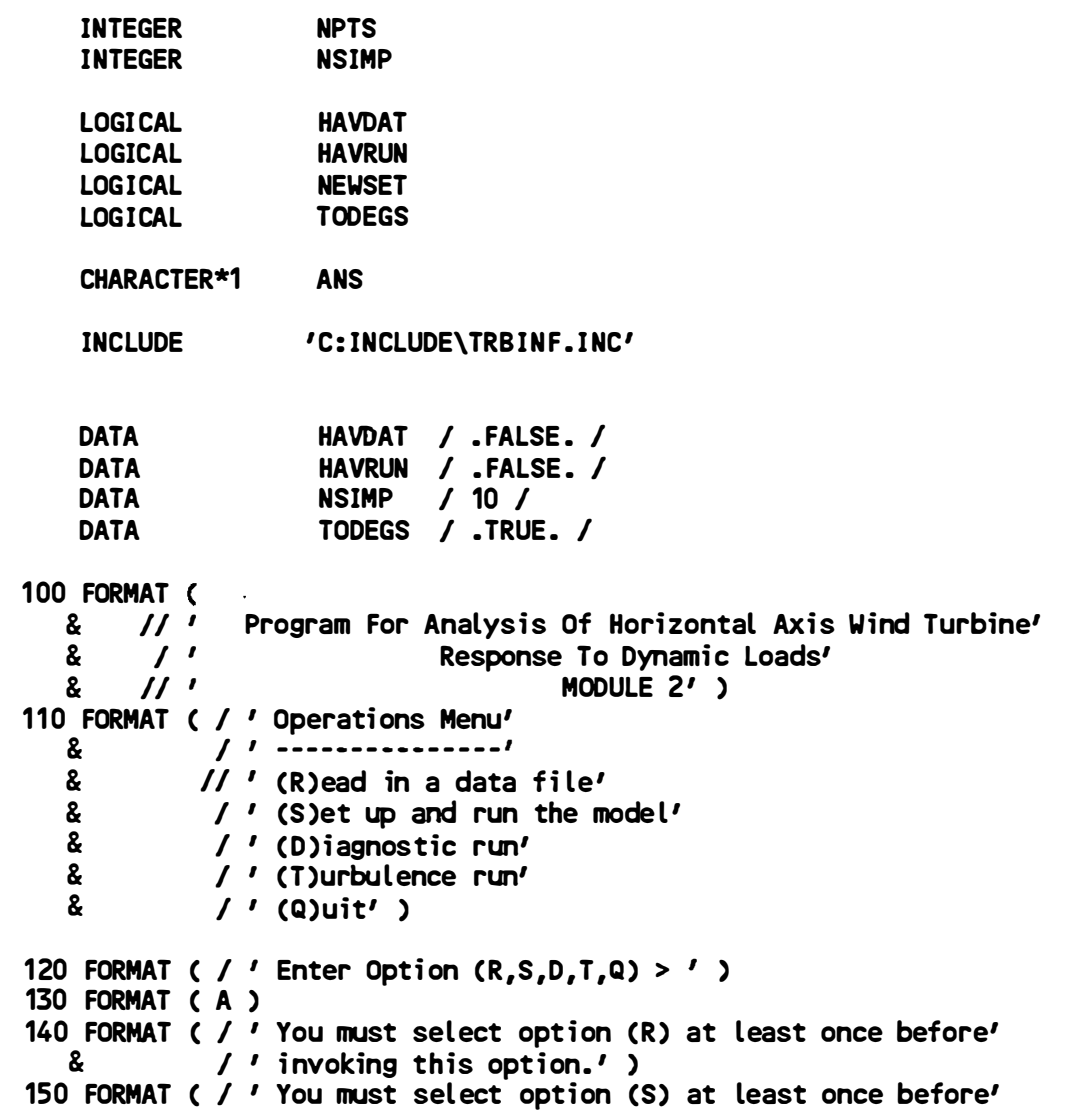


160 FORMAT ( / ' Invalid response. Please try again.' )

170 FORMAT ( / ' STRAP terminated normally.' / )

C Calculate the number of blade property stations.

NPTS $=2 *$ NSIMP +1

Print title.

PRINT 100

C

Print menu of options. Ask for choice.

10 PRINT 110

20 PRINT 120

READ 130, ANS

PRINT *, ANS

C

Which option was chosen?

IF ( ( ANS .EQ. 'R' ) .OR. ( ANS .EQ. ' $r$ ') ) THEN

C

Read in a data file. Convert limits values back to degrees if this isn't the first time we've read in data.

IF ( HAVDAT ) CALL CONVRT ( TODEGS )

CALL DATAIN

HAVDAT $=$. TRUE.

NEWSET $=$. TRUE.

GO TO 10

ELSE IF ( ( ANS .EQ. 'S') .OR. ( ANS .EQ. 'S') ) THEN

C

Set up and run the model. Option (R) must have been previously selected.

IF ( HAVDAT ) THEN CALL RUN ( NPTS, NEWSET , HAVRUN ) GO TO 10

ELSE

PRINT 140

GO TO 20

END IF

ELSE IF ( ( ANS .EQ. 'D' ) .OR. ( ANS .EQ. 'd' ) ) THEN

C

Run diagnostics. Option (S) must have been previously selected.

IF ( HAVRUN ) THEN CALL DIAG ( NPTS) GO TO 10

ELSE

PRINT 150

GO TO 20

END IF

ELSE IF ( ( ANS. EQ. ' $T$ ') ) .OR. ( ANS .EQ. ' $t$ ' ) ) THEN

C Run turbulence case. Option $S$ must have been previously invoked.

IF ( HAVRUN ) THEN

ITURB $=1$

CALL TRBCLC( NPTS)

GO TO 10

ELSE

PRINT 150

GO TO 20

ENDIF 
ELSE IF ( ( ANS .NE. ' $Q$ ' ) .AND. ( ANS .NE. ' $q$ ' ) ) THEN

C

Invalid response.

PRINT 160

GO TO 20

END IF

Processing complete.

PRINT 170

STOP

END

BLOCK DATA 
hub. (feet)

ALPHAO - The angle from the zero-lift line to the section-chord line. (Generally negative)

BEGIN2 - Azimuth position corresponding to the beginning of print region 2. (degrees)

BETAO - Blade coning angle. The value is input in degrees and converted to radians. (radians) (input data)

BLSHNK - Length of blade shank measured from the blade root to the start of the airfoil section. If airfoil begins at the root, then BLSHNK $=0$. (feet)

BLTIP - Blade length measured from the blade root to the blade tip. Note - rotor radius is HUBRAD + BLTIP. (feet)

CDZERO - Drag coefficient values at equidistant points along the blade. Derived from ACDZER values. (dimensionless)

CHI - Rotor pitch angle. Input in degrees and converted to radians. (radians) (input data)

CHORD - Blade chord at equidistant points along the blade. Derived from ACHORD. (feet)

CIFMOM - Integral of DIFMOM.

CKBEND - Bending stiffness matrix.

CKQLOD - Inertial moment stiffening matrix.

CKTCRL - Coriolis stiffening matrix.

CKTGRV - Gravity stiffening matrix.

CKTOMG - Centrifugal stiffening matrix.

CLALFA - Slope of the lift curve at equidistant points along the blade. Derived from ACLALF values. (radians ${ }^{\wedge}-1$ )

CLMAX - The maximm or stall value of the lift coefficient. Derived from ACLMAX values.

CMBLNC - Coefficient associated with the blade mass imbalance (OFFSET).

CMGRAV - Mass coefficients associated with gravitational loads.

CMGRV1 - Submatrix of CMGRAV containing odd terms only. Used only with teetering hubs.

CMMASS - Blade mass matrix. It is also-used in the inertia force stiffening term.

CMRGD1 - Submatrix of CMRIGD containing odd terms only. Used only with teetering hubs.

CMRIGD - Mass coefficients associated with rigid body motion.

CSUBMA - Pitching moment coefficient.

CTHP - Cosine of the pitch angle, THETAP.

DAETA - Differential aerodynamic forces on a blade section in the chordwise direction.

DAZETA - Differential aerodynamic forces on a blade section in the flapwise direction.

DELPSI - Delta-Psi. The amount Psi will change from this step to the next. Used in Euler predictor/corrector routine.

DELTAT - The incremental time change from one step to the next in the yaw solution (ITRIM=0).

DELTIM - Integral of DDELTI.

DELTVX - Turbulent wind velocity fluctuations. Not currently in use. (feet/second)

DELTVY - Turbulent wind velocity fluctuations. Not currently in use. (feet/second)

DELTVZ - Turbulent wind velocity fluctuations. Not currently in use. (feet/second)

DRGFRM - Drag coefficient form constant.

DVIND - The delta-V-induced velocity components. Components are of order epsilon.

ECNTFN - Complicated term.

EIAREA - Moment of inertia values at equidistant points along the blade. Derived from AEIARE data values. (Lb-Ft**2)

END2 - Azimuth position corresponding to the end of print region 2. (degrees)

ERROR - Difference between corrected and predicted value of the blade tip displacements in the Euler predictor/corrector routine.

ESUBAC - Distance from the blade elastic axis to the aerodynamic center. Positive towards the 
SO - Initial static blade deflection at the tip. It is set in subroutine STARTUP.

SHAPE - Array containing shape functions evaluated at regular intervals. The second subscript corresponds to Nth spatial derivative.

SHERXP - Wind shear power exponent. (input data)

SNEW - An array of tip displacement function values for each of the STEPMX stations around the disk. These are the results of the current computation. The zeroth element has the same value as the 360th element of SOLD.

SOLD - An array of tip displacement function values for each of the STEPMX stations around the disk. These are the results of the previous computation. The 360th element has the same value as the zeroth element of SNEW.

STEPMN - Minimm allowable azimuth angle step size. (degrees)

STEPMX - Maximm allowable azimuth angle step size. (degrees)

STHP - Sine of the pitch angle THETAP.

TCORLS - Blade tension coefficient due to coriolis * * effects.

TGRAV - Blade tension coefficient due to gravity effects.

THETAO - Orientation of the zero lift line with respect to the blade principal bending axis.

THETAP - Blade pitch angle. The angle from the flap bending axis (assumed to be the chord line) at the 3/4 spanwise location. The assumption has been made that the bending axis does not vary with spanwise location. In effect, THETAP sets the blade pitch angle and the direction of the flapping displacements. Input in degrees and converted to radians. (radians) (input data)

THETAT - The built-in blade twist angle from the section-chord line at the reference station to the section-chord line at the tip of the rotor. Positive towards feather.

TIME - Total elapsed time from the beginning of the yaw solution run. The zeroth element holds the past value of time, and the first element holds the current value. (seconds)

TITLE1 - First line of the data file title.

TITLE2 - Second line of the data file title.

TITLE3 - Third line of the data file title.

TOMGA - Blade tension coefficient associated with centrifugal force effects.

TRACEF - Flag that causes subroutine TRACE to print the values of various variables around the rotor disk.

TRMERR - Convergence criterion for computing the trim solution. (percent)

TSHADW The tower shadow velocity deficit.

TSUBO - Tower shadow wind speed offset component.

TSUBP - Tower shadow sinusoidal component.

VETA - Relative fluid velocity over the blade in the edgewise direction.

VHUB - Air speed at the height of the hub. (ft/sec) (input data)

VINDO - An induced velocity rotor term. It is uniform over the rotor disk.

VINDC - An induced velocity component associated with the Cosine(Psi) term.

VINDR - Inflow minus uniform induced velocity.

W - The actual tip displacements computed from the relation in subroutine FORM1: $V=$ Sum of $S(I) *$ GAMAA(I) and

VDOT = SUm of SDOT (I )*GAMMACI $)$, where $V$ is a function of position along the blade and time, $S$ is a function of time and GAMMA .is the blade coordinate shape function. The index I denotes summation over 


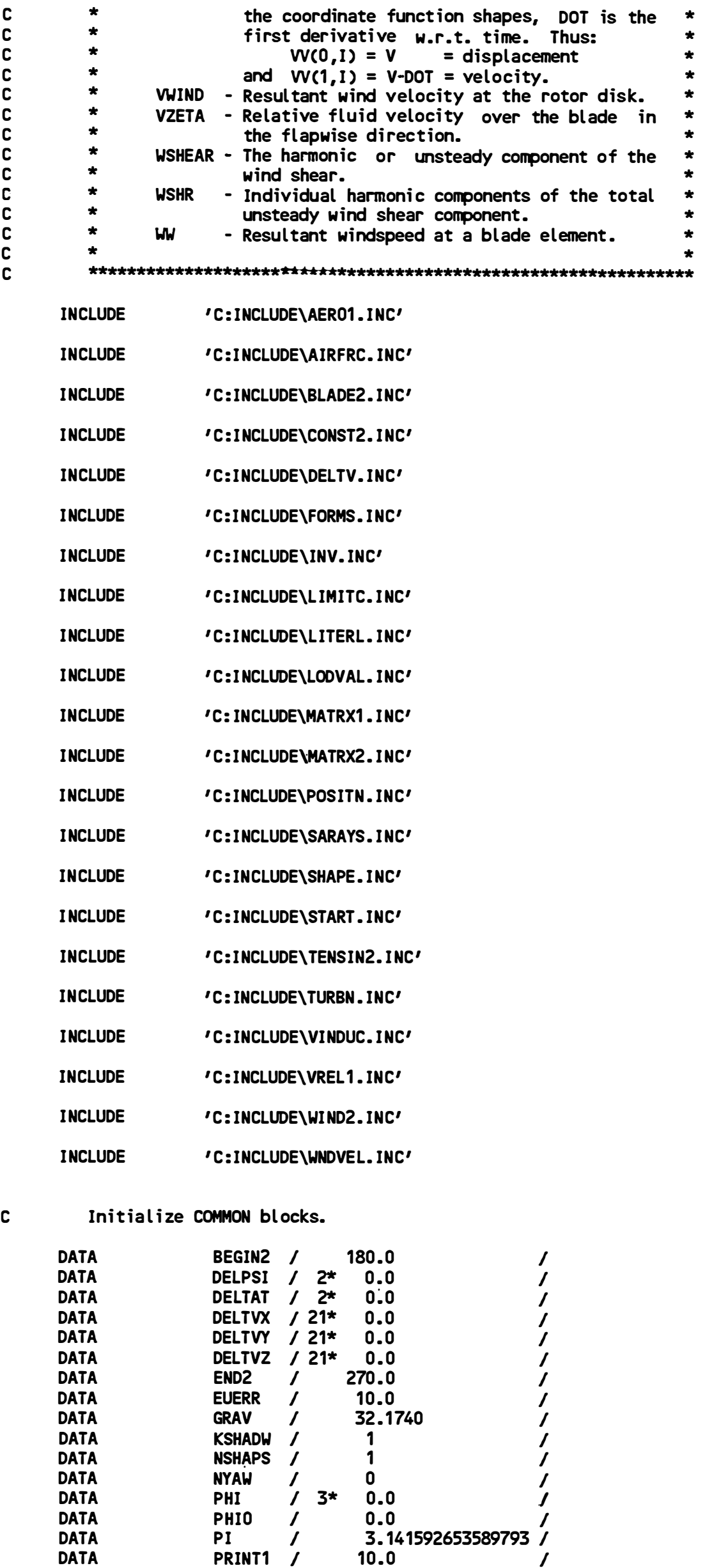




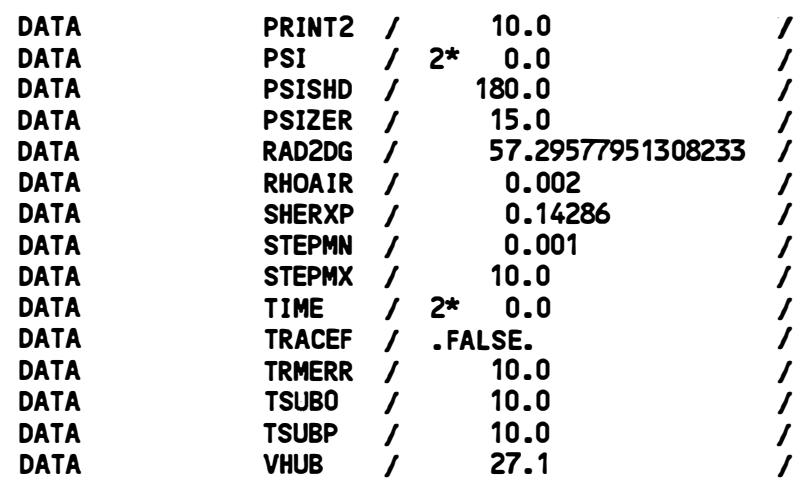

END

SUbROUtine ACCEL ( DEFLTN , VelCTY , ACCELN , NPTS , STEMP ) 
C2 - Omega*Phi*Cosine( Psi).

- dPhi/dt*sine( Psi ).

- -Chi*Cosine( ThetaP).

- Sine( ThetaP )*Sine( Psi ).

- Beta0*Cosine( ThetaP )*Cosine( Psi ).

- Omega^2.

- QUANT1^2.

CPSI - Cosine of the blade angle.

DD - Complicated term.

DEFLTN - The current value of the tip deflection, corresponding to the $S$ variable in the formulation of the equation of motion.

EE - QUANT1^2.

FAER01 - Aerodynamic force on blade \#1 of. teetering rotor.

FAER02 - Aerodynamic force on blade \#2 of teetering rotor.

FF - Omega*QUANT $1 *$ Cosine( ThetaP)

GG - Compl icated term.

$K \quad-$ Generic DO index.

$M \quad$ - Generic DO index.

N - Generic DO index.

NPTS - Number of points along the blade used to perform Simpson's integration for calculating the moments and forces at the blade root. (passed from STRAP1)

P1 - Array used in computing acceleration.

P2 - Array used in computing acceleration.

P3 - Array used in computing acceleration.

Q - Matrix used in computing acceleration.

QUANT1 - Omega*Sine( ThetaP ).

SPSI - Sine of the blade angle.

STEMP - Temporary array holding the tip displacement values. The third dimension represents the order of the time derivative.

VELCTY - The tip velocity corresponding to the S-dot value in the formulation of the equation of motion.

\begin{tabular}{|c|c|c|}
\hline $\begin{array}{l}\text { REAL } \\
\text { REAL } \\
\text { REAL } \\
\text { REAL } \\
\text { REAL } \\
\text { REAL } \\
\text { REAL } \\
\text { REAL } \\
\text { REAL } \\
\text { REAL } \\
\text { REAL } \\
\text { REAL } \\
\text { REAL } \\
\text { REAL } \\
\text { REAL } \\
\text { REAL } \\
\text { REAL } \\
\text { REAL } \\
\text { REAL } \\
\text { REAL } \\
\text { REAL } \\
\text { REAL } \\
\text { REAL } \\
\text { REAL } \\
\text { REAL } \\
\text { REAL } \\
\text { REAL }\end{array}$ & $\begin{array}{l}\text { AA } \\
\text { ACCELN } \\
\text { BB } \\
\text { BLDANG } \\
\text { C1 } \\
\text { C2 } \\
\text { C3 } \\
\text { C4 } \\
\text { C5 } \\
\text { C6 } \\
\text { C7 } \\
\text { CC } \\
\text { CPSI } \\
\text { DEFLTN } \\
\text { EE } \\
\text { FAER01 } \\
\text { FAER02 } \\
\text { FF } \\
\text { P1 } \\
\text { P2 } \\
\text { P3 } \\
\text { Q } \\
\text { QUANT1 } \\
\text { SPSI } \\
\text { STEMP } \\
\text { VELCTY } \\
\text { THETAC }\end{array}$ & $\begin{array}{l}(4) \\
(4) \\
(4) \\
(4) \\
(4) \\
(4,4) \\
(4,0: 1,0: 2) \\
(4)\end{array}$ \\
\hline $\begin{array}{l}\text { INTEGER } \\
\text { INTEGER } \\
\text { INTEGER } \\
\text { INTEGER }\end{array}$ & $\begin{array}{l}\dot{K} \\
\mathbf{M} \\
\mathbf{N} \\
\text { NPTS }\end{array}$ & \\
\hline INCLUDE & 'C:INCLI & UDE \AIRFRC.INC \\
\hline INCLUDE & 'C:INCLI & UDE \BLADE2.INC \\
\hline
\end{tabular}




\begin{tabular}{|c|c|}
\hline INCLUDE & 'C:INCLUDE ICONST2.INC' \\
\hline INCLUDE & 'C:INCLUDE MATRX1.INC' \\
\hline INCLUDE & 'C: INCLUDEVMATRX2. INC' \\
\hline INCLUDE & 'C:INCLUDEXPOSITN.INC' \\
\hline INCLUDE & 'C: INCLUDE ITURBN. INC' \\
\hline INCLUDE & 'C: INCLUDEXTENSIN2.INC' \\
\hline INCLUDE & 'C:INCLUDEXFORMS. INC' \\
\hline INCLUDE & 'C:INCLUDE \HUBPRP2.INC' \\
\hline INCLUDE & 'C:INCLUDEISPRING2. INC' \\
\hline
\end{tabular}

C Calculate the aero forces. The aero subroutines need to be C called twice, once for each blade. The first call is for

C blade \#2 at a blade angle of Psi+180 degrees and the second C call is for blade \#1 at a blade angle of Psi.

BLDANG $=\operatorname{PSI}(1)+P I$

Calculate aerodynamic loads on blade \#2.

CALL FORM1 ( STEMP , NPTS , NSHAPS , BLDANG )

THETAC $=$ THETAP + BETA*SDELT3

STHC = SIN(THETAC)

CTHC $=$ COS (THETAC)

TTHC = TAN(THETAC)

CALL AFORCE ( NPTS, BLDANG )

DO $70 M=1$, NSHAPS

$70 \quad$ FAERO2 (M) = FAERO(M)

PRINT *, 'FAERO2 = ', FAER02(1)

BLDANG = PSI (1)

C

Calculate aerodynamic loads on blade \#1.

CALL FORM1 ( STEMP， NPTS , NSHAPS , BLDANG )

THETAC $=$ THETAP + BETA*SDELT3

STHC = SIN(THETAC)

CTHC $=$ COS(THETAC)

TTHC = TAN(THETAC)

CALL AFORCE ( NPTS, BLDANG )

C

C

C

Sum the aerodynamic loads for both blades. For the asymetric modes $(M=1,3)$, the difference in the aerodynamic loads is actually found.

DO $80 \mathrm{M}=1$, NSHAPS

FAERO1 (M) $=$ FAERO(M)

FAERO $(M)=0.5 *(\operatorname{FAER01}(M)+\operatorname{FAERO2}(M) *(-1) * * M)$

80

CONTINUE

C

Calculate constant coefficients.

$$
\begin{aligned}
& \text { CTHP = COS(THETAP) } \\
& \text { STHP = SIN(THETAP) } \\
& \text { SPSI = SIN( BLDANG) } \\
& \text { CPSI = COS( BLDANG) } \\
& \text { QUANT1 = OMEGA*STHP }
\end{aligned}
$$




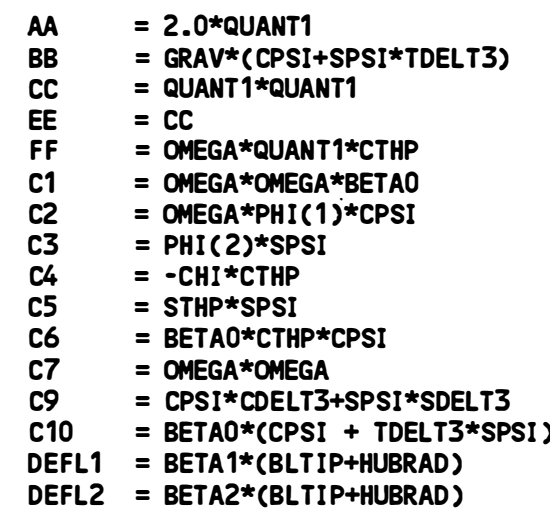

C Compute P1 (see formulation notes).

DO 85 M=2, NSHAPS

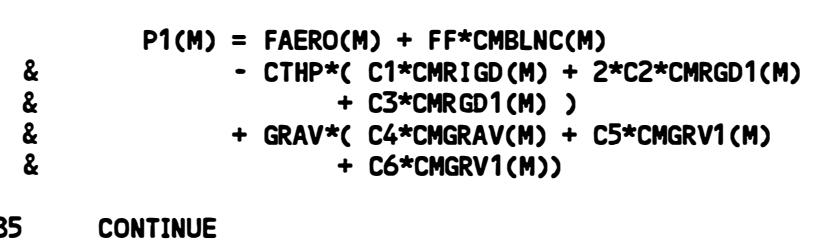

$\&$

$P 1(1)=$ FAERO $(1) * C T H P-(2 . * C 2+C 3) * C M R G D 1(1)$

+ GRAV*C10*CMGRV1(1) - C9*GRAV*CMHUB1 (1)

C Compute P2 - $S(M)$ product (see formulation notes).

DO $20 M=2$, NSHAPS

$P 2(M)=E E * D E F L T N(M)$

20 CONTINUE

IF( DEFLTN(1) .LE. 0.) THEN

IF(ABS(DEFLTN(1)) .LT. DEFL1) THEN P2(1) = -1.*KOSTIF(1)*DEFLTN(1)

ELSEIF(ABS (DEFLTN(1)) .LT. DEFL2) THEN

\& $P 2(1)=-1 . *(K O S T I F(1)+K 1 S T I F(1)) * \operatorname{DEFLTN}(1)$

ELSE

\& $\quad-K 2 S T I F(1) * D E F L 2-1 . \star D A M P(1) * V E L C T Y(1)$

ENDIF

ELSE

IF(DEFLTN(1) .LT. DEFL 1) THEN

P2(1) = -1.*KOSTIF (1)*DEFLTN(1)

ELSEIF(DEFLTN(1) .LT. DEFL2) THEN

$\&$

P2(1) $=-1 . *(K O S T I F(1)+K 1 S T I F(1)) * \operatorname{DEFLTN}(1)$ $+K 1 S T$ IF (1)*DEFL1 - 1.*DAMP(1)*VELCTY (1)

ELSE

$\&$

$P 2(1)=-1 . *(K O S T I F(1)+K 2 \operatorname{STIF}(1)) * \operatorname{DEFLTN}(1)$ $+K 2 \operatorname{STIF}(1) * D E F L$.2 - 1.*DAMP (1)*VELCTY(1)

END
ENDIF

C Compute $Q(M, K)$ - $S(K)$ products (see formulation notes).

DO $50 M=1$,NSHAPS

$P 3(M)=0.0$

DO $40 \mathrm{~K}=1$, NSHAPS 
DO $30 \mathrm{~N}=1$, NSHAPS

$$
P 3(M)=P 3(M)+Q(M, K) * D E F L T N(K)
$$

\section{CONTINUE}

DO $60 M=1$, NSHAPS

60 ACCELN(M) $=P 1(M)+P 2(M)+P 3(M)$

RETURN

END

SUBROUTINE AERO ( NPTS , BLDANG ) 


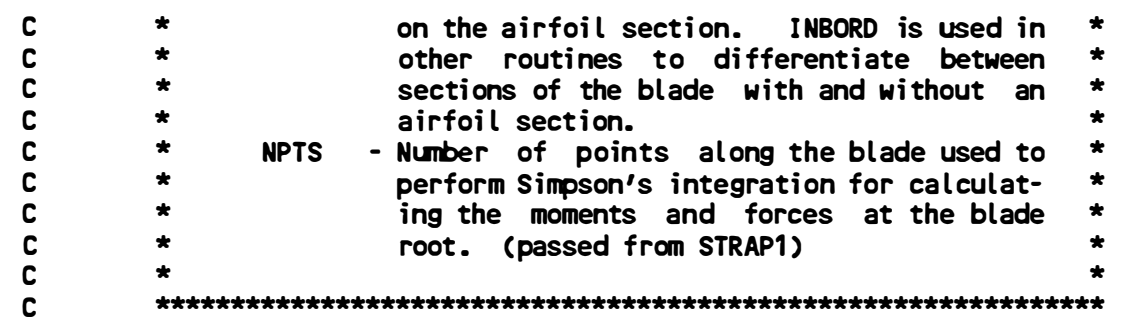

\begin{tabular}{|c|c|}
\hline $\begin{array}{l}\text { REAL } \\
\text { REAL } \\
\text { REAL } \\
\text { REAL } \\
\text { REAL }\end{array}$ & $\begin{array}{l}\text { ALPHA } \\
\text { BLDANG } \\
\text { CD2 } \\
\text { CDRAG } \\
\text { CLIFT }\end{array}$ \\
\hline $\begin{array}{l}\text { INTEGER } \\
\text { INTEGER } \\
\text { INTEGER }\end{array}$ & $\begin{array}{l}\text { I } \\
\text { INBORD } \\
\text { NPTS }\end{array}$ \\
\hline INCLUDE & 'C:INCLUDE\AER01.INC' \\
\hline INCLUDE & 'C:INCLUDE IAIRFRC.INC' \\
\hline INCLUDE & 'C: INCLUDE \BLADE2. INC' \\
\hline INCLUDE & 'C:INCLUDEXCONST2.INC' \\
\hline INCLUDE & 'C:INCLUDE TTURBN. INC' \\
\hline INCLUDE & 'C: INCLUDEXPOSITN. INC' \\
\hline INCLUDE & 'C:INCLUDEIVREL1.INC' \\
\hline
\end{tabular}

CALL VREL ( INBORD， NPTS , BLDANG ) of the blade - that is, the portion of the blade without any airfoil section. This is related to BLSHNK.

DO $10 \mathrm{I}=1$, NPTS

IF ( I . LT. INBORD ) THEN

$$
\begin{array}{ll}
\operatorname{DAETA}(I) & =0.0 \\
\operatorname{DAZETA}(I) & =0.0 \\
W W(I) & =0.0
\end{array}
$$

ELSE

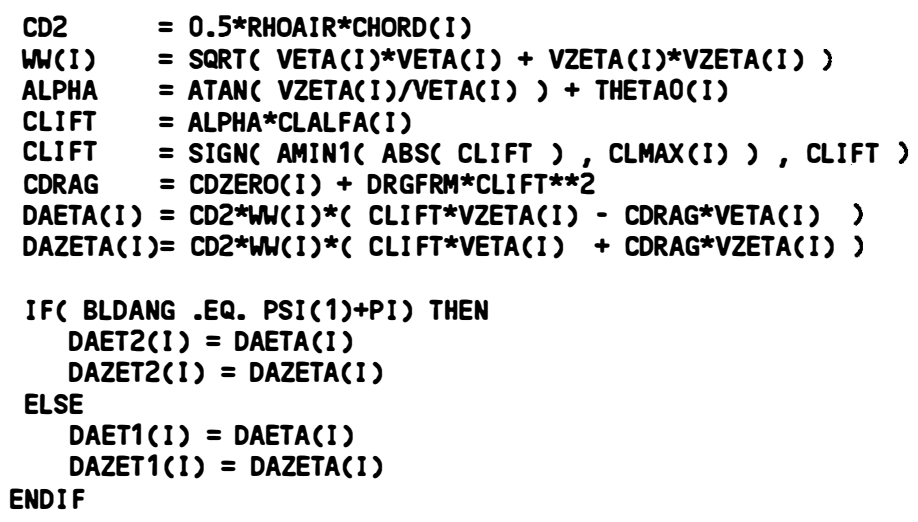


END IF

\section{RETURN}

END

SUBROUTINE AFORCE ( NPTS , BLDANG )

External references in this routine:

AERO - Calculates the aerodynamic forces on the blade.

SIMPSN - Composite Simpson's integration.

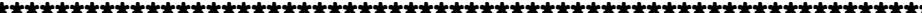

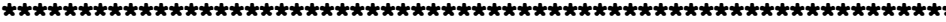

Named COMMON blocks used in this routine:

AIRFRC Holds values used in aerodynamic calculations.

BLADE - Holds blade property values such as stiffness and mass distributions.

MATRX1 - Holds stiffness coefficient matrices.

TURBN - Holds turbine parameters such as number of blades, rotor speed, etc.

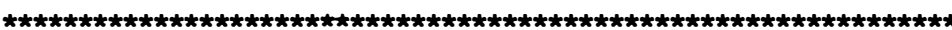

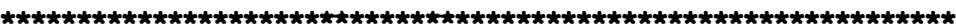

Local and dumy variables used in this routine:

BLDANG - Blade azimuth position used in the teetering rotor option.

FOFZET - Product of the dA(Zeta) and the shape function.

FTEMP - Temporary array.

I - Generic index.

L - Generic index.

M - Generic index.

NPTS - Number of points along the blade used to perform Simpson's integration for calculating the moments and forces at the blade root. (passed from STRAP1) $*$ 


\begin{tabular}{|c|c|}
\hline INCLUDE & 'C:INCLUDE\AIRFRC.INC' \\
\hline INCLUDE & 'C:INCLUDE\BLADE2. INC' \\
\hline INCLUDE & 'C:INCLUDE MMATRX1.INC' \\
\hline INCLUDE & 'C:INCLUDE\SHAPE.INC' \\
\hline INCLUDE & 'C:INCLUDE \TURBN. INC' \\
\hline
\end{tabular}

Compute aerodynamic forces.

CALL AERO ( NPTS, BLDANG)

Compute the product of the DAZETA value and the shape
C function at each blade station for each specified shape
C function.

DO $20 \mathrm{M}=1$, NSHAPS

DO $10 \mathrm{I}=1$, NPTS

10 FOFZET (I) $=$ DAZETA $(I) * \operatorname{SHAPE}(M, 0, I)$

C Compute the integral of $\mathrm{dF}(A e r 0)$ along the blade.

FAERO(M) $=$ SIMPSN( 0.0, BLTIP , NPTS , FOFZET )

20 CONTINUE

C Multiply the F-AERO integrals by the inverse of the CMMASS C coefficient matrix. This is done for the specified number C of shape functions.

DO 50 M=1, NSHAPS

$\operatorname{FTEMP}(M)=0.0$

DO 40 L=1, NSHAPS

$40 \quad \operatorname{FTEMP}(M)=\operatorname{FTEMP}(M)+\operatorname{CMMASS}(M, L) * F A E R O(L)$

50 CONTINUE

C Replace the F-AERO values by their corresponding values

C multiplied by the CMMASS inverse coefficient matrix.

DO $60 \mathrm{M}=1$, NSHAPS

60 FAERO(M) $=$ FTEMP $(M)$

RETURN

END

SUBROUTINE CAPS ( STRING )

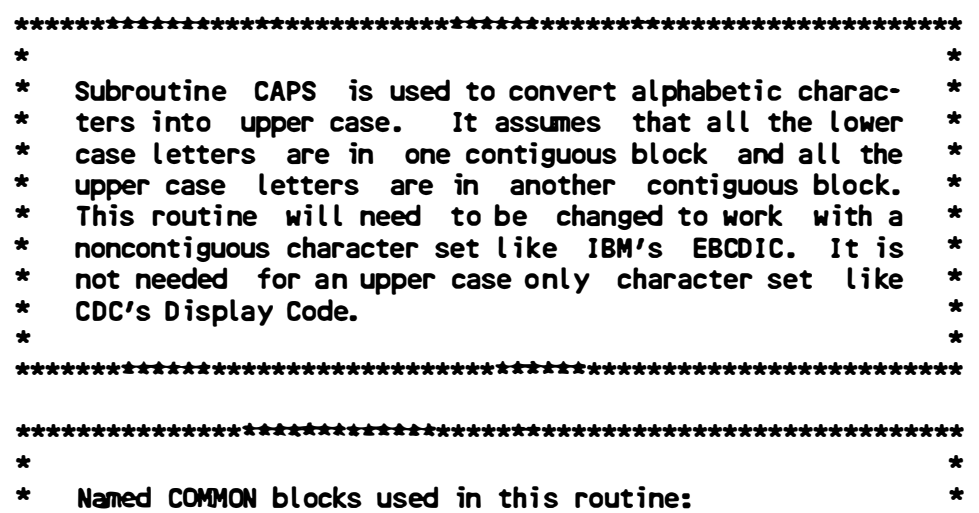


none

(1)

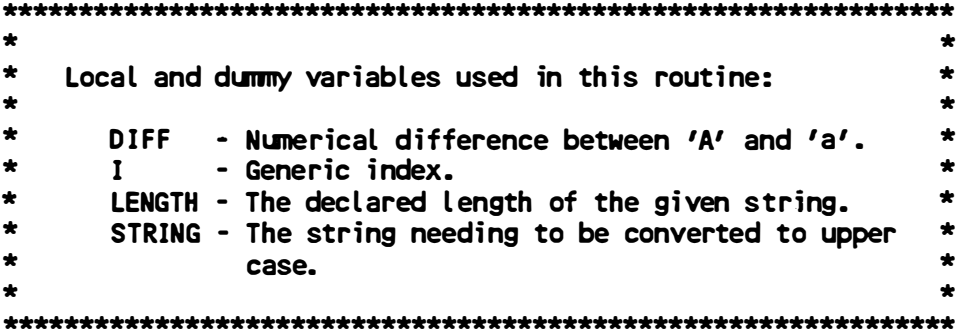

INTEGER DIFF

INTEGER I

INTEGER LENGTH

CHARACTER*(*) STRING

DIFF = ICHAR( 'a' ) - ICHAR( 'A' )

Get the length of the string.

LENGTH = LEN( STRING )

Look for lower case letters. Convert them to upper, case.

DO $100 \mathrm{I}=1$, LENGTH

$\&$

IF ( ( STRING(I:I) .GE. 'a' ) .AND. ( STRING(I:I) .LE. ' $Z$ ') ) THEN

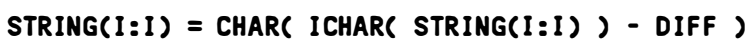

END IF

100 CONTINUE

RETURN

END

SUBROUTINE CONVRT ( TODEGS )

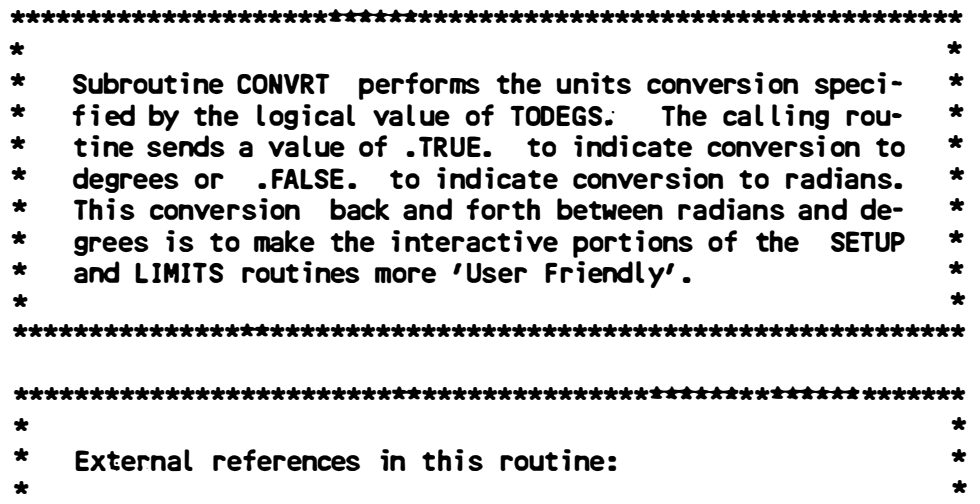




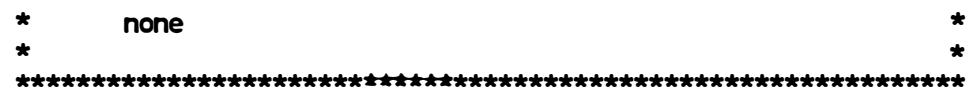

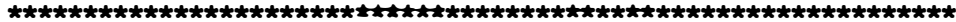
$\star$

Named COMMON blocks used in this routine:

CONST Turbine and other constants used in load calculations.

LIMITC - Holds values used in the LIMITS routine.

blades, rotor speed, etc.

WIND - Holds wind shear and tower shadow parame- * ters.

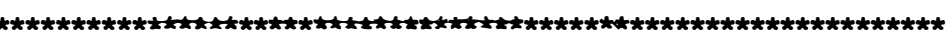

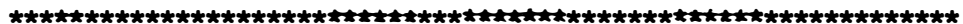
$*$

* Local and dumiry variables used in this routine:

* FACT1 - Conversion factor.

FACT2 - Conversion factor.

TODEGS - Logical variable that indicates the direction of conversion between degrees and radians.

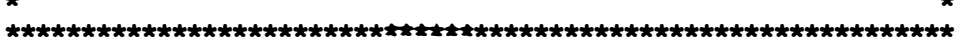

$\begin{array}{ll}\text { REAL } & \text { FACT1 } \\ \text { REAL } & \text { FACT2 }\end{array}$

LOGICAL TODEGS

INCLUDE 'C:INCLUDEICONST2.INC'

INCLUDE 'C:INCLUDEILIMITC.INC'

INCLUDE 'C:INCLUDEITURBN.INC'

INCLUDE 'C:INCLUDEIWIND2.INC'

INCLUDE 'C:INCLUDEISPRINGZ2.INC'

C For all calls to this routine, set the conversion factors C specific to the unit conversion required. Then perform the C conversions and return to calling routine.

IF ( TODEGS ) THEN

C

Convert from radians and radians/second to degrees and RPM.

FACT1 $=$ RAD2DG

FACT2 $=30.0 / P I$

ELSE

Convert from degrees and RPM to radians and radians/second.

FACT1 $=1.0 /$ RAD2DG

FACT2 $=\mathrm{PI} / 30.0$

END IF

C

Convert 'free' variables from the SETUP routine.

$$
\begin{aligned}
& \text { BETAO = FACT 1*BETAO } \\
& \text { ALPHAO }=\text { FACT } 1 * A L P H A O \\
& \mathrm{CHI}=\text { FACT } 1 * \text { CHI } \\
& \text { PHIAMP. = FACT } 1 * \text { PHIAMP } \\
& \text { PHIOMG = FACT } 1 * \text { PHIOMG } \\
& \text { PHIO = FACT1*PHIO } \\
& \text { PSISHD }=\text { FACT } 1 * P S I S H D
\end{aligned}
$$


THETAT = FACT $1 *$ THETAT

DELT3 = FACT1*DELT3

BETA1 = FACT1*BETA1

BETA2 = FACT1*BETA2

OMEGA = FACT2*OMEGA

BEGIN2 = FACT1*BEGIN2
END2 = FACT1*END2
PRINT1 = FACT1*PRINT1
PRINT2 = FACT1*PRINT2
STEPMX = FACT1*STEPMX
STEPMN = FACT1*STEPMN

RETURN

END

SUBROUT INE DATAIN 


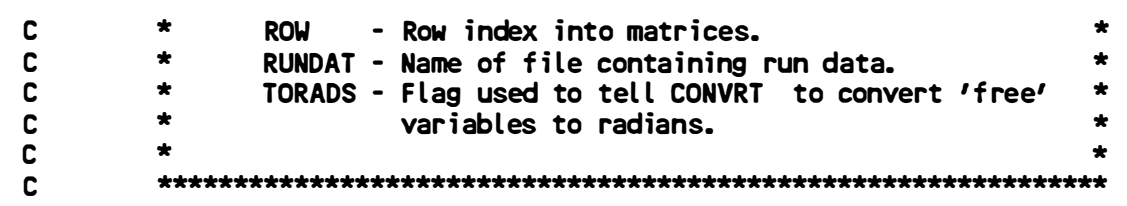

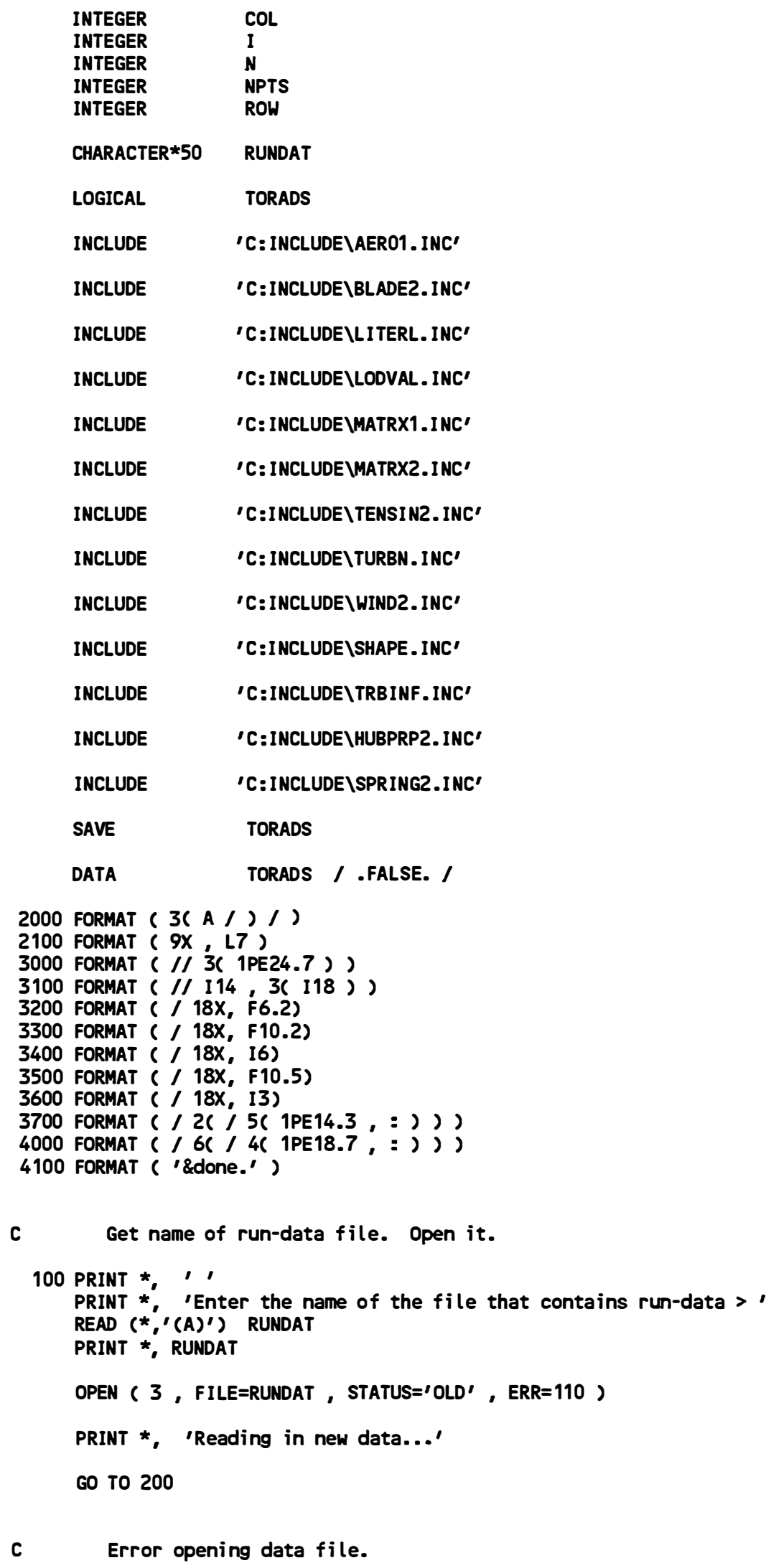


110 PRINT *, , $\gg$ Run-data file not found. Try again. $\ll<'$ GO TO 100

C Read titles and hub type.

200 READ (3,2000) TITLE1 , TITLE2, TITLE3

C

Read scalar data.

$\begin{array}{ll}\text { READ }(3,3000) & \text { ALENTH , ALPHAO , BETAO } \\ \text { READ }(3,3000) & \text { BLSHNK , BLTIP , CHI } \\ \text { READ }(3,3000) & \text { CSUBMA , DRGFRM , HUBHT } \\ \text { READ }(3,3000) & \text { HUBRAD , OMEGA , PHIO } \\ \text { READ }(3,3000) & \text { PHIAMP , PHIOMG , PSIZER } \\ \text { READ }(3,3000) & \text { SHERXP , THETAP , THETAT } \\ \text { READ }(3,3000) & \text { TSUBO , TSUBP , VHUB } \\ \text { READ }(3,3100) & \text { KSHADW , NBLADS , NSHAPS, NPTS } \\ \text { READ }(3,3200) & \text { DELT3 } \\ \text { READ }(3,3200) & \text { UNDSLG } \\ \text { READ }(3,3200) & \text { HUBMAS } \\ \text { READ }(3,3200) & \text { HUBDIS } \\ \text { READ }(3,3200) & \text { BETA1 } \\ \text { READ }(3,3200) & \text { BETA2 } \\ \text { READ }(3,3400) & \text { NUMSCN } \\ \text { READ }(3,3500) & \text { TIMINC } \\ \text { READ }(3,3600) & \text { MSTAT } \\ \text { READ }(3,3700) & \text { ( STA(I), I=1,MSTAT) }\end{array}$

C

Read in vector values.

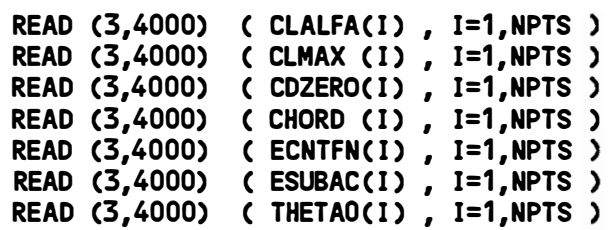

C Read in coefficient matrices.

READ (3,4000) ( ( CKBEND (ROW,COL), COL=1,4), ROW=1,4)

READ $(3,4000)$ ( ( CKTOMG(ROW,COL), COL=1,4), ROW=1,4)

READ $(3,4000)$ ( ( CKTGRV(ROW, COL), COL=1,4), ROW=1,4)

$\operatorname{READ}(3,4000)$ ( ( CKQLOD (ROW, COL), COL $=1,4), \operatorname{ROW}=1,4$ )

$\operatorname{READ}(3,4000)$ ( ( CMMASS(ROW,COL), COL=1,4), ROW=1,4)

DO $300 \mathrm{~N}=1,4$

$300 \operatorname{READ}(3,4000)$ ( ( CKTCRL $(N, R O W, C O L)$, COL=1,4 ), ROW=1,4 )

C Read in vector coefficients.

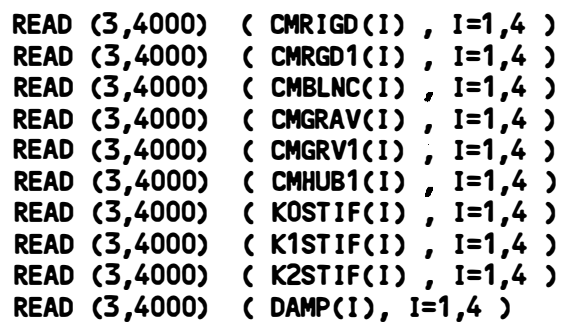

C

Read tension arrays.

DO $400 \mathrm{~N}=1,4$

$400 \operatorname{READ}(3,4000) \quad(\operatorname{TCORLS}(N, I), I=1, N P T S)$

READ $(3,4000)$ ( TGRAV(I) , I=1, NPTS )

$\operatorname{READ}(3,4000)$ ( TOMGA(I) , I=1,NPTS )

DO $410 \mathrm{~N}=1,4$

$410 \operatorname{READ}(3,4000)(\operatorname{CIFMOM}(N, I), I=1, N P T S)$ 
READ $(3,4000)$ ( DELTIM(I) I=1,NPTS )

READ $(3,4000)$ ( OFFMAS(I), I=1, NPTS)

C Read in modeshapes and derivatives calculated in
C module 1. Modes 1 and 3 are the rigid teeter and
C first asymetric bending, while modes 2 and 4
C
are first and second symetric bending. For frequency
information see the modeshape file produced as a result
o
of running module 1.
C
C There is now no longer any need for the old SUBROUTINE
C
SHAPES as the modeshapes are read in directly from this
input file into the shape arrays, instead of re-calculating
them as done in the "old" FLAP version.

DO $520 \mathrm{I}=1$,NPTS

$\operatorname{READ}(3, *)(\operatorname{SHAPE}(J, 0,1), J=1,4)$

520 CONTINUE

DO $530 \mathrm{I}=1$, NPTS

$\operatorname{READ}(3, *)(\operatorname{SHAPE}(J, 1,1), J=1,4)$

530 CONTINUE

DO $540 I=1$,NPTS

540 CONTINUE

$\operatorname{READ}(3, *)(\operatorname{SHAPE}(J, 2,1), J=1,4)$

C

Processing complete. Close data file.

PRINT 4100

CLOSE ( 3 )

PRINT *, 'Analyzing a teetering hub.'

The units are converted to radians and radians/second to permit compatibility between newly input data and data being used for consecutive model runs. Thus the DIAG and RUN routines do not need to know if the data they are using is new or old.

CALL CONVRT ( TORADS )

CDELT3 $=$ COS(DELT3)

SDELT3 = SIN(DELT3)

TDELT3 = TAN(DELT3)

CDAMP $=$ DAMP(1)*BLTIP*BLTIP

RETURN

END

SUBROUTINE DIAG ( NPTS ) 


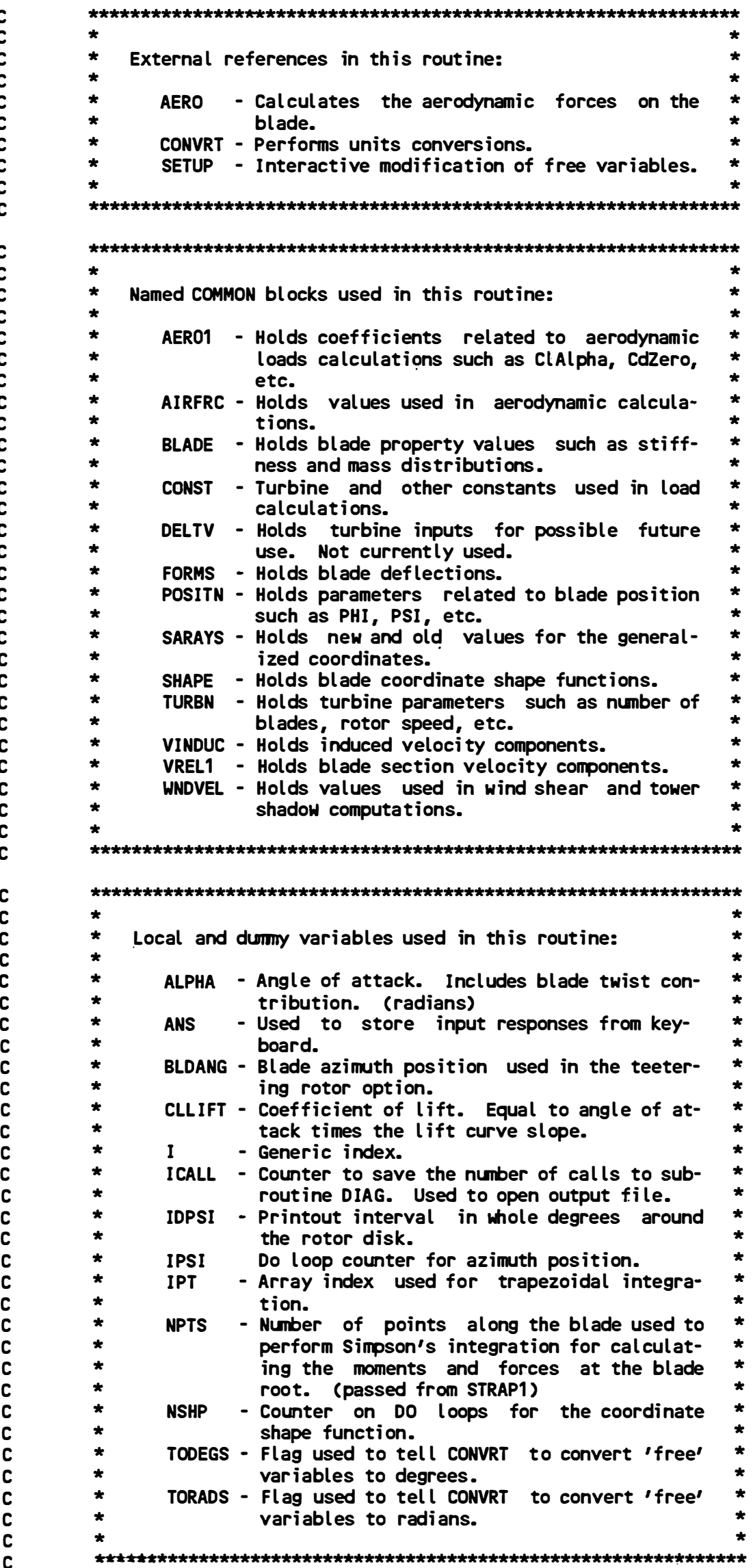

REAL ALPHA (21) 


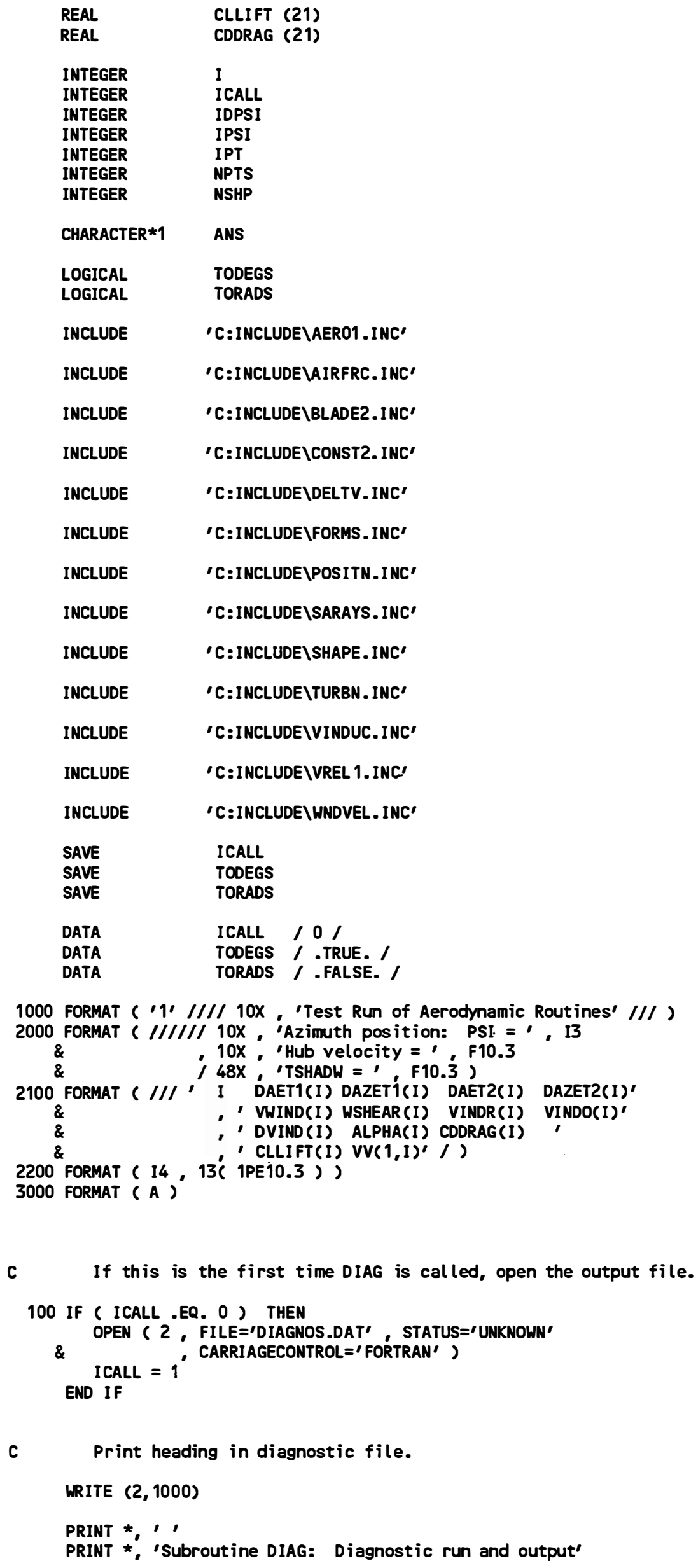


PRINT *, , '

Convert values into degrees.

CALL CONVRT ( TODEGS )

C

Run setup routine.

CALL SETUP

C

Convert values back into radians for internal use.

CALL CONVRT ( TORADS )

C

Ask for printout interval.

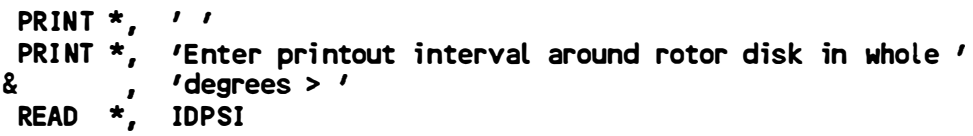

C

NOTE: DIAG assumes that time is frozen at time $=0$.

PHI $(0)=$ PHIO

PHI (1) = PHIOMG

PHI (2) $=0.0$

C

Go around the disk in IDPSI increments.

DO 250 IPSI $=0,360$, IDPSI

C

Clear the forms array.

PSI (1) $=$ IPSI $/$ RAO2DG

BLDANG = PSI $(1)$

Set up the forms array.

203

DO $200 \mathrm{I}=1$, NPTS

$W(0, I)=0.0$

$W(1,1)=0.0$

$V 1(0,1)=0.0$

200

$v_{1}(1,1)=0.0$

CONTINUE

DO 220 IPT $=1$, NPTS

IF( BLDANG .EQ. PSI(1)+PI) THEN

$V 1(0, I P T)=V 1(0, I P T)-\operatorname{SHAPE}(1,0, I P T) \star \operatorname{SNEW}(1,0$, IPSI $)$

$V 1(1, I P T)=V 1(1, I P T)-\operatorname{SHAPE}(1,0$, IPT $) * \operatorname{SNEW}(1,1$, IPSI $)$

ELSE

$\operatorname{V1}(0, I P T)=V 1(0, I P T)+\operatorname{SHAPE}(1,0, I P T) * \operatorname{SNEW}(1,0$, IPSI $)$

$V 1(1, I P T)=V 1(1, I P T)+\operatorname{SHAPE}(1,0, I P T) \star \operatorname{SNEW}(1,1$, IPSI $)$

ENDIF

DO 210 NSHP $=1$, NSHAPS

IF( BLDANG .EQ. PSI(1)+PI) THEN

MARK $=(-1) *$.$* NSHP$

ELSE

MARK $=1$

ENDIF

$W(0, I P T)=W(0, I P T)$

+ MARK^SHAPE(NSHP, O, IPT )*SNEW(NSHP, 0, IPSI ) 


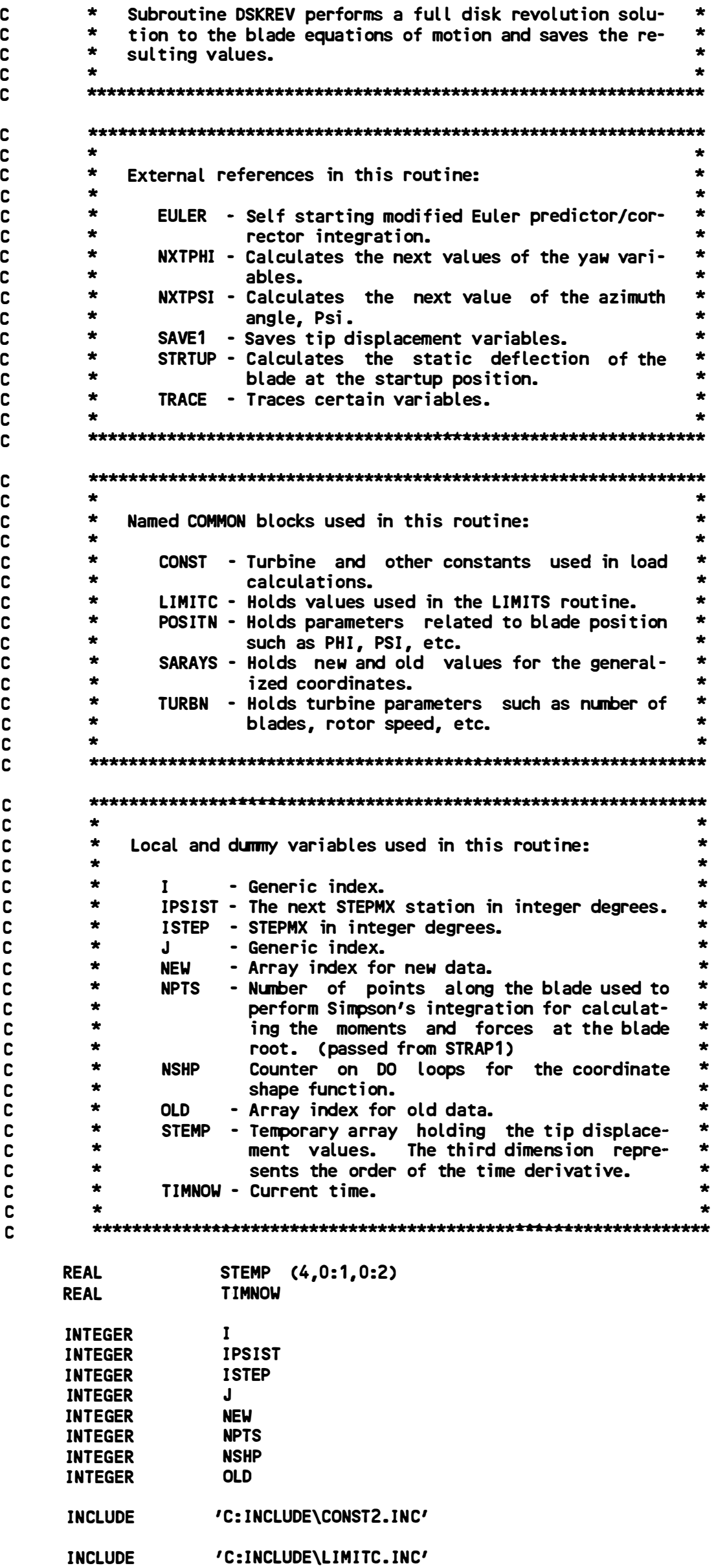




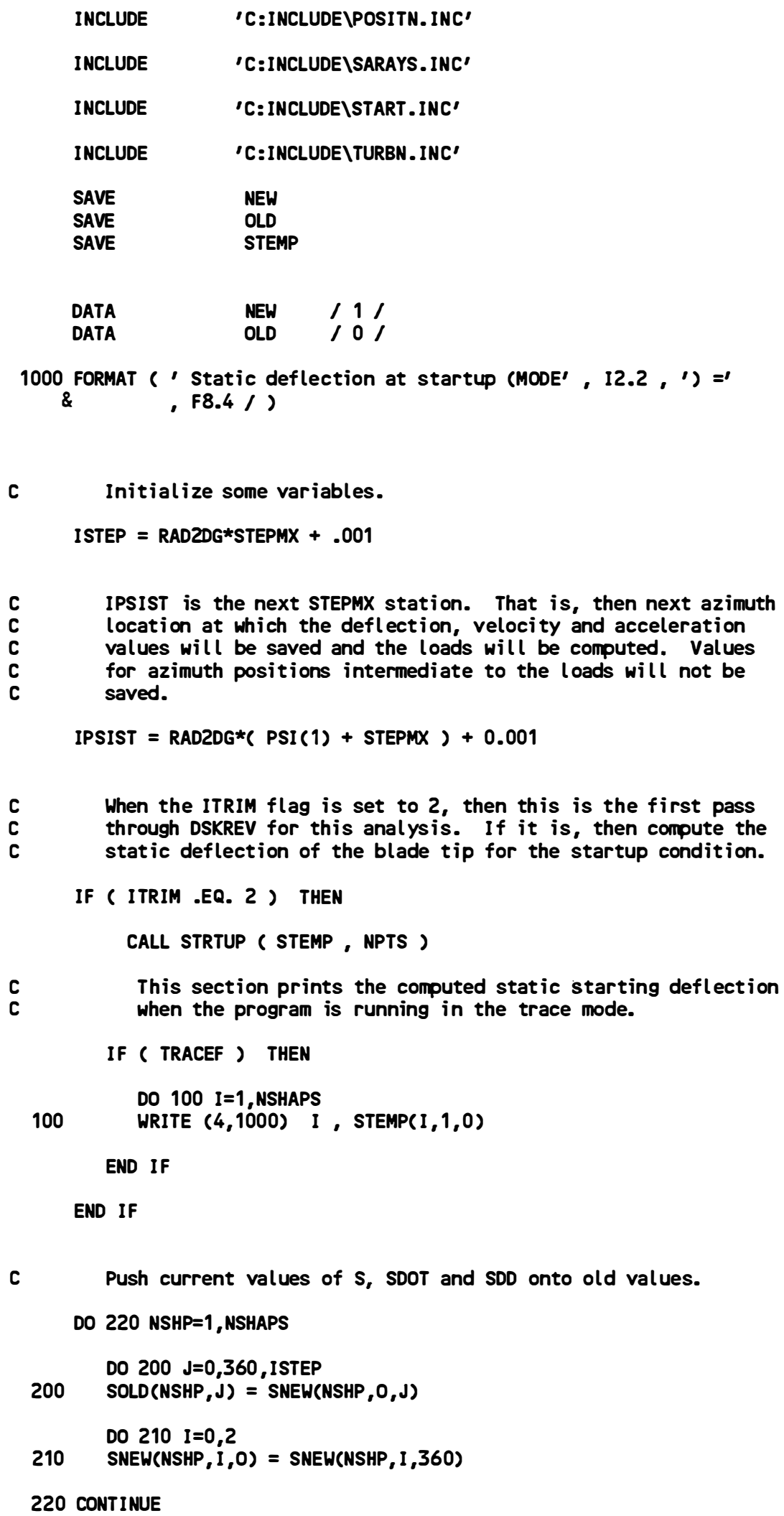


400 CALL NXTPSI ( IPSIST)

$\operatorname{TIMNOW}=\operatorname{TIME}(1)$

CALL NXTPHI ( TIMNOW )

Solve the equation of motion and compute tip displacements and velocities. Check error of Euler predictor-corrector solution. If the error condition is satisfied, check for disk station and save the new values if this azimuth is a STEPMX disk station.

CALL EULER ( STEMP ， NSHAPS ， NPTS )

IF ( (ERROR .GT. EUERR) .AND. (DELPSI(1) . GT. STEPMN) ) GO TO 400

The following tests check for the current azimuth location between the STEPMX stations. If the current position is very close to a STEPMX station, then the deflection, velocity and acceleration values are saved.

IF ( ABS( PSI(1) - IPSIST/RADZDG ) .LT. 0.1*STEPMN ) THEN

CALL SAVE1 ( IPSIST , NSHAPS , STEMP )

IF ( TRACEF ) CALL TRACE ( STEMP , NPTS )

END IF

Move new temporary values to old.

DO 510 NSHP $=1$, NSHAPS

DO $500 \quad I=0,2$

500 STEMP(NSHP,OLD , I ) = STEMP(NSHP, NEW, I )

\section{CONTINUE}

If we haven't gone all the way around, solve the equation of motion for the next step.

IF ( IPSIST .LE. 360 ) GO TO 300

RETURN

END

SUBROUTINE EULER ( STEMP , NSHAPS , NPTS ) 


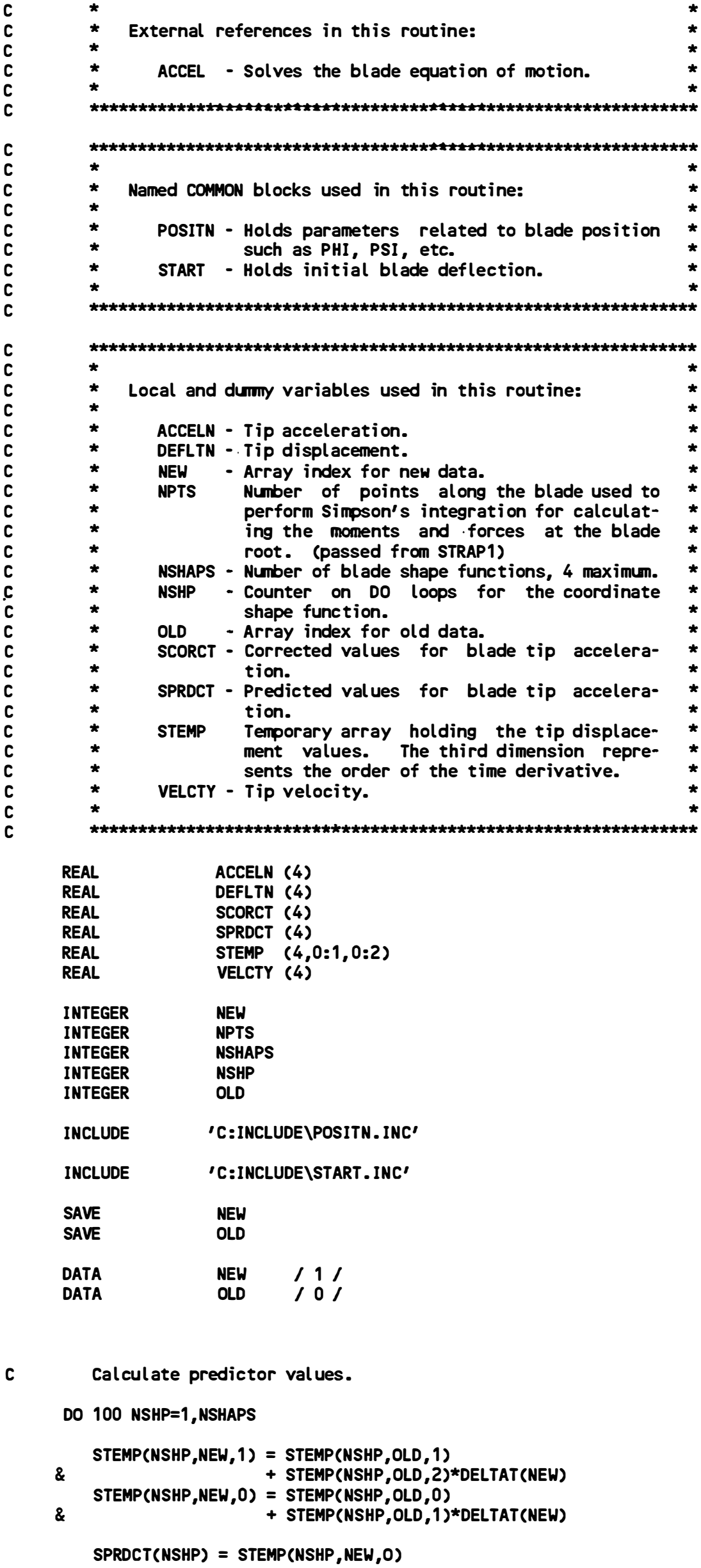
perform Simpson's integration for calculating the moments and forces at the blade root. (passed from STRAP1)

C Calculate predictor values.

DO 100 NSHP $=1$, NSHAPS

$\operatorname{STEMP}(N S H P, N E W, 1)=\operatorname{STEMP}(N S H P, O L D, 1)$

STEMP(NSHP,NEW, 0$)=$ STEMP(NSHP,OLD,0)

\&

+ STEMP(NSHP,OLD,1)*DELTAT(NEW)

SPRDCT(NSHP) $=$ STEMP(NSHP, NEW, O) 
C Calculate predicted acceleration values by solving the blade
C equation of motion with the predicted values of the tip

C velocity and displacement.

DO 200 NSHP $=1$, NSHAPS

DEFLTN(NSHP) $=$ STEMP(NSHP,NEW, 0)

VELCTY(NSHP) $=$ STEMP(NSHP,NEW, 1$)$

200 CONTINUE

C Subroutine ACCEL performs the actual solution of the blade

C equation of motion. It uses the previous values of the tip

C deflection and velocity and the current values of the forces.

C It returns the new blade tip acceleration values.

CALl ACCEl ( DEFLTn , Velcty , ACCELn , NPTS, Stemp )

C

Calculate corrector values.

DO 300 NSHP $=1$, NSHAPS

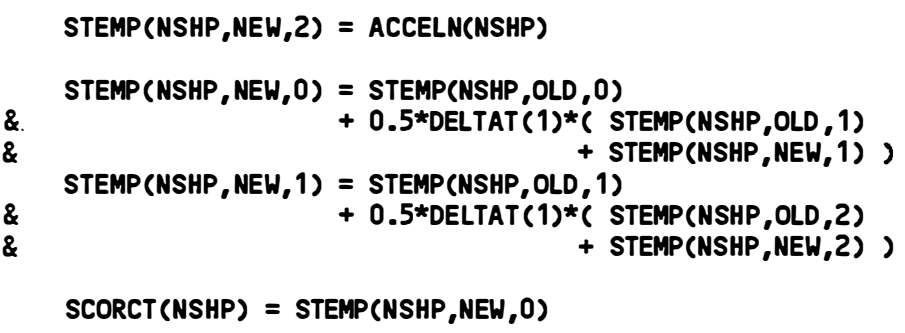

300 CONTINUE C The error is computed from the sum of the ratios of the tip
C displacement change from the predicted to the corrected values relative to the initial static blade deflection computed at startup. Sumation is over the number of coordinate shape functions specified in the run.

$E R R O R=0.0$

DO 400 NSHP $=1$, NSHAPS

400 ERROR $=$ ERROR + ( SCORCT(NSHP) - SPRDCT(NSHP) $) / S O(N S H P)$

C The error value is converted into percent for use in the

C DSKREV routine.

$E R R O R=100.0 * A B S(E R R O R$ )

RETURN

END

SUBROUTINE FORM1 ( STEMP , NPTS , NSHAPS , BLDANG ) 
External references in this routine:

none

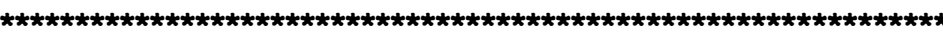

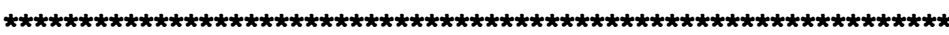

Named COMmON blocks used in this routine:

FORMS - Holds blade deflections.

POSITN - Holds parameters related to blade position * such as PHI, PSI, etc.

SHAPE - Holds blade coordinate shape functions.

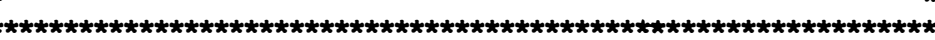

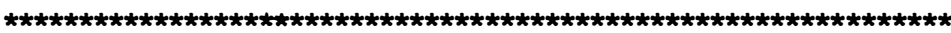

Local and dummy variables used in this routine:

ANGLE1 - Azimuth angle for blade \#1.

ANGLE2 - Azimuth angle for blade \#2.

BLDANG - Blade azimuth position used in the teetering rotor.

I - Generic index.

MARK - Sign of a W term.

NPTS - Number of points along the blade used to perform simpson's integration for calculat- * ing the moments and forces at the blade * root. (passed from STRAP1)

NSHAPS - Number of blade shape functions, 4 maximum.

NSHP - Counter on DO loops for the coordinate shape function.

OLD - Array index for old data.

STEMP - Temporary array holding the tip displacement values. The third dimension represents the order of the time derivative.

\begin{tabular}{|c|c|}
\hline $\begin{array}{l}\text { REAL } \\
\text { REAL } \\
\text { REAL } \\
\text { REAL }\end{array}$ & $\begin{array}{l}\text { ANGLE1 } \\
\text { ANGLE2 } \\
\text { BLDANG } \\
\text { STEMP } \quad(4,0: 1,0: 2)\end{array}$ \\
\hline $\begin{array}{l}\text { INTEGER } \\
\text { INTEGER } \\
\text { INTEGER } \\
\text { INTEGER } \\
\text { INTEGER } \\
\text { INTEGER }\end{array}$ & $\begin{array}{l}\text { I } \\
\text { MARK } \\
\text { NPTS } \\
\text { NSHAPS } \\
\text { NSHP } \\
\text { OLD }\end{array}$ \\
\hline INCLUDE & 'C:INCLUDE \CONST2.INC' \\
\hline INCLUDE & 'C:INCLUDE XFORMS.INC' \\
\hline INCLUDE & 'C:INCLUDEXPOSITN.INC' \\
\hline INCLUDE & 'C:INCLUDE ISHAPE.INC' \\
\hline INCLUDE & 'C: INCLUDE\BLADE2. INC' \\
\hline SAVE & OLD \\
\hline DATA & 101 \\
\hline $\begin{array}{l}\text { Initialize } \\
W(0, I) \text { Is } \\
W(1, I) \text { Is } \\
V 1(0, I) \text { is } \\
V 1(1, I) \text { is } \\
V E(0, I) \text { is } \\
V E(1, I) \text { is }\end{array}$ & $\begin{array}{l}\text { the VV array. } \\
\text { the blade deflection (teeter+elastic) } \\
\text { the blade velocity (teeter+elastic) } \\
\text { the blade teeter deflection } \\
\text { the blade teeter velocity } \\
\text { the elastic part of blade deflection } \\
\text { the elastic part of blade velocity }\end{array}$ \\
\hline
\end{tabular}


DO $100 \mathrm{I}=1$, NPTS

$W(0,1)=0.0$

$W(1,1)=0.0$

$v 1(0,1)=0.0$

$V 1(1,1)=0.0$

$\operatorname{VE}(0,1)=0.0$

$\operatorname{VE}(1, I)=0.0$

100 CONTINUE

$$
\operatorname{VVSLP}(I)=0.0
$$

Compute the WV values down the blade for each of the specified shape functions.

ANGLE1 $=$ PSI $(1)+$ PI

ANGLE2 $=$ PSI (1)

Determine the teeter angle and velocity:

Note that the teeter angle is the total angle

about the teeter pin. The value V1 is the projection

of the teeter deflection and velocity onto the

$X p, Y p, Z p$ system of axes: $V 1=r^{*}$ beta*cos $(d e l t 3)$

*cos(thetap).

BETA $=\operatorname{STEMP}(1,0 L D, 0) /(B L T I P+H U B R A D)$

$B E T A D=\operatorname{STEMP}(1,0 L D, 1) /(B L T I P+H U B R A D)$

IF ( BLDANG .EQ. ANGLEI ) THEN

BETA $=-1 . \star B E T A$

BETAD $=-1 . * B E T A D$

DO $180 I=1$,NPTS

$V 1(0,1)=V 1(0,1)-\operatorname{SHAPE}(1,0,1) * \operatorname{STEMP}(1,0 L D, 0)$

$v 1(1,1)=v 1(1,1)-\operatorname{SHAPE}(1,0,1) * \operatorname{STEMP}(1,0 L D, 1)$

180

CONTINUE

DO 210 NSHP $=1$, NSHAPS

MARK $=(-1) * *$ NSHP

DO $200 \mathrm{I}=1$, NPTS

$\operatorname{VV}(0,1)=W(0,1)+M A R K \star S H A P E(N S H P, 0,1) * S T E M P(N S H P, O L D, 0)$

$\operatorname{VV}(1,1)=W(1,1)+$ MARK $* \operatorname{SHAPE}(N S H P, 0,1) * S T E M P(N S H P, O L D, 1)$

WSLP (I ) =WSLP (I )+MARK *SHAPE (NSHP, 1, I )*STEMP (NSHP, OLD, 0)

200 CONTINUE

210

CONTINUE

C

DETERMINE ONLY THE ELASTIC CONTRIBUTION TO DEFLECTION

AND VELOCITY

DO 650 NSHP $=2$, NSHAPS

MARK $=(-1) * *$ NSHP

DO $640 I=1$, NPTS

- VE $(0,1)=V E(0,1)+M A R K \star S H A P E(N S H P, 0,1) \star S T E M P(N S H P, O L D, 0)$

640

$\operatorname{VE}(1,1)=\operatorname{VE}(1,1)+$ MARK $* \operatorname{SHAPE}(N S H P, 0,1) * \operatorname{STEMP}(N S H P, O L D, 1)$

650 CONT INUE

CONTINUE

ELSE IF ( BLDANG .EQ. ANGLE2 ) THEN

DO $215 I=1$,NPTS

$V 1(0,1)=V 1(0,1)+\operatorname{SHAPE}(1,0,1) * \operatorname{STEMP}(1,0 L D, 0)$

$V 1(1,1)=V 1(1,1)+\operatorname{SHAPE}(1,0,1) * \operatorname{STEMP}(1,0 L D, 1)$

215

CONTINUE

DO 230 NSHP $=1$, NSHAPS

DO $220 I=1$, NPTS 
$W(0,1)=W(0,1)+\operatorname{SHAPE}(N S H P, 0,1) *$ STEMP $(N S H P, O L D, 0)$

$V V(1,1)=W(1,1)+\operatorname{SHAPE}(N S H P, 0,1) * \operatorname{STEMP}(N S H P, O L D, 1)$

$\operatorname{VVSLP}(I)=\operatorname{VSLP}(I)+\operatorname{SHAPE}(N S H P, 1,1) * S T E M P(N S H P, O L D, 0)$

DO 670 NSHP $=2$, NSHAPS

DO $660 I=1$,NPTS

$\operatorname{VE}(0,1)=\operatorname{VE}(0,1)+\operatorname{SHAPE}(N S H P, 0,1) * \operatorname{STEMP}(N S H P, O L D, 0)$

$\operatorname{VE}(1,1)=\operatorname{VE}(1,1)+\operatorname{SHAPE}(N S H P, 0,1) * \operatorname{STEMP}(N S H P, O L D, 1)$

660 CONTINUE

670 CONTINUE

ELSE

PRINT *, $\gg>$ Error in the blade angle, BLDANG, within'

, subroutine FORM1 «<'

PRINT *, , '

PRINT *, , '

PRINT *, 'STRAP terminated abnormally due to the error'

$\&$

' listed above.'

STOP

END IF

RETURN

END

SUBROUTINE GAUSSJ ( A, N )

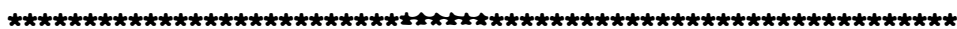

$\star$

* Subroutine GAUSSJ is used to solve linear equations by

* Gauss-Jordan elimination with full pivoting. It was *

* transcribed from the book "Numerical Recipes" by Wil- *

* liam H. Press, et al. Code for right-hand-side vectors *

* was not implemented in this routine. This routine was *

* hard-wired for matrices of maximum order 4.

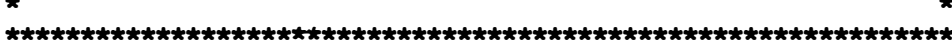

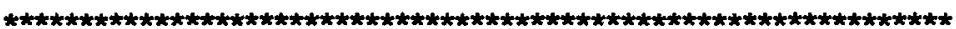
*

* External references in this routine:

* none

*

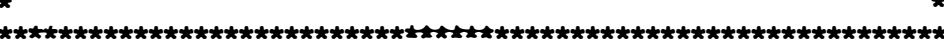

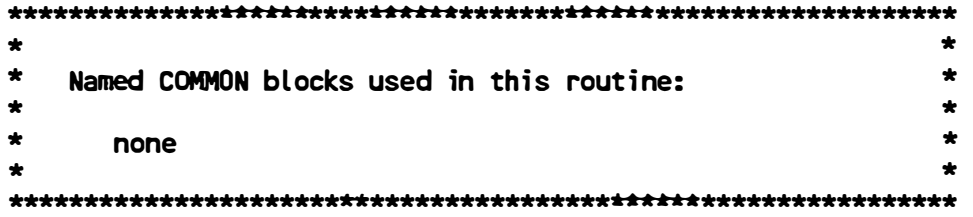

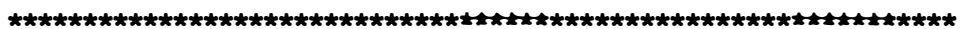

Local and dummy variables used in this routine:

A - Input matrix with NP by NP elements. After processing it is replaced with its inverse.

BIG - The biggest element in the A matrix.

DUM - Temporary storage.

I - Generic index.

ICOL - Column.

INDXC - Used for bookkeeping on pivoting.

INDXR - Used for bookkeeping on pivoting.

IPIV - Used for bookkeeping on pivoting.

$\mathrm{J}$ - Generic index.

IROW - ROW. 


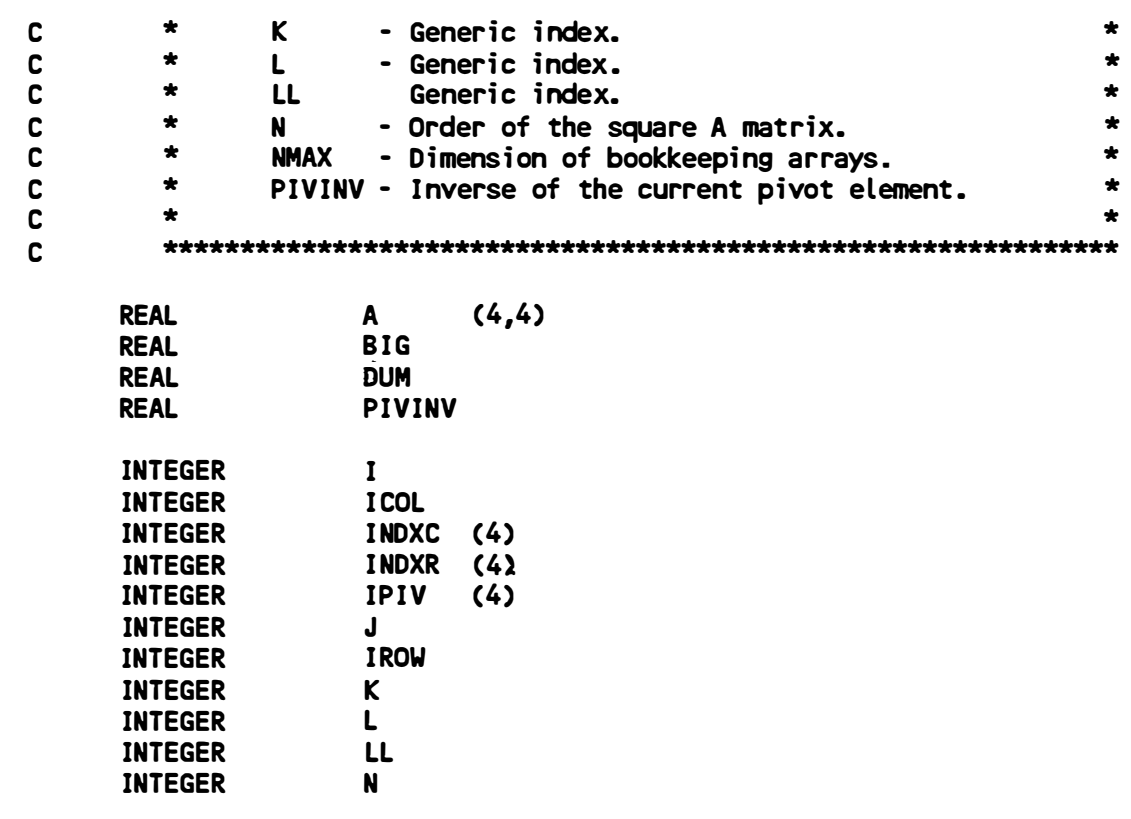

C Initialize the pivot matrix.

DO $100 \mathrm{~J}=1, \mathrm{~N}$

$100 \operatorname{IPIV}(\mathrm{J})=0$

C This is the main loop over the columns to be reduced.

DO $260 \mathrm{I}=1, \mathrm{~N}$

BIG $=0.0$

C

This is the outer loop of the search for a pivot element.

DO $210 \mathrm{~J}=1, \mathrm{~N}$

IF ( IPIV(J) .NE. 1 ) THEN

DO $200 K=1, N$

IF ( IPIV(K) .EQ. 0 ) THEN

IF ( $A B S(A(J, K))$.GE. BIG) THEN

$B I G=A B S(A(J, K))$

IROW $=\mathrm{J}$

$I C O L=K$

END IF

ELSE IF ( IPIV(K) .GT. 1 ) THEN

PRINT *, 1

PRINT *, , \> The input matrix to GAUSSJ is

\&

' 'singular. $\ll<1$

PRINT *, 1 ,

PRINT *, 'STRAP terminated abnormally due to the '

, 'the error listed above.'

STOP

END IF

200

CONT INUE

END IF 
We now have the pivot element, so we interchange rows. If needed, to put the pivot element on the diagonal. The colums are not physically interchanged, only relabeled: INDXC(I), the colum of the Ith pivotal element, is the Ith colum that is reduced, while INDXR(I) is the row in which that pivot element was originally located. If INDXR(I) is not equal to INDXC(I), there is an implied column interchange. With this form of bookkeeping, the solution Bs will end up in the correct order, and the inverse matrix will be scrambled by colums.

IF ( IROW .NE. ICOL ) THEN

DO $220 L=1, N$

$$
\begin{array}{ll}
\text { DUM } & =A(I R O W, L) \\
A(I R O W, L) & =A(I C O L, L) \\
A(I C O L, L) & =\text { DUM }
\end{array}
$$

END IF

$\operatorname{INDXR}(I)=I R O W$

$\operatorname{INDXC}(I)=I C O L$

IF (. A(ICOL,ICOL) .EQ. 0.0$)$ THEN

\&

PRINT *, , ,

PRINT *, , \> The input matrix to GAUSSJ is '

'singular. $\ll<'$

PRINT *, , '

\&

PRINT *', 'STRAP terminated abnormally due to the ' , 'the error listed above.'

STOP

END IF

PIVINV $=1.0 / \mathrm{A}(I C O L, I C O L)$

$A(I C O L, I C O L)=1.0$

DO $230 L=1, N$

$230 \quad A(I C O L, L)=$ PIVINV*A $(I C O L, L)$

C

Reduce all the rows except the pivot one.

DO $250 L L=1, N$

IF ( LL .NE. ICOL ) THEN

DUM $\quad=A(L L, I C O L)$

$A(L L, I C O L)=0.0$

240

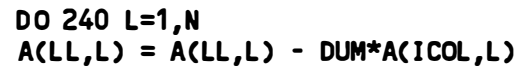

END IF

250

CONTINUE

260 CONTINUE

C Unscramble the solution in view of the colum interchanges.

C We do this by interchanging pairs of columns in the reverse

C order that the permutation was built up.

DO $310 L=N, 1,-1$ 
IF ( $\operatorname{INDXR}(L)$.NE. INDXC(L) ) THEN

DO $300 K=1, N$

$$
\begin{array}{ll}
\operatorname{DUM} & =A(K, \operatorname{INDXR}(L)) \\
A(K, \operatorname{INDXR}(L)) & =A(K, \operatorname{INDXC(L))} \\
A(K, \operatorname{INDXC}(L)) & =\operatorname{DUM}
\end{array}
$$

300

CONTINUE

END IF

310 CONTINUE

RETURN

END

SUBROUTINE GETFIL ( RESFIL )

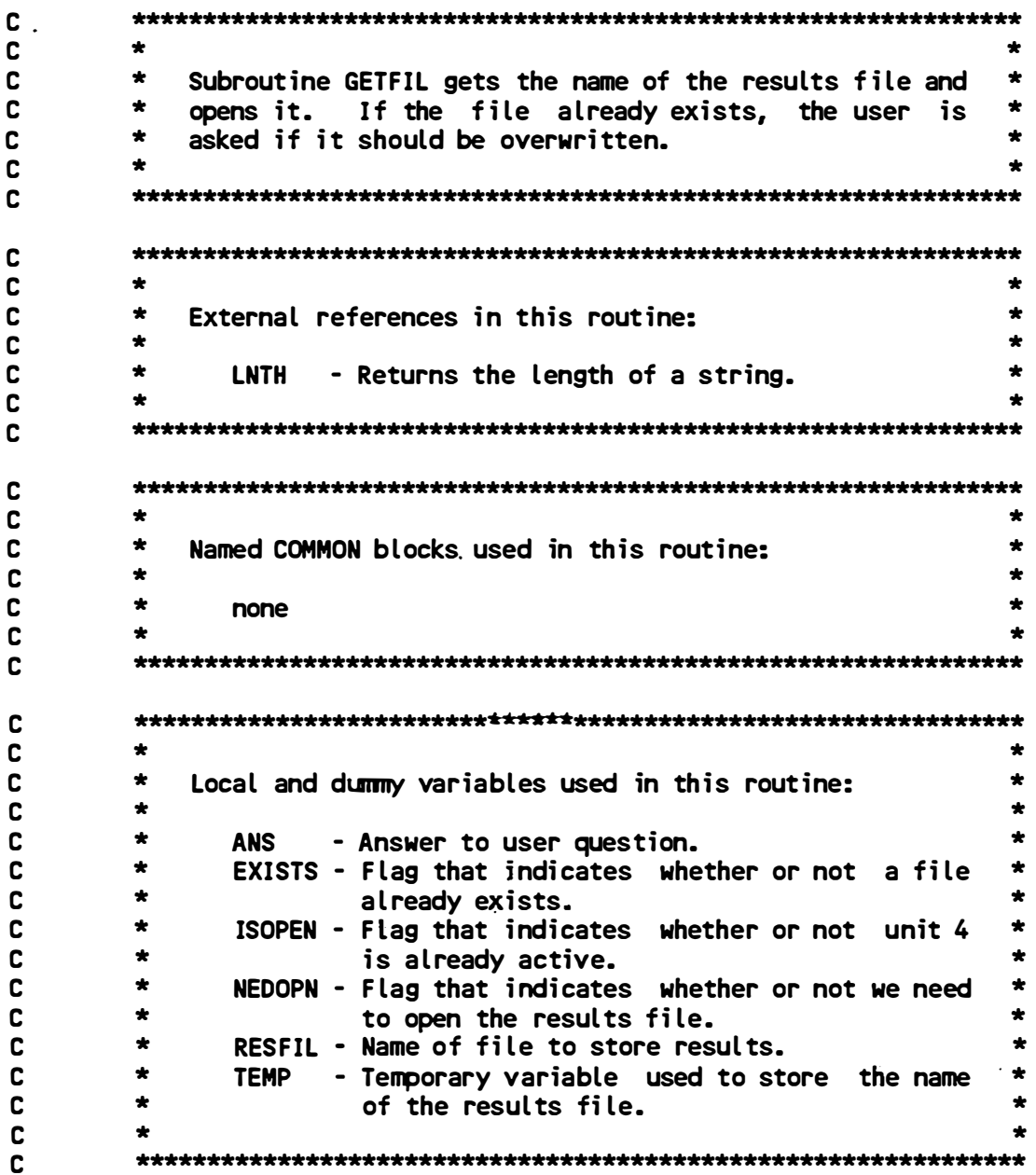

$\begin{array}{ll}\text { INTEGER } & \text { LNTH } \\ \text { LOGICAL } & \text { EXISTS } \\ \text { LOGICAL } & \text { ISOPEN } \\ \text { LOGICAL } & \text { NEDOPN } \\ & \\ \text { CHARACTER*1 } & \text { ANS } \\ \text { CHARACTER*(*) } & \text { RESFIL } \\ \text { CHARACTER*50 } & \text { TEMP }\end{array}$

1000 FORMAT ('Enter name of results file $[=\prime, A, ']>$ ')

1100 FORMAT ( A )

1200 FORMAT ('1') 
C

Get name of results file.

100 PRINT 1000, RESFIL(1:LNTH(RESFIL))

READ 1100, TEMP

IF ( TEMP .EQ. ' , ) THEN

C

The user hit just an <Enter>. Use the old name.

TEMP = RESFIL

ELSE

C

Convert file name to upper case if a name was typed in.

CALL CAPS ( TEMP )

END IF

C

Check to see if unit 4 is already attached.

INQUIRE ( 4, OPENED=ISOPEN )

IF ( ISOPEN ) THEN

IF ( TEMP .EQ. RESFIL ) THEN

C Use the same file that's already open. Eject a page.

WRITE $(4,1200)$

NEDOPN $=$. FALSE.

ELSE

C Use a different file. Close old one.

CLOSE ( 4 ) NEDOPN $=$.TRUE.

END IF

ELSE

C No open file yet. We need to open one. NEDOPN $=$.TRUE.

END IF

IF ( NEDOPN ) THEN

C We need to open the new results file. Does it already exist?

INQUIRE ( FILE=TEMP , EXIST=EXISTS )

IF ( EXISTS ) THEN

C File already exists. Write over it?

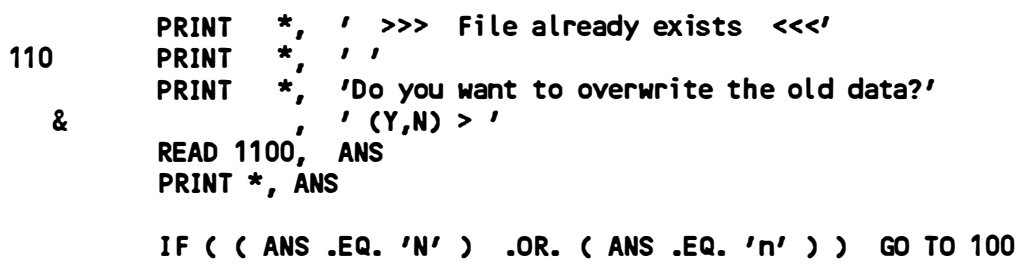


IF ( ( ANS .NE. 'Y') .AND. ( ANS .NE. ' $Y$ ' ) ) GO TO 110

END IF

Open the results file.

\&

OPEN ( 4 , FILE=TEMP , STATUS='UNKNOWN'

- CARRIAGECONTROL='FORTRAN')

END IF

C

Save name of results file.

RESFIL = TEMP

RETURN

END

SUBROUTINE INDUCD ( INBORD , NPTS , BLDANG )

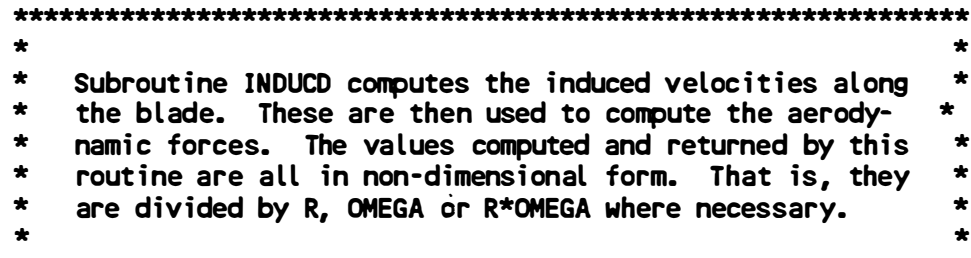

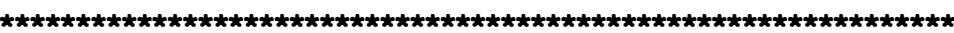

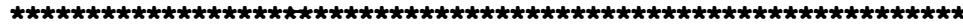

External references in this routine:

none

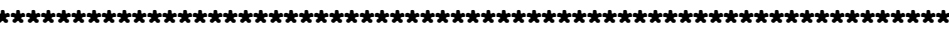

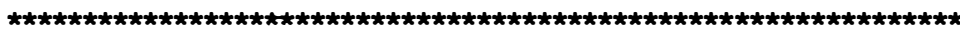

Named COMMON blocks used in this routine:

AER01 - Holds coefficients related to aerodynamic loads calculations such as ClAlpha, Cdzero, etc.

BLADE - Holds blade property values such as stiffness and mass distributions.

CONST - Turbine and other constants used in load calculations.

POSITN - Holds parameters related to blade position such as PHI, PSI, etc.

TURBN - Holds turbine parameters such as number of blades, rotor speed, etc.

VINDUC - Holds induced velocity components.

WIND - Holds wind shear and tower shadow parameters.

WNDVEL - Holds values used in wind shear and tower shadow computations.

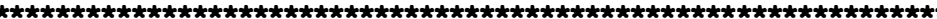

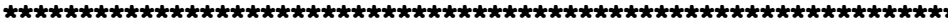

Local and dumy variables used in this routine:

$\begin{array}{lll}\text { A2BVL - Temporary storage. } & * \\ \text { AA } & \text { - Temporary storage. } \\ \text { ALMP } & * \text { Temporary storage. } \\ \text { AM2BV - Temporary storage. } & * \\ \text { AMBV - Temporary storage. } & * \\ \text { APR - Temporary storage. } & * \\ \text { APRIME - Temporary storage. } & * \\ \text { ASTAR - Temporary storage. } & * \\ \text { BB } & * \text { Temporary storage. } \\ \text { BLDANG - Blade azimuth position used in the teeter- }\end{array}$




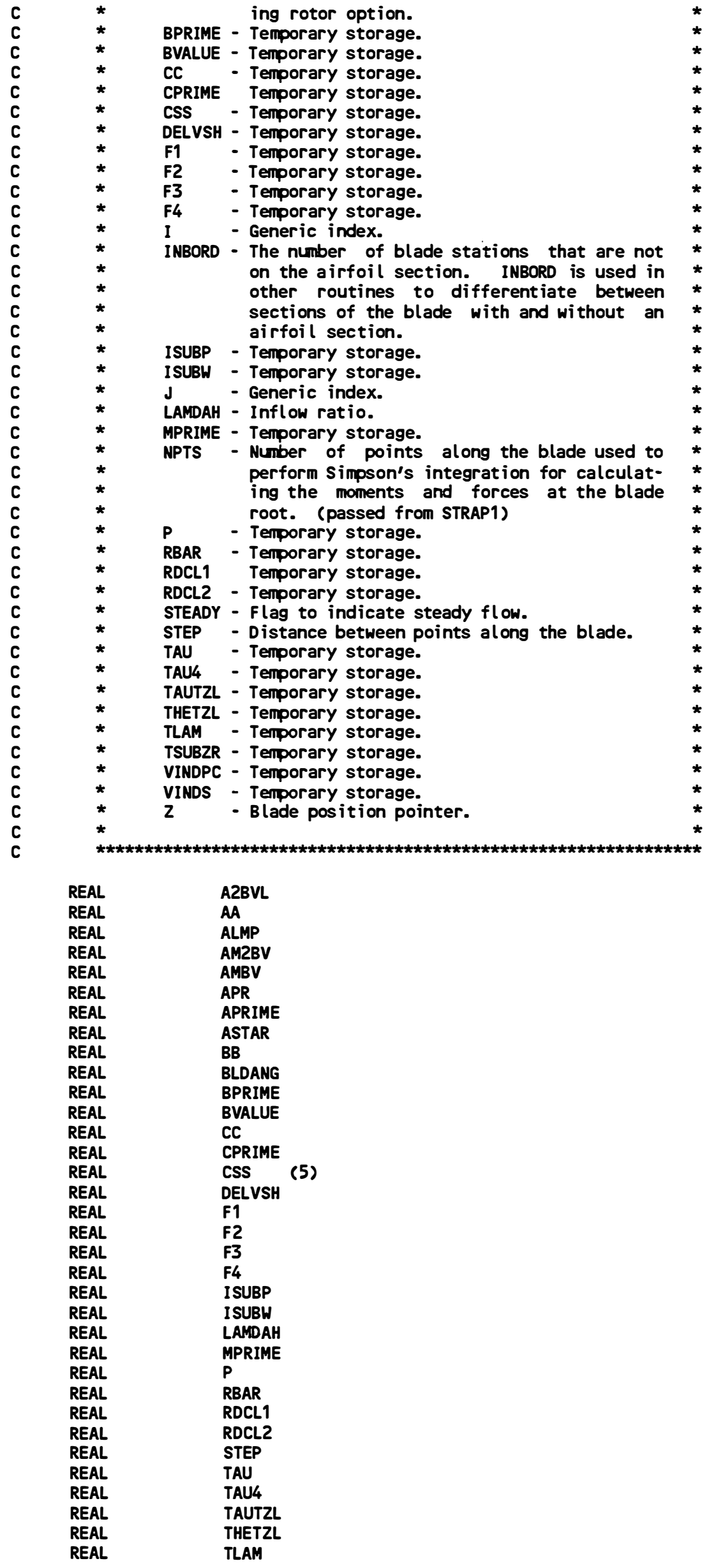




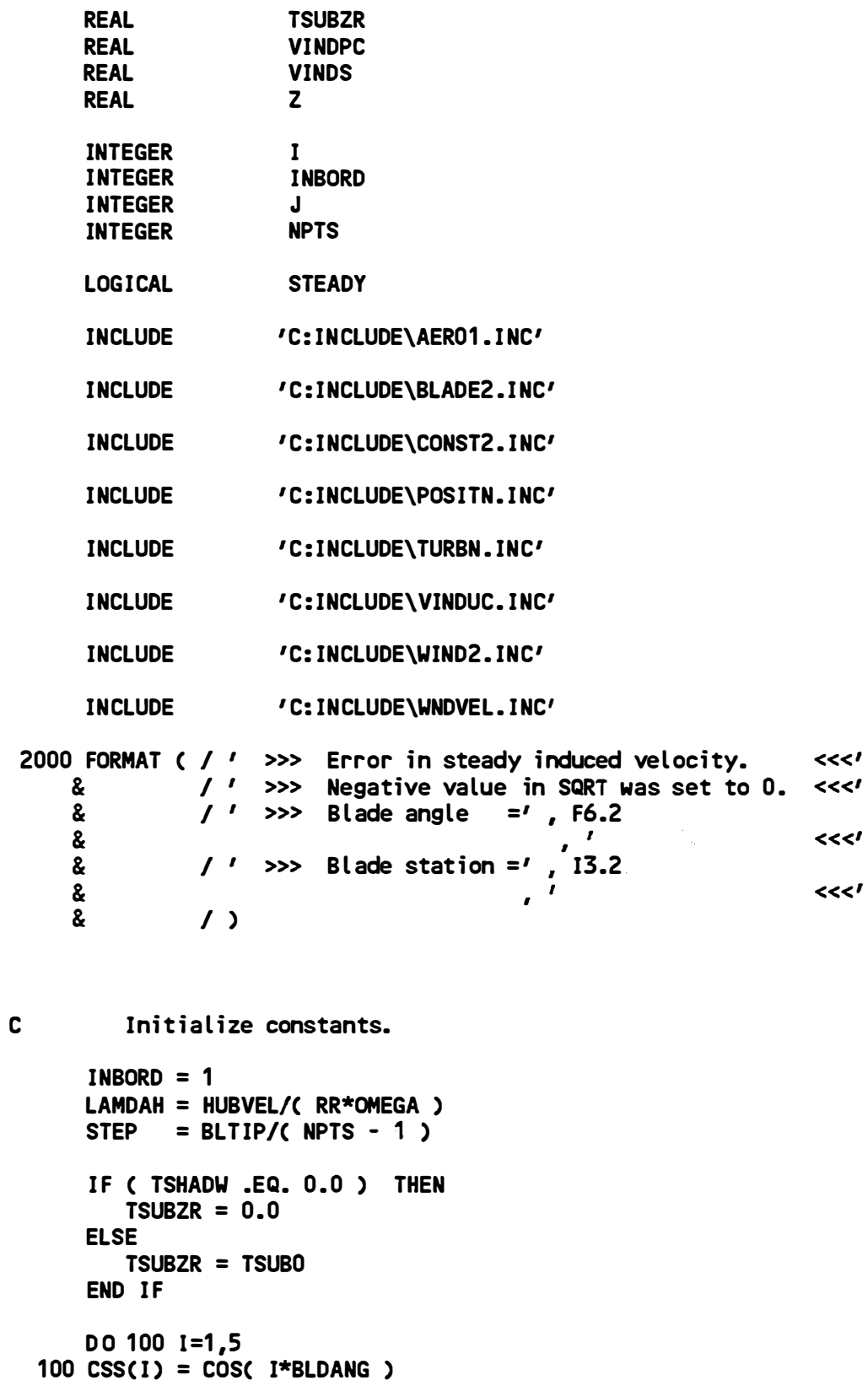

C Move blade station pointer to inboard start of airfoil section 


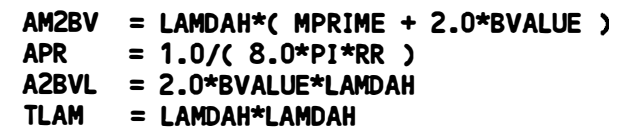

DO $220 I=1$,NPTS

IF ( I . LT. INBORD ) THEN

$$
\begin{aligned}
& \operatorname{VINDR}(I)=0.0 \\
& \operatorname{VINDO}(I)=0.0 \\
& \operatorname{DVIND}(I)=0.0
\end{aligned}
$$

ELSE

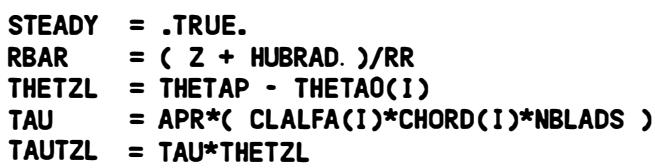
the equation is linear. If the radical of the root is less than zero, then the momentum solution is invalid, set the radical to zero and go on.

IF ( AA .NE. 0.0) THEN

RDCL1 $=B B * * 2-4.0 * A A * C C$

IF ( RDCL1 .LT. 0.0 ) RDCL1 $=0.0$

$\operatorname{VINDR}(I)=0.5 *(-B B+\operatorname{SQRT}(\operatorname{RDCL} 1)) / A A$

ELSE

$\operatorname{VINDR}(I)=-C C / B B$

END IF

IF ( ( 1.0-VINDR(I)/LAMDAH .GT. ASTAR ) .OR.

Check to see if the induced condition is steady or turbulent. If it is turbulent, compute turbulent intermediate values and recompute the induced velocity.

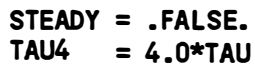

APRIME $=2.0 *$ TAUTZL BPRIME = -RBAR*( ALMP + TAU4 )

- LAMDAH*( WSHR(O,I) - TSUBZR )

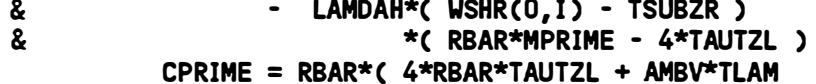
*( AM2BV - TAU4 ) ) for steady flow.

IF ( APRIME .NE. 0.0 ) THEN

RDCL2 $=$ BPRIME*ะ2 - 4*APRIME*CPRIME

IF ( RDCL2 .LT. 0.0 ) - THEN 
PRINT 2000, BLDANG , I

RDCL2 $=0.0$

END IF

$\operatorname{VINDR}(I)=-0.5 *($ BPRIME + SQRT( RDCL2 ) )/APRIME

ELSE

$\operatorname{VINDR}(I)=-C P R I M E / B P R I M E$

END IF

END IF

$\operatorname{VINDO}(I)=$ LAMDAH $-\operatorname{VINDR}(I)$

Calculate the intermediate components for forming induced velocity terms.

F1 $=$ TAU*VINDR(I $) *(\operatorname{VINDR}(1)-2 *$ RBAR*THETZL $)$

$F 2=$ TAU* ( RBAR - THETZL*VINDR(I) )

$F 3=$ RBAR*( VINDR(I) $-\operatorname{VINDO}(I))+F 2$

IF ( STEADY ) THEN

ISUBP $=F 1 / F 3$

ISUBW $=$ LAMDAH*( F2 - RBAR*VINDO(I) $) / F 3$

ELSE

$F 4=$ RBAR*ALMP $+4 * F 2$

ISUBP $=4 * F 1 / F 4$

ISUBW $=$ LAMDAH*C $4 * F 2-R B A R * C$ A2BVL

\&

END IF

Form induced velocity components of order epsilon, etc.

$\operatorname{VINDC}(1)=-P H I(0) * I S U B P+\operatorname{WSHR}(1,1) * I S U B W$

VINDS $=-$ CHI*ISUBP

$\operatorname{VINDC}(\mathrm{J})=\operatorname{WSHR}(\mathrm{J}, \mathrm{I}) * I$ SUBW

C

Compute the tower shadow effect if in the tower shadow region.

IF ( TSHADW .NE. 0.0 ) THEN

VINDPC $=$ TSUBP*ISUBW

P $=$ KSHADW*PI/PSIZER

DELVSH $=$ VINDPC $* \operatorname{COS}(P *($ BLDANG - PSISHD ) $)$

ELSE

VINDPC $=0.0$

DELVSH $=0.0$

END IF

C

Compute the delta-V-induced term. i.e., the sum of the order epsilon components.

DVIND(I) $=$ VINDS*SIN( BLDANG ) - DELVSH

210

DO $210 \mathrm{~J}=1,5$

$\operatorname{DVIND}(I)=\operatorname{DVIND}(I)+\operatorname{VINDC}(\mathrm{J}) * \operatorname{css}(\mathrm{J})$

END IF

D-44 
$Z=Z+$ STEP

RETURN

END

SUBROUT INE INVERT ( AMAT , AINV , N )

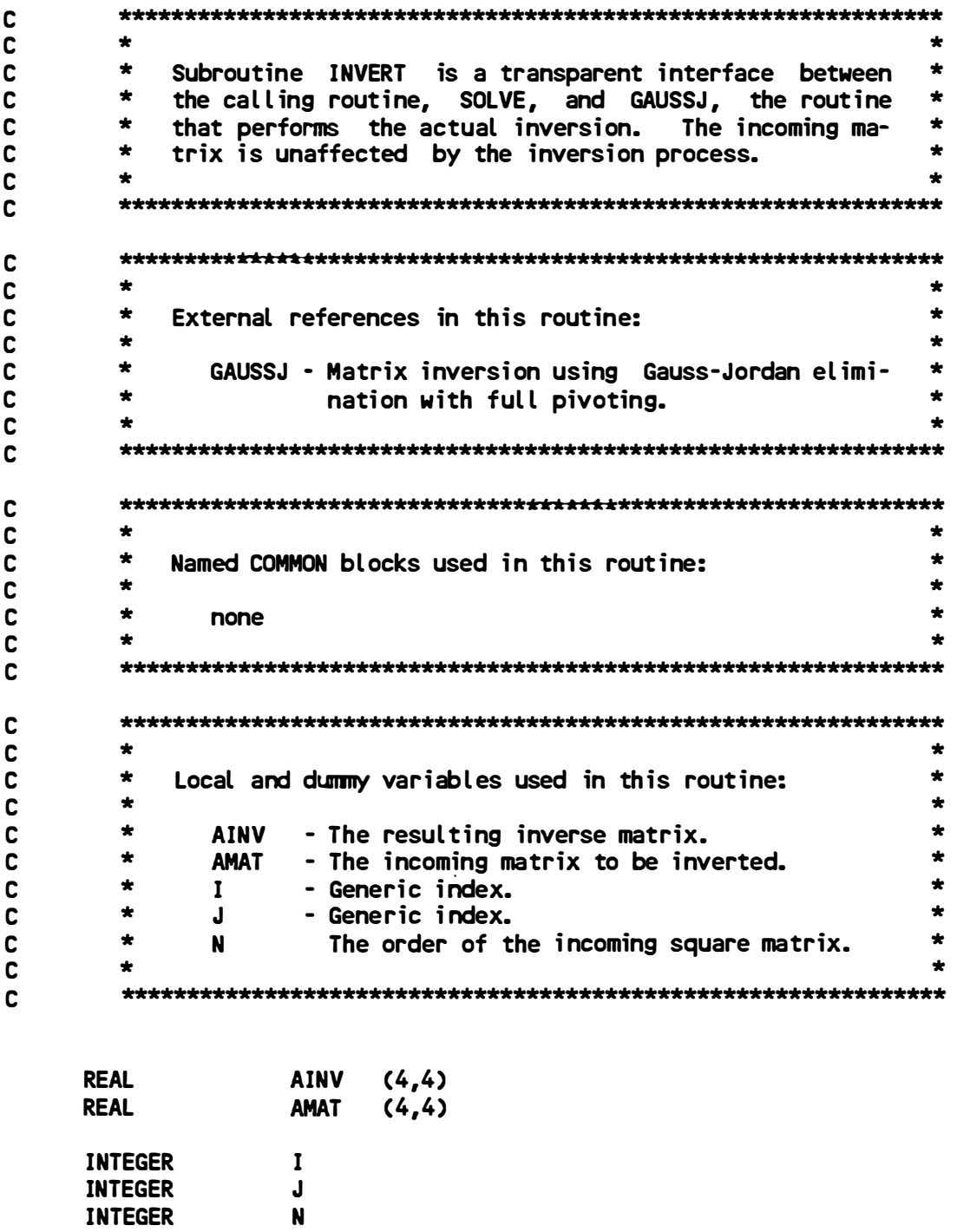

C Load the incoming matrix into the inverse matrix AINV.

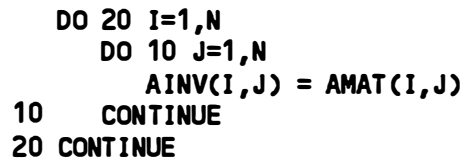

C Invert the matrix.

CALL GAUSSJ ( AINV , N ) 


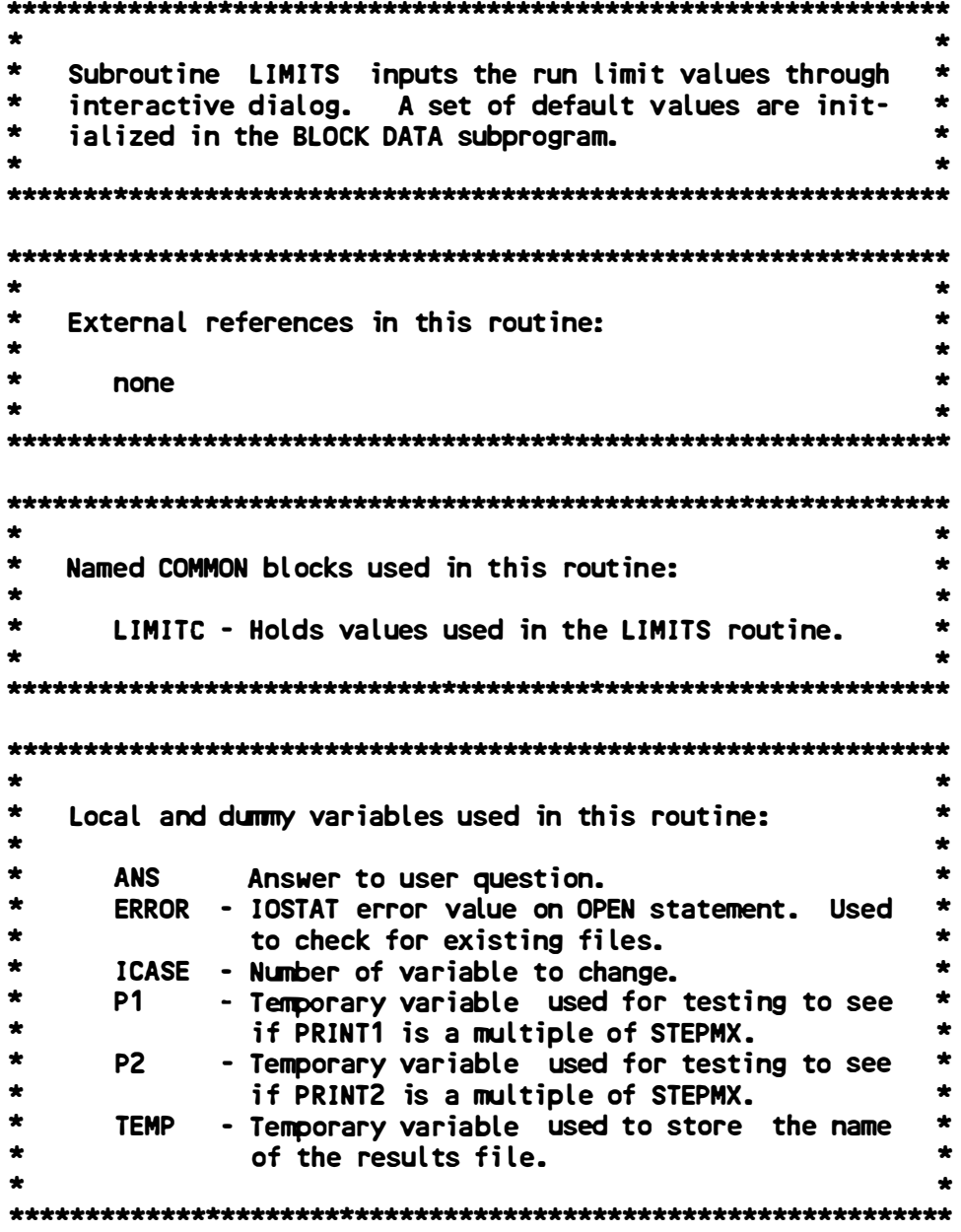

REAL P1

REAL P2

INTEGER ICASE

CHARACTER*1 ANS

INCLUDE 'C:INCLUDEILIMITC.INC'

1000 FORMAT ( // ' The current values of the run parameters are:' / )

1100 FORMAT ( $1 X, A$, F8.3 , A )

1200 FORMAT ( $1 X, A, 14,4 X, A)$

2000 FORMAT ( A )

C Print out the current limit values.

100 PRINT 1000

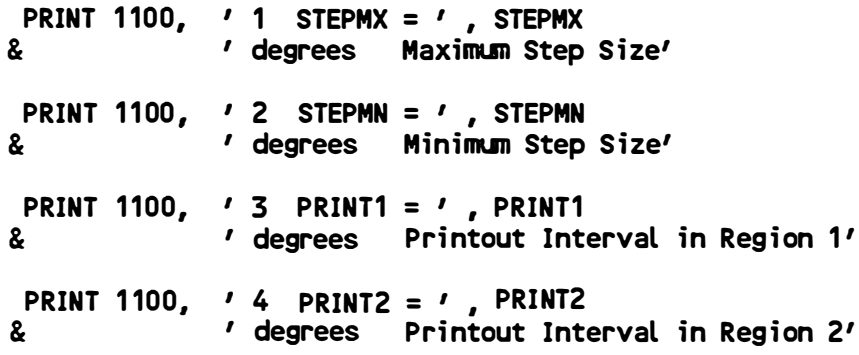




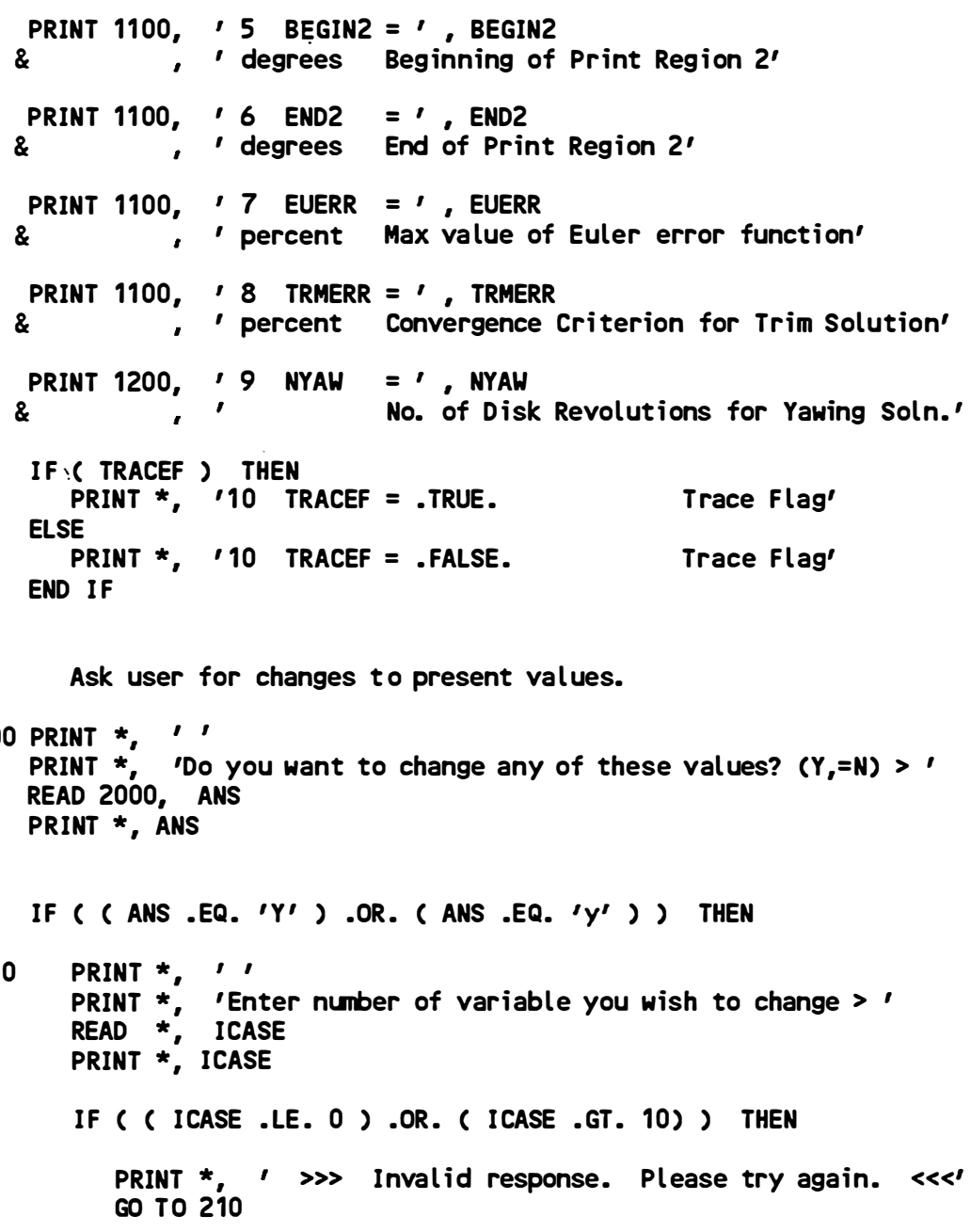

ELSE

C

Change one of the variables.

Go TO $(300,310,320,330,340,350,360,370,380,390)$, ICASE
300 PRINT *, 'Enter new REAL value for STEPMX > '
READ *, STEPMX
PRINT *, STEPMX
GO TO 100
310 PRINT *, 'Enter nEw REAL value for STEPMN > '
READ *, STEPMN
PRINT * STEPMN
GO TO 100

320 PRINT *, 'Enter new REAL value for PRINT1 > '

READ *, PRINT1

PRINT *, PRINT1

GO TO 100

330 PRINT *, 'Enter nEw REAL value for PRINT2 > '

READ *, PRINT2

PRINT *, PRINT2

GO TO 100

340 PRINT *, 'Enter new REAL value for BEGIN2 > '

READ *, BEGIN2

PRINT *, BEGIN2

GO TO 100

350 PRINT *, 'Enter new REAL value for END2 > '

READ *, END2

PRINT *, END2 
GO TO 100

360 PRINT *, 'Enter nEw REAL value for EUERR > '

READ * EUERR

PRINT *, EUERR

GO TO 100

370 PRINT *, 'Enter nEw REAL value for TRMERR > '

READ *, TRMERR

PRINT *, TRMERR

GO TO 100

380 PRINT *, 'Enter new INTEGER value for NYAW > '

READ *, NYAW

PRINT * NYAW

GO TO 100

390

PRINT *, 'Enter new LOGICAL value for TRACEF $(T, F)>$ '

READ *', TRACEF

PRINT * TRACEF

GO TO 100

END IF

ELSE IF ( ( ANS .NE. 'N') . AND. ( ANS .NE. ' $n$ ')

PRINT *, , \> Invalid response. Please try again. «<।

GO TO 200

END IF

C

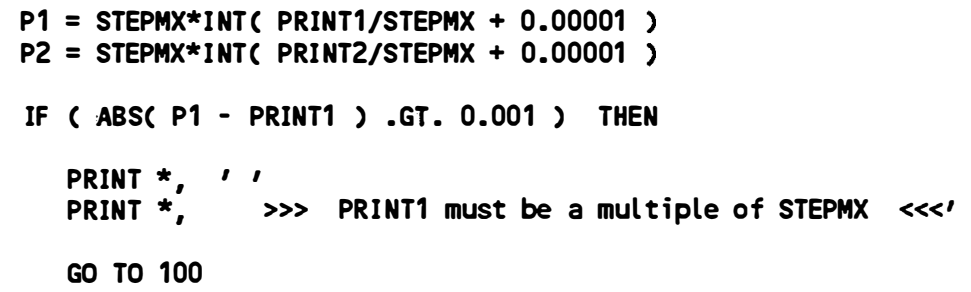

END IF

IF ( ABS( P2 - PRINT2 ) .GT. 0.001 ) THEN

PRINT *, , ,

PRINT *, , $\gg>$ PRINT2 must be a multiple of STEPMX $\ll<1$

GO TO 100

END IF

RETURN

END

FUNCTION LNTH ( STRING )

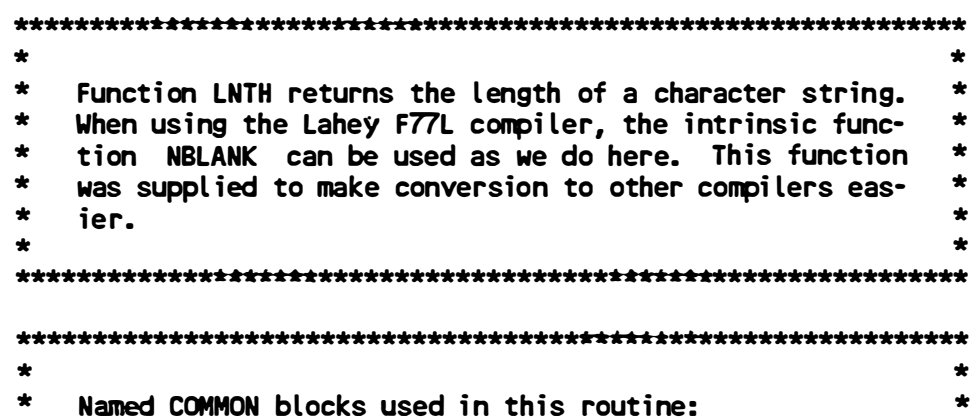




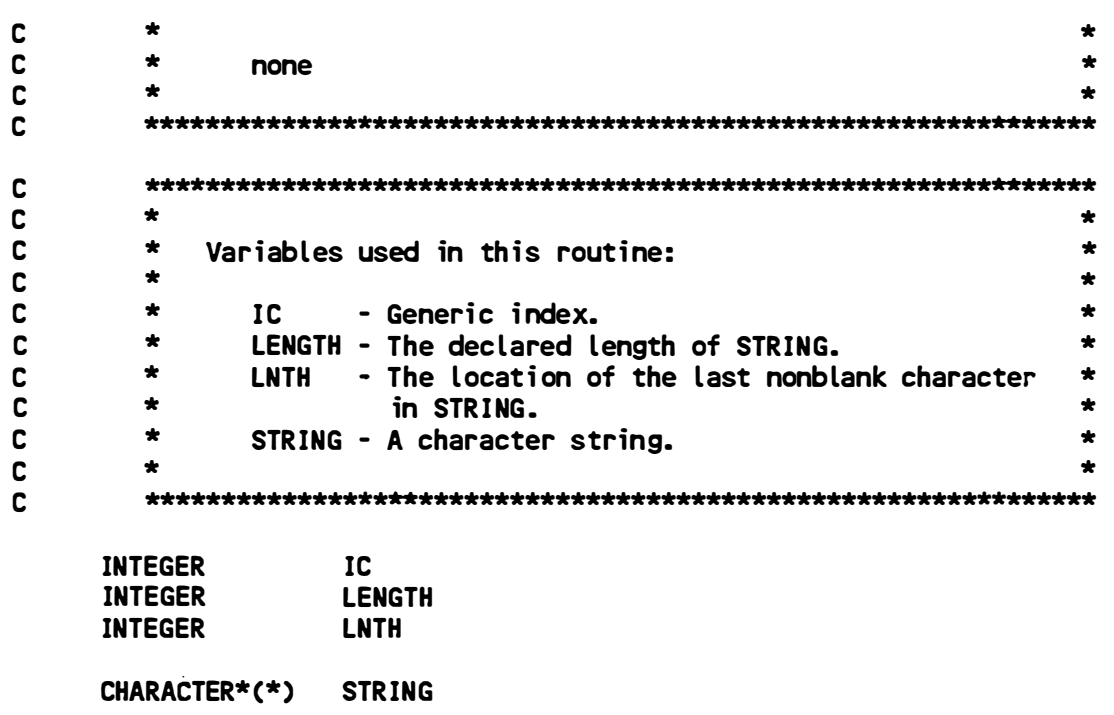

c Get the declared length of STRING using the FORTRAN 7 intrinsic C function LEN.

LENGTH = LEN( STRING )

C Find the location of the last nonblank character in STRING.

DO 100 IC=LENGTH, 1,-1

LNTH = IC

IF ( STRING(IC:IC) .NE. ' I ) GO TO 200

100 CONTINUE

C STRING is all blanks.

LNTH $=0$

200 RETURN

END

SUBROUTINE LODOUT ( NCALLS )

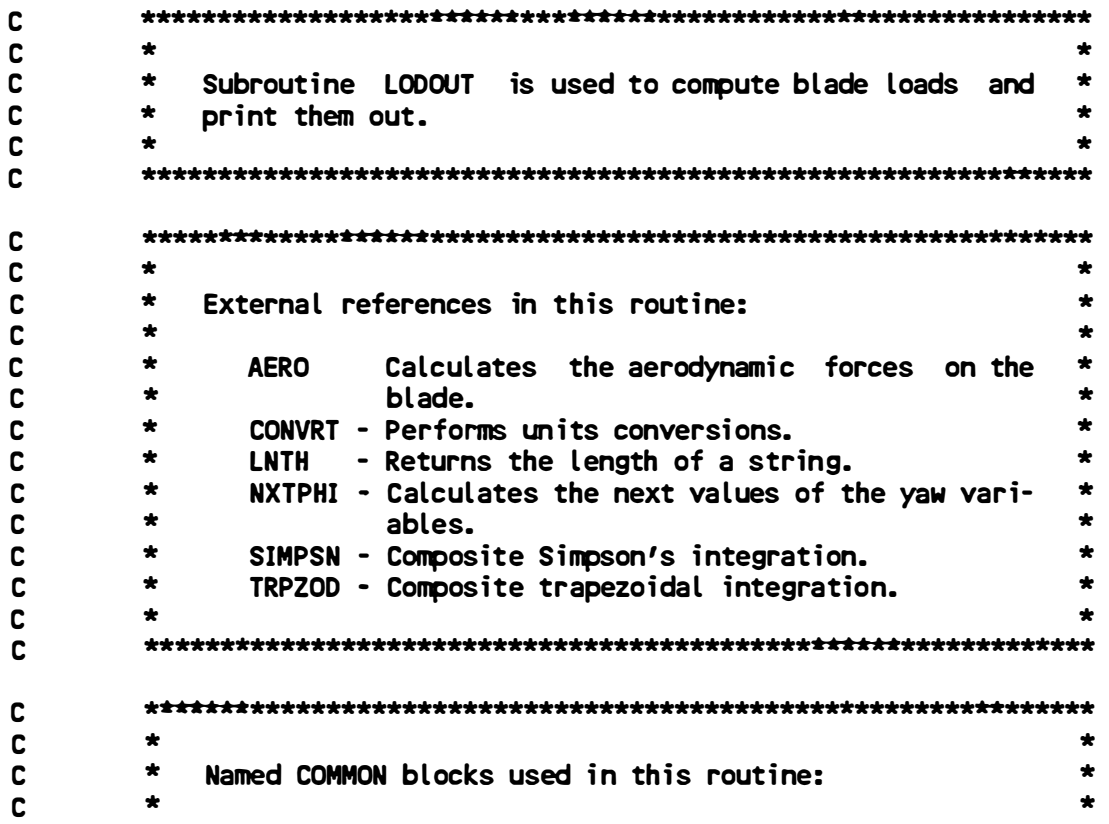




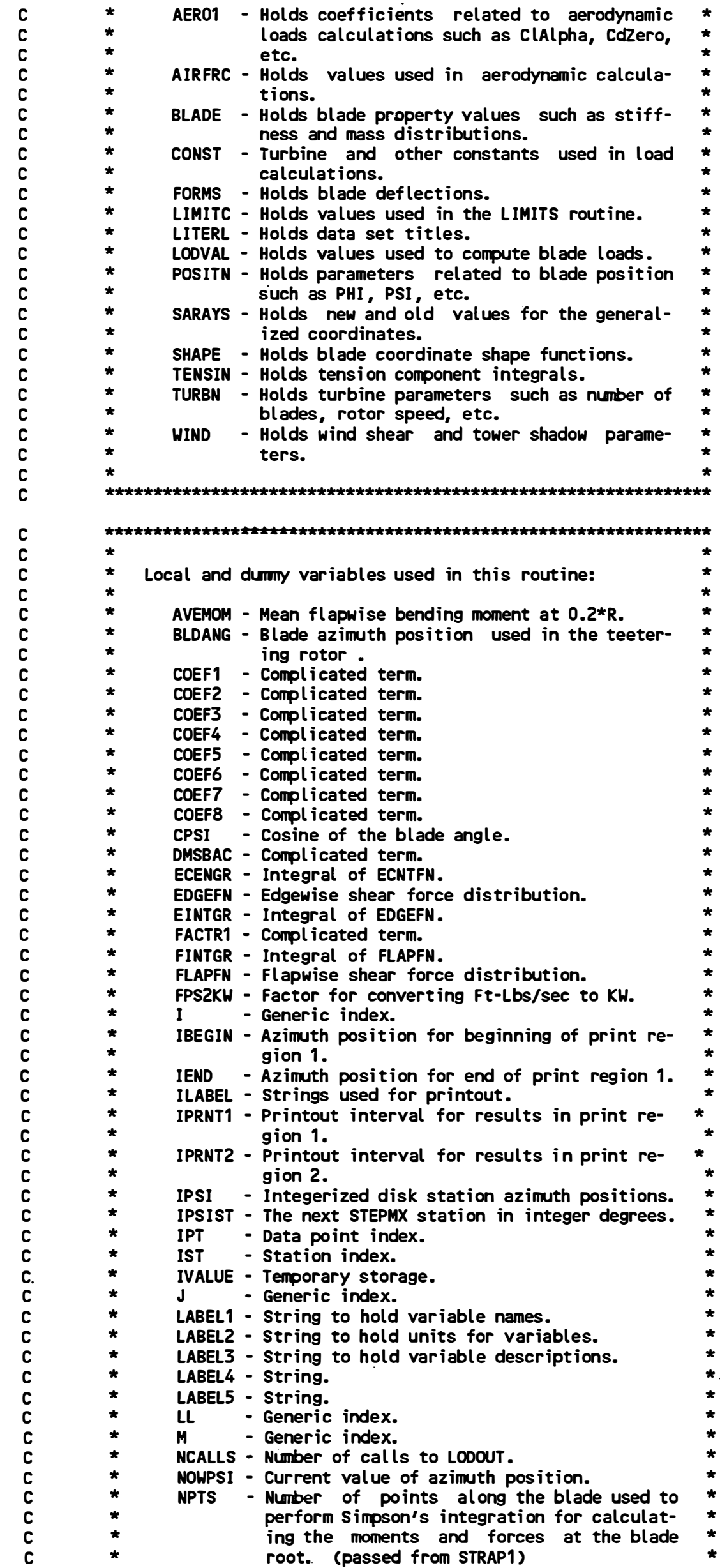




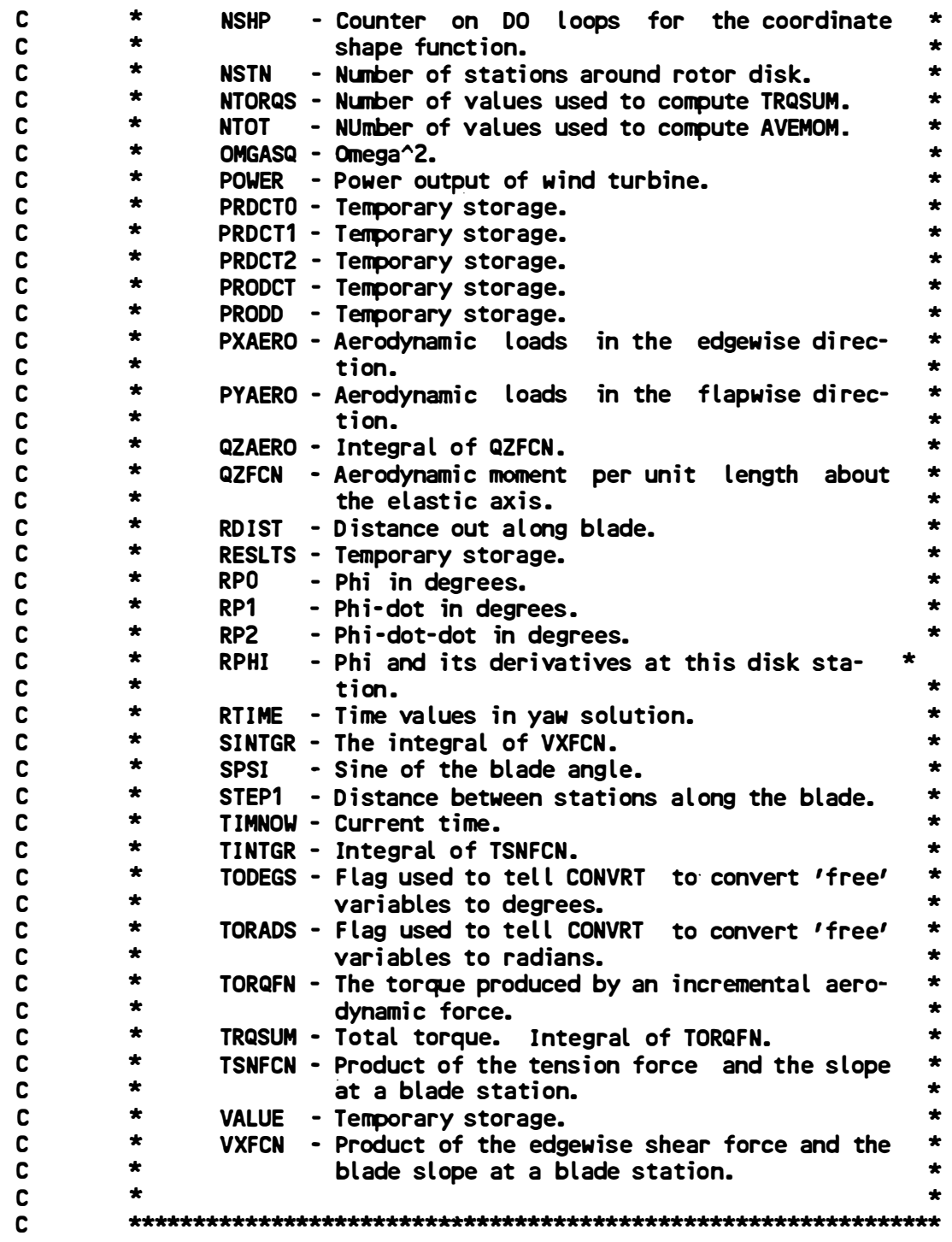

$\begin{array}{ll}\text { REAL } & \text { AVEMOM } \\ \text { REAL } & \text { BLDANG } \\ \text { REAL } & \text { COEF1 } \\ \text { REAL } & \text { COEF2 } \\ \text { REAL } & \text { COEF3 } \\ \text { REAL } & \text { COEF4 } \\ \text { REAL } & \text { COEF5 } \\ \text { REAL } & \text { COEF6 } \\ \text { REAL } & \text { COEF7 } \\ \text { REAL } & \text { COEF8 } \\ \text { REAL } & \text { CPSI } \\ \text { REAL } & \text { DAMCOF } \\ \text { REAL } & \text { DMSBAC } \\ \text { REAL } & \text { ECENGR } \\ \text { REAL } & \text { EDGEFN (21) } \\ \text { REAL } & \text { EINTGR } \\ \text { REAL } & \text { FACTR1 } \\ \text { REAL } & \text { FINTGR } \\ \text { REAL } & \text { FLAPFN (21) } \\ \text { REAL } & \text { FDAMP (360) } \\ \text { REAL } & \text { FPS2KW } \\ \text { REAL } & \text { OMGASQ } \\ \text { REAL } & \text { POWER } \\ \text { REAL } & \text { PRDCTO } \\ \text { REAL } & \text { PRDCT1 } \\ \text { REAL } & \text { PRDCT2 } \\ \text { REAL } & \text { PRODCT (21) } \\ \text { REAL } & \text { PRODD } \\ \text { REAL } & \text { PXAERO (21) } \\ \text { REAL } & \text { PYAERO (21) }\end{array}$




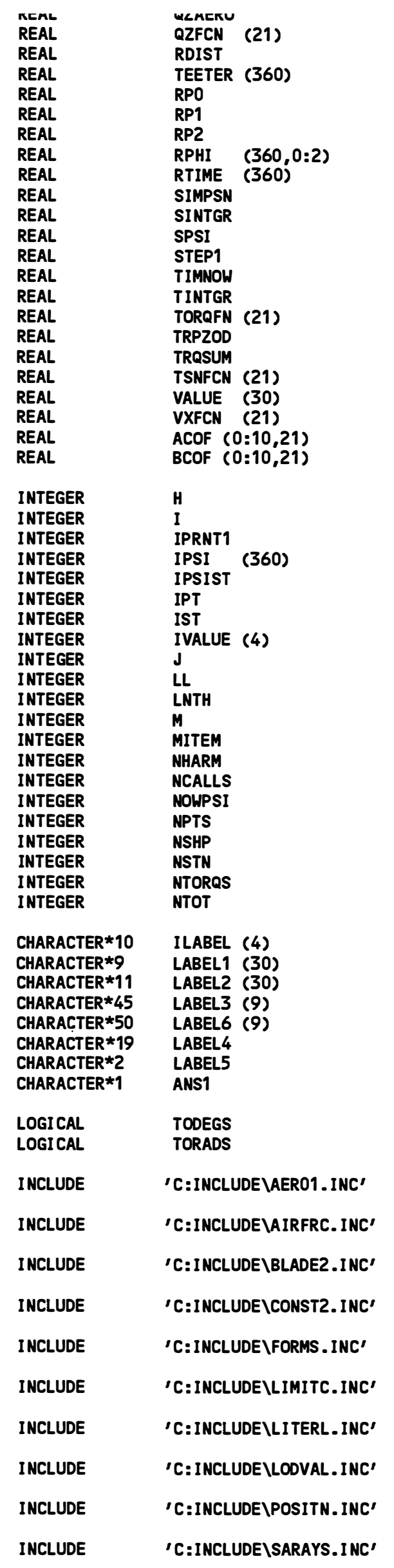




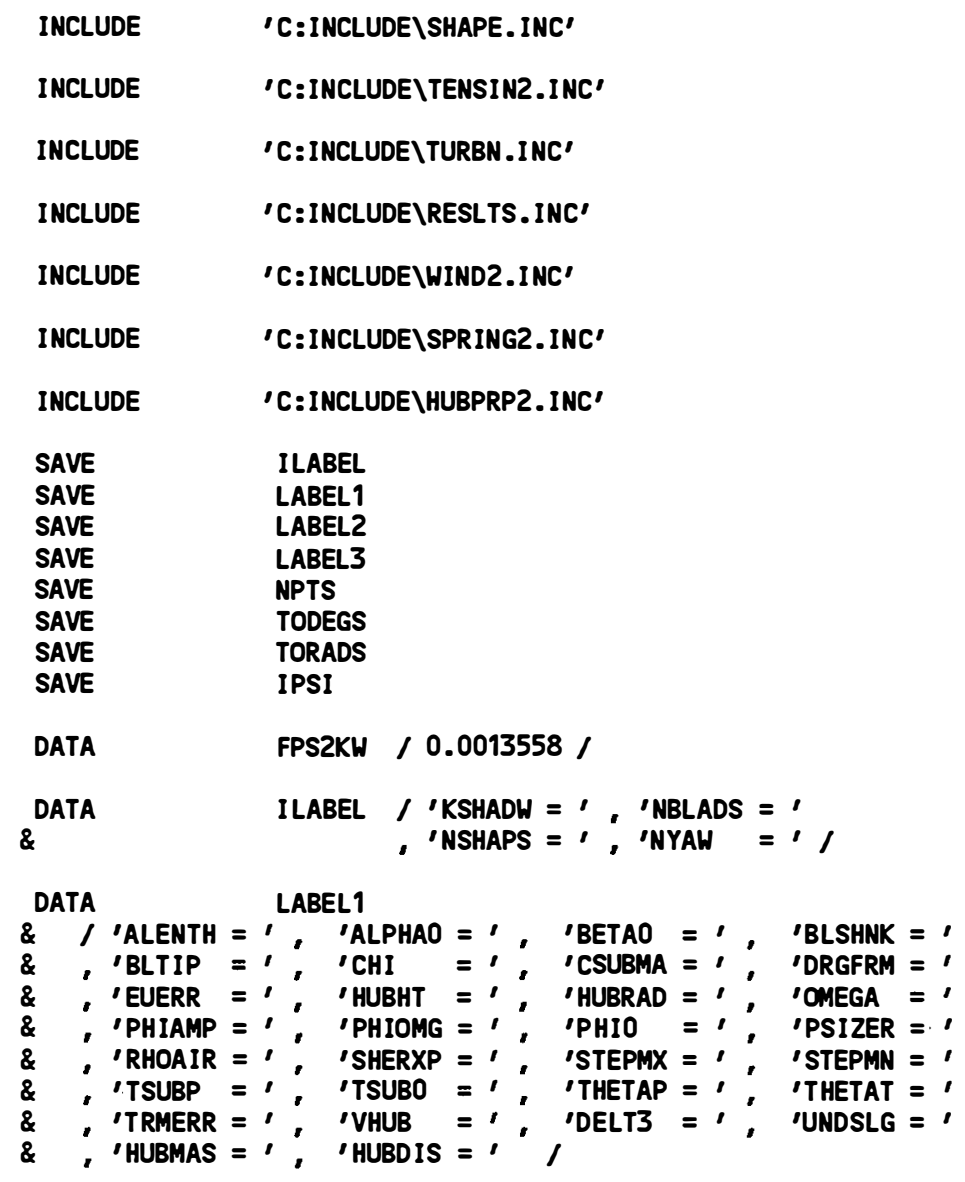

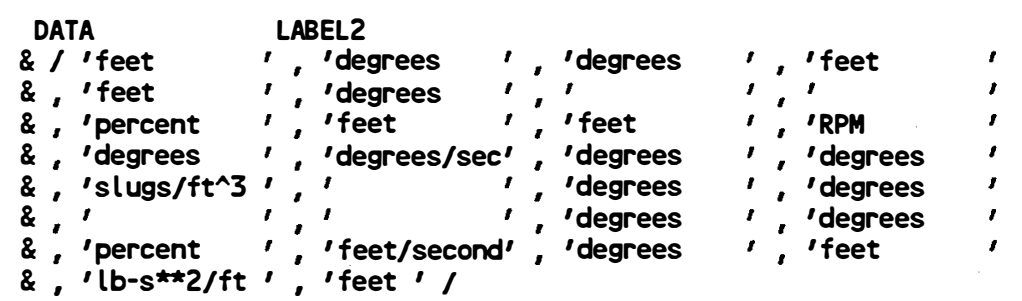

$\begin{array}{ll}\text { DATA } & \text { NPTS } / 21 / \\ \text { DATA } & \text { TODEGS / .TRUE. / } \\ \text { DATA } & \text { TORADS / . FALSE. / }\end{array}$

4000 FORMAT ( /// 37X , 'Analysis of Wind Turbine Blade Loads', 


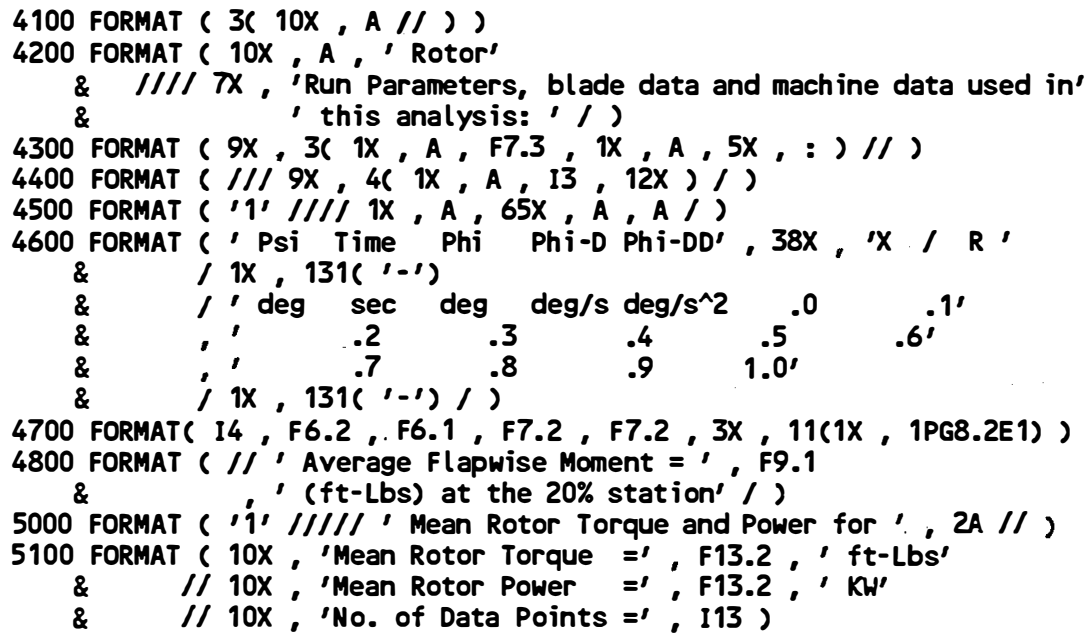

C Initialize torque count and sum.

NTORQS $=0$

TRQSUM $=0.0$

C Fill the IPSI array with PSI disk station values. This section C of code is executed only the first time this routine is called.

IF( NCALLS .EQ. 0 ) THEN

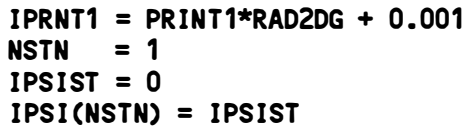


$\operatorname{RTIME}(1)=2 * P I *($ NCALLS - 1 )/OMEGA

DO 210 IST $=2$, NSTN

$210 \operatorname{RTIME}(I S T)=\operatorname{RTIME}(1)+\operatorname{IPSI}(I S T) /(\operatorname{RADZDG*OMEGA)}$

END IF

C

Initialize outer computation loop used for going around the disk.

PRODD $=0.5 * R H O A I R * C S U B M A$

DO 370 IST=1, NSTN

C

Compute the PHI values at this disk azimuth.

TIMNOW = RTIME(IST)

CALL NXTPHI (TIMNOW)

$\operatorname{RPHI}(I S T, 0)=\operatorname{PHI}(0)$

$\operatorname{RPHI}(I S T, 1)=\operatorname{PHI}(1)$

$\operatorname{RPHI}(I S T, 2)=\operatorname{PHI}(2)$

C

Compute constants used in computing the shear and moment

C and tension values.

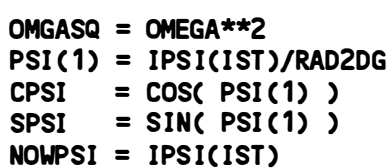

$\operatorname{TEETER}(I S T)=\operatorname{SNEW}(1,0, N O W P S I) /(B L T I P+H U B R A D)$

THETAC = THETAP + TEETER(IST)*SDELT3

CTHC $=$ COS(THETAC)

STHC = SIN(THETAC)

FACTR1 = OMGASQ *BETAO+

$2 *$ OMEGA*CPSI*PHI (1) + SPSI*PHI (2)

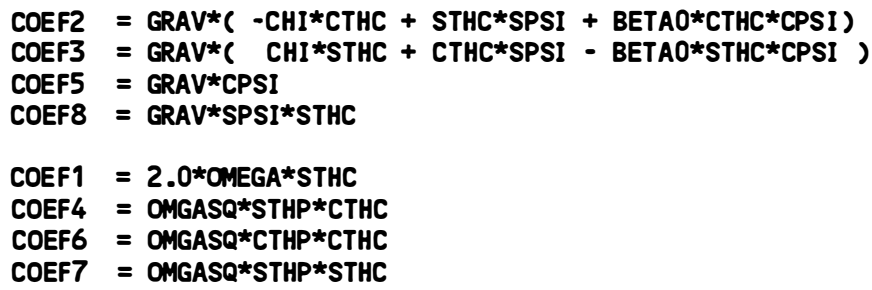

C

C

C

Fill the $V$ arrays.

$$
\begin{array}{ll}
v & =\text { RESLTS( }, 1) \\
\text { V-PRIME } & =\text { RESLTS( }, 2) \\
\text { V-DOT } & =\text { RESLTS( , ,3) }
\end{array}
$$

DO 305 IPT $=1$, NPTS

$V 1(0, I P T)=0$.

$\operatorname{V1}(1$, IPT $)=0$.

305 CONTINUE

DO 308 IPT $=1$, NPTS

$$
\begin{aligned}
& \text { \& } \\
& \text { SNEW(1, 0,NOWPSI )*CTHP } \\
& 308^{\&} \text { CONTINUE } \\
& \operatorname{SNEW}(1,1, \text { NOWPSI }) \star C T H P
\end{aligned}
$$$$
\text { V1 }(0, I P T)=V 1(0, I P T)+\operatorname{SHAPE}(1,0, I P T) *
$$$$
V 1(1, I P T)=V 1(1, I P T)+\operatorname{SHAPE}(1,0, I P T) *
$$ 
$\operatorname{RESLTS(IST,~IPT,2)~}=0.0$

RESLTS (IST,IPT,3) $=0.0$

DO 300 NSHP $=1$, NSHAPS

\&

\&

\&

\&

$\&$

\&

300

C

310

C

C

C

C
C
C
C
C
C

IF(NSHP .EQ. 1) THEN

RESLTS(IST, IPT , 1) = RESLTS(IST, IPT , 1)

+ SHAPE (NSHP, O, IPT) *SNEW(NSHP, 0, NOWPSI )*CTHP

RESLTS (IST, IPT, 2) = RESLTS( IST, IPT , 2)

+ SHAPE(NSHP, 1, IPT)*SNEW(NSHP, 0, NOWPSI )*CTHP

RESLTS (IST , IPT , 3) = RESLTS (IST, IPT, 3)

+ SHAPE(NSHP, 0,IPT)*SNEW(NSHP, 1, NOWPSI)*CTHP

ELSE

RESLTS(IST, IPT , 1) = RESLTS(IST, IPT , 1)

+ SHAPE (NSHP , 0, IPT)*SNEW(NSHP, 0 , NOWPSI )

RESLTS (IST , IPT , 2) = RESLTS(IST, IPT, 2)

+ SHAPE(NSHP, 1, IPT)*SNEW(NSHP, 0, NOWPSI)

RESLTS (IST , IPT , 3) = RESLTS(IST, IPT, 3)

+ SHAPE(NSHP, 0, IPT)*SNEW(NSHP, 1, NOWPSI)

ENDIF

CONTINUE

The values for $W(0, I P T)$ and $V V(1, I P T)$ are needed for the AERO and VREL routines.

$W(0, I P T)=$ RESLTS (IST, IPT, 1)

$W(1, I P T)=\operatorname{RESLTS}(I S T, I P T, 3)$

CONTINUE

Compute the aerodynamic force components and the QZ component form integrals and save them for use in computing the shears and moments.

Compute loads on the teeter damper

IF ( ABS(TEETER(IST)) . GE. BETA1) THEN

ELSE

DAMCOF = CDAMP

DAMCOF $=0$.

ENDIF

FDAMP(IST) $=$ DAMCOF $* \operatorname{SNEW(1,1,NOWPSI)/(HUBRAD+BLTIP)}$

BLDANG $=$ PSI $(1)$

CALL AERO ( NPTS , BLDANG )

This section of code computes the rotor torque component that produces the turbine shaft torque. The torque is averaged over the disk to give average rotor torque for one rotor revolution. This does not use weighted values. All values have equal weight regardless of the azimuth spacing. Thus these results will only be accurate when PRINTI and PRINT2 have the same value.

STEP1 $=$ BLTIP/( NPTS -1$)$

IF ( NOWPSI .LT. 360 ) THEN

DO $320 \mathrm{I}=1$, NPTS 
RDIST = HUBRAD + STEP $1 *(I-1)$

TORQFN(I $)=$ RDIST*( DAETA $(I) * C T H C+\operatorname{DAZETA}(I) * S T H C)$

CONTINUE

TRQSUM = TRQSUM + SIMPSN( 0, BLTIP , NPTS , TORQFN ) NTORQS $=$ NTORQS +1

END IF

DO 330 IPT=1, NPTS

$\begin{array}{ll}\text { DMSBAC } & =\text { PRODD*CHORD (IPT)*WH(IPT)**2 } \\ \text { QZFCN (IPT ) } & \text { DMSBAC + ESUBAC (IPT)*DAZETA (IPT) }\end{array}$

CONTINUE

DO 350 IPT=1, NPTS

PXAERO (IPT) $=$ TRPZOD ( IPT , BLTIP , DAETA , NPTS )

PYAERO(IPT) $=$ TRPZOD (IPT ', BLTIP ', DAZETA', NPTS )

C

Compute the constants needed to compute tension and shear.

PRDCTO $=0.0$

PRDCT1 $=0.0$

PRDCT2 $=0.0$

$\operatorname{PRODCT}(I P T)=0.0$

DO 340 NSHP=1, NSHAPS

IF( NSHP .EQ. 1) THEN

PRDCTO = PRDCTO + SNEW(NSHP, O, NOWPSI) *TCORLS(NSHP, IPT )*CTHP

PRDCT1 = PRDCT1 + SNEW(NSHP, 1, NOWPSI )*TCORLS (NSHP, IPT)*CTHP

PRDCT2 = PRDCT2 + SNEW(NSHP, 2, NOWPSI )*TCORLS(NSHP, IPT )*CTHP

PRODCT(IPT) $=$ PRODCT (IPT)+SNEW(NSHP, 0, NOWPSI $)$

ELSE

*CIFMOM(NSHP, IPT)*CTHP

PRDCTO = PRDCTO + SNEW(NSHP, O,NOWPSI )* TCORLS (NSHP, IPT)

PRDCT1 = PRDCT1 + SNEW(NSHP , 1 ;NOWPSI ) * TCORLS (NSHP, IPT)

PRDCT2 = PRDCT2 + SNEW(NSHP, 2, NOWPSI )* TCORLS (NSHP, IPT)

PRODCT (IPT) = PRODCT (IPT )+SNEW(NSHP, 0 , NOWPSI ) ENDIF

*CI FMOM(NSHP, IPT)

340 CONTINUE

\&

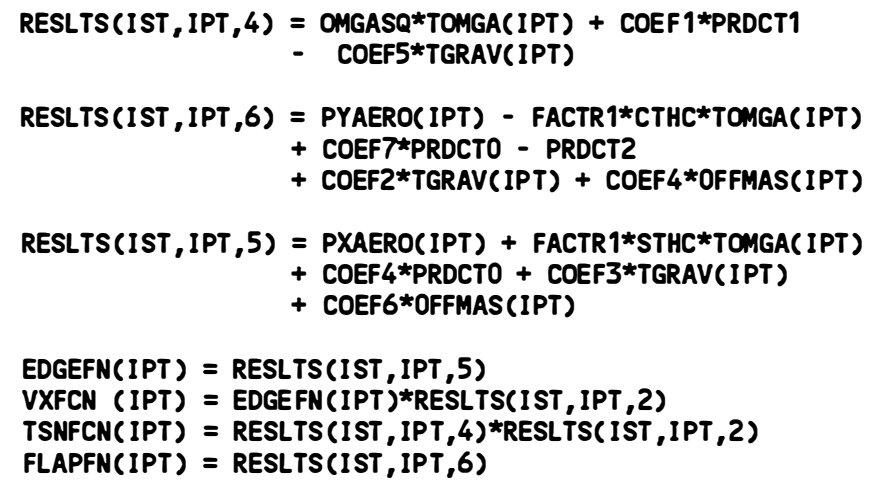
azimuth position. 


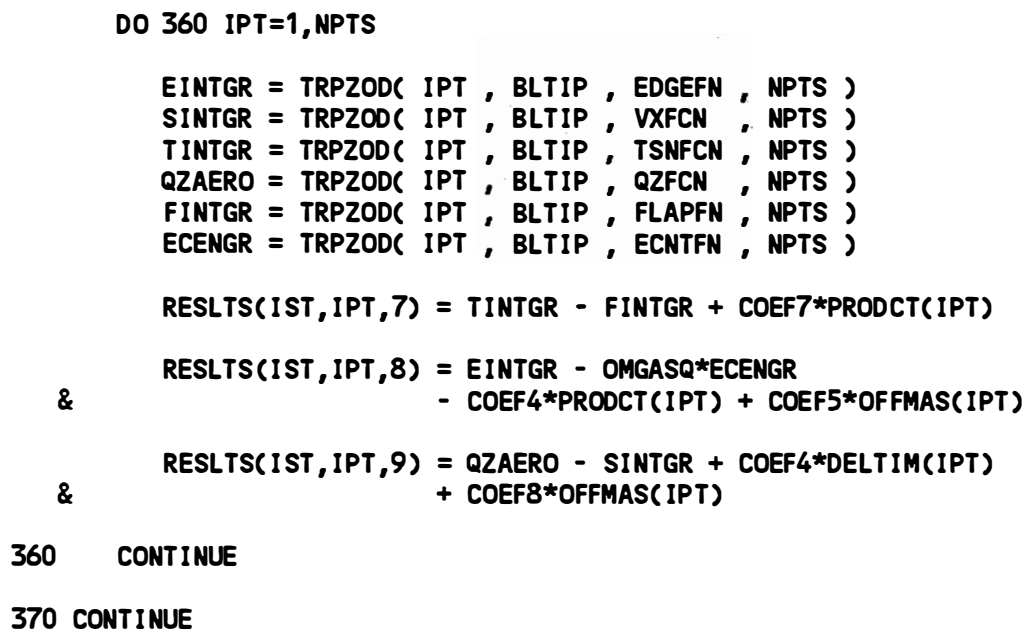

CALL CONVRT ( TODEGS )

IF ( MOD( NCALLS, NYAW+1 ) .EQ. 0 ) THEN

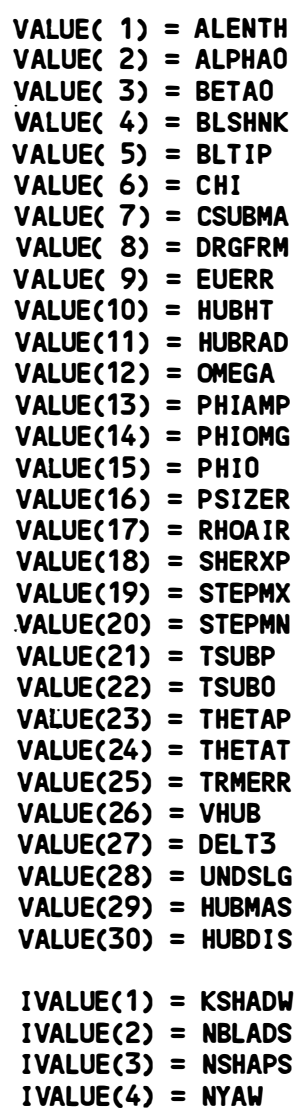


WRITE $(4,4400)$ ( ILABEL(I), IVALUe(I), I=1,4)

END IF

C Convert values back to radians for use in internal

C calculations.

CALL CONVRT ( TORADS )

C This section of code computes the mean flapwise moment at the

C $20 \%$ blade station for NPTS=21. If NPTS is changed, the second

C subscript of RESLTS( , ,7) must be changed also.

C

C

Programmer note:

Changed to what?

NTOT $=0$

AVEMOM $=0.0$

DO $410 L L=1, N S T N-1$

AVEMOM = AVEMOM + RESLTS(LL, 5,7)

NTOT $=$ NTOT +1

410 CONTINUE

AVEMOM = AVEMOM/NTOT

C Form the proper chart headings and print out the nine charts

C of the results from this run.

IF ( NCALLS .EQ. 0 ) THEN

LABEL4 = 'Trim Solution $\quad$,

LABEL5 $=$,

ELSE

LABEL4 = 'Yaw Solution, ReV \#'

WRITE (LABEL5,'(12.2)') NCALLS

END IF

$\operatorname{WRITE}(4, *), '$

WRITE $(4, *), \quad$, 'PSI = (DEG.), 'TEETER = (DEG.),

DO 450 II $=1$, NSTN

WRITE $(4, *)$ IPSI (II), TEETER (II)*RADZDG

450 CONTINUE

$\operatorname{WRITE}(4, *)$,

WRITE $(4, *)$ ' ', 'PSI $=($ DEG.)', 'Teeter Damper Loads $(f t-l b) '$

DO 460 II $=1$, NSTN

$\operatorname{WRITE}(4, *)$ IPSI (II), FDAMP(II)

460 CONTINUE

DO $430 M=1,9$

WRITE $(4,4500)$ LABEL3(M) , LABEL 4 , LABEL5

WRITE $(4,4600)$

DO $420 \mathrm{I}=1$,NSTN

$R P O=\operatorname{RPHI}(I, 0) * R A D 2 D G$

$R P 1=R P H I(I, 1) * R A D 2 D G$

$\operatorname{RP2}=\operatorname{RPHI}(1,2) * \operatorname{RAD2DG}$

\&

WRITE $(4,4700)$ IPSI(I) , RTIME(I) , RPO , RP1 , RP2

CONTINUE 
IF ( M.EQ. 7 ) WRITE $(4,4800)$ AVEMOM

430 CONTINUE

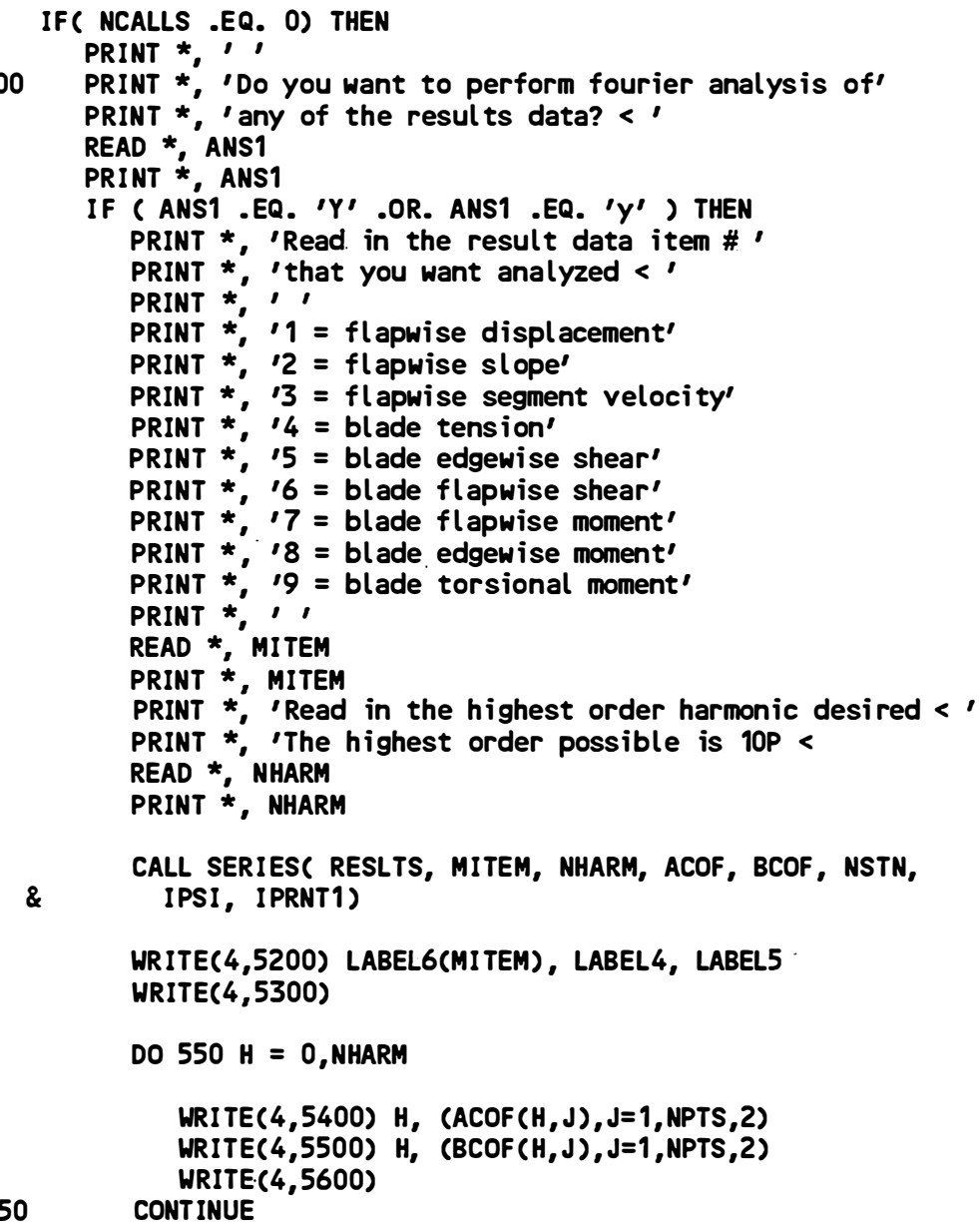

GO TO 500

ELSEIF ( ANS1 .NE. 'Y' .AND. ANS1 .NE. ' $Y$ ') THEN

ENDIF GO TO 600

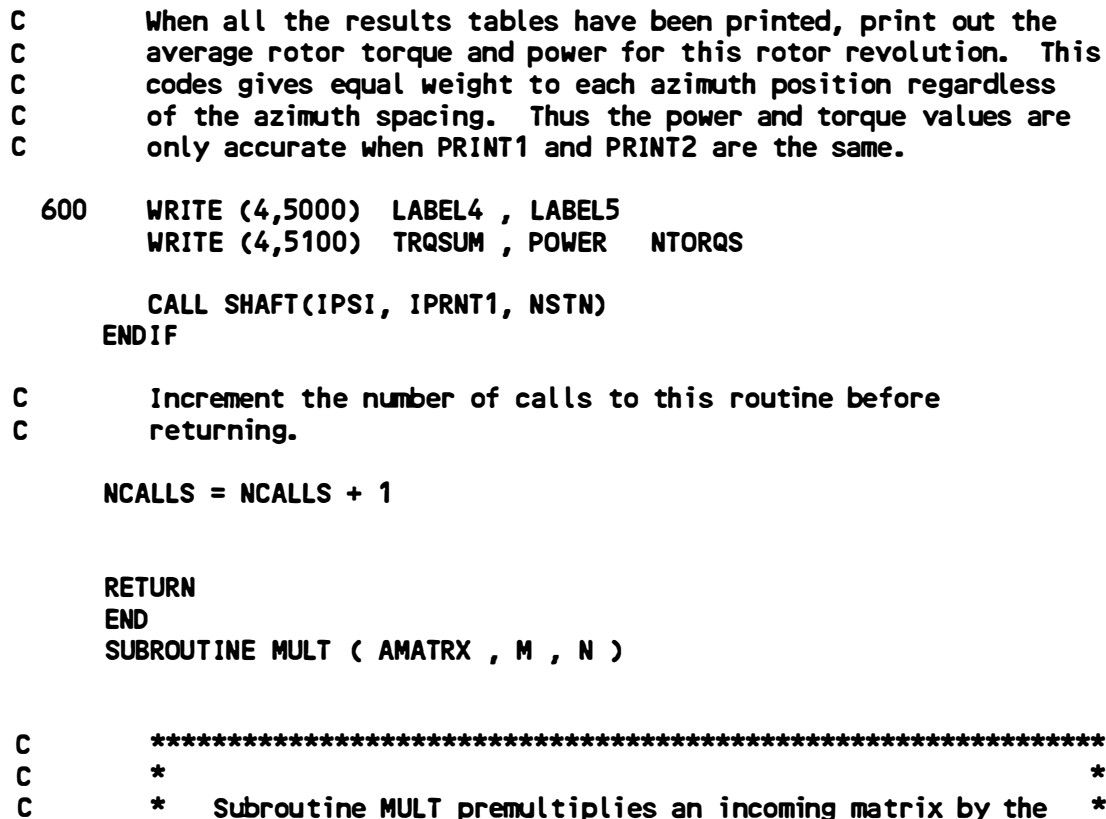




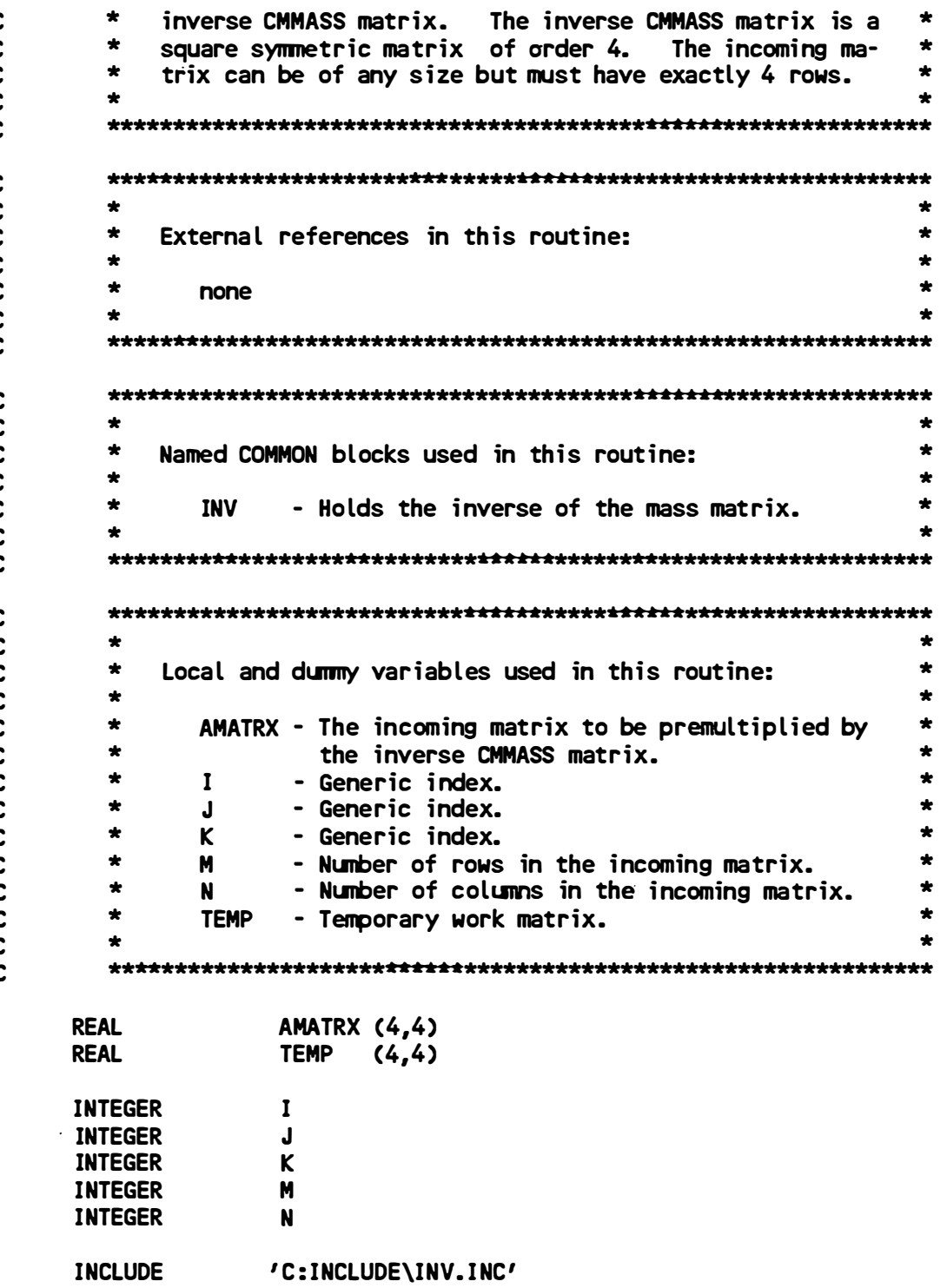

C Multiply AINVRS by AMATRX putting the result into TEMP.

DO $30 I=1, M$

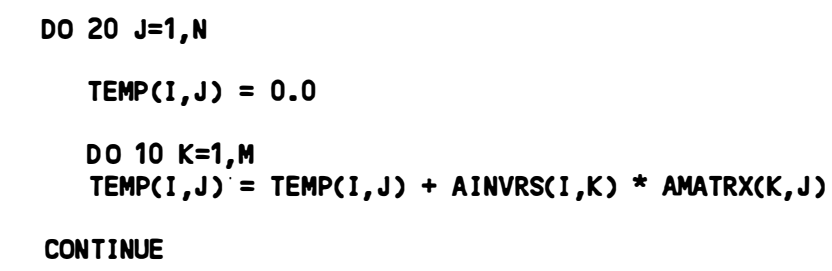

30 CONT INUE

C Move the resulting temporary matrix into the original

C incoming matrix.

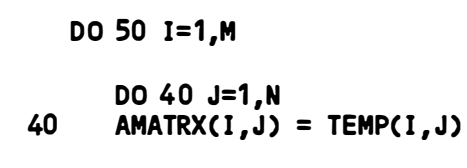

50 CONTINUE

RETURN 
* POSITN - Holds parameters related to blade position *

Local and dumiry variables used in this routine:

OMPHIT - Phase angle of the sinusoidal yaw function. It corresponds to the Omega-sub-Phi*T term of the formulation notes. The Omega-sub-Phi component is the yaw velocity amplitude divided by the yaw angle amplitude.

SOMPHT - Sine of Omega-sub-Phi*T.

TIMNOW - Current time. TIMNOW is identically zero for the trim solution computation.

$\begin{array}{ll}\text { REAL } & \text { OMPHIT } \\ \text { REAL } & \text { SOMPHT } \\ \text { REAL } & \text { TIMNOW } \\ \text { INCLUDE } & \text { 'C:INCLUDEIPOSITN.INC' } \\ \text { INCLUDE } & \text { 'C:INCLUDEITURBN.INC' }\end{array}$

IF ( TIMNOW .EQ. 0.0 ) THEN 
$\operatorname{PHI}(2)=0.0$

ELSE

Compute the yaw function and $i$ ts derivatives for times greater than time zero.

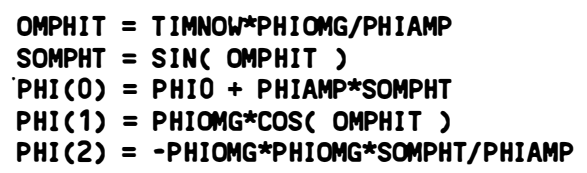

END IF

RETURN

END

SUBROUTINE NXTPSI ( IPSIST )

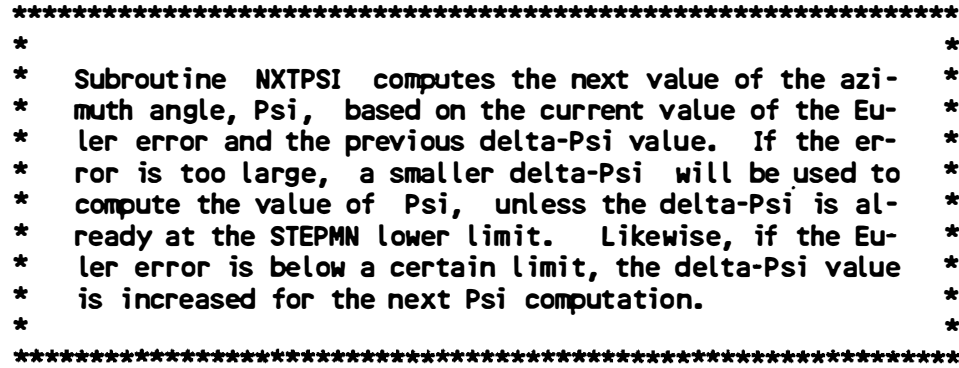

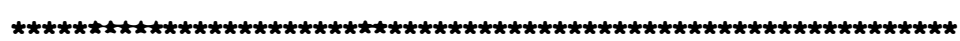

$\star$

* External references in this routine:

*

*

none

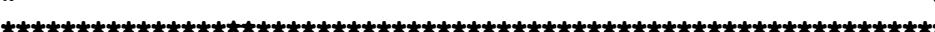

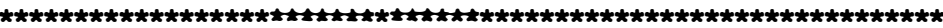

*

Named COMmON blocks used in this routine:

*

CONST - Turbine and other constants used in load *

* calculations.

* LIMITC - Holds values used in the LIMITS routine.

POSITN - Holds parameters related to blade position such as PHI, PSI, etc.

TURBN - Holds turbine parameters such as number of blades, rotor speed, etc.

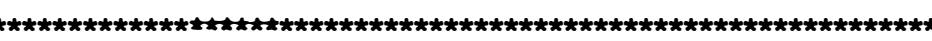

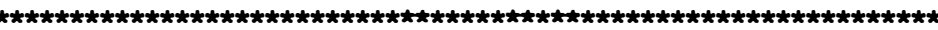

Local and dumy variables used in this routine:

IPSIST - The next STEPMX station in integer degrees.

NEW - Array index for new data.

OLD - Array index for old data.

PSIST - The next STEPMX station in radians.

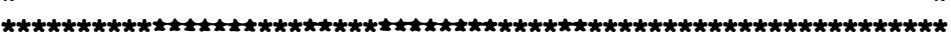

REAL

PSIST

INTEGER

INTEGER

INTEGER

IPSIST

NEW

OLD

INCLUDE

'C:INCLUDE ICONST2. INC'

INCLUDE

'C:INCLUDEXLIMITC.INC' 


$\begin{array}{ll}\text { INCLUDE } & \text { 'C:INCLUDE IPOSITN.INC' } \\ \text { INCLUDE } & \text { 'C:INCLUDE ITURBN.INC' } \\ \text { SAVE } & \text { NEW } \\ \text { SAVE } & \text { OLD } \\ & \\ \text { DATA } & \text { NEW } / 1 / \\ \text { DATA } & \text { OLD } / 0 /\end{array}$

C Check the error. If it is too large, decrease delta-Psi by

C $50 \%$. If it is very small, increase delta-Psi by $50 \%$. The

c value of delta-Psi must always be greater than STEPMN.

IF ( ERROR .GT. EUERR ) THEN

DELPSI (NEW) $=\operatorname{AMAX1}(0.5 *$ DELPSI (NEW), STEPMN )

ELSE IF ( ERROR -LT . 0.1*EUERR ) THEN

DELPSI (NEW) $=1.5 *$ DELPSI (NEW)

END IF

This section of code checks the position of the new Psi against the next STEPMX station. If the next Psi will go past the STEPMX station, the delta-Psi is adjusted to bring the next Psi directly onto the STEPMX point. If the next Psi will fall just short of a STEPMX station, the delta-Psi is adjusted to assure that it will not be necessary to use a delta-Psi smaller than STEPMN to reach the next STEPMX station on the next iteration.

\section{Programer note:}

The following logic can produce a delta-Psi that is less than STEPMN for two steps. It would then move back within tolerances. This case comes up when we are within slightly less than two STEPMNs of the next STEPMX station. This algorithm cannot produce a delta-Psi that is less than STEPMN/2.

MLB

PSIST $=$ IPSIST/RAD2DG

IF ( PSI (OLD)+DELPSI (NEW) .GT. PSIST ) THEN

DELPSI (NEW) = PSIST - PSI (OLD)

ELSE IF ( PSI(OLD)+DELPSI(NEW)+STEPMN .GT. PSIST ) THEN

IF ( (DELPSI (NEW) .NE. STEPMN) .OR. (ERROR .LE. EUERR) ) THEN DELPSI (NEW) $=0.5 *($ PSIST - PSI (OLD) )

END IF

END IF

Set new Psi and delta-Time.

PSI (NEW) = PSI (OLD) + DELPSI (NEW)

DELTAT(NEW) $=$ DELPSI (NEW)/OMEGA

C

Get time values if we're working on a yawing solution.

IF ( ITRIM .EQ. 0 ) $\operatorname{TIME}(N E W)=\operatorname{TIME(OLD)~+~DELTAT(NEW)~}$

RETURN

END

SUBROUTINE RUN ( NPTS , NEWSET , HAVRUN ) 

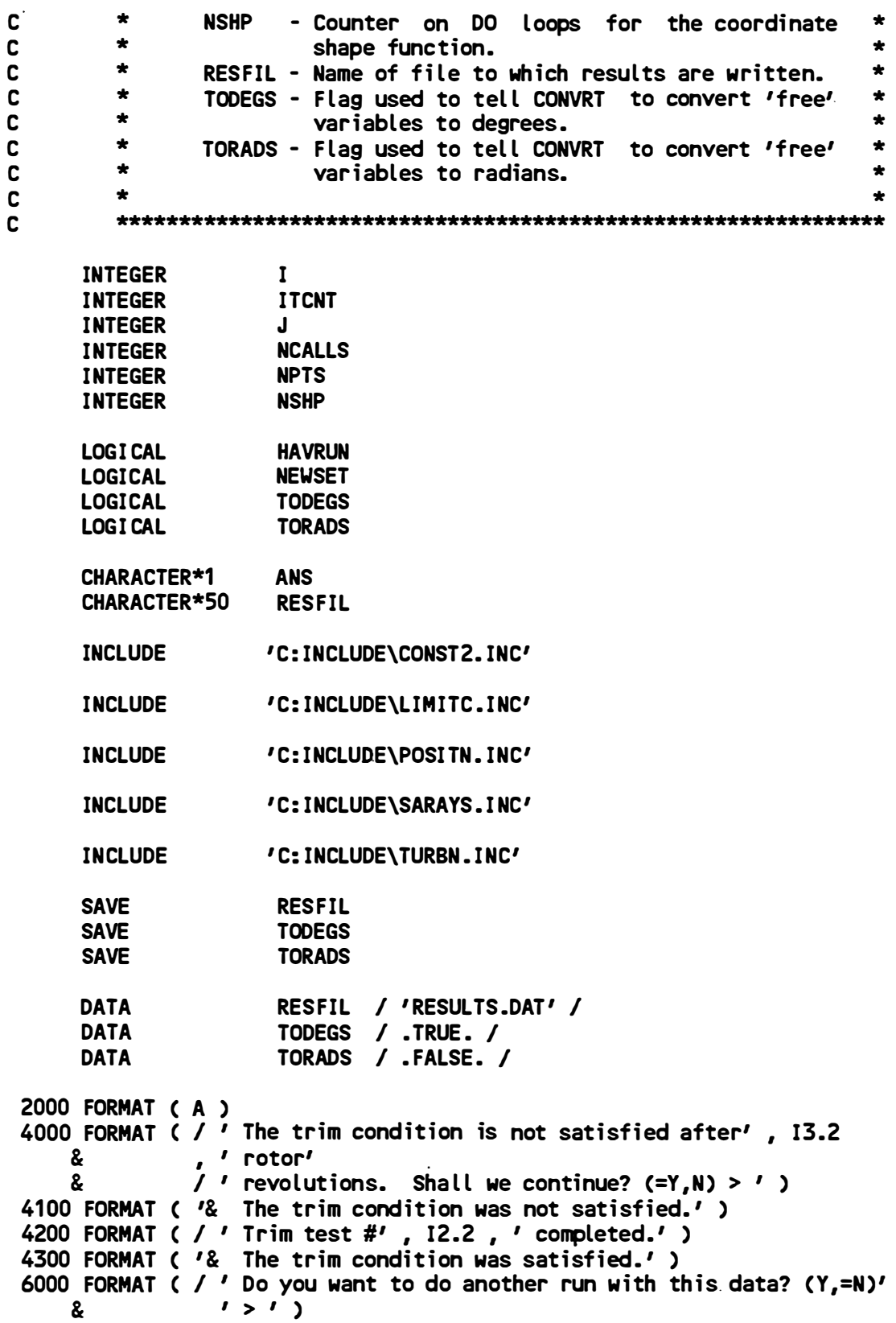

C The main run loop begins here. The interactive program seC quence starts.

100 CONTINUE

C

C Clear NCALLS which represents the number of calls to LODOUT.

C It is used to control printing of the title page. It is

c incremented in LODOUT just before returning.

NCALLS $=0$

C Set up a run by first calling the SETUP and LIMITS routines.

C Temporarily convert the units of the 'free' variables to de-

C grees and RPM for interactive use.

CALL CONVRT ( TODEGS )

CALL SETUP

CALL LIMITS

CALL CONVRT ( TORADS ) 
C Before starting the model run, check for potential error in the C yaw function. A value of zero for PHIAMP in a yaw solution

C will cause the program to abort.

IF ( ( NYAW .GT. 0 ) .AND. ( PHIAMP .EQ. 0.0 ) ) THEN

PRINT * ,

PRINT *', 'Error in input data. PHIAMP cannot be zero'

PRINT *, 'for a yaw solution. Please review the setup'

PRINT *, 'and limits values.'

GO TO 600

END IF

On the first pass through the RUN routine, invert the mass
C coefficient matrix and premultiply all the coefficient ma-

C trices by the result. The is done just once per data set.

IF ( NEWSET ) THEN

CALL SOLVE

NEWSET $=$.FALSE

END IF

200 PRINT *, ',

PRINT *, 'Are you ready to run the model? $(=\mathrm{Y}, \mathrm{N})>$

READ 2000, ANS

PRINT *, ANS

IF ( ( ANS .EQ. 'N') .OR. ( ANS .EQ. ' $n$ ' ) ) RETURN

IF ( ( ANS .NE. 'Y' ) .AND. ( ANS .NE. ' $Y$ ' )

\& .AND. ( ANS .NE. ' ', ) ) THEN

PRINT *, ' $\gg$ Invalid response. Please try again. $\ll<1$ GO TO 200

END IF

Open the file that is to contain the results.

CALL GETFIL ( RESFIL )

C

Zero the blade deflection, velocity and acceleration for the start of the trim solution run.

DO 320 NSHP $=1$, NSHAPS

DO $310 I=0,2$

DO $300 \mathrm{~J}=0,360$

SNEW(NSHP, I, J) $=0.0$

SOLD (NSHP, J) $=0.0$

300

310

CONTINUE

CONT INUE

CONT INUE

C Start the trim run. When the ITRIM flag is set to 2, the first pass through DSKREV will also execute the STRTUP startup routine. STRTUP computes the static deflection of the blade at the $\mathbf{2 7 0}$ degree azimuth position, under the given wind conditions. This static deflection is used to start the actual model run, and helps to assure rapid convergence to the trim solution by removing many of the startup transients associated with beginning execution without a realistic initial blade tip deflection.

ITRIM $=$

PSI (1) $=270 / R A D Z D G$

DELPSI (1) $=$ STEPMN

ERROR $=0.0$ 
CALL DSKREV ( NPTS )

ITRIM = 1 I Iterate for the trim solution. Keep count of the rotor re-
C volutions and give the user a chance to bail out every ten revolutions.

ITCNT $=0$

400 IF ( ITRIM .EQ. 1 ) THEN

IF ( ITCNT . NE. O ) THEN

IF ( MOD ( ITCNT, 10 ) .EQ. 0 ) THEN

PRINT 4000, ITCNT

READ 2000, ANS

PRINT *, ANS

IF ( ( ANS .EQ. 'N') .OR. ( ANS .EQ. 'n' ) ) THEN

PRINT *, , ,

PRINT *, 'STRAP terminated by user.'

PRINT *, ',

STOP

END IF

ELSE

PRINT 4100

END IF

END IF

C Initialize the azimuth angle and run another revolution.

Test the last set of solutions against this set for stabil-

ity. When TRMTST is satisfied, ITRIM will be set to zero

the loop will be terminated.

$\operatorname{PSI}(1)=0.0$

CALL DSKREV ( NPTS )

ITCNT $=$ ITCNT +1

CALL TRMTST ( ITRIM )

PRINT 4200, ITCNT

GO TO 400

END IF

C Trim solution completed.

PRINT 4300

C Calculate results of the trim solution.

CALL LODOUT ( NCALLS )

C Initialize the yaw run loop by starting time at zero. Run yaw

C solution NYAW times. Calculate the loads and print out the

C results after each disk revolution.

IF ( NYAW . GT. O ) THEN

$\operatorname{TIME}(1)=0.0$ 


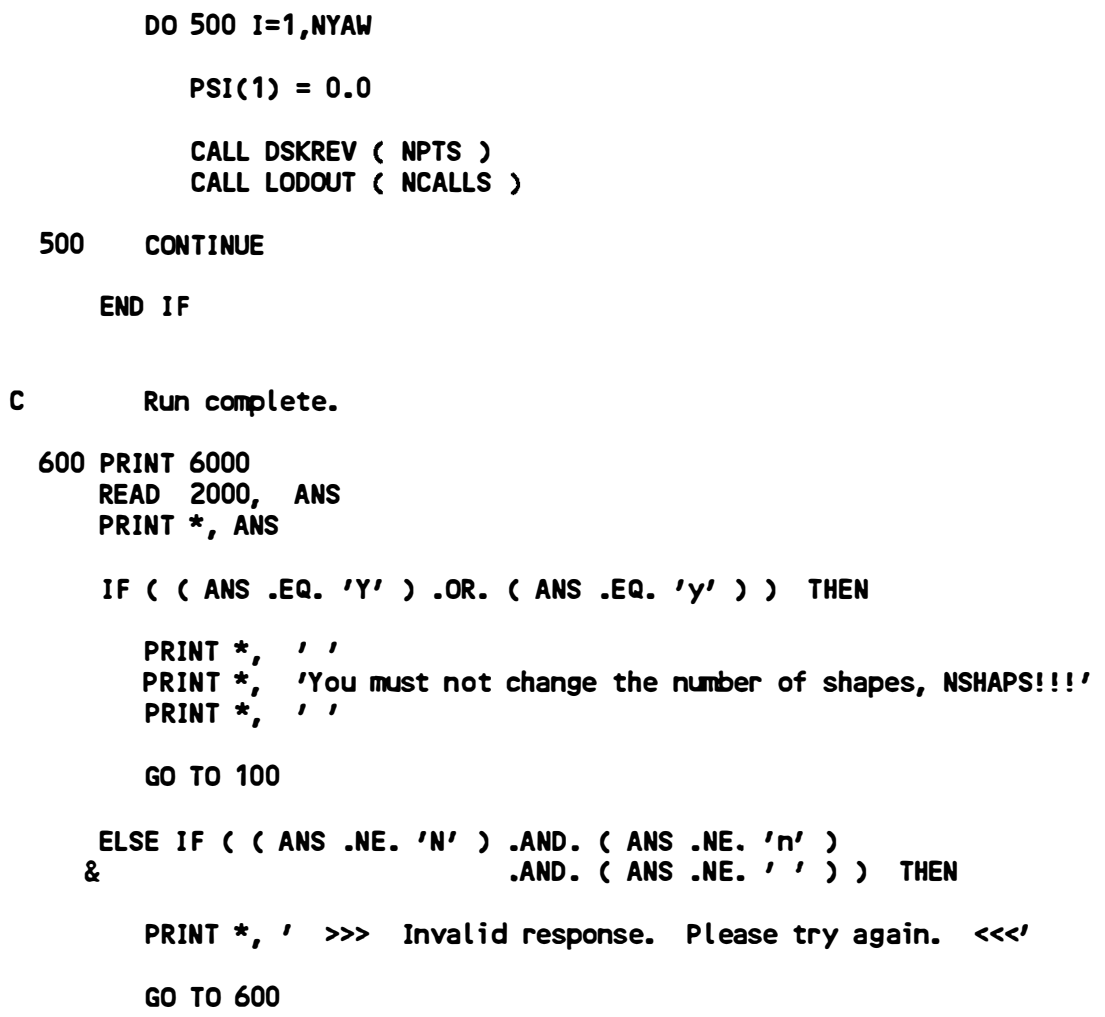


$\star$

* Local and dumiry variables used in this routine:

$\star$

* IPSIST - The next STEPMX station in integer degrees.

IPSIST - The next STEPMX station in

NSHAPS - Number of blade shape functions, 4 maximum.

NSHP - Counter on DO loops for the coordinate shape function.

STEMP - Temporary array holding the tip displacement values. The third dimension represents the order of the time derivative.

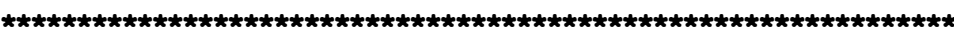

REAL

STEMP $(4,0: 1,0: 2)$

INTEGER IPSIST

INTEGER NEW

INTEGER NSHAPS

INTEGER NSHP

INCLUDE 'C:INCLUDEICONST2.INC'

INCLUDE 'C:INCLUDE ILIMITC.INC'

INCLUDE 'C:INCLUDE\POSITN.INC'

INCLUDE 'C:INCLUDEISARAYS.INC'

SAVE NEW

DATA NEW / $1 /$

Move the values stored in STEMP into SNEW.

DO 10 NSHP $=1$, NSHAPS

SNEW(NSHP, 0, IPSIST $)=$ STEMP (NSHP, NEW, 0)

SNEW(NSHP, 1, IPSIST) = STEMP (NSHP,NEW, 1)

SNEW(NSHP, 2, IPSIST) = STEMP(NSHP, NEW, 2)

10 CONTINUE

C Set the STEPMX station pointer, IPSIST, for the next STEPMX

C location on the rotor disk.

IPSIST $=$ IPSIST + STEPMX*RADZDG + 0.001

RETURN

END

SUBROUTINE SETUP

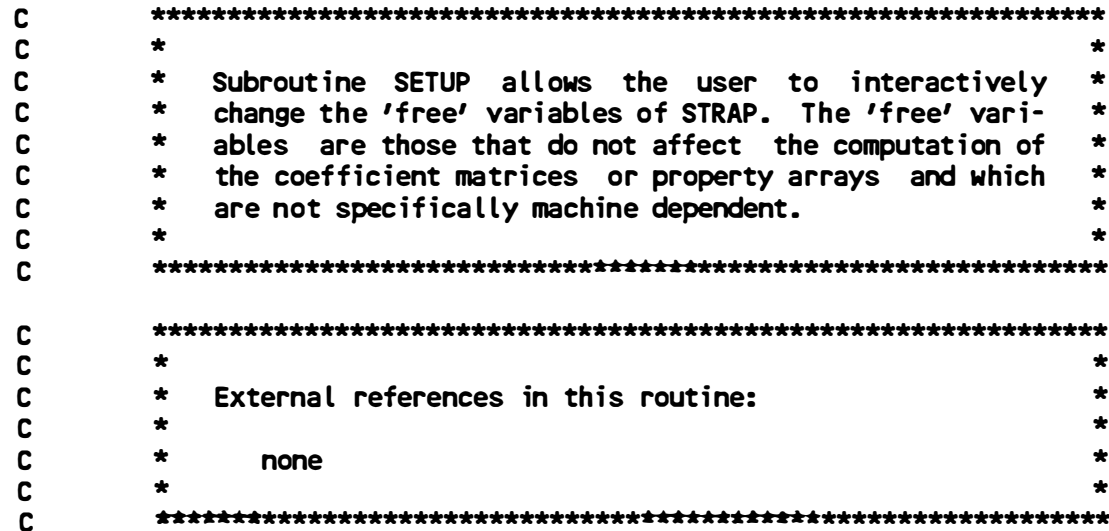




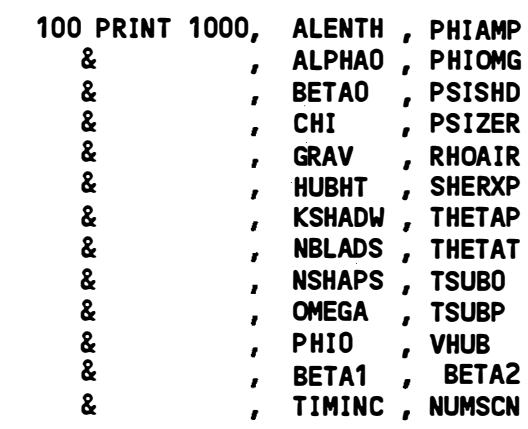

C Allow user to change values of the 'free' variables. Display. values of all variables after each change.

PRINT * , ',

200 PRINT * 'Would you like to change any values? $(Y,=N)>$ '

READ 2000, ANS

PRINT *, ANS

IF ( ( ANS .EQ. 'Y' ) .OR. ( ANS .EQ. ' $Y$ ' ) ) THEN

210 PRINT *, 'Enter the number of the variable you would Iike'

PRINT *, 'to change $(1-26)>$ '

READ *, ICASE

PRINT *, ICASE

GO TO $(300,302,305,310,315,320,325,330,335,340,345$

\& $\quad 350,355,360,365,370,375,380,385,390,392,395$

\& $\quad, 400,402,403,404)$ ICASE

PRINT *, , $\gg>$ Invalid response. Please try again. «<

PRINT *, , ,

GO TO 210

300 PRINT *, 'Enter new REAL value for ALENTH > '

READ *, ALENTH

PRINT *, ALENTH

GO TO 100

302 PRINT *, 'Enter new REAL value for ALPHAO > '

READ *, ALPHAO

PRINT *, ALPHAO

GO TO 100

305 PRINT *; 'Enter nEw REAL value for BETAO > '

READ *, BETAO

PRINT *, BETAO

GO TO 100

310 PRINT *, 'Enter new REAL value for $\mathrm{CHI}>$ '

READ * CHI

PRINT *, CHI

GO TO 100

315 PRINT *, 'Enter nEw REAL value for GRAV > '

READ *, GRAV

PRINT *, GRAV

GO TO 100

PRINT *, 'Enter new REAL value for HUBHT > '

READ * HUBHT

PRINT *, HUBHT

GO TO 100

PRINT *, 'Enter nEW INTEGER value for KSHADW > '

READ *, KSHADW

PRINT *, KSHADW

GO TO 100

PRINT *, 'Enter new INTEGER value for NBLADS > '

READ * NBLADS

PRINT *, NBLADS

GO TO 100 
335 PRINT *, 'Enter new INTEGER value for NSHAPS > '

READ *, NSHAPS

PRINT *, NSHAPS

GO TO 100

340 PRINT *, 'Enter new REAL value for OMEGA > '

READ *, OMEGA

PRINT *, OMEGA

GO TO 100

345 PRINT *, 'Enter new REAL value for PHIO > '

READ *, PHIO

PRINT * PHIO

GO TO 100

350 PRINT *, 'Enter new REAL value for PHIAMP > '

READ *, PHIAMP

PRINT *, PHIAMP

GO TO 100

355 PRINT *, 'Enter new REAL value for PHIOMG > ' READ *, PHIOMG

PRINT *, PHIOMG

GO TO 100

360 PRINT *, 'Enter new REAL value for PSISHD > '

READ *, PSISHD

PRINT *, PSISHD

GO TO 100

365 PRINT * 'Enter new REAL value for PSIZER > ' READ *, PSIZER

PRINT * PSIZER

GO TO 100

370 PRINT *, 'Enter nEw REAL value for RHOAIR > ' READ *, RHOAIR

PRINT *, RHOAIR

GO TO 100

375 PRINT *, 'Enter nEw REAL value for SHERXP > ' READ *, SHERXP

PRINT *, SHERXP

GO TO 100

380 PRINT *, 'Enter new REAL value for THETAP > '

READ * THETAP

PRINT *, THETAP

GO TO 100

385 PRINT *, 'Enter new REAL value for THETAT $>$ ' READ *, THETAT

PRINT *, THETAT

GO TO 100

390 PRINT *, 'Enter new REAL value for TSUBO > ' READ *, TSUBO

PRINT * TSUBO

GO TO 100

392 PRINT *, 'Enter new REAL value for TSUBP > ' READ * TSUBP

PRINT *, TSUBP

GO TO 100

395 PRINT *, 'Enter new REAL value for VHUB > '

READ * VHUB

PRINT *, VHUB

GO TO 100

400 PRINT *, ' Enter new REAL value for BETA1>'

READ *, BETA1

PRINT * BETA1

GO TO 100

402 PRINT *, ' Enter new REAL value for BETA2>'

READ *, BETA2 
PRINT *, BETA2

GO TO 100

403

PRINT *, ' Enter new REAL value for TIMINC>'

READ *, TIMINC

PRINT *, TIMINC

GO TO 100

404

PRINT *, ' Enter

READ *, NUMSCN

PRINT *, NUMSCN

GO TO 100

ELSE IF ( ( ANS .NE. 'N') .AND. ( ANS .NE. ' $n$ ')

.AND. ( ANS .NE.', ) ) THEN

PRINT *, , $\gg>$ Invalid response. Please try again. «<!

PRINT *, 1,

GO TO 200

END IF

Compute commonly used constants.

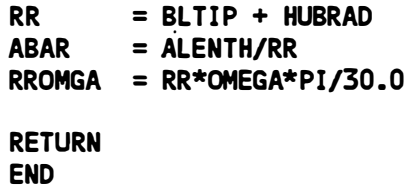

FUNCTION SIMPSN ( LOWLIM , UPLIM , NPTS , FOFX )

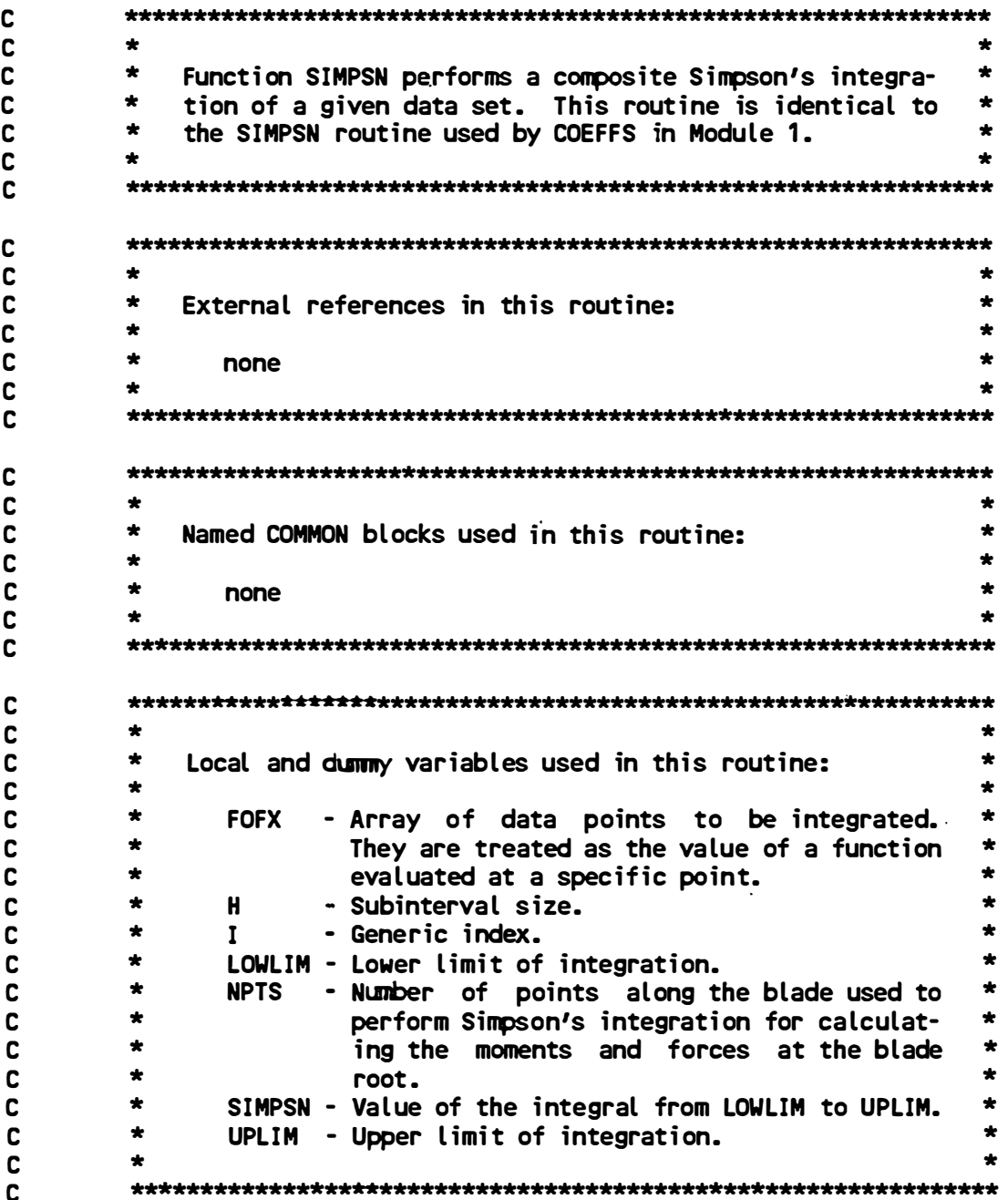

D-74 


$\begin{array}{ll}\text { REAL } & \text { FOFX (21) } \\ \text { REAL } & \text { H } \\ \text { REAL } & \text { LOWLIM } \\ \text { REAL } & \text { SIMPSN } \\ \text { REAL } & \text { UPLIM } \\ & \\ \text { INTEGER } & \text { I } \\ \text { INTEGER } & \text { NPTS }\end{array}$

C

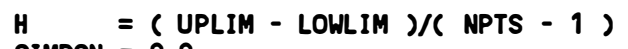

H $\quad=($ UPLIM - LOWLIM )/( NPTS - 1$)$

Compute the subinterval size and initialize the integral.

SIMPSN $=0.0$

Add in the intermediate points. In the formulation, all even numbered points have coefficient of 4 . In this case, the index must be shifted to form the proper coefficient.

DO $10 \quad I=2$, NPTS $-1,2$

10 SIMPSN $=$ SIMPSN $+4.0 *$ FOFX $(I)+2.0 *$ FOFX $(I+1)$

SIMPSN $=$ SIMPSN + FOFX(1) - FOFX(NPTS)

SIMPSN $=H *$ SIMPSN $/ 3.0$

RETURN

END

SUBROUTINE SOLVE 


$$
\text { REAL }
$$

TEMP $(4,4)$

INTEGER

INTEGER

INTEGER

I

INCLUDE

'C:INCLUDE INV. INC'

INCLUDE

'C:INCLUDEYMATRX1.INC'

INCLUDE

'C: INCLUDE VMATRX2.INC'

INCLUDE

'C:INCLUDE TTURBN.INC'

INCLUDE

'C:INCLUDEISPRING2.INC'

4000 FORMAT $(1 \mathrm{X}, 4 \mathrm{~F} 12.7 /)$

C

Invert the CMMASS matrix.

CALL INVERT ( CMMASS , AINVRS , NSHAPS )

C Premultiply the other coefficient matrices by the inverse

C CMMASS matrix.

CALL MULT ( CKBEND, NSHAPS, NSHAPS )

CALL MULT ( CKTOMG, NSHAPS, NSHAPS )

CALL MULT ( CKTGRV, NSHAPS, NSHAPS )

CALL MULT ( CKQLOD , NSHAPS , NSHAPS )

C The coefficient matrix CKTCRL must be separated into individual C 2-dimensional matrices for multiplication by the inverse CMMASS C matrix. Then the individual matrices are recombined into the C original 3-dimensional form.

DO $140 \mathrm{I}=1$, NSHAPS

DO $110 \mathrm{~J}=1$, NSHAPS

DO $100 \mathrm{~K}=\mathrm{J}$, NSHAPS

$\operatorname{TEMP}(J, K)=\operatorname{CKTCRL}(I, J, K)$

$\operatorname{TEMP}(K, J)=\operatorname{CKTCRL}(I, K, J)$

100

CONT INUE

110

CONTINUE

CALL MULT ( TEMP , NSHAPS , NSHAPS )

DO $130 \mathrm{~J}=1$, NSHAPS

DO $120 \mathrm{~K}=\mathrm{J}$, NSHAPS

$$
\begin{aligned}
& \operatorname{CKTCRL}(I, J, K)=\operatorname{TEMP}(J, K) \\
& \operatorname{CKTCRL}(I, K, J)=\operatorname{TEMP}(K, J)
\end{aligned}
$$

120

CONTINUE

130

CONTINUE

140 CONTINUE

C Load three of the 1-dimensional matrices into a single matrix

C

C
of order 4 by 3 and premultiply it by the inverse CMMASS ma- trix. Then move the resulting colums back into the original vectors.

DO $200 \mathrm{~J}=1$, NSHAPS

$\operatorname{TEMP}(J, 1)=\operatorname{CMRIGD}(J)$ 
$\operatorname{TEMP}(\mathrm{J}, 2)=\operatorname{CMBLNC}(\mathrm{J})$

$\operatorname{TEMP}(J, 3)=\operatorname{CMGRAV}(J)$

200 CONTINUE

CALL MULT ( TEMP , NSHAPS , 3 )

DO $210 \mathrm{~J}=1$,NSHAPS

$\operatorname{CMRIGD}(J)=\operatorname{TEMP}(J, 1)$

$\operatorname{CMBLNC}(J)=\operatorname{TEMP}(J, 2)$

$\operatorname{CMGRAV}(J)=\operatorname{TEMP}(J, 3)$

210 CONTINUE

C

Repeat the same process for the special teetering vectors.

DO $300 \mathrm{~J}=1$, NSHAPS

$\operatorname{TEMP}(J, 1)=\operatorname{CMRGD} 1(\mathrm{~J})$

$\operatorname{TEMP}(J, 2)=\operatorname{CMGRV1}(\mathrm{J})$

$\operatorname{TEMP}(\mathrm{J}, 3)=\operatorname{CMHUB} 1(\mathrm{~J})$

300 CONTINUE

CALL MULT ( TEMP , NSHAPS , 3 )

DO $310 \mathrm{j}=1$, NSHAPS

$\operatorname{CMRGD} 1(J)=\operatorname{TEMP}(J, 1)$

$\operatorname{CMGRV1}(\mathrm{J})=\operatorname{TEMP}(\mathrm{J}, 2)$

$\operatorname{CMHUB} 1(J)=\operatorname{TEMP}(J, 3)$

310 CONTINUE

DO $330 \mathrm{~J}=1$, NSHAPS

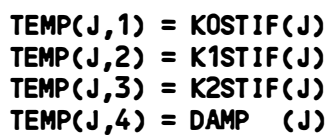

330 CONTINUE

CALL MULT( TEMP, NSHAPS, 4)

DO $340 \mathrm{~J}=1$, NSHAPS

$$
\begin{aligned}
& \operatorname{KOSTIF}(J)=\operatorname{TEMP}(J, 1) \\
& \operatorname{K1STIF}(J)=\operatorname{TEMP}(J, 2) \\
& \operatorname{K2STIF}(J)=\operatorname{TEMP}(J, 3) \\
& \operatorname{DAMP}(J)=\operatorname{TEMP}(J, 4)
\end{aligned}
$$

340 CONTINUE

C Premultiply the CMmASS matrix by $i$ ts inverse and save for diag-

C nostic purposes. Print out the result.

CALL MULT ( CMMASS , NSHAPS , NSHAPS )

PRINT *, ,

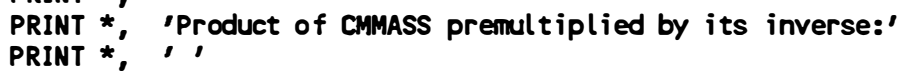

DO $400 \mathrm{I}=1$, NSHAPS

400 PRINT 4000, ( CMMASS $(I, J), J=1$, NSHAPS )

C CMMASS is replaced by its inverse for use in the RUN routine

C in computing the solution for the aerodynamic forces.

DO $500 \mathrm{I}=1$, NSHAPS

DO $510 \mathrm{~J}=1$, NSHAPS 
AIRFRC - Holds values used in aerodynamic calculations.

CONST - Turbine and other constants used in load calculations.

MATRX1 - Holds stiffness coefficient matrices.

MATRX2 - Holds other matrices related to coriolis stiffening, gravity, etc.

POSITN - Holds parameters related to blade position such as PHI, PSI, etc.

SARAYS Holds new and old values for the general:ized coordinates.

START Holds initial blade deflection.

TURBN. - Holds turbine parameters such as number of $\star$ blades, rotor speed, etc.

BLDANG - Blade azimuth position used in the teetering rotor option.

FAER01 - Aerodynamic force on blade \#1.

FAER02 - Aerodynamic force on blade $\# 2$.

FSTAR - Complicated term.

I - Generic index.

IDENT - The identity matrix.

$K$ - Generic index.

KINVRS - Inverse of KSTAR matrix.

KSTAR - Complicated term.

$L \quad$ Generic index.

M - Generic index.

NPTS - Number of points along the blade used to perform Simpson's integration for calculating the moments and forces at the blade root. (passed from STRAP1)

OMGSQR - Omega^2.

STEMP - Temporary array holding the tip displace- 


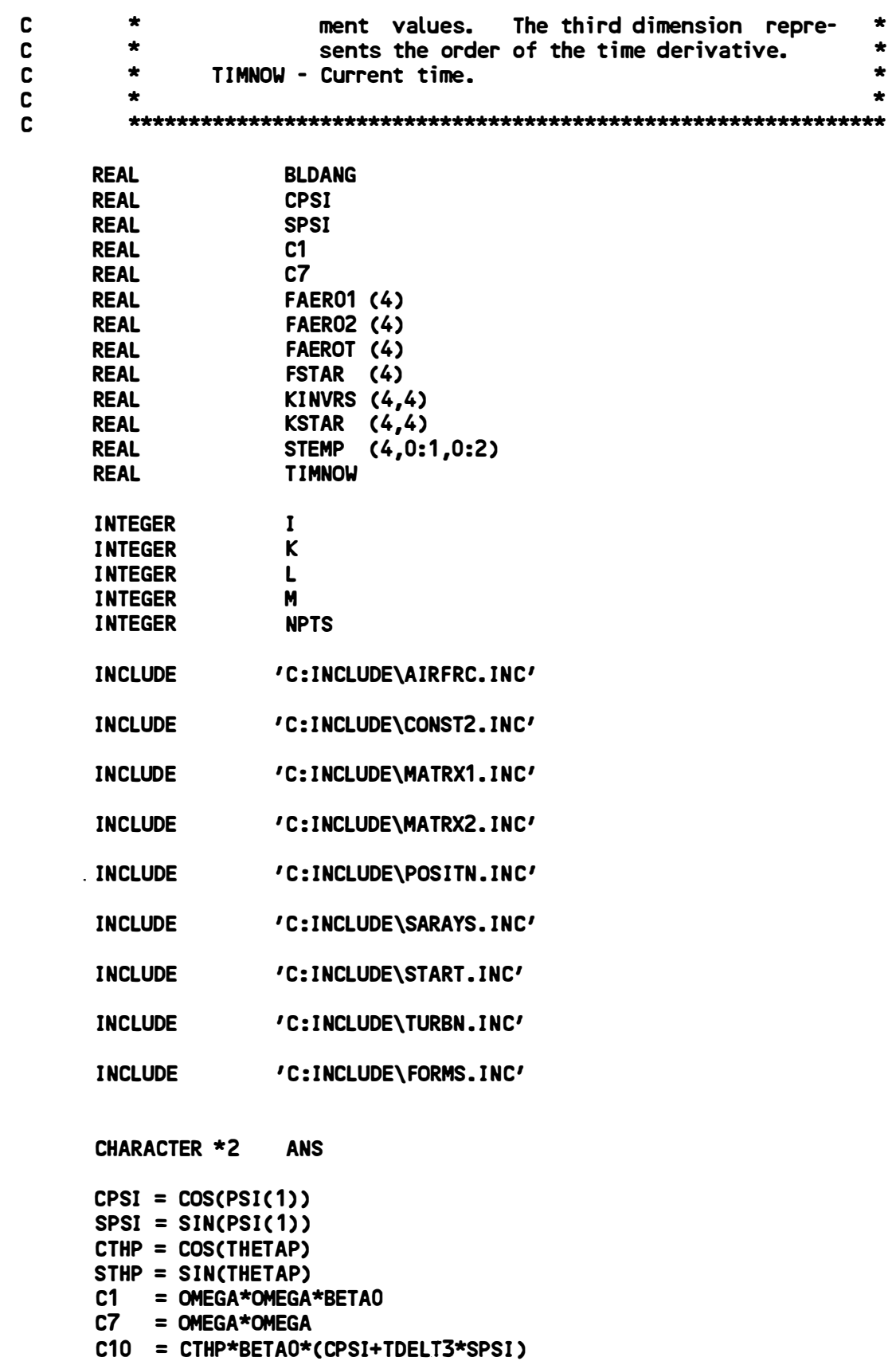

PRINT *, ,

PRINT *, ' Do you want to input your own values for the'

PRINT *, ' initial modal deflections, or do you want'

PRINT *,' SUBROUTINE STRTUP to calculate values for you?'

PRINT *,

PRINT *, " If you want to input your own then input a "Y"'

PRINT *, ' or a "y", If you want SUB STRTUP to do it'

PRINT *, , input an' "N" or an "n" $>$ ",

READ *, ANS

PRINT *, ANS

IF( ANS .EQ. 'N' .OR. ANS .EQ. ' $n$ ') THEN

Initialize some arrays.

DO $120 \mathrm{M}=1$, NSHAPS

DO $100 \mathrm{I}=0,2$

$\operatorname{STEMP}(M, 0, I)=0.0$ 
$\operatorname{STEMP}(M, 1, I)=0.0$

100

CONTINUE

120 CONTINUE

C

C

Compute the FAERO values for use in calculating the FSTAR values. These are actually FAERO multiplied by the inverse CMMASS matrix.

TIMNOW $=\operatorname{TIME}(1)$

CALL NXTPHI ( TIMNOW )

BLDANG $=\operatorname{PSI}(1)+P I$

CALL FORM1 ( STEMP , NPTS , NSHAPS , BLDANG )

THETAC $=$ THETAP + BETA*SDELT3

STHP = SIN(THETAC)

CTHP $=$ COS(THETAC)

TTHP = TAN (THETAC)

CALL AFORCE ( NPTS BLDANG)

200

DO $200 M=1$, NSHAPS

$F A E R 02(M)=F A E R O(M)$

BLDANG $=$ PSI $(1)$

CALL FORMI ( STEMP , NPTS , NSHAPS , BLDANG )

THETAC = THETAP + BETA*SDELT3

STHP = SIN(THETAC)

CTHP = COS (THETAC)

TTHP = TAN(THETAC)

CALL AFORCE ( NPTS, BLDANG)

DO $210 \mathrm{M}=1$, NSHAPS

210 FAERO1 $(M)=$ FAERO(M)

DO $220 M=1$, NSHAPS

220 FAEROT $(M)=0.5 *($ FAER01 $(M)+\operatorname{FAER02}(M) *(-1) * * M)$

C

Compute the FSTAR values.

C

$\operatorname{FSTAR}(1)=$ GRAV*C10*CMGRV1(1)

+ FAEROT( 1 )

DO $300 \mathrm{M}=2$, NSHAPS

$\&$

$\operatorname{FSTAR}(M)=$ FAEROT(M)

300 CONTINUE

C

Compute the KSTAR values.

DO $410 \mathrm{M}=1$, NSHAPS

DO $400 K=1$, NSHAPS

$400 \operatorname{KSTAR}(M, K)=\operatorname{CKBEND}(M, K)+\operatorname{C7}$ CKTOMG $(M, K)$

410 CONTINUE

C

Invert the KSTAR matrix.

CALL INVERT ( KSTAR , KINVRS , NSHAPS ) 
DO 510 L=1, NSHAPS

$$
S O(L)=0.0
$$

DO $500 \mathrm{M}=1$, NSHAPS

$500 S O(L)=S O(L)+\operatorname{KINVRS}(L, M) *$ FSTAR $(M)$

510 CONTINUE

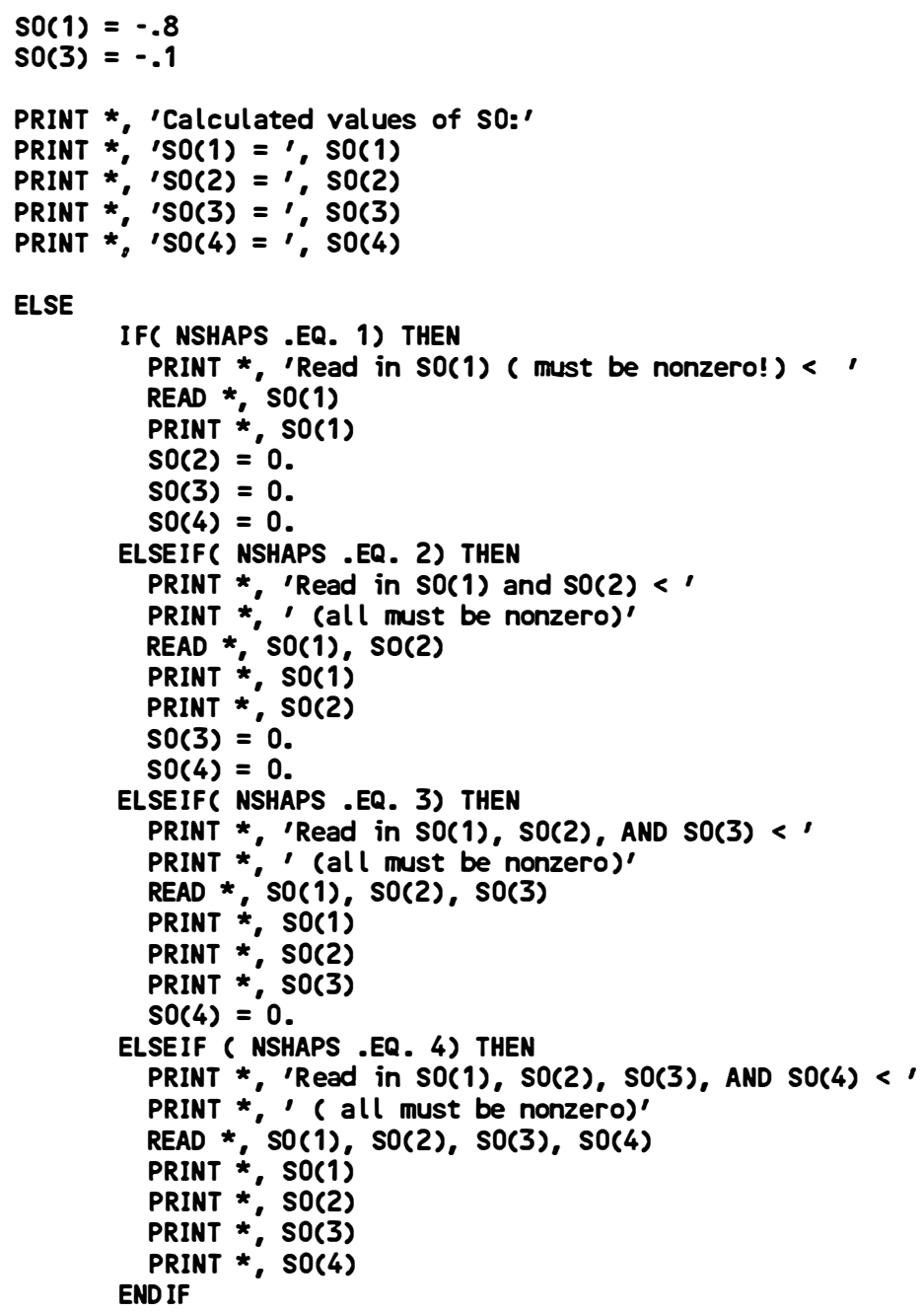

ENDIF

610 CONTINUE

RETURN

END

SUBROUTINE TRACE ( STEMP , NPTS ) 


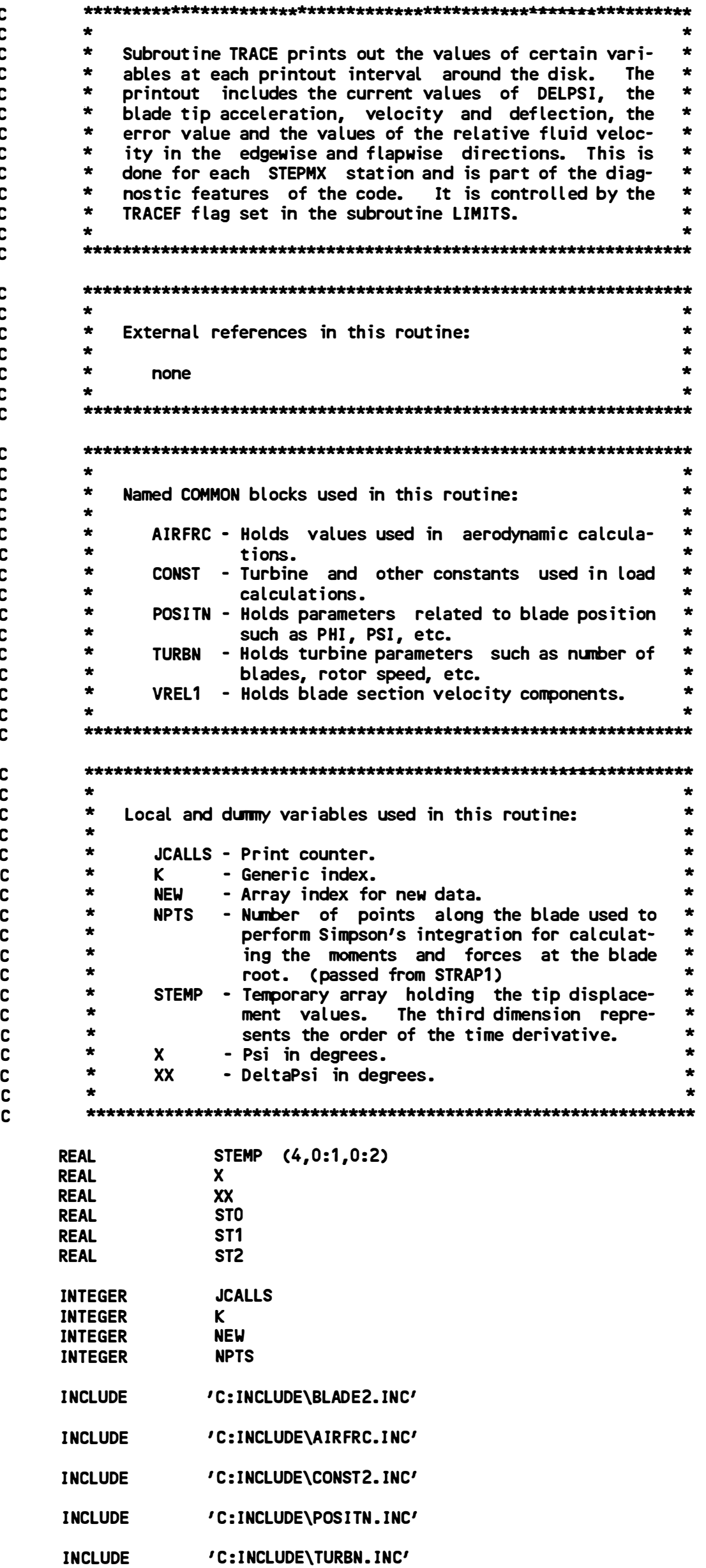




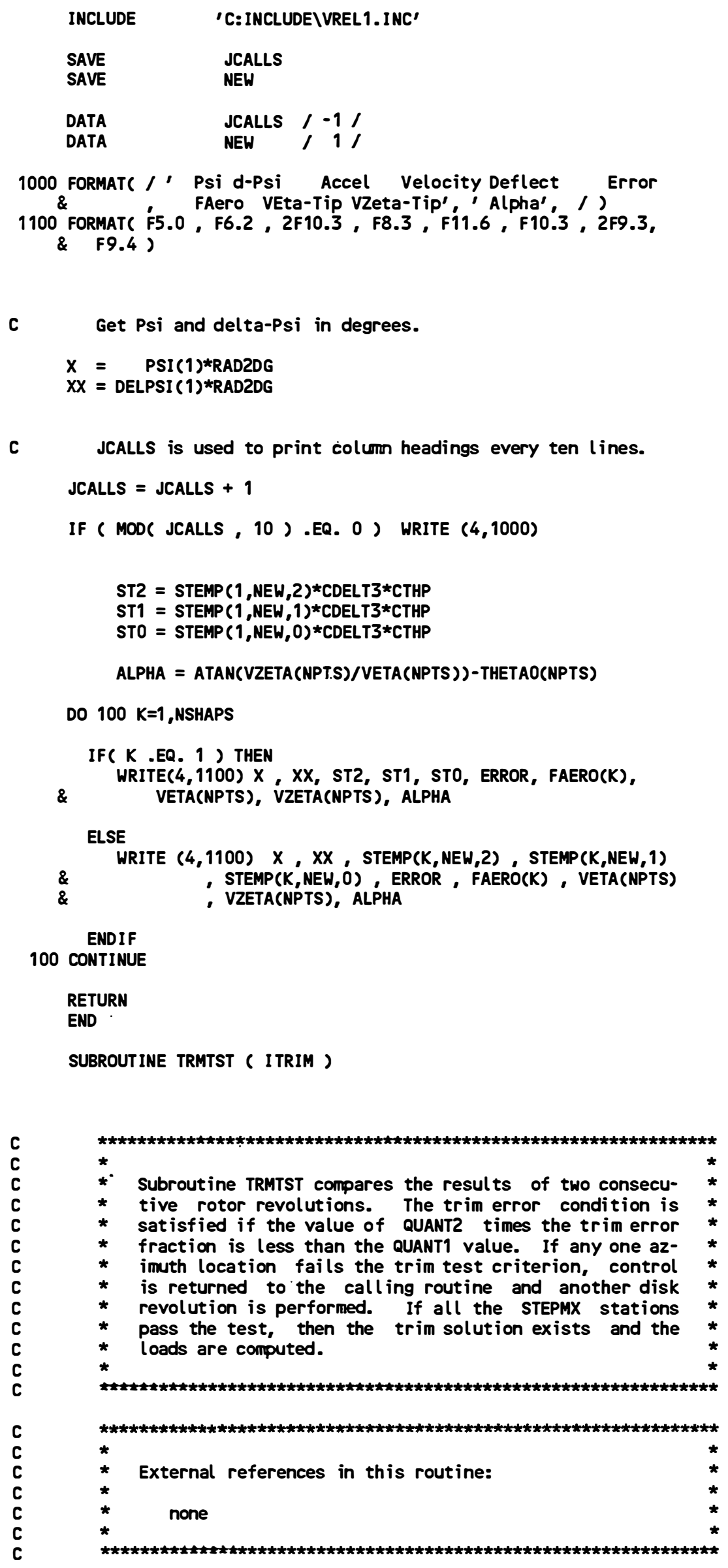


Named COMMON blocks used in this routine:

CONST - Turbine and other constants used in load * calculations.

LIMITC - Holds values used in the LIMITS routine.

SARAYS - Holds new and old values for the generalized coordinates.

START - Holds initial blade deflection.

TURBN - Holds turbine parameters such as number of blades, rotor speed, etc.

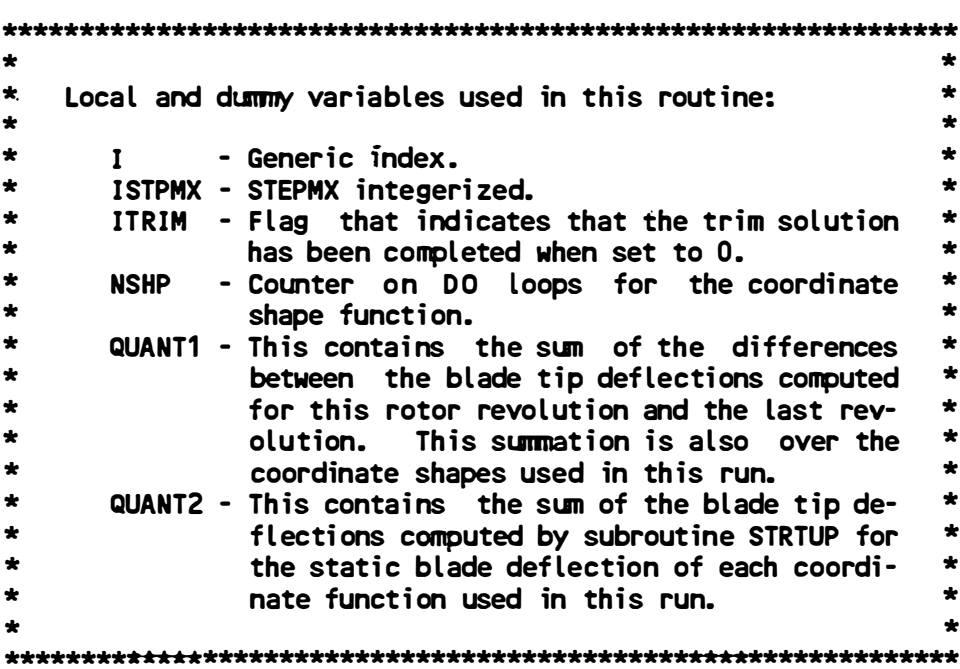

\begin{tabular}{ll} 
REAL & \multicolumn{1}{c}{ QUANT1 } \\
REAL & QUANT2 \\
INTEGER & I \\
INTEGER & ISTPMX \\
INTEGER & ITRIM \\
INTEGER & NSHP \\
INCLUDE & 'C:INCLUDEICONST2.INC' \\
INCLUDE & 'C:INCLUDE ILIMITE.INC' \\
INCLUDE & 'C:INCLUDEISARAYS.INC' \\
INCLUDE & 'C:INCLUDEISTART. INC' \\
INCLUDE & 'C:INCLUDEITURBN.INC'
\end{tabular}

Integerize the STEPMX interval.

ISTPMX $=$ RAD2DG*STEPMX +0.001

C Compute the sum of the tip deflections that were computed in C subroutine STRTUP.

QUANT2 $=0.0$

DO 100 NSHP $=1$, NSHAPS

100 QUANT2 = QUANT2 + SO(NSHP)

QUANT2 = ABS( QUANT2 )

C

Check each STEPMX station around the disk.

DO 210 I=ISTPMX, 360, ISTPMX

QUANT1 $=0.0$ 
C Compute the sum of the differences between tip deflections

C for this rotor revolution and the last.

DO 200 NSHP $=1$, NSHAPS

QUANT1 = QUANT1 + SNEW(NSHP, O,I) - SOLD(NSHP,I)

Compare the trim error fraction to the current deflection differences. If any station fails the test, return for another revolution.

IF( ABS( QUANT1 ).GT: ( 0.01*QUANT2*TRMERR ) ) RETURN

210 CONTINUE

C All stations satisfied the trim error criterion. The trim solution
C is now complete. Set the ITRIM flag to zero to signal it. Loads

C can now be computed and the yaw run started.

ITRIM $=0$

RETURN

END

FUNCTION TRPZOD ( LOWLIM , BLTIP , FOF , NPTS )

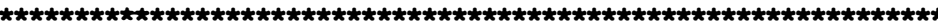

Function TRPZOD performs composite trapezoidal integration on a set of data points transmitted from the $*$ calling routine. For derivation of the formula and * limitations, see Carnahan, p. 78 (see full reference in * corments for function SIMPSN). For computational efficiency, the interval width is not used in the formulation until the end when it is multiplied by the sum.

This function is similar to function TRAP in Module 1 of the STRAP code.

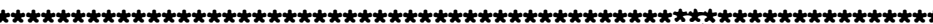

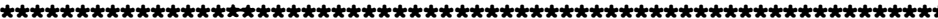

External references in this routine:

none

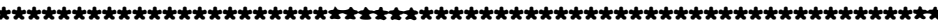

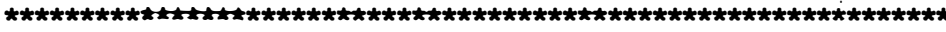

Named COMMON blocks used in this routine:

none

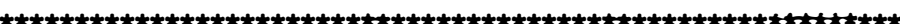

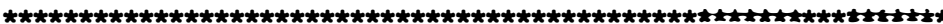

Local and dumy variables used in this routine:

BLTIP - Blade length. All integrations using this function are performed from the lower limit indexed by LOWLIM to the upper limit BLTIP.

FOF - Array of data points to be integrated. They are treated by this function as the value of a function evaluated at specific points.

H - Subinterval length given by $H=(B-A) / N$.

I LOWLIM - Index into the FOF array. blade position indexed by LOWLIM is given by BLTIP*(LOWLIM-1)/(NPTS-1).

NPTS - Number of points along the blade used to perform simpson's integration for calculat- 


$\begin{array}{ll}\text { REAL } & \text { BLTIP } \\ \text { REAL } & \text { FOF (201) } \\ \text { REAL } & \text { H } \\ \text { REAL } & \text { TRPZOD } \\ & \\ \text { INTEGER } & \text { I } \\ \text { INTEGER } & \text { LOWLIM } \\ \text { INTEGER } & \text { NPTS }\end{array}$

IF ( LOWLIM .EQ. NPTS ) THEN

TRPZOD $=0.0$

RETURN

END IF

C

$$
H=B L T I P /(N P T S-1)
$$

C

C

C

$$
\text { DO } 10 \quad I=\text { LOWLIM+1, NPTS }-1
$$

10 TRPZOD $=$ TRPZOD + FOF $(1)$

C 20 TRPZOD $=H * T R P Z O D$

RETURN

END

SUBROUTINE VREL ( INBORD，NPTS , BLDANG )

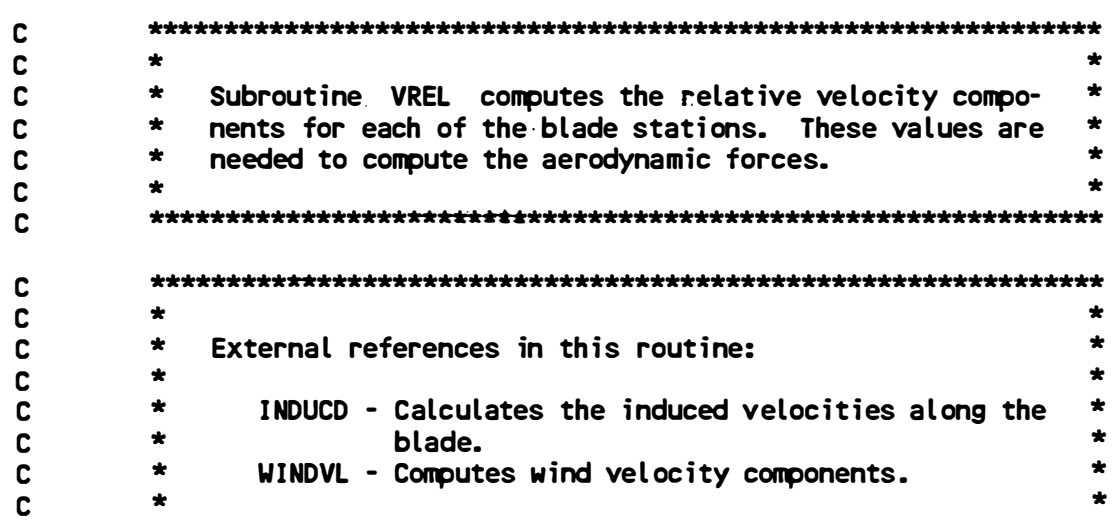




\begin{tabular}{|c|c|}
\hline $\begin{array}{l}\text { INTEGER } \\
\text { INTEGER } \\
\text { INTEGER }\end{array}$ & $\begin{array}{l}\text { I } \\
\text { INBORD } \\
\text { NPTS }\end{array}$ \\
\hline INCLUDE & 'C:INCLUDE \BLADE2. INC' \\
\hline INCLUDE & 'C:INCLUDE\CONST2.INC' \\
\hline INCLUDE & 'C:INCLUDE JDELTV.INC' \\
\hline INCLUDE & 'C:INCLUDE JFORMS.INC' \\
\hline INCLUDE & 'C:INCLUDEXPOSITN.INC' \\
\hline INCLUDE & 'C:INCLUDE \TURBN.INC' \\
\hline INCLUDE & 'C:INCLUDEIVINDUC. INC' \\
\hline INCLUDE & 'C:INCLUDEIVREL1. INC' \\
\hline INCLUDE & 'C:INCLUDE \WNDVEL. INC' \\
\hline
\end{tabular}

C Compute the wind velocity components and then the induced

C velocity components.

CALL WINDVL ( NPTS , BLDANG )

CALL INDUCD ( INBORD， NPTS , BLDANG )

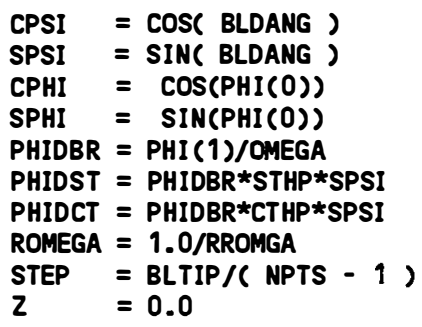

DO $100 \mathrm{I}=1$, NPTS

$$
R=\text { HUBRAD }+2
$$

C Clear the velocity components on the nonaerodynamic portion

IF ( I .LT. INBORD ) THEN

$$
\begin{aligned}
& \operatorname{VETA}(I)=0.0 \\
& \operatorname{VZETA}(I)=0.0
\end{aligned}
$$

ELSE

$$
X \quad=R / R R
$$

VETA(I) $=-X *$ CTHC

\&

$+V 1(1,1) *$ ROMEGA*STHP

$\operatorname{VZETA}(I)=-X * S T H C-\operatorname{VE}(1,1) *$ ROMEGA

\&

- V1 $(1,1) *$ ROMEGA*CTHP

\&

QUANT1 = VINDR(I ) + ROMEGA*( VWIND(I) - HUBVEL ) + ROMEGA*DELTVY(I) - DVIND(I)

QUANT2 = DELTVX(I)*ROMEGA*CPHI + SPHI*QUANT1

QUANT3 = PHIDBR*( ABAR + BETAO*X)

QUANT4 = -DELTVZ (I)*ROMEGA

\& $+\mathrm{CHI} * \mathrm{VINDR}(I) * C P H I$

$\operatorname{VETA}(I)=\operatorname{VETA}(I)-$ QUANT 1*STHC*CPHI 


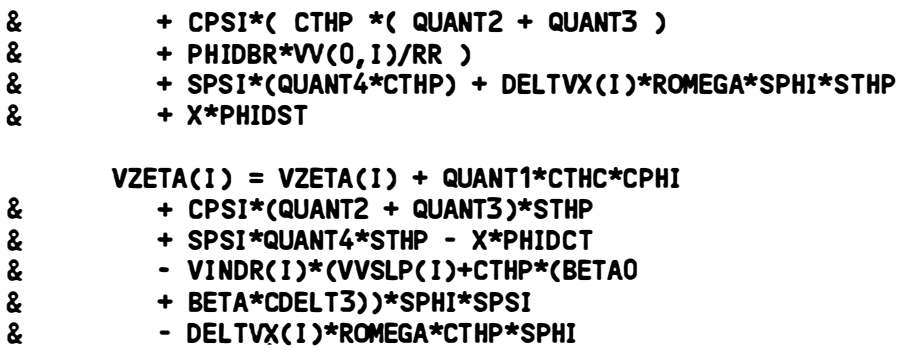

Convert the velocity components into dimensional form before returning to the calling routine. The VEta term is negated to account for the sign convention that positive velocities are from the leading edge to the trailing edge.

VETACI $)=-V E T A(I) * R R O M G A$

END IF

C Increment the location along the blade and make another pass.

$Z=Z+$ STEP

100 CONTINUE

-RETURN

END

SUBROUTINE WINDVL ( NPTS , BLDANG )

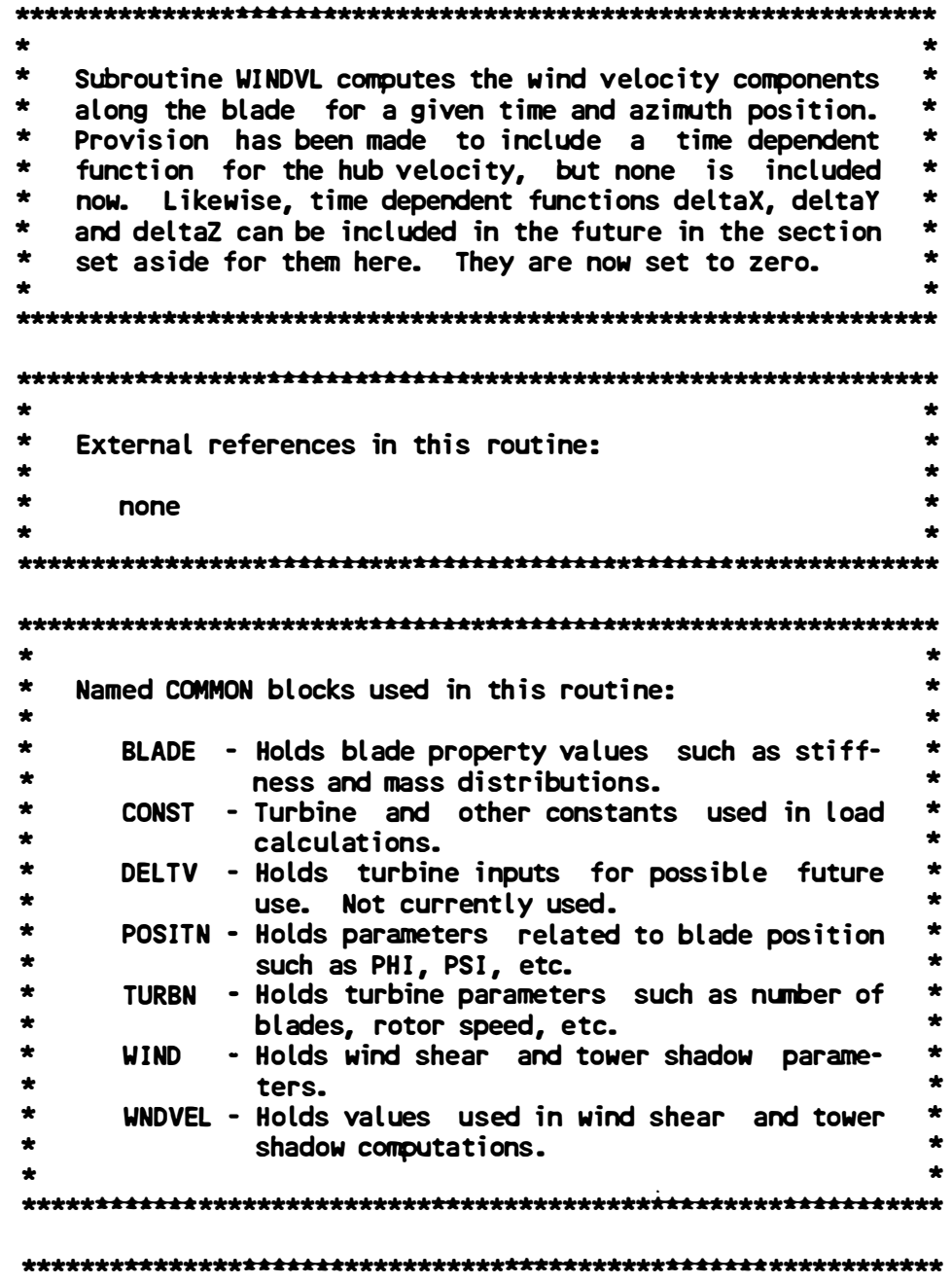




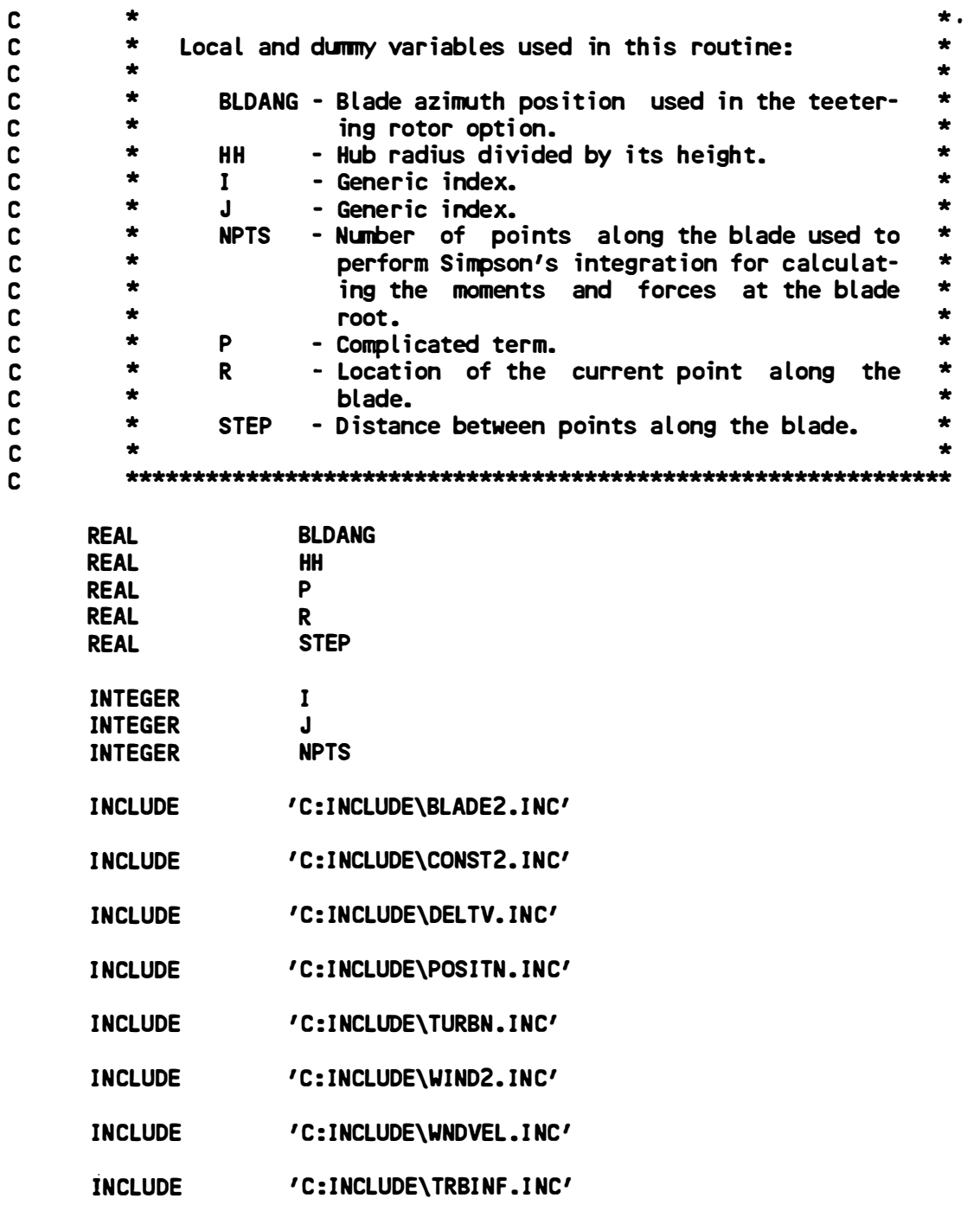

C Compute the wind velocity at the hub. A time dependent function C may be added in the future.

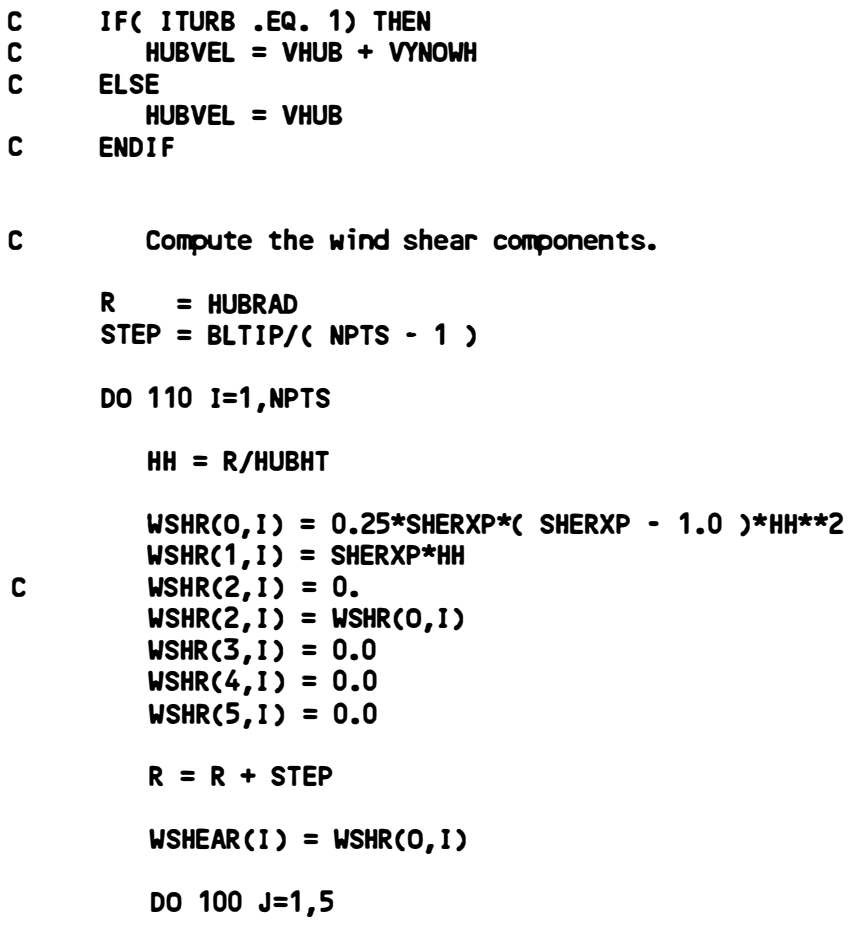


110 CONTINUE

C Compute tower shadow effects. When blade \#1 is close to 360

C degrees azimuth, the second blade must know that it is in the

C tower shadow.

IF ( ABS( BLDANG - PSISHD) .LE. PSIZER ) .THEN

P = KSHADW*PI/PSIZER

TSHADW $=$ TSUBO + TSUBP*COS( $P *($ BLDANG - PSISHD $))$

ELSE IF ( ABSC BLDANG - PSISHD - 2*PI ) .LE. PSIZER ) THEN

$P \quad=$ KSHADW*PI/PSIZER

TSHADW $=$ TSUBO + TSUBP*COS( $P *($ BLDANG - PSISHD $-2 * P I))$

ELSE

TSHADW $=0.0$

END IF

DO $200 I=1$,NPTS

$\operatorname{VWIND}(I)=$ HUBVEL *( $1.0+\operatorname{WSHEAR}(I)-$ TSHADW $)$

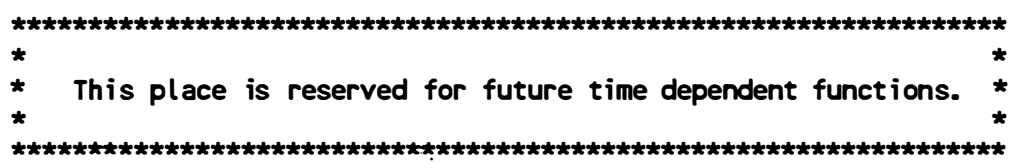

C Input the time dependent windspeed fluctuations if

C working on a turbulent analysis: ITURB .EQ. 1

$R=H U B R A D /(H U B R A D+B L T I P)$

STEP $=(1 .-R) /($ NPTS -1$)$

C Flag for deciding if working on a turbulence analysis.

IF( ITURB .EQ. 1 ) THEN

C Work on blade \#2 first.

IF( BLDANG .EQ. PSI(1)+PI) THEN

DO $500 I=1$, NPTS

C Linearly interpolate to get windspeed values at

C intermediate blade radial stations.

IF(R .LE. 0.5) THEN

DELTVY $(I)=(V Y N O W 2(1)-V Y N O W H) * 2 . * R+V Y N O W H$

ELSE

\&

$\operatorname{DELTVY}(1)=(V Y N O W 2(2)-V Y N O W 2(1)) * 2 . *(R-.5)$

$500 \quad$ R=R+STEP

500 CONTINUE

C Same procedure for blade \#1.

ELSEIF ( BLDANG .EQ. PSI(1)) THEN

DO $600 \mathrm{I}=1$,NPTS

IF(R . LE. 0.5) THEN

DELTVY (I) $=($ VYNOW1 (1) $-V Y N O W H) * 2 . * R+V Y N O W H$

ELSE

\&

$\operatorname{DELTVY}(1)=(\operatorname{VYNOW1}(2)-\operatorname{VYNOW1}(1)) * 2 . *(R-.5)$

+ VYNOW1(1) 


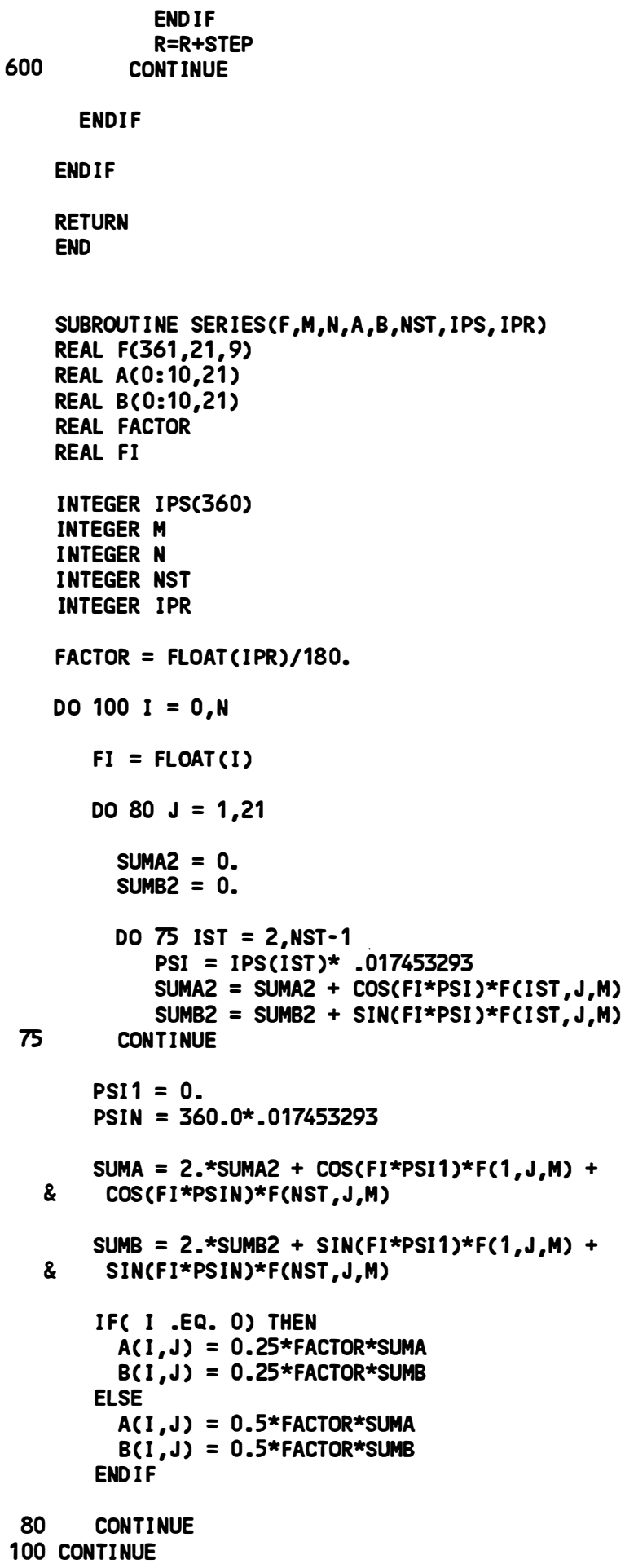


center of the hub.

Before this windspeed data is read in, the user is asked for the name of the file containing the turbulent windspeed data, is asked whether this data is in metric (meters/sec) or english units $(\mathrm{ft} / \mathrm{s})$ and is asked for the name of the file to contain the predicted turbulent loads data. For the structure of this file, see the block of comments near the WRITE statement toward the end of this subroutine.

BE SURE TO RUN THE TRIM SOLUTION CORRESPONDING WITH THIS TURBULENCE ANALYSIS WITH A VALUE OF SHERXP EQUAL TO ZERO IF THE VEERS GENERATED WINDSPEED DATA ALREADY HAS THE EFFECTS OF WINDSHEAR. IF YOU DON'T THEN THE PREDICTIONS WILL HAVE TO MUCH CYCLIC CONTENT, DUE TO TWO TIMES THE DESIRED WINDSHEAR VALUES.

REAL STEMP
REAL ANGLE
REAL AZIDAT
REAL AZIINC
REAL AZIOLD
REAL BLDANG
REAL DELVY1
REAL DELVY2
REAL HUBLOD
REAL PSIDIF
REAL RESLTS
REAL SHFLOD
REAL TEETER
REAL VY1
REAL VY2
REAL VYHUB
REAL VYSAV1
REAL VYSAV2 (
REAL VYSAVH (
INTEGER I
INTEGER J
INTEGER NEW
INTEGER NPTS
INTEGER NSHP
INTEGER OLD

CHARACTER *50 WNDFIL

CHARACTER $* 50$ LODFIL

CHARACTER * 2 ANS

$\begin{array}{ll}\text { INCLUDE } & \text { 'C:INCLUDEIBLADE2. INC' } \\ \text { INCLUDE } & \text { 'C:INCLUDEILIMITC.INC' } \\ \text { INCLUDE } & \text { 'C:INCLUDEIPOSITN.INC' } \\ \text { INCLUDE } & \text { 'C:INCLUDEISARAYS. INC' } \\ \text { INCLUDE } & \text { 'C:INCLUDEITURBN.INC' } \\ \text { INCLUDE } & \text { 'C:INCLUDEITRBINF.INC' } \\ \text { INCLUDE } & \text { 'C:INCLUDEICONST2.INC' } \\ \text { INCLUDE } & \text { 'C:INCLUDE IDELTV.INC' } \\ \text { INCLUDE } & \text { 'C:INCLUDEIWIND2.INC' } \\ \text { SAVE } & \text { NEW } \\ \text { SAVE } & \text { OLD } \\ \text { SAVE } & \text { STEMP } \\ & \\ \text { DATA } & \text { NEW / } 1 / \\ \text { DATA } & \text { OLD / } /\end{array}$

PRINT *, , ,

PRINT *', 'Turbulence Analysis Run Set-up'

PRINT *, ',

C Read in the name of the file containing the VEERS generated

C windspeed data.

PRINT *, 'Read in the name of the wind residual time series file' READ *, WNDFIL

PRINT *, WNDFIL

C See if the windspeed data is in metric or english units. 
PRINT *, 'Is the wind data in metric units (meters/sec)?'

READ *, ANS

PRINT *, ANS

PRINT *, '

C Give the name of the file you want to write rotor and blade

C teeter, loads, shaft-loads, etc. out to.

PRINT *, 'Read in the name of the file for loads output'

READ * LODFIL

PRINT *, LODFIL

OPEN (15, FILE = WNDFIL, STATUS = 'UNKNOWN')

OPEN (16, FILE = LODFIL, STATUS = 'UNKNOWN')

NSCN $=1$

AZIDAT $=0$.

AZIDAT is the azimuth angle read from data,

VYI( $\mathrm{J}$ ) is the windspeed data

for blade number 1, and VY2(J), is the windspeed data

for blade number 2, and VYHUB is the hubcenter windspeed.

Currently MSTAT is limited to 2 . We usually read in windspeed

data at the $50 \%$ and $100 \%$ rotor radius locations, for each

blade and then the hubcenter windspeed.

Set the shear exponent from the trim run to

zero, since the file generated from the

VEERS model already has the effects of windshear

in it. Failure to do so will result in too much

windshear. Other parameters such as tower shadow

inputs are not set to zero unless the user

reruns the trim solution with zero values

for TP, TO - the tower shadow parameters.

Initialize values for SHEREXP, PSI $(0)$,

AZIOLD, ERROR, DELPSI(1),

STEMP, etc.

SHERXP $=0$.

PSI $(0)=0$

AZIOLD $=-0.0001$

ERROR $=0$.

$\operatorname{DELPSI}(1)=$ STEPMN

AZIINC = TIMINC*OMEGA

set the initial blade deflection, velocity, and acceleration values to what was calculated in the trim solution at zero azimuth angle. This serves to start the solution process.

DO 260 NSHP $=1$, NSHAPS

DO $250 \mathrm{I}=0,2$

STEMP(NSHP,OLD,I) = SNEW(NSHP, I , 360) CONTINUE

Set the left-hand endpoint windspeed value needed in the linear interpolation process equal to VHUB since we have not read in a windspeed data line from the VEERS file yet. The right-hand endpoint value will be the new line of windspeed data read in.

These values are necessary in order to have endpoints for interpolation, since the blade azimuth in the numerical integration process will lie between azimuth values read from the data (AZIDAT). Setting this first lefthand point to VHUB starts the process. I=0 stands for the left-hand endpoint value, while $I=1$ is the right-hand endpoint. VYSAV1 is for blade 1, VYSAV2 is for blade 2 , and VYSAVH is the hub-center. $J$ is equal to 1 or 2 depending on whether it's the 50\% station or the blade tip. 
IF (( ANS .EQ. ' $Y$ ') .OR. ( ANS .EQ. ' $Y$ ')) THEN

DO $220 \mathrm{~J}=1$, MSTAT

VY1 (J) $=3.28 * V Y 1(J)$

$\operatorname{VY2}(J)=3.28 * V Y 2(J)$ CONTINUE

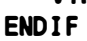

C

Convert AZIDAT to radians

AZIDAT = AZIDAT/RADZDG

IF ( AZIDAT .EQ. O.) THEN ENDIF

PSI $(0)=6.283185-A Z I$ INC

Be sure that the windspeed data produced by the VEERS model contains the hubheight or mean windspeed. If it doesn't then the next 4 lines of code should be commented out.

The purpose of the next 4 lines of code is

C to subtract out the mean windspeed from the data input.

DO $230 \mathrm{~J}=1$, MSTAT

VY1 (J) $=$ VY1(J)-VHUB

230 CONTINUE

$\operatorname{VY2}(J)=\operatorname{VY2}(J)-V H U B$

VYHUB = VYHUB-VHUB

C We read in one line of windspeed data at a time.

We interpolate the windspeed data to get turbulent

windspeed fluctuations when the blade azimuth angle

lies between successive values in the input file.

350

Set the, right-hand endpoint windspeed in the interpolation process equal to the windspeeds just read in.

DO $350 \mathrm{~J}=1$, MSTAT

$\operatorname{VYSAV1}(1, J)=\operatorname{VY} 1(\mathrm{~J})$

$\operatorname{VYSAV} 2(1, J)=\operatorname{VY2}(\mathrm{J})$

CONTINUE

$\operatorname{VYSAVH}(1)=$ VYHUB

If we have passed through 360 degrees

then subtract out 360 degrees from

PSI. We are not able to deal with

azimuth angles greater than 360 deg.

Be sure to convert to radians.

IF ( (AZIDAT - AZIOLD) .LT. O.) THEN

PSI $(0)=$ PSI $(0)-360 . / R A D 2 D G$

$A Z I O L D=A Z I O L D-360 . / R A D 2 D G$

ENDIF

C Find the next azimuth angle in the

C code numerical solution process ( not

C the azimuth angle of the next line

C of wind data. PSI (1) represents

C this new azimuth angle (radians).

C PSIDIF represents the difference between

$C$ this new azimuth location and the last

C azimuth angle read in from the data.

CALL NXTAZI ( AZIDAT)

PSIDIF $=$ PSI (1) $-A Z I O L D$ 


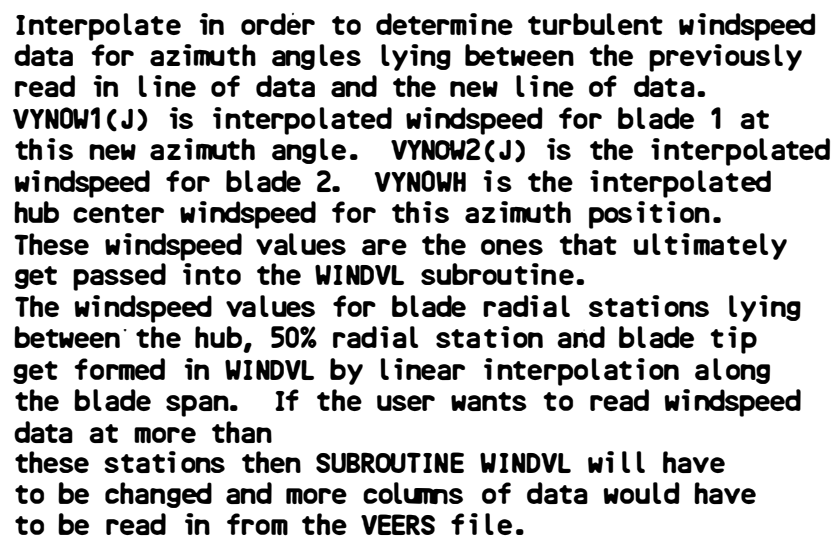

CALL EULER ( STEMP, NSHAPS, NPTS)

IF (( ERROR .GT. EUERR) .AND. (DELPSI(1) .GT. STEPMN)) GO TO 400

C Set the blade angle BLDANG equal to the C azimuth angle of blade \#2.

$$
\text { BLDANG }=\text { PSI }(1)+P I
$$

C First calculate blade loads for blade \#2.

C The response and load results for blade

C \#2 will be stored in the variable

C RESULTS(2,I,J)

\section{CALL LODCLC(BLDANG, STEMP, RESLTS)}

C Set the blade angle BLDANG equal to the

C azimuth angle for the first blade.

$$
\text { BLDANG }=\text { PSI (1) }
$$

C Calculate blade loads for blade \#1.

C The response and load results for blade

C \#1 will be stored in the variable

C results $(1, I, J)$. 
CALL LODCLC(BLDANG, STEMP, RESLTS)

'Calculate the shaft loads once the

C loads for blades \#1 and \#2 are known.

CALL SHAFT2(RESLTS, HUBLOD, SHFLOD, PSI )

This is the portion of the subroutine that writes out results to the user designated loads output file. ANGLE is the azimuth angle, TEETER is the rotor teeter angle (deg.), RESLTS $(1, I, J)$ is the results matrix for blade \#1 at the I'th station. $J$ corresponds to the results item to printed out: $J=1$ to 7 .

$\operatorname{RESLTS}(2, I, J)$ Is the results matrix for blade \#2. I corresponds to the number of the blade radial station, from 1 to 21 , with 1 at the root and 21 at the blade tip.

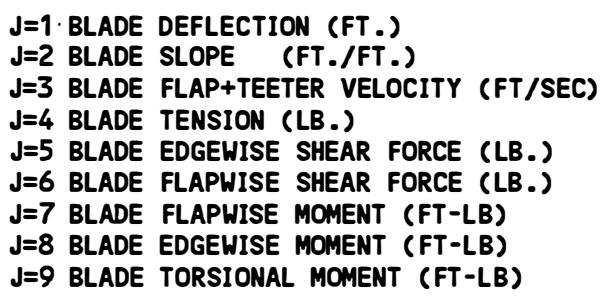

SHFLOD(1) IS THE SHAFT FORCE IN THE Xr DIRECTION. SHFLOD(2) IS THE SHAFT FORCE IN THE Yr DIRECTION. SHFLOD(3) IS THE SHAFT FORCE IN THE Zr DIRECTION. SHFLOD(4) IS THE SHAFT MOMENT ABOUT THE Xr AXIS. SHFLOD(5) IS THE SHAFT MOMENT ABOUT THE Yr AXIS. SHFLOD(6) IS THE SHAFT MOMENT ABOUT THE $\mathrm{Zr}$ AXIS.

Here we are printing out azimuth, teeter root $\mathrm{flap}$-bending for blades 1 and 2 , $65 \%$ span flap-bending for blade 1 , and shaft moments about the $X r, Y r$, and $\mathrm{Zr}$ axes. This statement will need to be changed by the user to write out items needed.
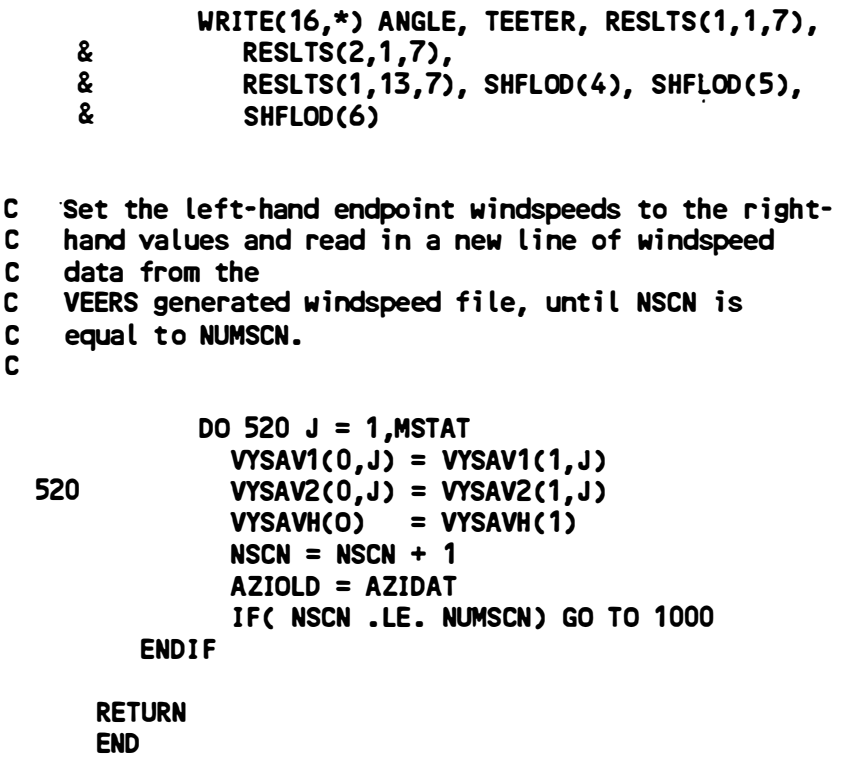

SUBROUTINE NXTAZI ( AZI ) 


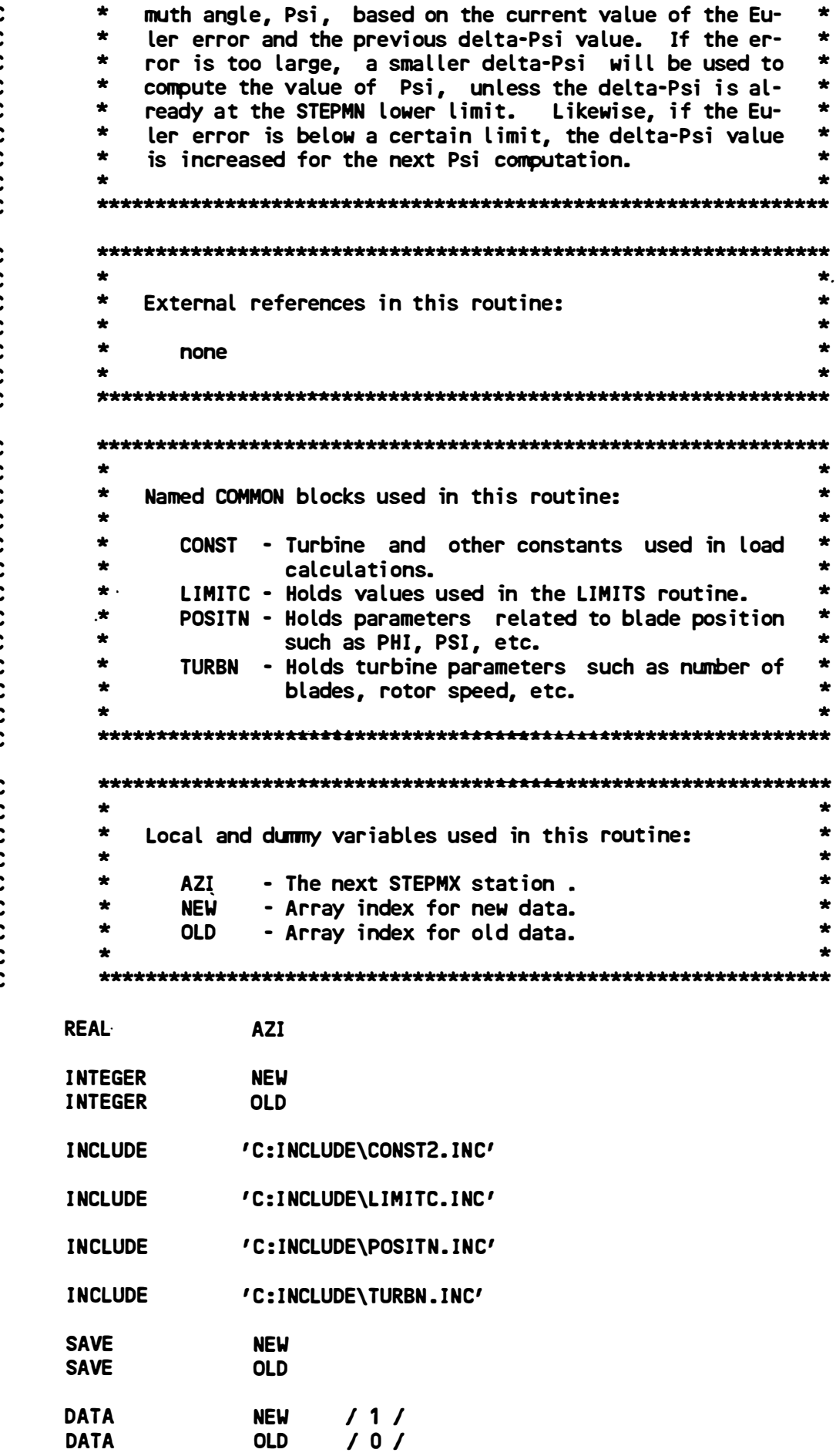

DATA OLD / $/$

Check the error. If it is too large, decrease delta-Psi by

C $50 \%$. If it is very small, increase delta-Psi by $50 \%$. The value of delta-Psi must always be greater than STEPMN.

IF ( ERROR . GT. EUERR ) THEN

DELPSI (NEW) $=\operatorname{AMAXI(0.5*DELPSI}(N E W)$, STEPMN )

ELSE IF ( ERROR - LT. 0.1*EUERR ) THEN

DELPSI $(N E W)=1.5 *$ DELPSI (NEW)

END IF 
This section of code checks the position of the new Psi against the next STEPMX station. If the next Psi will go past the STEPMX station, the delta-Psi is adjusted to bring the next Psi directly onto the STEPMX point. If the next Psi will fall just short of a STEPMX station, the delta-Psi is adjusted to assure that it will not be necessary to use a delta-Psi smaller than STEPMN to reach the next STEPMX station on the next iteration.

Programer note:

The following logic can produce a delta-Psi that is less than STEPMN for two steps. It would then move back within tolerances. This case comes up when we are within slightly less than two STEPMNs of the next STEPMX station. This algorithm cannot produce a delta-Psi that is less than STEPMN/2.

MLB

IF ( PSI (OLD)+DELPSI (NEW) .GT. AZI ) THEN

DELPSI (NEW) $=$ AZI - PSI (OLD)

ELSE IF ( PSI(OLD)+DELPSI(NEW)+STEPMN .GT. AZI ) THEN

IF ( (DELPSI (NEW) .NE. STEPMN) .OR. (ERROR .LE. EUERR) ) THEN

DELPSI(NEW) $=0.5 *(A Z I-P S I(O L D))$

END IF

END IF

Set new Psi and delta-Time.

PSI (NEW) = PSI (OLD) + DELPSI (NEW)

$\operatorname{DELTAT}($ NEW) $=$ DELPSI (NEW)/OMEGA

Get time values if we're working on a yawing solution.

IF ( ITRIM .EQ. 0 ) $\operatorname{TIME(NEW)~=~TIME(OLD)~+~DELTAT(NEW)~}$

RETURN

END

SUBROUTINE LODCLC( BLDANG, STEMP, RESLTS)

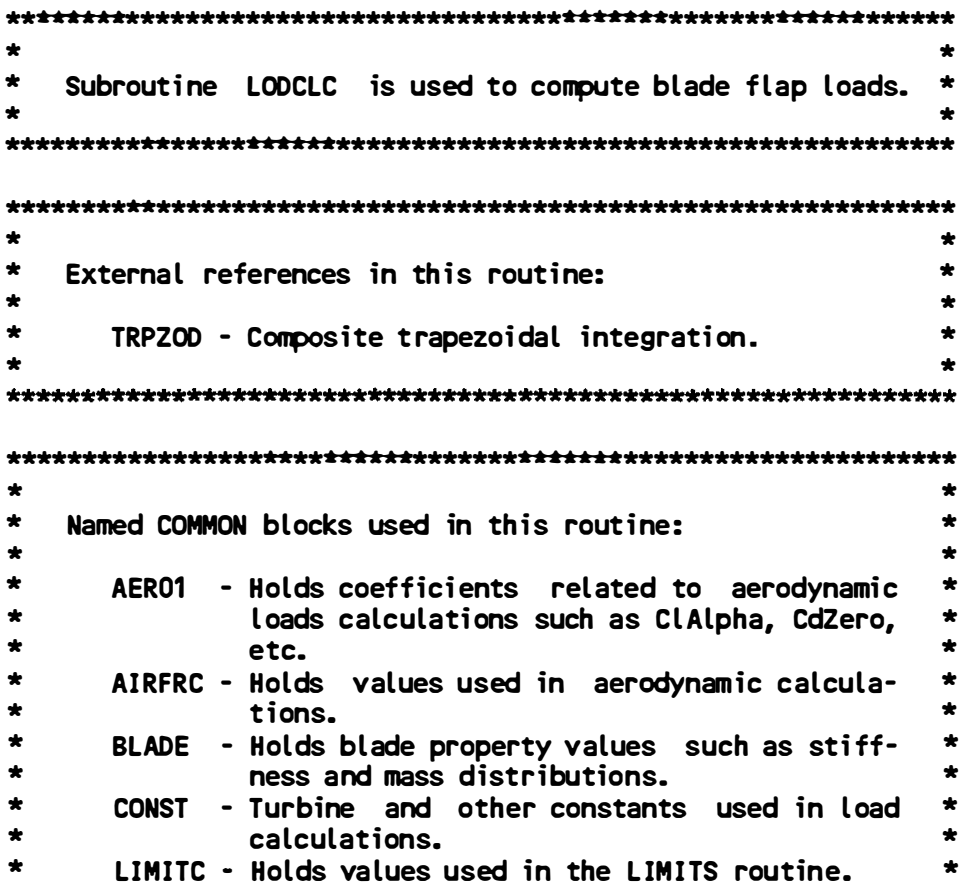




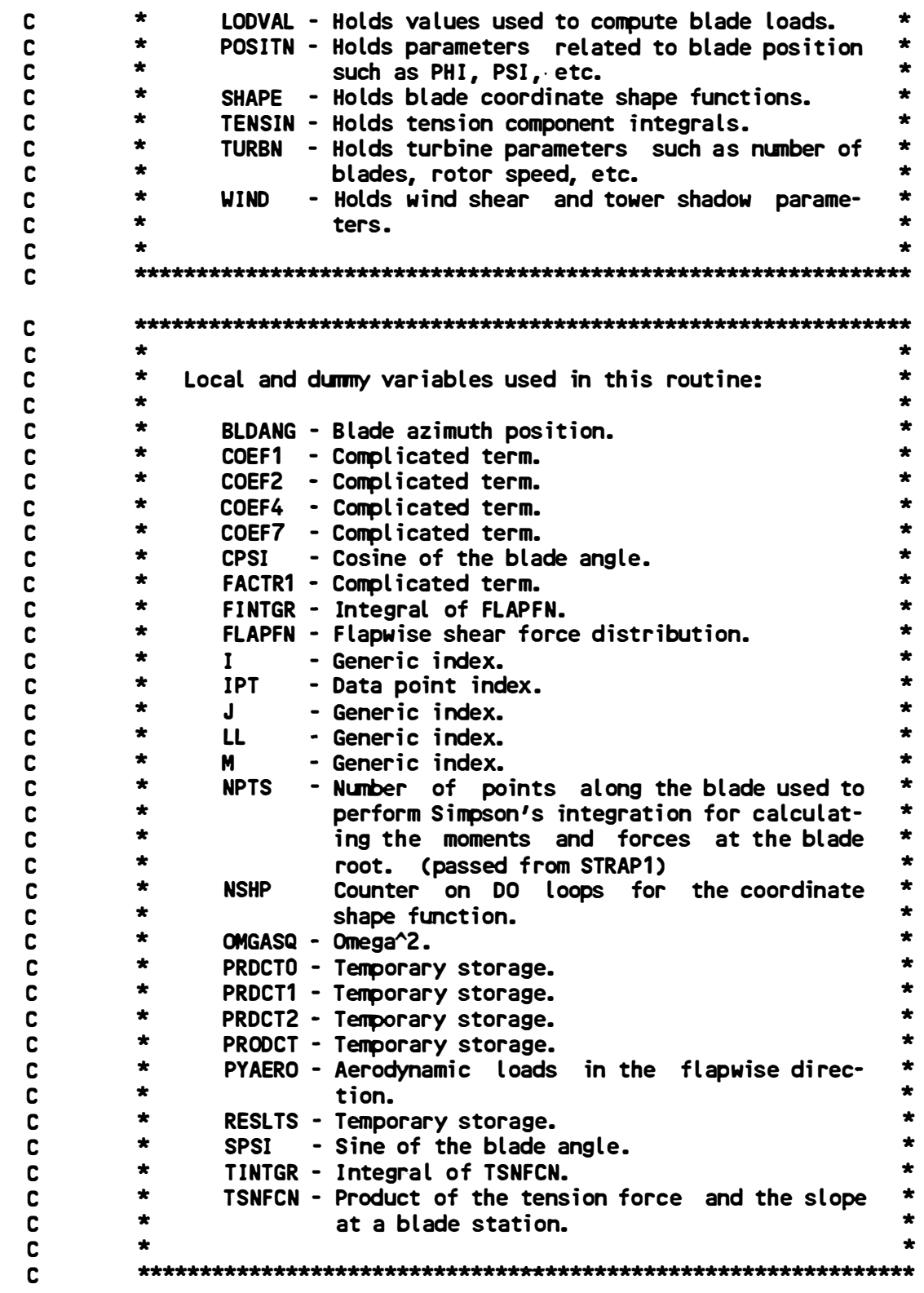

$\begin{array}{ll}\text { REAL } & \text { BLDANG } \\ \text { REAL } & \text { COEF1 } \\ \text { REAL } & \text { COEF2 } \\ \text { REAL } & \text { COEF4 } \\ \text { REAL } & \text { COEF7 } \\ \text { REAL } & \text { CPSI } \\ \text { REAL } & \text { FACTR1 } \\ \text { REAL } & \text { FINTGR } \\ \text { REAL } & \text { FLAPFN (21) } \\ \text { REAL } & \text { EDGEFN (21) } \\ \text { REAL } & \text { TSNFCN (21) } \\ \text { REAL } & \text { VXFCN (21) } \\ \text { REAL } & \text { OMGASQ } \\ \text { REAL } & \text { PRODD } \\ \text { REAL } & \text { PRDCTO } \\ \text { REAL } & \text { PRDCT1 } \\ \text { REAL } & \text { PRDCT2 } \\ \text { REAL } & \text { PRODCT (21) } \\ \text { REAL } & \text { PYAERO (21) } \\ \text { REAL } & \text { PXAERO (21) } \\ \text { REAL } & \text { QZFCN (21) } \\ \text { REAL } & \text { RESLTS (2,21,9) } \\ \text { REAL } & \text { SPSI } \\ \text { REAL } & \text { STEMP (4, 0:1, 0:2) } \\ \text { REAL } & \text { TINTGR } \\ \text { REAL } & \text { TRPZOD } \\ & \end{array}$




\begin{tabular}{|c|c|}
\hline $\begin{array}{l}\text { INTEGER } \\
\text { INTEGER } \\
\text { INTEGER } \\
\text { INTEGER }\end{array}$ & $\begin{array}{l}\text { IDBLD } \\
\text { IPT } \\
\text { NPTS } \\
\text { NSHP }\end{array}$ \\
\hline INCLUDE & 'C:INCLUDE\AER01.INC' \\
\hline INCLUDE & 'C:INCLUDEXAIRFRC. INC' \\
\hline INCLUDE & 'C:INCLUDE\BLADE2.INC' \\
\hline INCLUDE & 'C:INCLUDE ICONST2.INC' \\
\hline INCLUDE & 'C:INCLUDEILIMITC.INC' \\
\hline INCLUDE & 'C:INCLUDEYLODVAL. INC' \\
\hline INCLUDE & 'C:INCLUDEIPOSITN.INC' \\
\hline INCLUDE & 'C:INCLUDE\SHAPE.INC' \\
\hline INCLUDE & 'C:INCLUDEITENSIN2.INC' \\
\hline INCLUDE & 'C:INCLUDE \TURBN. INC' \\
\hline INCLUDE & 'C:INCLUDEXWIND2.INC' \\
\hline $\begin{array}{l}\text { SAVE } \\
\text { SAVE } \\
\text { SAVE }\end{array}$ & $\begin{array}{l}\text { NPTS } \\
\text { NEW } \\
\text { OLD }\end{array}$ \\
\hline $\begin{array}{l}\text { DATA } \\
\text { DATA }\end{array}$ & $\begin{array}{ll}\text { NPTS } & / 21 / \\
\text { NEW } & / 1 /\end{array}$ \\
\hline
\end{tabular}

C Initialize some constants

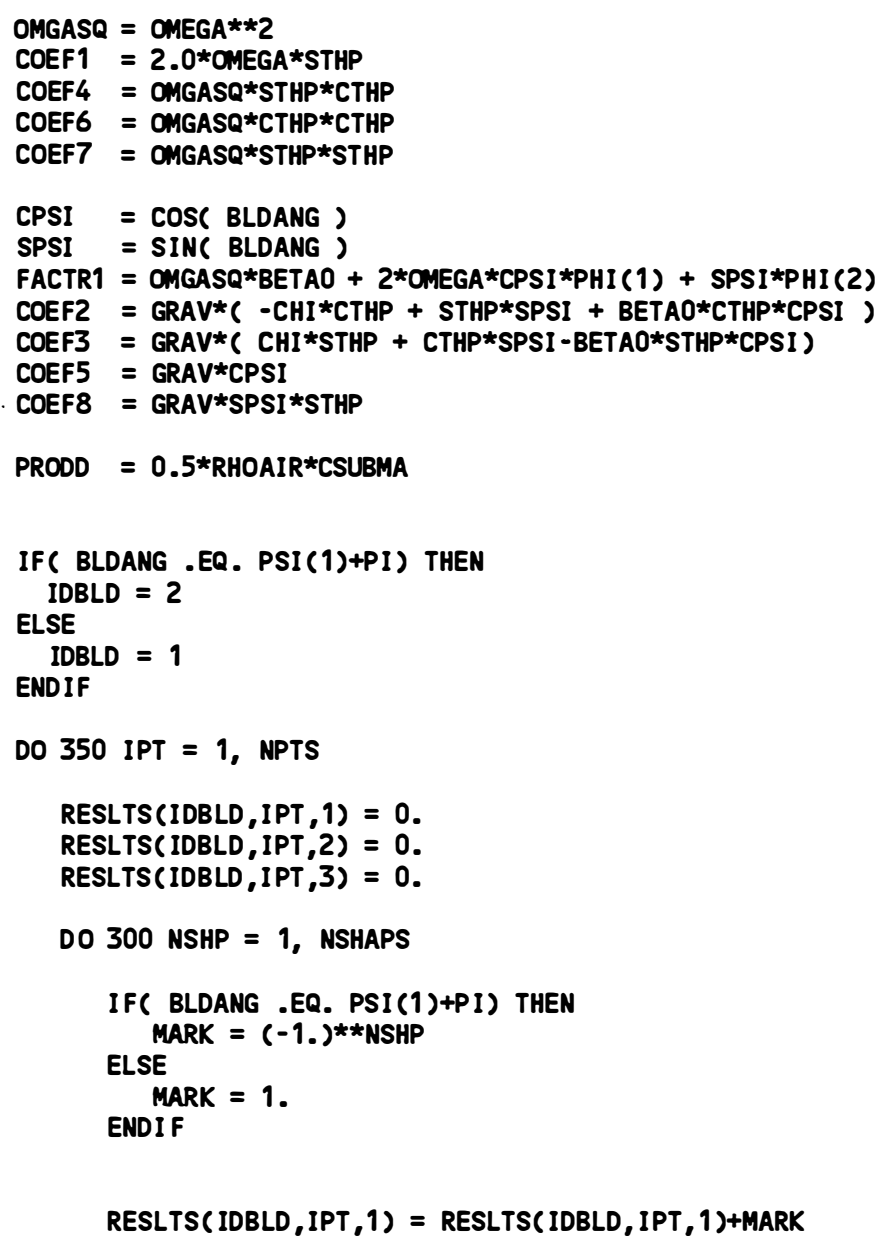




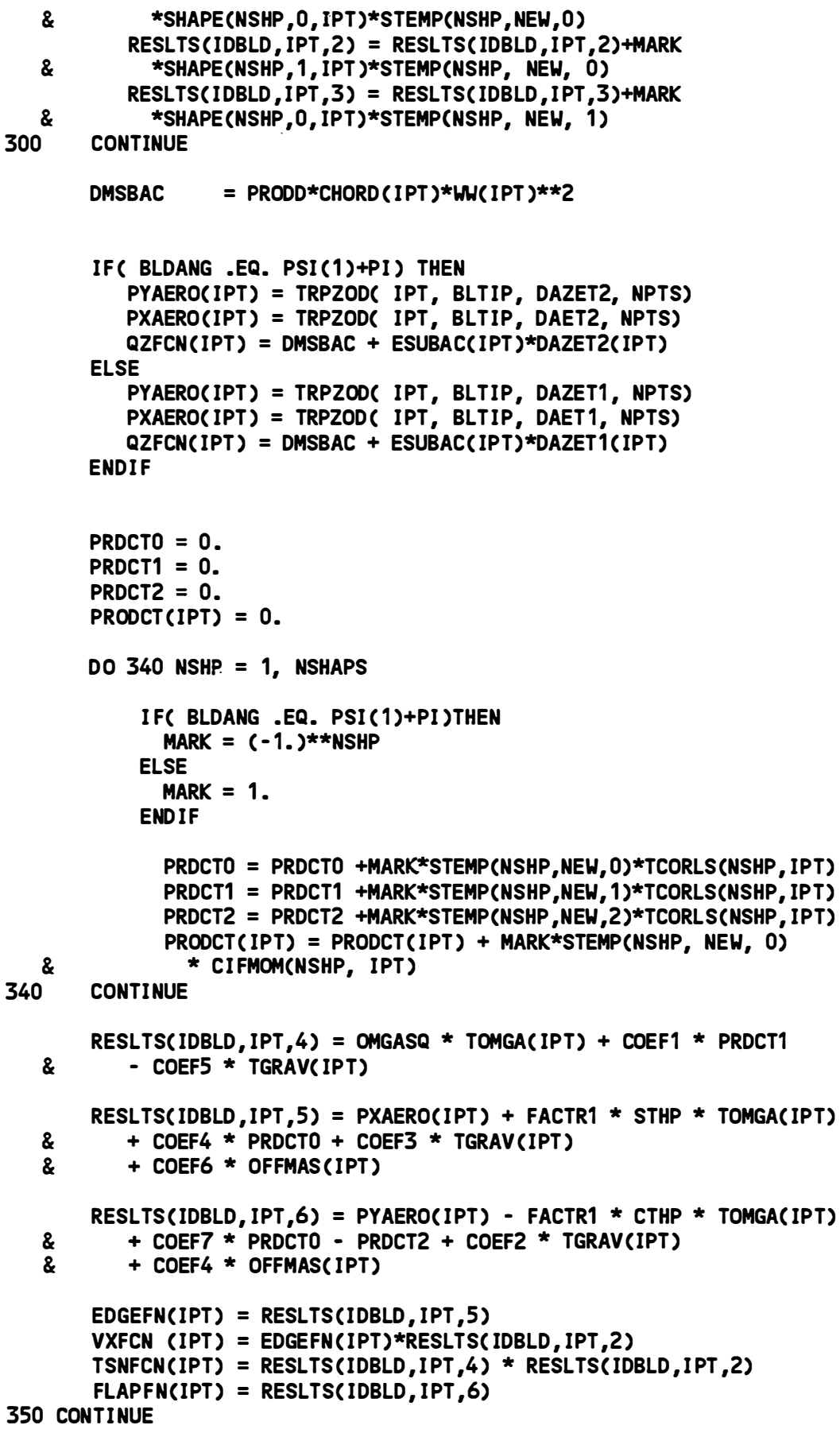

360 CONTINUE 
SUBROUTINE SHAFT(IPS, IPR,NSTN)

$\begin{array}{ll}\text { INCLUDE } & \text { 'C:INCLUDE ICONST2.INC' } \\ \text { INCLUDE } & \text { 'C:INCLUDE TTURBN.INC' } \\ \text { INCLUDE } & \text { 'C:INCLUDE RESLTS.INC' } \\ \text { INCLUDE } & \text { 'C:INCLUDE SSHFLOD.INC' } \\ \text { INCLUDE } & \text { 'C:INCLUDE BBLADE2.INC' } \\ \text { INCLUDE } & \text { 'C:INCLUDE IHUBPRP2.INC' }\end{array}$

CHARACTER * 50 SHFOUT

CHARACTER * 15 LABEL 1

CHARACTER * 10 LABEL2(6)

CHARACTER * 12 LABEL3(0:5)

CHARACTER * 12 LABEL4(0:5)

CHARACTER * 10 LABEL5(6)

INTEGER IPS(360)

INTEGER IPR

INTEGER NSTN

INTEGER NSTN1

INTEGER NST 1

INTEGER IST

INTEGER IST2

REAL A $(0: 10,6)$

REAL $B(0: 10,6)$

REAL $C(0: 10,6)$

REAL D $(0: 10,6)$

REAL SBETAO

REAL CBETAO

REAL FACTOR

REAL FI

REAL PSI 1

REAL PSIN

SAVE LABEL1

SAVE LABEL?

SAVE LABEL3

SAVE LABEL4

SAVE LABEL5

DATA LABEL1

\& / 'Azimuth Angle'/

DATA LABEL2

\& I 'FXr', 'Fyr',

\& 'Fzr', 'Mxí,',Myr', 'Mzr's

DATA LABEL3

\& / ' $\cos (0 *$ PSI )', ' $\cos (P S I) '$ '

$\&$ 'cos(2.*PSI)', $\cos (3 . \star P S I) '$ ',

\& $' \cos (4 . \star P S I) ', ' \cos (5 . \star P S I) ' \prime$

DATA LABEL4

\& $/$ 'sin( $\left.0 .{ }^{*} P S I\right) ', ' \sin (P S I) ', ' \sin \left(2 .{ }^{*} P S I\right) '$,

\& 'sin(3.*PSI)','sin(4.*PSI)','sin(5.*PSI)'/

DATA LABEL5

\& / 'Fxh', 'Fyh','Fzh',

\& 'Mxh', 'Myh','Mzh''

4000 FORMAT $(/, 1 \mathrm{X}, \mathrm{A}, 6(1 \mathrm{X}, \mathrm{A}))$

4200 FORMAT ( $/, 2 X, F 10.3,6(1 X, F 10.3))$

4300 FORMAT (//)

4400 FORMAT $(/, 17 X, 6(1 X, A))$

4500 FORMAT (/, 1X, A, 6(1X, F10.3))

SBETAO $=$ SIN(BETAO)

CBETAO $=\operatorname{COS}(B E T A O)$

PSI $1=0$.

PSIN $=360 . * 017453293$

FACTOR $=$ FLOAT $(I P R) / 180$. 


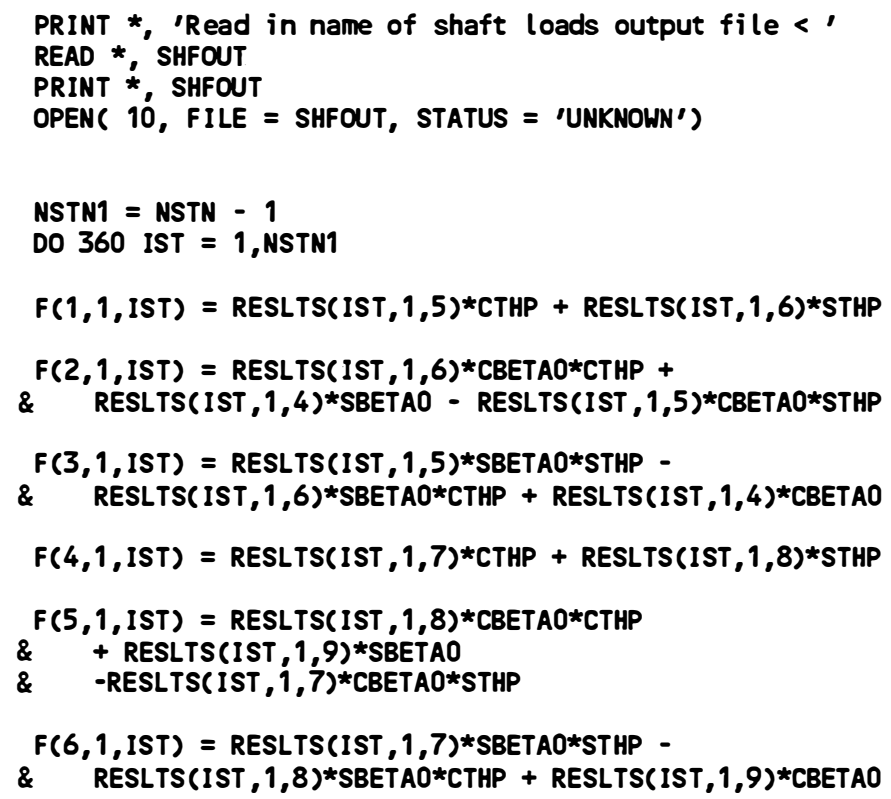

390 CONTINUE

DO $400 I=1,6$

$F(1,2$, NSTN $)=F(1,2,1)$

400 CONTINUE

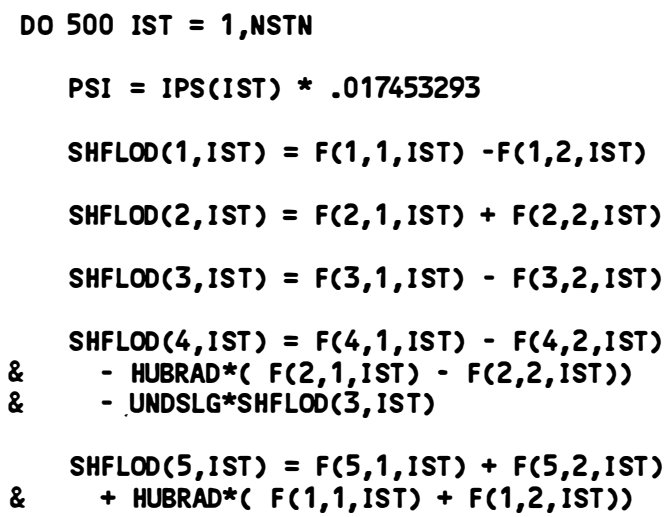


$\operatorname{SHFLOD}(6,1 S T)=F(6,1,1 S T)-F(6,2$, IST $)$

+ UNDSLG*SHFLOD $(1$, IST)

$\operatorname{HUBLOD}(1,1 S T)=\operatorname{SHFLOD}(1,1 S T) * \operatorname{COS}(P S I)+\operatorname{SHFLOO}(3,1 S T)$

$\star \operatorname{SIN}(P S I)$

$\operatorname{HUBLOO}(2$, IST $)=\operatorname{SHFLOO}(2$, IST $)$

HUBLOD( $3,1 S T)=\operatorname{SHFLOD}(3,1 S T) * \operatorname{COS}(P S I)-\operatorname{SHFLOD}(1,1 S T)$

$\star \operatorname{SIN}(P S I)$

$\operatorname{HUBLOD}(4,1 S T)=\operatorname{SHFLOD}(4,1 S T) * \operatorname{COS}(P S I)+\operatorname{SHFLOD}(6,1 S T)$

\&

* SIN(PSI)

$\operatorname{HUBLOO}(5,1 S T)=\operatorname{SHFLOO}(5,1 S T)$

$\operatorname{HUBLOO}(6,1 S T)=\operatorname{SHFLOD}(6,1 S T) * \operatorname{COS}(P S I)-$

\& $\operatorname{SHFLOD}(4,1 S T) * \operatorname{SIN}(P S I)$

500 CONTINUE

WRITE $(10, *)$ 'Low-Speed Shaft Loads in Rotor coordinates'

$\operatorname{WRITE}(10,4000)$ LABEL1, ( $\operatorname{LABEL2}(\mathrm{J}), \mathrm{J}=1,6)$

DO 420 IST $=1$, NSTN

WRITE $(10,4200)^{\prime}$ IPS(IST), (SHFLOO(J,IST), J=1,6)

420 CONTINUE

$\operatorname{WRITE}(10,4300)$

WRITE $(10, *)$ 'Hub loads in fixed frame hub coordinates'

WRITE(10,4000) LABEL1, ( LABEL5(J), J=1,6)

DO 430 IST $=1$, NSTN

$\operatorname{WRITE}(10,4200)$ IPS(IST), (HUBLOD(J,IST), J=1,6)

430 CONTINUE

C Compute harmonics of rotor shaft loads: Fourier Series expansion.

C Also compute harmonics of fixed frame hub loads, in hub coordinates.

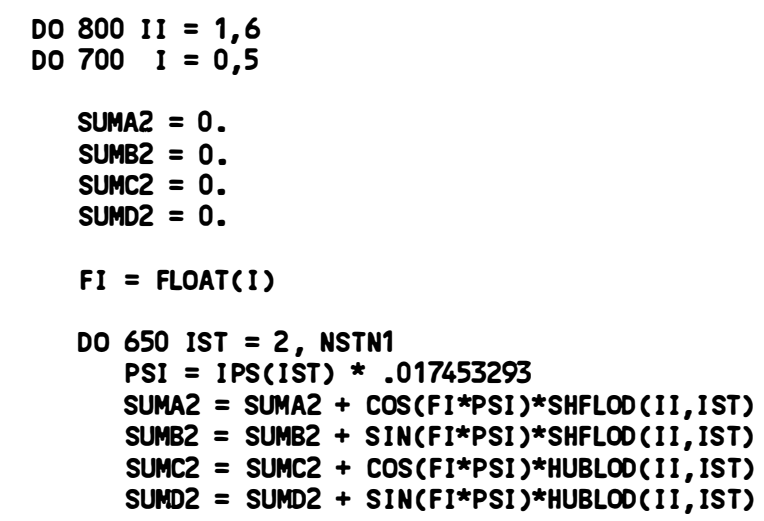

CONTINUE

\&

SUMA $=2 . *$ SUMA2 $+\operatorname{COS}(F I * P S I 1) * S H F L O D(I I, 1)+$ COS (FI*PSIN)*SHFLOD (II, NSTN)

SUMB $=2 . *$ SUMB2 + SIN(FI*PSI 1)*SHFLOD $(I I, 1)+$ SIN(FI*PSIN)*SHFLOD(II,NSTN)

SUMC $=2 . *$ SUMC2 $+\operatorname{COS}(F I * P S I 1) * H U B L O D(11,1)+$ COS(FI*PSIN)*HUBLOD (II,NSTN)

SUMD $=2 . *$ SUMD2 + SIN(FI*PSI 1)*HUBLOD $(I I, 1)+$

\& $\operatorname{SIN}(F I * P S I N) * H U B L O O(I I, N S T N)$

IF( I .EQ. 0) THEN

$A(I, I I)=0.25 *$ FACTOR*SUMA

$B(I, I I)=0.25 *$ FACTOR*SUMB

$C(I, I I)=0.25 *$ FACTOR*SUMC

$D(I, I I)=0.25 *$ FACTOR*SUMD

ELSE

D-105 
Appendix E

Modules 1 and 2 Flowcharts

E-1 


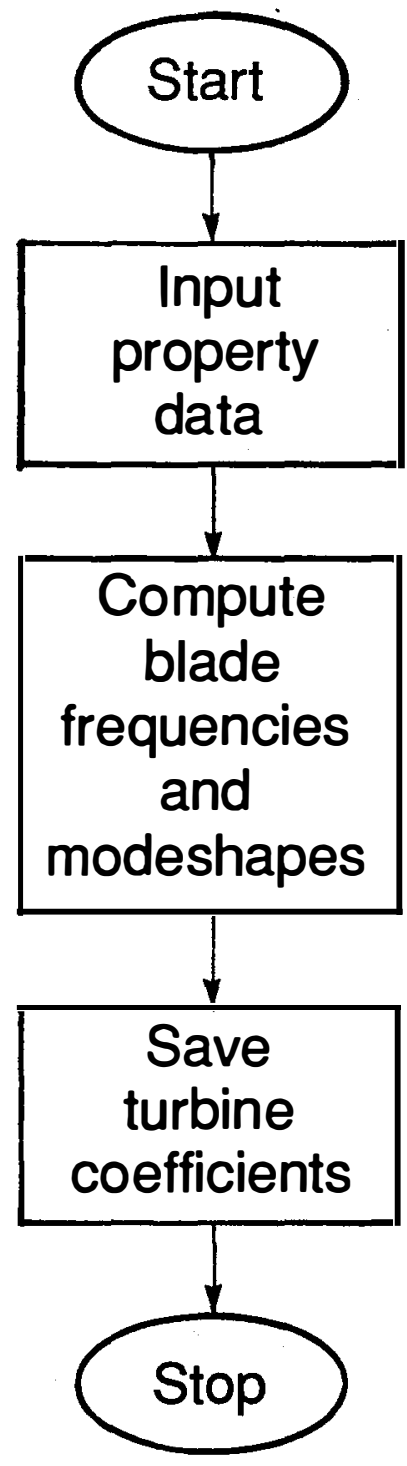

Figure E-1. Module 1 Flowchart 


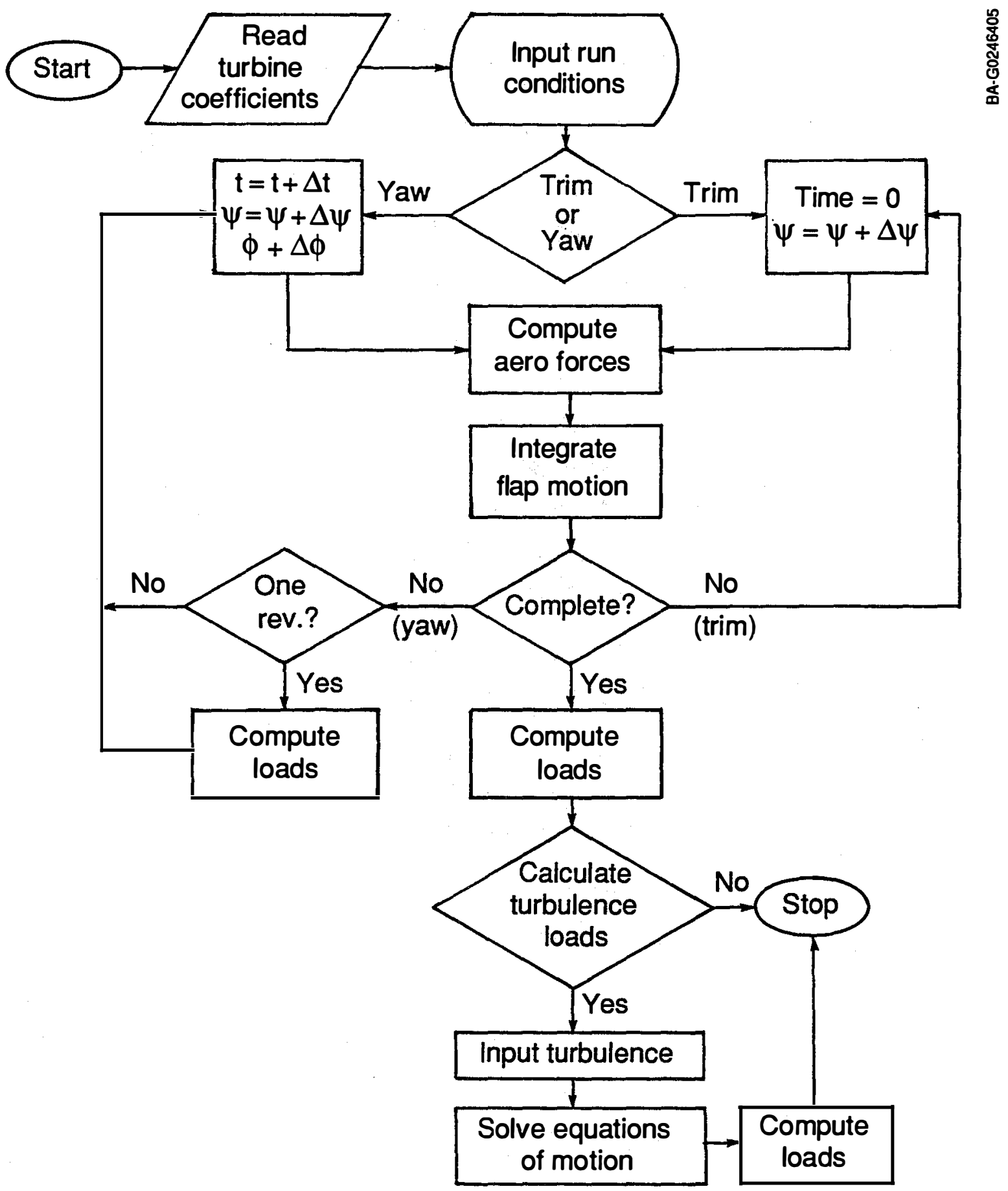

Figure E-2. Module 2 Flowchart 


\begin{tabular}{|c|c|c|c|}
\hline Document Control Page & $\begin{array}{l}\text { 1. NREL Report No. } \\
\text { NREL/TP-257-4671 }\end{array}$ & $\begin{array}{l}\text { 2. NTIS Accession No. } \\
\text { DE92010580 }\end{array}$ & 3. Recipient's Accession No. \\
\hline \multirow{2}{*}{\multicolumn{3}{|c|}{$\begin{array}{l}\text { 4. Title and Subtitle } \\
\text { User's Guide for the NREL Teetering Rotor Analysis Program (STRAP) }\end{array}$}} & $\begin{array}{l}\text { 5. Publication Date } \\
\text { August } 1992\end{array}$ \\
\hline & & & 6. \\
\hline \multicolumn{3}{|l|}{$\begin{array}{l}\text { 7. Author(s) } \\
\text { Alan D. Wright }\end{array}$} & $\begin{array}{l}\text { 8. Performing Organization Rept. No. } \\
\text { NREL/TP-257_4671 }\end{array}$ \\
\hline \multirow{2}{*}{\multicolumn{3}{|c|}{$\begin{array}{l}\text { 9. Performing Organization Name and Address } \\
\text { National Renewable Energy Laboratory } \\
1617 \text { Cole Blvd. } \\
\text { Golden, CO } 80401\end{array}$}} & $\begin{array}{l}\text { 10. Project/TaskWork Unit No. } \\
\text { WE21.8202 }\end{array}$ \\
\hline & & & $\begin{array}{l}\text { 11. Contract (C) or Grant (G) No. } \\
\text { (C) } \\
\text { (G) }\end{array}$ \\
\hline \multirow{2}{*}{\multicolumn{3}{|c|}{$\begin{array}{l}\text { 12. Sponsoring Organization Name and Address } \\
\text { National Renewable Energy Laboratory } \\
1617 \text { Cole Blvd. } \\
\text { Golden, CO } 80401\end{array}$}} & $\begin{array}{l}\text { 13. Type of Report \& Period Covered } \\
\text { Technical Report }\end{array}$ \\
\hline & & & 14. \\
\hline \multicolumn{4}{|l|}{ 15. Supplementary Notes } \\
\hline \multicolumn{4}{|c|}{$\begin{array}{l}\text { This Report gives the reader an overview of and instructions on the proper use of the National Renewable Energy Laboratory } \\
\text { (formerly the Solar Energy Research Institute, or SERI) Teetering Rotor Analysis Program (STRAP version 2.2). STRAP is a } \\
\text { derivative of the Force and Loads Analysis Program (FLAP). It is intended as a tool for prediction of rotor and blade loads and } \\
\text { response for only two-bladed teetering hub wind turbines. The effects of delta-3, undersling, hub mass, and wind turbulence are } \\
\text { accounted for. The objectives of this report are to give an overview of the code and also show the methods of data input and } \\
\text { correct code execution steps in order to model an example two-bladed teetering hub turbine. A large portion of the discussion } \\
\text { (Sections } 6.0,7.0 \text {, and } 8.0 \text { ) is devoted to the subject of inputing and running the code for wind turbulence effects. The ability to } \\
\text { include turbulent wind effects is perhaps the biggest change in the code since the release of FLAP version 2.01 in 1988. This } \\
\text { report is intended as a user's guide. It does not contain a theoretical discussion on equations of motion, assumptions, underlying } \\
\text { theory, etc. }\end{array}$} \\
\hline \multicolumn{4}{|c|}{$\begin{array}{l}\text { 17. Document Analysis } \\
\text { a. Descriptors } \\
\text { wind turbines; loads; structures; computer codes }\end{array}$} \\
\hline \multicolumn{4}{|l|}{$\begin{array}{l}\text { c. UC Categories } \\
261\end{array}$} \\
\hline \multirow{2}{*}{\multicolumn{2}{|c|}{$\begin{array}{l}\text { 18. Availability Statement } \\
\text { National Technical Information Service } \\
\text { U.S. Department of Commerce } \\
\text { 5285 Port Royal Road } \\
\text { Springfield, VA } 22161\end{array}$}} & & $\begin{array}{l}\text { 19. No. of Pages } \\
214\end{array}$ \\
\hline & & & $\begin{array}{l}\text { 20. Price } \\
\text { A10 }\end{array}$ \\
\hline
\end{tabular}

Form No. 0069E (6-30-87) 